


Bosquet, $\phi$.

Lies Crustacés Fossiles du Terrain Bretacé du Deimboury. 



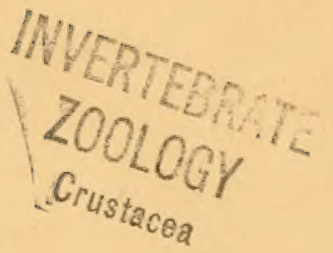

\section{LES CRUSTACÉS FOSSILES}

T E R R A I N C R E T A C E D U L I M B 0 U R G,

J. BOSQUET. 



\title{
CRUSTACES FOSSILES
}

\author{
DU \\ T E R R A I N C R E T A C É D U L I M B O U R G.
}

\section{A. CIRRIPEDIA.}

Fam. I. VERRUCIDAE DARwIN, 1851.

Gen. I. veremea Schumach. 1817.

Verruca Schumacher, 1817. Essai d'un nouveau Système. pag. 92, No. 3.

Creusia Lamarck, 1818. Histoire naturelle des animanx sans vertèbres. Tom. V, pp. 399, 400. Ochthosia Ranzani, 1820. Memorie di Storia naturale. pag. 28.

Clisia (SAvigny) Leach, 1825. Zoölog. Journ. Tom. II, pag. 210.

Ochthosia Buainvitue, 1825. Traité de Malacologie. pag. 597, pl. 85, fig. 4.

Deshayes, 1827. Dictionnaire classique d' Histoire naturelle. Tom. XII, pag. 52.

Verruca DARwin, 1851. A Monograph of the sub-class Cirripedia with figures of all the recent species (Ray Society). pag. 2.

Philippi, 1853. Handbuch der Conchyliologie und der Malocozoölogie. pp. 425, 426.

Caractères Génériques. - Coquille formée de six valves: deux scuta, deux terga, une carina et un rostrum. D'après les observations de Mr. DaRwin, ${ }^{1}$ ) ces valves sont symétriques dans le jeune âge et ne deviennent irrégulières qu’à une certaine époque du développement: la carina et le rostrum se rétrécissent alors d'un côté, tandis que de ce même côté le

1) Ce Naturaliste distingué a eu l'extrème obligeance de me communiquer les résultats de ses interessantes observations sur les caractères des parties dures et solides du Genre Verruca. 
scutum et le tergum se dévéloppent d'une manière extraordinaire et se joignent inséparablement, au moyen de dents ou saillies marginales articulaires, à la carina et au rostrum. Alors la coquille est formée de quatre valves: d'un scutum et d'un tergum fixes, d'un carina et d'un rostrum; et l'opercule ne se compose que de deux valves; c'est-à-dire, d'un scutum et d'un tergum mobiles.

Il est à remarquer que dans toutes les espéces du Genre Verruca, la coquille se fixe, tantôt par le côté droit, tantôt par le côté gauche: le hasard seul en décide. Si un individu s'attache par le côté droit, ce sont aussi les scutum et tergum du même côté qui deviennent irréguliers, en prenant un développement anormal. Dans le cas contraire, la même chose a lieu avec les scutum et tergum du côté opposé.

Il en résulte que parmi les espèces du Genre Verruca, il y a des individus dextres et des individus sénestres; et comme d'ailleurs la carina et le rostrum se ressemblent à tel point que dans les espéces vivantes on ne peut les distinguer à l' extérieur, qu’à leur position par rapport aux scuta et aux terga, il est naturel que dans les espèces fossiles il soit également impossible de faire cette distinction, à moins d'avoir à sa disposition des individus dont les valves sont restées réunies; ce qui n'a lieu que dans des cas excessivement rares.

Le Genre Verruca, dont on n’a eu jusqu'à présent que de bien fausses idées, a été rangé à tort dans la famille des Balanidae. Il forme à lui seul une famille particulière; à laquelle Mr. Darwin a donné le nom de Verrucidae dans son interessant et bien important ouvrage sur les Cirripèdes vivants.

La famille des Verrucidae, quoique intermédiaire entre les Lepadidae et les Balanidae, a de plus grands rapports avec les premiers qu' avec les derniers. Les Balanides n’ont commencé à se montrer à la surface du globe qu'à l'époque tertiaire.

1. verruga prisca Darwin in litteris; 1853 .

Pl. I, fig. $1-7$ et 7 .

Coquille à saillies articulaires peu nombreuses; à surface garnie de lames d'accroissement bien marquées et dépourvue de côtes longitudinales.

Scutum mobile (pl. I, fig. $2 a, b$ ), subtriangulaire, aplati; à bord occludent faiblement arqué, à sommet obtus et fortement incliné vers le tergum mobile; à bord tergo-latéral presque droit, mais offrant deux saillies articulaires, dont l'inférieure est beaucoup plus large que la supérieure. La partie articulaire déprimée est nettement séparée de la partie restante de la surface par une arête proéminente et aiguë, qui part du sommet et qui va se terminer sur l’angle baso-latéral; ce dernier est assez fortement saillant et terminé en pointe. Le bord basilaire 
est presque droit et forme avec le bord tergo-latéral, ainsi qu' avec la moitié inférieure du bord occludent, des angles droits. Toute la surface est couverte de lignes d'accroissement égales et équidistantes. Les bords internes sont faiblement proéminents; l'impression du muscle adducteur est orbiculaire et très-grande; la partie supérieure librement projectée est profondement concave et marginée à son côté occludent d'une crête étroite.

Le scutum mobile qui a été figuré, a 1,80 de millimètre de longueur.

Le scutum fixe (pl. I, fig. $1 a, b$ ) présente une forme tétragonale; son bord occludent est presque droit, le tergal légèrement excavé et le basilaire faiblement arqué (ce dernier est susceptible de varier: ces variations dépendent sans doute de la forme de la surface à laquelle le scutum a été attaché). La surface extérieure est divisée en deux régions distinctes, par une arête aiguë, qui part à une petite distance en dessous du sommet et qui se termine sur l'angle rostral. L'une de ces deux parties, la région externe, est subtriangulaire et couverte de lames d'accroissement imbriquées; l'autre région, la région articulaire, ne forme qu' une seule aire dans l'échantillon figuré; mais semble, d'après d'autres échantillons, (pour le reste beaucoup moins complets de ma collection), ètre divisée plus șouvent en deux aires articulaires. Les lignes d'accroissement qui recouvrent ces dernières, sont très-régulières et brusquement tournées en haut dans la partie la plus profonde ou les angles de ces aires.

Au côté interne, le scutum immobile (pl. I, fig. l b), présente comme celui des autres Verruca, une cloison triangulaire et assez profonde, formée par la lame transversale (incomplète dans l'échantillon figuré) contre laquelle est inséré le muscle adducteur. A côté de son angle externe supérieur, cette cloison est surmontée d'une crête très-proéminente, qui est marquée, comme toute la surface de la cloison, de lignes d'accroissement assez bien prononcées. Entre la partie supérieure du bord occludent et celle de la cloison, on voit ụne seconde crête plus courte, plus épaisse et marquée de lignes d'accroissement moins apparentes. L'impression du muscle adducteur est ovale-arrondie et assez profondement excavée. Le bord occludent interne est marginé d'une partie lisse, faiblement proéminente.

L'échantillon figuré a deux millimètres de hauteur.

Le tergum mobile (pl. I, fig. $3 a, b$ ) est aplati, assez large, subtétragone; son sommet obtus est fortement incliné vers le scutum mobile. Le bord carénal inférieur est droit et un peu plus court que le supérieur; ce dernier est arqué au sommet et droit dans ses deux tiers inférieurs; il forme avec le bord carénal inférieur un angle droit. La surface est divisée longitudinalement en deux parties inégales par une côte aplatie, faiblement arquée, obliquement tronquée et pointue à son extrémité inférieure. L'une de ces parties est plus large, subtriangulaire et couverte de nombreuses lignes d'accroissement régulières et parallèles au bord carénnl 
inférieur; l'autre est plus étroite, et partagée en deux aires articulaires: une aire inférieure plus large, et une aire supérieure plus étroite. Ces deux aires sont obliquement inclinées vers le centre de la valve et sont couvertes de lignes d'accroissement qui repondent aux bords articulaires.

Le tergum figuré de ma collection a 1,80 de millim. de longueur.

Le tergum fixe ( $\mathrm{pl}$. I, fig. 4,5 et 6 ) est irrégulier et très-différent du tergum mobile; cette différence cependant ne consiste essentiellement que dans un développement extraordinaire. Il est convexe et sa surface est partagée en trois régions: une région dorsale, couverte de lames d'accroissement transversales et assez irrigulières et deux régions articulaires. La région articulaire du côté scutal est toujours plus petite que celle du côté carénal. La première ne forme constamment qu'une seule aire, tandis que la seconde offre ordinairement deux, quelque fois même trois ou quatre aires articulaires. Toutes ces aires articulaires, dont la supérieure est constamment beaucoup plus large que les inférieures, sont couvertes de lignes d'accroissement très-régulières, parallèles aux bords et se relevant sous forme de lames imbriquées et assez brusquement courbées en haut, au fond des angles. La grande aire supérieure offre en oûtre sur sa partie médiane, un grand nombre d'autre lignes très-régulières et très-rapprochées les unes des autres; celles-ci coupent les premières à peu près à angle droit. L'angle de l'aire articulaire du côté scutal, présente un canal assez profond et lisse, à côté duquel les lignes d'accroissement sont courbées en haut comme dans les angles des aires articulaires du côté carénal. Au côté interne, le tergum fixe ne présente rien de particulier. Sa partie librement projetée est assez large et fortement recourbée en dehors. Le bord supérieur de la grande aire articulaire du côté carénal est marginé d'un bourrelet-saillant assez épais et marqué de lignes transversales d'accroissement.

Le tergum fixe fig. 3 , a 2 millim. de hauteur; celui qui est representé par la figure 5 a une hauteur de plus de 2,5 millim.

La carina? ${ }^{1}$ ) (pl. I, fig. 7 et $\gamma^{\prime} a, b$ ), est très-large; son bord basilaire est presque droit, tandis que le rostral offre des saillies ou dents articulaires assez profondes et ordinairement au nombre de cinq à six. La surface de la partie voûtée offre des lames d'accroissement imbriquées irrégulières, tandis que celle des aires articulaires est traversée par un grand nombre de lignes très-régulières, parallèles aux bords des découpures articulaires du bord rostral, brusquement recourbées au fond des angles et traversées obliquement par un grand nombre d'autres lignes,

1) Dans cette description je supposerai que cette valve soit une carina d'un individu attaché par le côté droit. La carina et le rostrum se ressemblent tellement, qu'il est absolument impossible de décider la question sans savoir si la valve provient d'un individu à côté droit fixe ou d'un individu à côté gauche fixe. 
plus fines et plus rapprochées les unes des autres. La région articulaire du côté tergal (fig. $\gamma^{\prime} b$ ) est subtriangulaire et est traversée par quelques côtes ou proéminences arrondies, qui partent du sommet et qui correspondent à des saillies articulaires peu sensibles du bord tergal. La surface intérieure n'offre rien de particulier; la partie librement projetée est large et creusée de sillons assez profonds et disposés en terrasses.

La carina? figurée de ma collection a presque 3 millim. de largueur, sur plus de 2 millim. de hauteur.

Rapports et Difrérences. - Suivant Mr. DARWIN cette espèce oftre des caractères intermédiaires entre ceux de la Verruca Stroëmia des mers qui baignent les parties septentrionales de l'Europe, et ceux de la Verr. laevigata des mers de l'Amérique du Sud. Elle a les plus grands rapports avec la première de ces deux espèces vivantes; mais s'en distingue très-nettement, en ce que la surface de sa coquille n'est pas costulée longitudinalement. Elle se rapproche de la Verruca laevigata en ce que la saillie ou côté articulaire inférieur du scutum mobile est plus large que la saillie articulaire supérieure.

Gisement et Localités. - Cette interessante Verruca se rencontre assez rarement dans le Duché de Limbourgo à St. Pierre, dans le calcaire grossier à Silex gris du Système Sénonien de Mr. Dunont, ainsi que dans les couches à Fissurirostra pectiniformis, qui forment dans cette même localité l'assise inférieure du Système Maestrichtien. Elle semble ètre extrèmement rare dans le calcaire grossier à Silex gris à Frère près de Tongres en Belgique. Mr. DarwiN possède dans sa collection un échantillon qui semble appartenir à la même espèce et dont les quatre valves de la coquille sont articulées ensemble. Cet échantillon, qui provient de la craie supérieure de Norwich en Angleterre, ne peut cependant être identifié avec certitude aux nôtres, parcequ'il est dépourvu dés valves de l'opercule, qui sont indispensables pour la distinction des espèces du Genre Verruca.

Fam. II. LEPADIDAE DARWiN, 1851.

Gen. 1. Mitelda Oken, 1815.

Mitella Oken, 1815. Lelirbuch der Naturgesclichte. pag. 362. (ante julium). Ramphidiona Schumaciter, 1817. Essai d'un nowveau Systène. pag. 97.

Pollicipes LeAch, 1817. Journal de Physique. Tom. LXXXV, Julius, 1817.

" " 1818. Apud Lamanck, Histoire naturelle des animaux sans vertèbres. Tom. V pp. $405-407$.

Polylepas Buarnville, 1824. Dictionnaire des sciences naturelles. 'I'om. XXXII, pag. 374. 
Polylepas Buainviule, 1825. Traité de Malacologie. pag. 524.

Capitulum (Kuein) Gray, 1825. Annals of Philosophy. Tom. X $2^{e}$ series, Augustus. Pollicipes DARwIn, 1851. A Monography of the fossil Lepadidae or pedunculated Cirripedes of Great Britain. pp. 47 et suivantes.

1851. A Monograph of the sub-class Cirripedia (Ray Society) pp. 293 et suivantes.

Caractères Cénériques. - Valves depuis dix-huit jusqu' à cent et plus. Latera du verticille inférieur nombreux; ayant les lignes d'accroissement dirigées en bas. Sub-rostrum existant constamment. Pédoncule squamifère ou spinifère.

Les valves des Mitella sont ou lisses, ou marquées de lignes d'accroissement bien distinctes; elles ont rarement des côtes longitudinales, ou des côtes transversales, correspondantes à chaque zone périodique d' accroissement. Les valves sont fréquemment fortes et solides, et leurs sommets sont projetés librement au dessus du Capitulum.

Comme ce sont les scuta qui offrent les caractères les plus constants et les plus importants pour la distinction des espèces fossiles du Genre Mitella, je les ai décrits (à l'exemple de Mr. DARWIN) avant toutes les autres valves.

Scuta en général trigones ou subtriangulaires, convexes et assez solides, présentant parfois un côté inférieur additionnel, qui resulte de la troncature de l'angle baso-latéral ou de l'angle rostral. Le bord tergo-latéral est ou excavé, ou droit, ou faiblement arqué. Le bord basilaire est ou droit, ou formé de deux lignes qui se rejoignent sous un angle large ou un peu irrégulier. L'angle, que forme le bord basilaire avec le bord occludent, varie beaucoup. Le bord occludent est en général faiblement arqué, il est quelquefois renforcé à l'extérieur par un rebord ou bourrelet. Dans diverses espèces une arête saillante part du sommet vers l'angle baso-latéral, ct dans un autre groupe d'espéces il y a une seconde arête, qui court vers un point à peu près médian du bord basilaire; dans ce dernier groupe les extrémités inférieures des deux arètes marquent sans donte l'étendue jusqu’à laquelle le rostrum et les latera supérieurs recouvrent le scutum. A l' intérieur, il y a constamment une excavation plus ou moins profonde, produite par le muscle adducteur des scuta. La partie supérieure de la valve est en général librement projetée et est marquée à l'intérieur de lignes d'accroissement; quelquefois il y a un sillon le long de la partie supérieure, soit du bord occludent, soit du bord tergal: dans ce dernier cas, le sillon sert à recevoir l'angle scuto-occludent, parfois pourvu d'une dent du tergum adjacent. Il n'y a que deux espèces, dans lesquelles le bord occludent offre à l'intérieur, à côté de l'angle rostral, une dent proéminente ou pendante. 
Terga: plus ou moins aplatis, rhomboïdaux, ou rarement pentagonaux, par suite de la troncature de l'angle basilaire.

Carina: plus ou moins fortement inclinée vers l'intérieur, rarement presque droite; elle s'élargit depuis le sommet jusqu'à la base plus rapidement que dans le Genre Scalpellum; en général une partie interne assez considérable se projette librement; cette partie supérieure est constamment bien moins concave que la partie inférieure. La valve est quelquefois tellement épaissie en cet endroit, que cette partie devient plate, et que parfois même elle offre une crête proéminente; le bord basilaire est obtusement pointu, ou arrondi, ou tronqué; les parietes sont en général plus ou moins sensiblement courbées en dedans, mais elles ne sont point séparées par une proéminence définie, ou par une arête, de la partie voûtée ou tectum; sur les parietes, les lignes d'accroissement sont transversales, ou en général seulement peu obliques. C'est par les caractères de la carina, que les Mitella peuvent être distinguées des Scalpellum, dans presque tous les cas.

Sub-carina: Il n'y a jusqu'ici qu' un très-petit nombre de Mitella fossiles, dont on connait la sub-carina; il est cependant très-probable qu'elle existe dans toutes, ou dans presque toutes les espèces. Mr. Darwin pense qu'elle pourrait bien manquer dans les Mitella unguis et glabra, parceque les latera carénaux de ces deux espèces sont très-grands. Il n'est pas impossible que ces deux espèces devront être érigées en un Genre, auquel la Mit. Bronni, (dont on ne comnaît jusqu’à présent que la carina) devra sans doute être rapportée également.

Rostrum et sub-rostrum: Quoique ces deux valves ne soient connues jusqu'à présent que dans un petit nombre d'espèces fossiles, on ne peut douter qu'elles n'existent dans presque toutes; le rostrum ressemble toujours à la carina, mais il est constamment plus court et proportiomnellement plus large; une plus grande portion de cette valve semble toujours avoir été projetée librement; ce qui est sans doute causé par la courbure plus abrupte de cette partie du capitulum. Je suis disposé à croire, que le sub-rostrum tout aussi bien que la sub-carina, n’a pas existé daus les Mit. glabra, unguis et Bronni.

Les latera supérieurs ne sont connus que dans les Mit. unguis, glabra, Darwiniana et concinna; dans les deux premières espèces, ils sont plats, triangulaires et très-grands; dans la Mit. Darwiniana ils sont triangulaires et très-petits, et dans la Mit. concinna, ils ressemblent beaucoup à ceux de la Mit. cornu copiae actuellement vivante. Les latera inférieurs de la Nit. concinna ont encore à peu près la même forme que ceux de la Mit. cornu copiae; dans les Mit. unguis et glabra, les sommets de ces petites valves ne sont pas projetés librement et ont un aspect différent de leurs homologues, dans toutes les espèces recentes; elles sont trigonales on obliquement tétragonales; de leur sommet part une côte ou une arête, 
qui se termine sur l'angle ou sur un point subanguleux, situé vers le tiers de la longueur totale du bord inférieur, qui est le plus souvent arqué. Je régarde comme un latus du verticille inféricur de la Mit. Darwiniana (n. sp.), unc valve triangulaire, très-épaisse et qui est un peu plus grande que le latus supérieur que j'ai cru devoir rapporter à cette espèce.

Le pédoncule est seulement connu dans la Mit. concinna; dans cette espèce, il est couvert de très-petites écailles quadrangulaires calcaires; je viens de découvrir des épines assez Iongues, qui me paraissent provenir du pédoncule de ma Mit. Darwiniana.

Le Genre Mitella, dont nous connaissons 6 espèces vivantes dans nos mers actuelles, est parmi les Cirripèdes pédonculés, celui qui s'est montré le premier à la surface du globe. Il est representé par une seule espèce dans l'Oölite inférieure; par deux dans l'Oölite moyenne, et par deux autres dans le Greensand inférieur, par une à deux dans le Chalk-marl; par trois dans la craie blanche sans silex; par six à sept dans la craie blanche avec silex; par huit dans le Système Maestrichtien ${ }^{1}$ ), et seulement par deux daus les divers Systèmes du terrain tertiaire.

Cette énumération montre que c'est dans les systèmes supérieurs du terrain crétacé, que le Genre Mitella atteint son maximum de dévéloppement spécifique..

Les trois espèces suivantes ont été trouvées dans les couches crétacées du Duclé de Limbourg.

1. mitella darwiniana nov. spec. 1853.

P1. 1 , fig. 8-16.

Scuta (pl. I, fig. $8 a, b, c)$ triangulaires, à sommet acuminé et fortement incliné vers les terga; à bord occludent faiblement arqué, formant avec le bord basilaire, qui est presque droit, un angle d'environ $35^{\circ}$; à bord tergo-latéral presque droit dans les jeunes individus, et plus ou moins fortement excavé dans les adultes; la 'moitié inférieure de ce bord forme avec le bord inférieur un angle droit ou presque droit. Du sommet de la valve part une arête, qui se dirige vers le bord inférieur et qui est presque parallèle au bord occludent. L'espace compris entre cette arête et le bord tergo-latéral, est couvert de sillons rayonnants. Ceite arête longitudinale se raccorde, par une pente très-rapide, à la partie déprimée qui se remarque le long du bord occludent. L'extrémité inférieure de cette partie déprimée, et par conséquent aussi les lignes d'accroissement qui la recouvrent, sont obliques et faiblement tournées en haut. Toute la surface est couverte de lignes d'accroissement, qui deviennent surtout très-apparentes, et qui se chan-

1) Quand on range dans ce Systime les couches crétacées supérieures de la Westphalie et celles de Schoonen en Suède. 
gent en plis, le long du bord tergo-latéral à l'extérieur, et le long du bord occludent à l'intérieur des Scuta. Au côté interne, le bord occludent est marginé d'un bourrêet très-large, dont la surface et partagée en trois portions bien distinctes: $1^{0}$. une supérieure toute couverte de tubercules arrondis et très-rapprochés; 20. une partie couverte de plis d'accroissement très-obliques et $3^{0}$. enfin, une partie lisse et unie, offrant à son côté externe une ligne longitudinale creuse et plus ou moins apparente. Cette dernière partie se termine inférieurement par une lame ou dent émoussée tétragonale, qu’ on peut déja apercevoir du côté externe de la valve; cette dent forme près de l'angle rostral une saillie très-remarquable (ce singulier caractère ne se retrouve que dans les Scuta de l'espèce suivante), il manque à toutes les autres. L’impression du muscle adducteur est située au centre, à l'extrémité supérieure de la partie concave de la valve; cette impression est très bien marquée et de forme ovalaire.

Le scutum figuré de ma collection a 19 millim. de longueur.

Terga (pl. I, fig. $9 a, b, c)$ à contour sensiblement pentagonal, assez larges, légèrement convexes; à sommet court, acuminé et faiblement incliné vers les scuta, à angle basilaire tronqué, extrêmement large; sa largeur étant plus grande que la longueur du bord occludent, celui-ci est extraordinairement court; le bord scutal en revanche est extraordinairement long, presque droit, faiblement excavé au milieu et arqué au sommet; il forme avec le bord occludent, ainsi qu' avec la partie tronquée basilaire, un angle de $110^{\circ}$; le bord carénal inférieur est droit et plus court que le supérieur; ce dernier est faiblement arqué dans sa moitié supérieure et droit dans sa moitié inférieure; cette dernière moitié forme avec le bord carénal inférieur un angle de $125^{\circ}$. La surface est couverte d'un grand nombre de lignes d'accroissement assez bien marquées; elle est ornée en oûtre de trois côtés longitudinales arquées et fortement déprimées: dont une médiane très-large, creusée de quelques lignes longitudinales obsolètes, (celle-ci repond à la troncature basilaire et s'élargit assez régulièrement depuis le sommet jusqu’à la base) et deux latérales très-étroites: dont l'une le long du côté carénal supérieur, et dont l'autre part du sommet et va se terminer un peu en dessous de la moitié de la hauteur du bord scutal. L'extrémité inférieưe de cette dernière repond constamment à une dent analogue à celle de l'angle rostral des scuta. Au côté interne, les deux tiers inférieurs de la surface sont lisses, tandis que le tiers supérieur est courert d'un assemblage de tubercules arrondis, devenant plus gros dans la partie centrale, et remplacés sur les bords par des stries proéminentes, terminées en pointe et rayonnantes. Celles-ci occupent les bords inférieur et scutal de l'assemblage. V'ers la moitié de la hauteur du bord scutal, la lame interne du test forme une saillie sémicirculaire subanguleuse, qu'on peut apercevoir du côté externe; et tout près de l'angle, formé par la réunion du bord carénal supérieur avec le carénal inférieur, on remarque encore une dent 
beaucoup plus petite et trop courte pour pouvoir être distinguée au côté externe. Les bords carénal supérieur et occludent, sont marginés d'une partic librement projetée, assez étroite et couverte de lignes d'accroissement.

Le tergum figuré se trouve dans ma collection; il a 16 millimètres de longueur.

La carina (pl.I, fig. $10 a-f$ ) est assez solide, allongée, fortement infléchie; elle s'élargit un peu plus rapidement dans sa moitié inférieure que dans sa moitié supérieure; son bord inférieur est protubérant; elle est fortement convexe, plus que sémi-cylindrique; elle est tellement épaissie dans le tiers supérieur de sa hauteur, qu'elle offre en cet endroit une section transversale à contour subtrigone. Elle est marginée, le long de chacun de ses deux bords latéraux extérieurs, d'une partie faiblement protubérante. Toute la surface est couverte de zones transversales d'accroissement, qui deviennent surtout très-apparentes sur les parties latérales renflées, et d'un grand nombre de sillons longitudinaux, qui disparaissent sur les parties latérales. $\mathrm{Au}$ côté interne, la partie lisse et unie, qui a immédiatement recouvert les tissus mous du corps de l'animal, est très-profondement concave et de forme subtriangulaire; la partie librement projetée est couverte de lignes et de plis d'accroissement bien marqués; elle offre au milieu une partie proéminente centrale qui s'élève sous forme de crète longitudinale.

La carina qui a été figurée, et qui se trouve dans ma collection, a 17 millimètres de longueur.

Rostrum (pl. I, fig. $11 a-f$ ) épais et solide, de forme triangulaire-subéquilatérale, ̀̀ sommet très-fortement infléchi (usé dans l'échantillon figuré), à bord inférieur largement tronqué et faiblement excavé au milieu, très-convexe, subsémicylindrique. Sa surface est couverte d'un grand nombre de plis d'accroissement bien-marqués, de quelques sillons longitudinaux, et sur la partie médiane, de deux côtes longitudiuales très-rapprochées. Ces côtes n'existent que sur le tiers inférieur du Rostrum; elles n’ont donc commencé à se former qu'à un certain age de l'animal. Plus de la moitié supérieure du côté interne a été projetée librement; la partie inférieure lisse et unie, qui a immédiatement recouvert les tissus mous du corps de l'animal, est subtriangulaire; la partie librement projetée est- presque tout-à-fait plate; cette dernière partie est creusée de plusieurs lignes transversales d'accroissement, mais n'offre aucune trace de la crête longitudinale médiane, qu’on observe sur la partie correspondante interne de la carina. Le rostrum offre au contraire en cet endroit un sillon longitudinal.

Le rostrum figuré de ma collection a $6 \frac{1}{2}$ millimètres de longueur.

Je possède une valve qui n'a que $3^{1} \frac{1}{2}$ millimètres de longueur et que j'ai cru inutile de figurer, parcequ'elle ne diffère du rostrum, que par ses dimensions plus petites, par son sommet non usé et par sa face interne concave dans toute sa longueur. Je ne doute presque pas que ce ne soit le sub-rostrum. 
Je pense que les deux petites valves representées sur la pl. I, fig. 13 et 14, sont des latera de la paire supérieure de la Mit. Darwiniana. Elles proviennent sans aucun doute de deux individus différents; elles sont assez épaisses; d'une forme triangulaire-subéquilatérale; leurs bords tergal et inférieur sont faiblement excavés, et le scutal est presque droit. Leur surface extérieure offre des lignes d'accroissement bien prononcées et quelques sillons longitudinaux obsolètes; les bords scutal et tergal sont marginés d'un bourrelet faiblement proéminent. Au côté interne, elles offrent toutes deux exactement les mêmes caractères que les latera supérieurs du Pollic. mitella, avec cette différence seulement, que leur partie librement projetée plus étroite est dirigée en dehors.

Le latus qui est representé par la fig. 13, a près de 2 millimètres de hauteur, sur $1 \frac{1}{2}$ de largeur; celui qui est representé par la figure 14, a deux millim. de hauteur, et sa largeur dépasse un peu la hauteur.

Je regarde comme un latus du verticille inférieur de l'espèce qui nous occupe, la petite valve de ma collection, qui a été figurée sur la planche I, fig. 12. Cette valve est très-épaisse et semble provenir d'un individu parfaitement adulte; sa forme est triangulaire-subéquilatérale; l'un de ses bords latéraux est faiblement; arqué et l'inférieur légèrement excavé au milieu. A l'extérieur elle est convexe et sa surface est couverte de lignes transversales d'accroissement bien prononcées. Le long de son bord arqué on remarque un sillon peu profond et parallèle au bord; la surface d'accroissement du côté interne est triangulaire et allongée transversalement; la partie supérieure librement projetée occupe plus que la moitié de la hauteur totale de la valve, et offre au milieu nne partic proéminente, aplatie en dessus.

Cette plaque n'a que 3 millimètres de largeur sur une hauteur à peu près égale.

Les valves spiniformes répresentées par les fig. $15 a, b, c$, paraissent provenir de l'espèce qui nous occupe. Je les regarde comme provenant du pédoncule, et Mr. D ai communiqué, est de mon avis. Ces épines, qui sont très-différentes de celles qui hérissent le pédoncule des Ibla, présentent des formes assez variables; elles sont plus ou moins allongées, plus ou moins larges à la base, et plus ou moins arquées dans toute leur longueur. Elles offrent constamment un grand nombre de stries transversales d'accroissement assez bien marquées et des étranglements très-forts, ordinairement au nombre de deux à trois. Immédiatement en dessous de chacun de ces étranglements, on observe, au côté externe, un anneau ou nodosité protubérante. La partie inférieure lisse et unie, c'est-à-dire la surface d'accroissement de ces plaques spiniformes est trigone, allongée transversalement, parfois ovalaire on arrondie; cette surface devient extrêmement petite dans certains échantillons (pl. I, fig. 16 b,) ce qui pourrait faire penser que quelques-unes de ces épines étaient caduques. 
Obserration. - Elles sont parfois d'une couleur rose-rougeâtre ou brunâtre. Cette couleur, qui devient plus apparente quand elles sont humectées, se retrouve dans les valves du capitulum.

IRapports et Diférences. - Cette Mitella est une des espèces les plus distinctes. C'est la seule connue jusqu’à présent, doint les térga, aussi bien que les scuta, offrent une dent marginale saillante, produite par la lame interne de ces plaques. Les terga de l'espèce suivante ne montrent aucune trace de cette dent (du moins dans le petit nombre d'échantillons que j'ai eu occasion de voir).

Gisement et Localités. - Cette belle espèce a été trouvée dans les couches à Fissurirostra pectiniformis du Système Maestrichtien à St. Pierre. Elle semble ne pas y être rare. Elle se retrouve en Belgique dans la même couche à Sichen et à Frère près Tongres.

2. initella valida Steenstr. spec. 1839.

Pl. II, fig. $1,2,3$.

Bec de Sèche (Loligo calmar) Faujas, 179s. Histoire naturelle de la montagne de St. Pierre, pag. 112, pl. XIX, fig. 1.

Bek van Loligo calmar (mar van eene onbekende soort) Traduction Hollandaise de Favjas par Pasteur, 1802. Natuurlijke Historie van den St. Pietersberg. pag. 150 , pl. XIX, fig. 1.

Pollicipes validus Stennstrup, 1839. Kroyer's Naturlistorisli Tidslirift. pl. V, fig. 28-32.

" gracilis Fr. Ad. Rowuer, 1841. Die Versteinerungen des Nord-deutschen Kreidegetirges. pag. 104, tab. XVI, fig. 14.

" validus DArwix, 1851. A Mlonograply of the fossil Lepadidae or Pedunculated Cirripedes of Great Britain. pag, 68, tab. IV, fig. $2 a-g$ (ALemoirs of the Palaeontograplical Saciety).

" gracilis Darwin, 1851. Ibidem. pag. 69, tab. IV, fig. $3 a, b$.

Valves remarquablement épaisses, massives et solides. Les seuta (pl. II, fig. $1 a, b, c$ ), sont subtrigones, allongés, $1 \frac{11}{2}$ a 2 fois aussi longs que larges, passablement convexes. 'loute leur partie supérieure est inclinée vers les terga. Dans les échantillons bien conservés, la surface de la moitié inférieure de la voûte dorsale, offre des stries longitudinales obsolétes et assez souvent disposées par paires. Le bord basilaire est formé de deux lignes, donnant naissance par leur réunion, à un angle très-ouvert. Le bord occludent est assez fortement arqué; il forme avec le bord basilaire, pris en entier, un angle d'environ $60^{\circ}$; le bord tergo-latéral est presque droit; il forme à peu près un angle droit avec la partie adjacente du bord basilaire. Le long 
du côté tergo-latéral on remarque une partie qui est couverte de stries d'accroissement tournées en haut; cette partie de la valve est étroite, n’ayant guère, dans sa portion la plus large, que la moitié de la largeur du dos aplati des scuta. Tout le bord occludent est renforcé à l'extérieur par un bourrelet saillant arrondi et fort; l'estrémité basilaire de ce rebord, et par conséquent aussi les lignes d'accroissement qui le recouvrent, sont obliques et faiblement tournées en haut. Du sommet de la valve partent deux arêtes: l'une de ces dernières court du sommet vers l'angle baso-latéral, et l'autre vers un point du bord basilaire, un peu plus rapproché de l'angle rostral, que de l'angle baso-latéral. Tout près de l'angle rostral, la lame interue de la valve est prolongée en bas en une dent émoussée assez forte, qu'on peut déja apercevoir du côté externe, et qui pend en dessous du bord basilaire comme dans l'espèce précédente. Le bord occludent interne est large; à peu près de la même largeur dans toute sa longueur; il est aplati et couvert en partie de nombreuses lignes d'accroissement. La partie couverte de stries est nettement séparée (daus les échantillons bien conservés) de la partie restante lisse et unie, par une ligne très-oblique. C'est cette dernière partic qui se termine inférieurement par la dent rostrale. Le creux, produit par'l'insertion du muscle adducteur, est très-profond. Entre l'impression musculaire et l'apex, on remarque, dans les échantillons adultes, une excavation assez profonde. Dans les jeunes individus et même dans les sub-adultes, cette excavation est moins profonde et beaucoup plus étroite. Dans l'échantillon figuré pl. II, fig. 1 b, je remarque, entre l'impression musculaire et le bord basilaire, un grand nombre de plis ou rides, interrompus, irréguliers et obliques.

Le scutum figuré de la collection de Mr. 'Thiereiss, a 24 millimètres de longueur.

Les terga (pl. II, fig. $2 a, b$ ) que j’ai trouvé dans la même couche crétacée et que je regarde comme appartenant à la même espèce, ont une forme ovale-subpentagonale; ils sont très-allongés et déprimés; leurs sommets sont fortement inclinés vers les scuta; leur bord occludent est très-court, tandis que le scutal est fort long et faiblement excavé en haut; le bord carénal supérieur est légèrement arqué, tandis que le carénal inférieur est droit; ces deux bords sont presque également longs et se raccordent sous un angle d'environ $100^{\circ}$. Toute la surfnce est couverte de lignes d'accroissement bien unarquées. Du sommet part une côte aplatie, assez fortement arquée, et s'élargissant lentement dans sou trajet. L'extrémité inféricure obliquement tronquée de cette côte, forme l'angle basilaire. Une proéminence ou côte aplatie beaucoup moins apparente, part du sommet à côté de la première, et est presque parallèle an bord occludent.

Le meillieur tergum que j'ai vu, se trouve dans la collection de Mr. Thierexs. Ce tergum est celui qui a été figuré; il a 19 millimètres de longueur.

La carina ( $\mathrm{pl} . \mathrm{II}$, fig. $3 a, b, c, d$, ) est assez large; elle s'élargit lentement et assez régulièrement du sommet jusqu'à la base; elle est extrêmement solide et fortement infléchie; 
sa partie supéricure librement projetée, égale plus de la moitié de la longueur. La section transversale de la valve est arquée, sub-géniculée à une petite distance de l'extrémité inférieure; dans la moitié de la longueur cette section est subdeltoïdale (et dans les échantillons un peu usés, elle est sémi-cylindrique, comme dans la figure $2 d$, de la planche IV de la Monographie de Mr. Darwin). La surface est couverte de zones d'accroissement inégales et de quelques sillons longitudinaux, dont les quatre latéraux seulement (deux de chaque côté) sont bien prononcés. Le bord basilaire est tronqué. Les angles latéraux ou talons sont évidemment saillants en dessous le long de chacun des deux bords latéraux extérieurs; ceux-ci deviemnent par là bien évidemment protubérants. La surface de la partie interne lisse, qui a immédiatement recouvert les tissus mous du corps de l'animal, est concave; cette partie est triangulaire et souvent même elle a la forme d'un triangle équilatéral; la portion interne librement projetée est tellement remplie de la substance calcaire, tellement épaissie, qu'elle devient plate; au centre de cette partie il s'élève parfois une crête longitudinale proéminente; cette crête est presque toujours moins apparente que dans l'espèce précédente.

La carina figurée de ma collection a 36 millimètres de longueur.

Obserration. - Le Pollicipes gracilis RoEver, me semble n'être qu'un jeune individu de la Mitella valida, comme Mr. Darwin l'avait déja pensé.

Eapports et Différences. - La Mitella valida a les plus grands rapports avec la Mit. dorsata Steexstr. ${ }^{1}$ ) du terrain crétacé (Système Maestrichtien) de Faxoë en Danemarck. Les scuta de cette dernière cependant (qui ont aussi le bord occludent renforcé par une côte) différent essentiellement de ceux de la Mit. valida, par leur angle baso-latéral largement tronqué, et surtout par les deux arêtes longitudinales qui divisent la surface en trois parties presque également larges. Les terga ont aussi une forme assez différente; leur grosse côte longitudinale est presque droite et beaucoup plus étroite, leurs sommets sont moins fortement courbés et leur bord occludent est bien plus long. Leur carina enfin est inoins convexe et beancoup moins recourbée.

Gisement et Localités. - Cette Mitella n’a été trouvée jusqu’à présent dans le Duché de Limbourg que dans les assises à Bryozoaires du Système Maestrichtien à St. Pierre, à Geulhem, à Bemelen et à Fauquemont. Dans cette dernière localité, elle a été observée aussi par Mr. Fr. Ad. Roener. D'après Mr. Stennstrup, elle se trouve à Ignaberga, à Balsberg,

1) Steenstrup, 1839. Kroyen's Naturhistorisk Tidskrift. B. II, pl. V, fig. 27 et 30.

Danwix, 1851. A MTonograply of the fossit Lepadidae or Pedunculated Cirripedes of Great Britain, pp. 69, 70 , pl. IV, fig. $4 a-f$. 
à Kjüge et à Morby (Schoonen) en Suède, ainsi qu’à Faxoë en Danemarck, dans des couches crétacées, qui appartiennent sans doute aussi au Système Maestrichtien de Mr. Dumont.

3. mitella glabra Fr. Am. Roemer, spec. 1841.

Pl. II, fig. 4-12.

Pollicipes glaber Fr. Ad. Roemer, 184.]. Die Versteinerungen des Nord-deutschen Kreidegebirges. pag. 104, tab. XVI, fig. 11.

" "

Reuss, 1845, 1846. Versteinerungen der Böhmischen Kreide-formation. Tab. V, fig. 48, 49; tab. XIII, fig. 86-91; tab. XXIV, fig. 11 et tab. XLII, fig. 17 .

"

(Roemer) Attr, 1849. Geognostisch-Palaeontologische Beschreibung der nächsten Umgebung von Lemberg. Pag. 30, tab. X, fig. 20.

Xiphidium maximum J. Sowerby (secundum Drxox), 1850. Geology and fossits of the Tertiary and Cretaceous Formations of Sussex. pag. 353, tab. XXVIII, fig. $6,7,5$.

Pollicipes glaber Darwin, 1851. A Monograply of the fossil Lepadidae or Pedunculated Cirripedes of Great Britain. pp. 61-64, pl. III, fig. $10 a-l$.

Scuta (pl. II, fig. $4 a, \delta$ ) passablement épais, assez larges, subtrigones; ì sommet pointu. Ils sont tellement convexes, que dans leur moitié supérieure ils ont à peu près la forme de la moitié d'un cône; leur bord basilaire n'est pas tout-ì-fait droit, il forme avec la partie inférieure $\mathrm{du}$ bord tergo-latéral, ainsi qu'avec le bord occludent, des angles moindres que des angles droits. La voûte dorsale de chacun des scuta offre deux arêtes longitudinales faibles; ces deux arêtes partent du sommet: l'une se dirige vers l'angle baso-latéral, et l'autre vers un point du bord basilaire un peu plus rapproché de l'angle rostral, que de l'angle baso-latéral; c'est justement le long de ces deux angles que la valve est le plus convexe. La surface est presque lisse; elle n'offre que des zones d'accroissement peu profondes, qui sont tournées en haut sur la partie tergo-latérale, et des lignes rayonnantes, extrêmement fines. Au côté interne, on aperçoit, immédiatement au dessus de l'impression du muscle adducteur, une excavation bien distincte, qui va abouter au sommet; le bord occludent interne est arrondi (dans les individus adultes) et conserve à peu près la même largeur jusqu'au sommet.

Le scutum figuré de ma collection n'a que 8 millimètres de longueur. J'ai trouvé plusieurs fragments qui indiquent des dimensions à peu près doubles.

Terga (pl. II , fig. $5 a, b, c$, ) rhomboïdaux, aplatis, présentant à peu près vers le tiers de 
leur longueur totale, au côté carénal, une arête longitudinale peu sailllante, presque droite, partant du sommet et allant se terminer à l'angle basilaire, qui est aigu; cette arète est abrupte à son côté carénal; de ce dernier côté, toute la surface de la valve est un peu déprimée. Le bord carénal supérieur se raccorde au bord occludent sous un angle de $60-70^{\circ}$; la longueur du bord occludent est à peu près égale à celle du bord scutal. Le bord carénal inférieur est constamment plus long que le supérieur. Le long du bord occludent une partie de la valve est faiblement protubérante; le long de cette partie on remarque une dépression longitudinale, au fond de laquelle s'élève parfois (dans quelques échantillons seulement) une côte plus ou moins apparente. Le bord scutal n'est pas tout-à-fait droit; la moitié inférieure (qui était sans doute en contact avec le latus supérieur) est faiblement saillante dans la plupart des échantillons.

Le tergum figuré de ma collection provient sous doute d'un individu parfaitement adulte. Il a 26 millimètres de longueur.

La carina (pl. II , fig. $6 a, b, c, d, e$ ) est subtriangulaire, et est terminée supérieurement en pointe; elle est pessablement forte et sa surface est lisse; elle est presque droite ou trèsfaiblement infléchie; sa section transversale présente un contour arqué; elle est subcarénée, et sa carène est ou simple, ou surmontée d'une côte faible et étroite, qui se termine au bord basilaire en une pointe émoussée plus ou moins apparente; les bords latéraux de la carina sont étroits, infléchis et présentent des lignes d'accroissement d'abord tournées en bas, et ensuite, sur le bord même, brusquement tournées en haut. La surface offre, dans quelques-uns de mes échantillons seulement, des lignes longitudinales creuses et très fines. Les deux angles baso-latéraux sont faiblement saillants; leur saillie est conforme à la direction des lignes d'accroissement indiquée ci-dessus. Au côté interne, la carina est profondement concave; le long des deux bords latéraux elle est canaliculée et la partie librement projetée n'occupe que le tiers supérieur de la longueur totale. Cette partie est concave et dépourvue de crête longitudinale.

La carina figurée de ma collection a 15 millimètres de longueur.

Le rostrum (pl. II, fig. $7 a-(b)$ a une forme triangulaire subéquilatérale; il est convexe, subcaréné, et sa carène est presque constamment surmontée d'une côte assez large, mais très peu saillante; le bord basilaire est très faiblement protubérant. La partie interne librement projetée, occupe à peu près un quart de la longueur totale; cette partie est remarquable (comme Mr. Darwis l'a déja fait observer) par une crête longitudinale, semblable à celle de la carina des Mit. valida, Darwiniana et carinata.

Le rostrum figuré de ma collection a 5 millimètres de longueur.

Le latus supérieur (pl. II, fig. 8 et fig. 9) est presque tout-à-fait plat, il a à peu près la forme d'un triangle équilatéral, mais avec les deux cotés supérieurs un peu inégaux et tous 
deux un peu plus longs que le bord basilaire; le sommet est tout-à-fait supérieur; sa longueur égale, suivant Mr. Darwn (qui a eu à sa disposition plusieurs valves provenant d'un même individu), un tiers de celle des terga; sa surface lisse, offre, le long du bord le plus long, une faible côte marginale, sur laquelle les lignes d'accroissement sont tres-sensiblement courbées en bas. A l'intérieur, les angles supérieurs sont coupés en biseau.

Le latus supérieur pl. II, fig. 9, provient sans doute d'un jeune individu, il n'a que $5 \frac{1}{2}$ millim. de longueur. Le fragment qui a été figuré pl. II, fig. 8, paraît provenir d'un vieil individu et a $6 \frac{1}{2}$ millim. de longueur.

Latera inférieurs: (pl. II, fig. 10,11, 11' et 12). Parmi les latera que j'ai trouvés et que je regarde comme appartenant au verticille inférieur, je remarque trois formes bien distinctes: $1^{0}$. fig. $12 a, b$, est un latus qui me semble provenir du côté de la carina; il est faiblement et assez régulièrement convexe; il a approximativement la forme d'un triangle allongé transversalement et avec un des angles coupé; ses deux côtés supérieurs sont un peu inégaux en longueur; le bord inférieur offre quatre échancrures peu profondes et inégales, qui ne sont pas reproduites sur les lignes d'accroissement. ${ }^{1}$ ) La surface est lisse; elle est obliquement traversée par une côte bien prononcée, sur laquelle les lignes d'accroissement peu marquées, sont légèrement courbées en bas, absolument comme sur la carène ou sur la côte de la carina, 20. fig. 10 , est un latus probablement du côté du rostrum: il a la forme d'un parallélogramme. La surface est nettement partagée en deux régions planes, en forme d'un triangle, dont l'un des côtés est plus court que les deux autres, par une côte plus on moins aplatie, à la surface de laquelle les stries d'accroissement sont fortement courbées en bas; l'angle auquel aboutit cette côte est émoussé; la surface des deux régions triangulaires est creusée de stries rayonnantes extrêmement fines: $3^{0}$. enfin, fig. 11 et $11^{\prime}$, sont des valves que je regarde comme des latera médians; $\left.{ }^{2}\right)$ ces latera ont à peu près la forme d'un triangle dont l'un des côtés est plus long que les deux autres; ce côté plus long est tantôt faiblement arqué dans toute sa longueur, tantôt il est sub-anguleux vers un tiers de sa longueur; l'un des deux autres côtés est faiblement arqué et le second légèrement excavé; la surface, qui est creusée de lignes rayonnantes plus ou moins nombreuses, est divisée par une arête arquée, plus ou moins aiguë, et non par une côte, en deux parties inégales.

1) Je ne remarque ces échancrures que dans un seul de mes échantillons. Il n'est pas impossible qu'elles ayent été produites pendant l'accroissement par le contact de quatre petites valves ou plutôt des squames adjacentes du pédoncule.

2) Si je compare le nombre des trois sortes de latera que j’ai trouvées dans une même couche crétacée, je remarque, que le nombre proportionnel des médians est à peu près double de celui des plaques que je regarde comme des latéra carénaux et quadruple de celles que je prends pour des latera du côté rostral. 
La plaque que je regarde comme un latus du coté carénal a près de 5 millimètres de largeur; celle que je prends pour un latus du côté rostral, n’a que 4 millimètres de largeur; l'un des latera médians a 5 millimètres et l'autre (qui est le plus grand que j’aie vu) a 7 millimètres de largeur.

Rapports et Différences. - Cette espèce a les plus grands rapports avec la Mit. unguis Sow. 1) du gault de Folkstone. Le petit nombre de caractères essentiels par lesquels cette dernière (dont on ne connait cependant pas encore les scuta) se distingue de la Mit. glabra, consistent seulement dans la grandeur rélative du latus supérieur, qui est moitié aussi long que le tergum et qui a le sommet situé sur la ligne longitudinale médiane et par les latera du verticille inférieur de forme différente et à angles latéraux bien moins aigus. D'après les figures et les descriptions données par Mr. DARwir, les valves de la Mit. unguis paraissent être en outre dépourvues des lignes longitudinales rayonnantes, qu'on observe presque constamment à la surface de celles de la Mit. Glabra.

Gisement et Localités. - Je viens de découvrir plusieurs valves de cette Mitella dans le calcaire grossier à silex gris du Système Sénonien et dans les couches à Fissurirostra pectiniformis du Système Maestrichtien à St. Pierre, dans le Duché du Limbourg. En Belgique, elle se rencontre dans les mêmes couches crétacées à Ciply près Mons, ̀̀ GrandLanaye, à Wonck, à Jandrain et à Folx-les-Caves. D'après Mr. DArwin, elle existe en Angleterre, dans la craie inférieure de Stoke-Ferry et de Norfleet, et dans la craie supérieure de Norwich, de Northfleet et de Gravesend, Kent; et selon Mr. Harris, dans le detritus de Charing, Kent. Suivant le Sénateur Hermavn Roemer, on la rencontre en Allemagne dans le pläner de Sarstedt près Hildesheim, d'après Mr. Fr. AD. Roemer, dans le kreidemergel, à LindenerBerge, près Hannovre. Selon le prof. Reuss, elle se rencontre abondamment dans le plänerkalk de Weisskirchlitz et n'est pas rare dans le plänerkalk inférieur de Schillinge près Bilin et dans le plänermergel de Luschitz et de Kystra en Bohème et enfin, d'après le Dr. ALti, elle est rare dańs le Kreidemergel de Nawaria en Gallicie. Mr. Darwin l'indique en outre, avec doute, dans le Système Maestrichtien de la Seandinavie.

Gen. II. scalpeluey Leach, 1817.

Lepadis spec. Linné, 1767. Systema Naturae.

1) Sowerby, 1837. Geological Transactions. $2^{\text {de }}$ series, vol. IV, tab. XI, fig. 5 et 5*.

DarwiN, 1851. A MLonograph of the fossil Lepadidae or pedunculated Cirripedes of Great Britain. pp. 64-69, tab. IV, fig. $1 a-l$. 
Scalpellum Leach, 1817. Journal de Physique. Tom. LXXXV. Juill.

Pollicipedis spec. Lamarck, 1818. Histoire naturelle des animaux sans vertèbres. Vol. V, pag. 407.

Polylepas Blatnville, 1824. Dictionnaire des Sciences Naturelles. Tom. XXXII, pag. 374.

Smillium (pars generis) Leach 1825. Zoölog. Journal. Vol. 2, Juill.

Calantica (pars generis) GraY, 1825. Annals of Phitosoply. Vol. X, (2d series) August.

Thaliella (pars generis) J. E. Grax, 1848. Proced. Zoölog. Society.

Xiphidium (pars generis) Drxon, 1850. Geology and fossits of the Tertiary and Cretaceous Formations of Sussex. pp. 222 and 353.

Scalpellum Сн. DARwin, 1851. A N Tonography of the fossil Lepadidae or pedunculated Cirripedes of Great Britain. pag. 13 et suivantes.

CH. DARwin, 1851. A Monograply of the sub-class Cimipedia with figures of all the recent species. pp. 215 et suivantes.

Caractères Génériques. - Valves au nombre de 12-15. Latera du verticille inférieur au nombre de quatre à six, à lignes d'accroissement le plus souvent convergentes. Subrostrum n'existant que très-rarement. Pédoncule squamifère ou très-rarement nu.

Jusqu'ici pas un seul échantillon fossile du genre scalpellum n’a été trouvé tout-à-fait parfait; mais, en jugeant par analogie, le capitulum était probablement formé de quatorze valves dans les Scalp. magnum, Darwinianum, Hagenowianum et radiatum; et de douze seulcment dans les autres espèces fossiles.

Les valves des Scalpellum sont ordinairement lisses, mais dans huit ou neuf espèces, elles sont marquées de côtes longitudinales; elles sont en général plus minces que celles des Mitella.

Carina: étroite, ne s'élargissant en général que lentement depuis le sommet jusqu'en bas; légèrement ou considérablement courbée vers l'intérieur; à parietes le plus souvent distinctement séparées par une arête, fréquemment surmontée d'une côte proéminente, du tectum ou toit. Ces parietes sont ou rapidement ou rectangulairement fléchies en dedans, et les lignes d'accroissement de leur surface sont très-obliques. Les intra-parietes, quand elles existent, donnent à la valve l'aspect d'être divisée en plusieurs parties, et semblent être intercalées, afin de remplir l'espace entre la partie supérieure de la carina et les terga; elles sont souvent séparées des vraies parietes par une côte, qui montre évidemment le contour normal de la valve. Dans plusieurs espèces ces intra-parietes se prolongent en haut au delà du sommet, qui devient par là subcentral; elles sont plates, et les stries d'accroissement de leur surface sont en énéral presque parallèles au bords internes de la valve. 
Scuta: le plus souvent très-faiblement convexes, quadrilatères; à bords tergal et latéral distinctement séparés par une arête. Le crochet des scuta est situé, dans la plupart des espèces fossiles, ¿̀ l'extrémité supérieure, mais daus quelques-unes, telles que les scalpellum magnum, radiatum, et Scalp? cretae, les lignes d'accroissement, au lieu de se terminer à l'angle qui sépare le bord latéral du bord tergal, sont prolongées vers le haut, de sorte que la valve est agrandie au dessus de l'umbo original. Les scuta des Scalpellum tuberculatum et Darwinianum présentent des caractères intermédiaires entre ceux de la plupart des autres espèces fossiles, pas exemple les Scalp. quadratum, fossula, etc. et ceux des Scalpellum magnum, radiatum et Scalp? cretae, ainsi que de la moitié du nombre des espèces vivantes actuellement connues. Le bord occludent des scuta est presque droit, ou faiblement arqué; ce bord, ainsi que le bord latéral, forme presque des angles droits avec le bord basilaire. A l'intérieur, la dépression pour le muscle adducteur est ordinairement assez manifeste; dans quelques espèces la valve est épaissie au dessus, dans d'autres au dessous de l'impression musculaire, ou parfois aussi entre cette impression et le bord occludent. Ce dernier bord est souvent aussi marginé de lignes d'accroissement; et la partie ainsi marquée s'élargit parfois tout-à-coup immédiatement au dessus de la fossette pour le muscle adducteur, ceci est causé par un faible changement de position du corps de l'animal pendant l'accroissement.

Terga: planes, de forme trigonale ou rhomboïdale; parfois tellement allongés et avec le bord carénal tellement excavé, qu'ils deviennent presque sémilunaires.

Le rostrum est encore inconnu dans la plupart des espèces fossiles, il est cependant à présumer qu'il aưa existé dans toutes. Je crois avoir découvert le rostrum de cinq, d'entre les sept espèces nouvelles que je décrirai ci-après.

Les latera supérieurs sont seulement connus dans six ou sept espèces fossiles; ils sont ovales-subtrigones, avec le crochet central dans le Scalpellum magnum; pentagonaux, dans les Scalpellum quadratum, pulchellum, fossula, gracile et pygmaeum; dans ces espèces un rebord interne se projette au delà des deux côtés supérieurs et indique déja une tendance à croître vers le haut; dans le Scalpellum radiatum ils sont trigones et offrent quelque analogie avec la plaque homologue du Scalpellum ornatum actuellement vivant. Les latera rostraux sont seulement connus dans les Scalpellum magnum, quadratum, pulchellum, gracile et pygmaeum; ils sont allongés et très-étroits. Les latera inframédians, qui sont encore inconnus dans la plupart des espèces fossiles, n'ont probablement existé que dans un petit nombre. On connaît les plaques du compartiment carénal des Scalpellum magnum, quadratum, fossula, maximum, pulchellum, gracile et pygmaeum. Dans les espèces fossiles et récentes les latera carénaux et rostraux s'accroissement principale- 
ment dans la direction de l'un vers l'autre, de sorte que leurs sommets sont situés tout près ou même en dehors des extrémités carénale et rostrale du capitulum.

Mr. Darwin n'a décrit les écailles du pédoncule que d'une seule espèce, à savoir celles dn Scalpellum quadratum; je décris ci-après des squames qne j’ai trouvées séparement; je les regarde comme provenant du pédoncule des Scalpellum gracile et pygmaeum (nov. spec.).

Le genre Scalpellum, dont on ne connaît que six espèces actuellement vivantes, nous offre un bien plus grand nombre de représentants fossiles. On en a signalé une espèce dans le greensand inférieur; une autre dans le gault; quatre dans le chalk-marl; une dans la craie blanche inférieure, et trois dans la craie blanche supérieure; trois dans le Système Maestrichtien, auxquelles viennent se joindre sept nouvelles, qui seront décrites ci-après; et seulement deux du terrain tertiaire. Mr. DARwin décrit en outre deux espèces; les Scalpellum angustum et tuberculatum du detritus de Charing. Comme ce terrain est formé d'un mélange de chalk-marl, de craie blanche inférieure et de craie blanche supérieure; on ne sait pas encore de laquelle de ces trois couches proviennent ces deux espèces.

On voit encore par cette énumération, que c'est dans les assises supérieures du terrain crétacé que le genre Scalpellum, tout aussi bien que le genre Mitella, a atteint son maximum de développement spécifique.

Huit espèces du genre Scalpellum ont été observées dans le terrain crétacé du Duché de Limbourg. Ce sont:

SECTION A. Espèces qui n'ont probablement eu que douze valves et dont la carina a son sommet tout-à-fait supérieur. Xiphidium DrxoN.

1. scalpellum maximum J. Sowerby, spec.

Pl. II, fig. 13-17.

Pollicipes maximus J. Sowerby. Mineral Conchyliog. tab. 606, fig. 4 et 6 .

Steenstrup, 1841. Kroyer's Naturhistorisk Tidskrift. B. II, pl. V, fig. 17, 18. medius Steenstrup, 1841. Ibidem. B. II, pl. V, fig. 13, 13*, 33.

sulcatus J. Sowerby. Mineral Conchyl. pl. 606, fig. 2.

maximus Fr. Ad. Roewer, 1841. Versteinerungen des Nord-deutschen Kreidegebirges. pag. 104, tab. XVI, fig. $9 a, \delta, c$.

" uncinatus? Fr. AD. Roeuer, 1841. Ibidem. pag. 103, tab. XVI, fig. $10 c{ }^{1}$ )

1) Je pense que la figure $10 c$, de la pl. XVI de Mr. Roemer représente un latus carénal du Scalp. maximum. 
Scalpellum maximum DARwiN, 1851. A MTonography of the fossil Lepadidae or pedunculated Cirripedes of Great Britain. pp. 20-35, pl. II, fig. 1-10.

VAR. $B$, INTERMEDIA BosQ. 1853. Carinae apice incurvato; intra-parietibus latioribus; superficie longitudinaliter obsolete sulcata; tecto, parietibus et intra-parietibus costis angustioribus inter se separatis.

Pollicipes ornatissimus J. MüLten, 1847. Monographie der Petrefakten der Aachener Kreide-formation. pag. 43 , tab. II, fig. $16 a, b$.

Remarques Générales. - Mes matériaux de cette espèce ne consistent qu'en un seul tergum, en quelques fragments d'un tergum d'une variété, et en un seul latus rostral, trouvés par moi dans le calcaire grossier) à silex gris; et en une magnifique carina, presque complète, qui a été trouvée par le Dr. J. MüutıR d'Aix-la-Chapelle, dans le kreidemergel de Vaels.

La carina presque complète (pl. II, fig. $13 a, b, c$ ) que le Dr. MüLler a bien voulu me prêter, constitue une nouvelle viriété du Scalpellum maximum à laquelle j’ai donné le nom de Varietas $B$ intermedia. Cette variété semble être intermédiaire entre les variétés typica et sulcata de Mr. Darwir. Je donne ci-joint une nouvelle figure (pl. II, fig. 14 $a, b, c)$ du fragment, décrit en 1847 par le Dr. Muluer, sous le nom de Pollicipes ornatissimus.

Elle est assez régulièrement arquée dans toute sa longueur et s’élargit graduellement depuis le sommet jusque vers son tiers inférieur. Le crochet n'est pas projeté librement dans le grand échantillon; mais dans le fragment de la même localité, fig. 14, une petite portion a du être librement projetée; le tectum est légèrement arqué, mais pas subcaréné; le bord basilaire se termine (d'après la disposition des lignes d'accroissement de la surface) en une pointe obtuse; les parietes sont faiblement concaves et à peu près de la même largeur que chacune des deux parties du tectum; elles sont séparées de ce dernier, ainsi que des intra-parietes, par des côtes arrondies, très-minces et très-étroites; les intra-parietes, qui sont plus larges que les parietes, sont aplaties et ne s'étendent pas jusqu' au bord basilaire de ces dernières. Les zones, produites par les accroissements successifs, sont très-bien marquées et sont accompagnées dans certains échiantillons (pl. II, fig. 14) de quelques côtes fort minces. Toute la surface est creusée en outre de plusieurs lignes longitudinales extrêmement fines et visibles seulement à l'aide d'un instrument fortement grossissant.

L'échantillon figuré pl. II, fig. I3, se trouve dans la collection du Dr. J. MüıLter d'Aix-la-Chapelle et a 24 millimètres de longueur; le fragment representé sur la même planche fig. 14 appartient au même Paléontologiste et n'a que $10 \frac{1}{2}$ millim. de longueur. 
Le tergum figuré pl. II, fig. $15 a, b$, me semble devoir être rapporté également au Scalpellum maximum; il a le plus de rapports avec la Var. I des terga qui ont été décrits par Mr. Darwin. ${ }^{1}$ ) Il offre cependant des différences, qui me font penser qu'il devra constituer encore une variété distincte. Il présente un contour subrhomboïdal, son bord occludent est plus long que le carénal supérieur; ses bords carénal inférieur et scutal sont presque également longs et se raccordent sous un angle de $35^{\circ}$; le bord scutal n'est pas tout-à-fait droit; vers la moitié de sa longueur il offre une saillie peu marquée, sans doute correspondante au sommet des latera supérieurs et produite par une côte déprimée, ou plutôt par un angle qui part du sommet, et à côté duquel les lignes d'accroissement n'offrent qu' une très-faible courbure. Sa surface, qui est creusée de lignes d'accroissement assez bien prononcées, est ornée dans son tiers médian de plusieurs lignes longitudinales creuses, très-faibles et qui vont se terminer sur le bord scutal.

Ce tergum se trouve dans ma collection; il provient du calcaire grossier à silex gris de St. Pierre, et a 21 millimètres de longueur.

Le tergum mutilé et figuré pl. II, fig. 16, semble appartenir à la variété II des terga décrits par Mr. Darwin. ${ }^{2}$ ) La figure représente l'échantillon, restauré au moyen de lignes ponctuées. D'après la disposition des lignes d'accroissement de la surface, ce tergum est trèsallongé, subtriangulaire, approchant de la forme d'un croissant, les lignes d'accroissement de la surface, quoique extrèmement fines, sont assez bien prononcées, et sont coupées par des lignes longitudinales creuses très-faibles et visibles seulement à l'aide d'une forte loupe; le bord carénal est faiblement excavé, presque droit, et ne peut pas être distingué en deux parties distinctes, comme dans la variété précédente; le sommet est fort allongé, étroit et presque en forme de corne; d'après d'autres fragments de ma collection, une partie assez considérable du sommet est projetée librement; le bord occludent est presque droit, et moins long que le bord scutal; ce dernier présente (d'après la disposition des lignes d'accroissement) vers le tiers supérieur de sa longueur, un angle qui marque le point auquel le sommet du latus supérieur et l'angle tergo-latéral du scutum adjacent, se rencontraient. La surface est divisée en trois parties distinctes par deux angles obtus, qui partent du sommet; l'un de ces angles correspond au point de convergence des stries d'accroissement et par conséquent à l'angle basilaire, et l'autre repond à l'angle, très-faiblement marqué, du bord scutal.

1) Je possède de cette variété quelques échantillons, provenant de la craie supérieure de Gehrden.

2) Darwin 1851. A Monograph of the fossil Lepadidae or pedunculated Cirripedes of Great Britain. pp. 30,31, ta'J. II, fig. 6. 
Le fragment figuré de ma collection a été trouvé dans le calcaire grossier à silex gris à St. Pierre et n'a que 7 millimètres de longueur; il semble avoir en environ 15 millimètres de longueur lorsqu'il était complet.

Le latus rostral, pl. II, fig. 17, qui me paraît provenir de la même espèce, est obscurement tétragonal; il est allongé, très-étroit, plus de deux fois aussi long que large; son bord basilaire est le plus long et faiblement arqué; plus que la moitié supérieure est assez fortement inclinée en dedans et le sommet est obtusement pointu. La surface est couverte d'un très-grand nombre de lignes d'accroissement extrêmement fines (fortement ressemblantes à celles du tergum fig. 16) et est creusée en oûtre de quelques sillons obsolètes, rayonnants du sommet vers les bords opposé et supérieur.

Le diamètre de mon échantillon unique est de 6 millimètres.

Rapports et Différences. - La carina du Scalpellum maximum a quelques rapports avec celle du Scalp. fossula. ${ }^{3}$ ) Elle s'en distingue cependant au premier abord, par ses dimensions, par sa largueur plus grande, par les côtes qui séparent les parietes du tectum beaucoup plus étroites, et enfin par son bord basilaire beaucoup plus pointu.

Gisement et Localités. - Cet interessant Scalpellum a été trouvé par le Dr. J. MüLter à Vaels près d'Aix-la-Chapelle, dans le Système Sénonien (Kreidemergel ou craie blanche sans silex). Les autres valves décrites ci-dessus ont été trouvées par moi dans la calcaire grossier à silex gris du même Système, à St. Pierre. Selon Mr. Fitcir, cette espèce se trouve en Angleterre dans la craie supérieure de Norwich; d’après Mr. J. Sowerby, dans celle de Northfleet, et suivant Mr. Harris à Charing, Kent. Mr. le Prof. Steenstrup l'indique dans le Grünsand de Köpinge en Suède, Mr. Fr. Ad. Roemer le cite dans le Kreidemergel de Gehrden ${ }^{2}$ ) et de Quedlinbourg en Westphalie; enfin, Mr. Crr. Darwin le mentionne de Ciply, près Mons, en Belgique.

2. SCALPELlum GRACile nov. spec. 1853.

Pl. III, fig. 1-9.

Remarques Générales. - De ce Scalpellum j’ai trouvé deux carinae entières et quelques fragments de la même valve; trois rostra, dont deux complets; trois ou quatre fragments de terga; quelques fragments de scuta; un seul latus supérieur, un peu mutilé; six latera

1) DARWin, 1851. A MFonograph of the Lepadidae or pedunculated Cirripedes of Great Britain. pp. 24, 25, pl. I, fig. 4.

`) D'où il m’a été communiqué par Mr. le Sénateur Hermann Roesier et par Mr. le Prof. Dr. Dencrmann de Hildesheim. 
carénaux; un seul latus rostral mutilé; et un assez grand nombre des squames, que je regarde comme provenant du pédoncule de la même espèce.

Carina (pl. III, fig. l $a, b, c$ ) moins forte et beaucoup moins solide que celle de l'espèce précédente; elle est médiocrement courbée; elle est assez étroite dans toute sa longueur et s'élargit graduellement depuis le sommet jusqu'à la base; elle est couverte de lignes d'accroissement nombreuses et assez bien prononcées; son sommet est pointu, faiblement infléchi dans l'échantillon figuré pl. III, fig. I; mais d'après quelques fragments et d'après l'échantillon figuré pl. III, fig. 2, le sommet est plus souvent notablement courbé en dedans. La partie centrale ou tectum est aplatie dans toute sa longueur et seulement très-peu convexe vers l'extrémité inférieure; elle est parfois creusée d'un sillon longitudinal médian superficiel; elle se termine inférieurement en une pointe émoussée et ses deux bords inféricurs se raccordent sous un angle de $65-70^{\circ}$. Le tectum est séparé des parietes, qui sont perpendiculairement infléchies, par des côtes très-peu proéminentes, proportionnellement plus larges en dessus, que celles de l'espèce précédente, et parfois presque entiérement effacées. Les parietes sont légèrement aplaties, très-étroites et très-faiblement concaves; elles sont parfois confondues avec les intraparietes (pl. III, fig. 2); mais elles en sont séparées ordinairement par une côte extrêmement étroite; les intra-parietes sont plus larges que les parietes, et leur surface est ornée de quelques sillons longitudinaux, qui coupent obliquement les lignes d'accroissement. D'après quelques fragments d'autres carinae de cette espèce, aucune partie du sommet n'a été librement projetée.

La carina de ma collection, pl. III, fig. 1, provient des assises inférieures du Système Maestrichtien de St. Pierre; elle a $25^{1} \frac{1}{2}$ millim. de longueur; celle qui est figurée pl. III, fig. 2, provient du calcaire grossier à silex gris de la même localité et a 32 millim. de longueur.

Le tergum incomplet pl. III, fig. 3, que j'ai cru devoir rapporter à la même espèce, est (d'après les lignes d'accroissement de la surface) très-allongé, rhomboïdal; son sommet est trèslong et sensiblement courbé vers la carina; les zones d'accroissement sont bien apparentes et quelques-unes se relevent sous forme de côtes; un sillon longitudinal peu profond court du sommet vers l'angle basilaire.

Le fragment figuré de ma collection n'a que 10 millimètres de longueur; ce tergum semble avoir eu 16-17 millimètres de longueur lorsqu'il était complet.

La valve, pl. III, fig. 4, que je regarde comme un latus supérieur ${ }^{1}$ ) de l'espèce qui nous occupe, présente un contour ovale-subpentagonal; son bord scutal est marginé d'un rebord

1) à cause de son analogie avec la valve homologue des Scalpellum quadratum, rutilum et rostratum. 
qui se projette en dessous, autour et au delà du sommet; le bord tergal est légèrement arqué et marginé d'un bourrelet faiblement proéminent et d'une partie légèrement inclinée en dehors; la surface de cette dernière partie et du bourrelet est couverte de lignes d'accroissement qui se dirigent obliquement en haut; entre le rebord marginal du côté scutal et la partie centrale de la valve on remarque un bourrelet aplati, très-nettement détaché de la partie restante de la surface; sur ce dernier, les lignes d'accroissement se courbent à angle droit vers le bord scutal; le bord inférieur est faiblement arqué; il est presque égal en longueur au bord tergal.

L'échantillon figuré a 6 millim. de longueur, il semble avoir eu à peu près $7^{1} \frac{1}{2}$ millim. de longueur, lorsqu'il était complet.

Les latera carénaux (pl. II, fig. 18 et pl. III, fig. 5 et 6 ) sont subtrigones; ils offrent, le long de leur côté concave, une partie assez étroite, à peu près rectangulairement infléchie et sur laquelle les lignes d'accroissement à peu près droites sont dirigées vers le sommet. Le bord opposé au crochet offre vers le milieu un angle d'oû part une arête, qui partage la surface on deux parties un peu inégales en largeur; l'une de ces deux parties est convexe, tandis que l'autre est déprimée. Les deux bords latéraux, c'est-à-dire le bord arqué et l'arête marginale du côté opposé, sont faiblement épaissis, quelquefois subtuberculeux (pl. III, fig. 5). Toute la surface est couverte de stries d'accroissement bien marquées et de plusieurs lignes longitudinales obsolètes. La partie interne librement projetée est large, assez profondement concave et marquée de lignes d'accroissement extrêmement fines.

Le latus carénal de ma collection pl. II, fig. 18, a 6 millimètres de longuneur; celui qui est representé sur la pl. III, fig. 5, n’a que 4 millimètres de longueur.

Les latera rostraux (pl. III, fig. 7) que j'ai cru devoir rapporter à cette espèce, sont obscurement tétragonaux; ils sont allongés très-étroits, plus de deux fois aussi longs que larges; ils s'étendraient sans doute parallèlement au bord inférieur des scuta comme ceux du Scalp. quadratum; ils s'élargissent graduellement depuis le sommet jusqu' au bord opposé, qui est arrondi; le bord inférieur est faiblement arqué. Pendant l'accroissement la valve s'est élargie le long du bord supérieur, et il s'est produit ainsi une partie très-large, obliquement tronquée au côté rostral et fortement inclinée. La surface offre un grand nombre de zones d'accroisscment bien marquées et deux côtes assez fortes, qui partent du sommet et qui vont se terminer le long $\mathrm{du}$ bord supérieur.

Je n'ai trouvé jusqu'ici qu'un seul latus rostral incomplet; il a 6 millimètres de longueur et semble avoir eu $7^{1 / 2}$ millimètres de longueur lorsqu'il était complet.

Je pense que la valve representée sur la pl. III, fig. $8 a, b, c$, est le rostrum de l'espèce qui nous occupe. Il a une forme trigone, un plutôt celle de la moitié d'une cône raccourci. 
Le bord irférieur est sensiblement excavé dans le tiers médian de sa longueur, et le sommet pointu est légérement infléchi. La surface de ce rostrum offre une partie médiane étroite, semblable au tectum de la carina, et deux parties latérales rapidement inclinées et representant les parietes de la carina. La surface du tectum est aplatie, très-faiblement arquée et les lignes d'accroissement qui recouvrent ce tectum sont parallèles à l'excavation du bord inférieur et par conséquent elles sont faiblement courbées vers le sommet. La surface de chacune des parietes est creusée de deux lignes rayonnantes vers le bord inférieur. Le rostrum, vu à l'intérieur, semble formé de deux ailes triangulaires, placées de manière à former à peu près un angle droit; plus que le tiers supérieur de la hauteur totale de la valve a été projeté librement.

L'échantillon figuré a un peu plus de 2 millimètres de hauteur, sur une largeur qui dépasse $2 \frac{1}{2}$ millimètres.

Les squames (pl. III, fig. $9 a, b$ ) qui paraissent provenir du pédoncule de l'espèce qui nous occupe, sont assez grandes en comparaison des valves; elles sont allongées transversalement et d'une forme tétragonale; leur bord inférieur est très-faiblement excavé et leurs extrémités sont obtusement pointues; leur surface, qui est couverte de zones d'accroissement analogues à celles des valves, offre dans son tiers médian supérieur une partie proéminente trigone; leur surface d'accroissement du côté interne est très-étroite, aplatie, lunulée, et la partie supérieure librement projetée occupe ordinairement plus du tiers supérieur de la hauteur totale.

Fapports et Dilrérences. - La carina a beaucoup de rapports avec celle de l'espèce précédente. Elle présente néanmoins de différences assez importantes: elle est d'abord beaucoup moins solide; elle s'élargit beaucoup plus lentement depuis le sommet jusqu'à la base; ses parietes sont bien plus rapidement infléchies, et son tectum, au lieu d'être assez fortement convexe, est aplati dans toute sa longueur, et seulement très-faiblement convexe vers l'extrémité inférieure. Les autres valves que j'ai trouvées dans la même couche crétacée, et que j’ai cru devoir rapporter à la même espèce, me semblent d'ailleurs présenter également des caractères assez importants.

Gisement et Localités. - Ce Scalpellum se rencontre assez rarement dans les couches à Fissurirostra pectiniformis du Système Maestrichtien, ainsi que dans le calcaire grossier à silex gris du Système Sénonien à St. Pierre. Elle se trouve en Belgique dans les mêmes couches crétacées à Grand-Lanaye, à Wonck, ̀̀ Frère près Tongres et à Folx-les-Caves.

3. sGalpellum Pygmaeum nov. spec, 1853.

Pl. III, fig. $10-17$.

Remarques Générales. - De ce Scalpellum j’ai trouvé plusieurs échantillons, tous plus 
ou moins mutilés de la carina; un seul tergum; deux plaques; que je regarde comme des latera supérieurs de jeunes individus, et un fragment du même latus provenant d'un individu adulte; deux échantillons incomplets du latus rostral; deux rostra à peu près complets; et plusieurs squames qui paraissent provenir du pedoncule de cette espèce.

Carina (pl. III, fig. $10 a-l$ ) très-étroite, presque droite dans ses deux tiers inférieurs; arquée dans son tiers supérieur; elle s'élargit lentement et assez régulièrement depuis le sommet jusqu'à la base. Cette dernière est obtusement pointue; ses deux bords se raccordent sous un angle de $80-85^{\circ}$, et dans les jeunes individus à angle droit, ou même à angle ouvert; le tectum est aplati et rejoint les parietes à angle droit; ces dernières sont légèrement concaves et nettement séparées du tectum et des intra-parietes, par des côtes arrondies, dont les deux supérieures ne sont nullement proéminentes, le long des deux bords lateraux du tectum; les bords inférieurs des parietes sont à peu près parallèles à ceux des intra-parietes. Les stries d'accroissement de la surface du tectum ne sont que très-peu marquées. A l'intérieur, la carina est faiblement concave dans sa moitié inférieure, et anguleuse ou canaliculée dans sa moitié supérieure. Rarement une très-faible portion du sommet se projette librement.

La carina figurée a 11 millimètres de longueur; elle peut avoir eu 13-14 millimètres de longueur lorsqu'elle était complète. D'après tous les fragments que j'en possède, je pense que ces dimensions sont à peu près les plus grandes qu'elle peut atteindre.

Le scutum incomplet, representé pl. III, fig. 11, me semble provenir de la même espèce. D'après les lignes d'accroissement de la surface, il offre un contour trapézoïdal; ses bords occludent et tergal sont droits, et se rejoignent sous un angle de $50^{\circ}$; son bord latéral est faiblement arqué; le basilaire, qui est droit, se raccorde au bord occludent sous un angle de $80^{\circ}$. Du sommet de la valve part une arête obtuse, qui se dirige vers l'angle baso-latéral, et qui partage la surface en deux parties distinctes; une partie convexe, presque lisse et marquée seulement de lignes d'accroissement très-faibles, et une partie faiblement déprimée, dont la surface est couverte de plis d'accroissement bien prononcés. Le bord tergal est marginé d'un bourrelet légèrement proéminent et le bord occludent d'une côte très-étroite. A l'intérieur, l'impression du muscle adducteur est placée au fond d'une partie assez profondement excavée; le long du bord occludent, un peu au dessus de l'impression musculaire des scuta, on remarque une deuxième excavation, obliquement inclinée vers le centre de la valve et de forme oblongue sémilunaire. Cette fossette semble avoir servie à loger le mâle ou les mâles, ou bien le mâle ou les mâles complémentaires.

L'échantillon mutilé qui a été figuré, a 6 millimètres de longueur; il peut avoir eu environ 9 millimètres de longueur lorsqu'il était complet. 
Le tergum representé pl. III, fig. 12, me paraît provenir de la même espèce; il est aplati, subrhomboïdal, ou irrégulièrement tétragonal; sa moitié inférieure est très-allongée et son angle basilaire est pointu. Le long du bord occludent on remarque une dépression longitudinale assez large, mais peu profonde, qui se termine un peu au dessus de la moitié de la longueur du bord scutal.

Ce tergum a $13^{1} \frac{1}{2}$ millimètres de longueur.

La plaque representée sur la pl. III, fig. 13, me semble être un latus supérieur d'un jeune individu de cette espèce. Elle est aplatie, allongée-subpentagonale; son bord scutal est faiblement concave, ses bords tergal et inférieur sont légèrement arqués. Le long du bord scutal ${ }^{1}$ ) on remarque deux bourrelets étroits, séparés par un canal assez profond et à la surface desquels les lignes d'accroissement sont brusquement recourbées vers le sommet; le long du bord tergal or aperçoit une partie faiblement inclinée en dehors, assez large et marginée de deux bourrelets arrondis, à la surface desquels les lignes d'accroissement sont faiblement courbées en haut. La partie centrale de la surface offre, outre les stries d'accroissement assez bien marquées, quelques lignes longitudinales obsolètes.

Le latus supérieur figuré de ma collection n'a que trois millimètres de longueur.

La plaque mutilée et representée pl. III, fig. 14, est un latus rostral que je crois devoir rapporter à l'espèce qui nous occupe. Cette plaque est très-allongée, presque trois fois aussi longue que large, son bord rostral est obliquement tronqué; le scutal est assez profondement excavé et l'inférieur un peu tortueux. La surface, qui est couverte de lignes d'accroissement assez bien marquées, offre une seule côte proéminente arquée, qui part du sommet et qui se dirige vers le bord opposé. Au côté interne, une partie assez notable du sommet a été projetée librement; cette partie offre une arête ou crête centrale bien prononcée.

L'échantillon a $2 \frac{1}{2}$ millimètres de longueur; il peut avoir eu $3 \frac{1}{2}$ millimètres de longueur lorqu'il était complet.

Je pense que le rostrum figuré pl. III, fig. 15, provient de l'espèce qui nous occupe. Il diffère essentiellement de celui que j’ai rapporté à l'espèce précédente, $1^{0}$. par ses dimensions un peu plus petites et par sa largeur moindre; 20. par son tectum un peu plus étroit et subcaréné; 30. par ses parietes bien plus rapidement infléchies et depourvues de sillons longitudinaux; et $44^{0}$. enfin, par la partie interne librement projetée plus large et égale à la moitié de la hauteur totale de la valve.

1) Le bord tergal est marginé dans les individus adultes (d'après un fragment de ma collection trop mutilé pour être figuré) d'un rebord très-étroit et projété en dessous, autour et au delà du sommet. 
Le rostrum figuré n’a que 2 millimètres de hanteur sur $2^{\frac{1}{10}}$ de millimètres de largeur. Les écailles provenant du pédoncule et figurées sur la pl. III, fig. 16 et 17, paraissent appartenir à cette même espèce. Elles sont allongées transversalement en forme de croissant; leur bord inférieur est faiblement excavé et les extrémités sont terminées en une pointe plus ou moins aiguë. La surface est presque lisse et n'offre que des lignes d'accroissement peu apparentes, et dans l'un (fig. 17), que j'ai rapporté avec beaucoup plus de doûte à la même espèce, I) la surface est creusée d'un grand nombre de sillons peu profonds et rayonnants du bord supérieur vers l'inférieur. La surface des deux plaques, offre immédiatement en dessous du sommet une partie triangulaire faiblement proéminente. Au côté interne, la surface d'accroissement est très-saillante et la partie librement projetée occupe plus que la moitié de la hauteur totale.

La plaque representée par la fig. 16 a 3 millimètres de largeur; celle qui est representée par la figure 17 n'a qu'un peu plus de $2^{1 \frac{1}{2}}$ millimètres de largeur.

Rapports et Difrérences. - La carina de cette espèce se rapproche de celle du Scalp. fossula par sa forme, par les côtes qui séparent les parietes du tectum et des intra, parietes, et surtout par son bord basilaire obtusement pointu. Elle s'en distingue cependant bien nettement ${ }^{2}$ ) par ses dimensions plus petites, par les côtes qui separent les parietes du tectum nullement proéminentes le long ides arêtes marginales supérieures et surtout par son tectum tout-à-fait plat et non subcaréné comme celui du Scalp. fossula.

Gisement et Localités. - Cette petite espèce se trouve dans les couches à Fissurirostra pectiniformis du Systène Maestrichtien à St. Pierre dans le Duché de Limbourg. On la rencontre en Belgique dans les mêmes couches à Wonck, à Grand-Lanaye, et à Folx-les-Caves.

4. SCAlPellum elongatum nov. spec, 1853.

P1. III, fig. $18,19,20$.

De cette espèce j'ai trouvé dans le Duché de Limbourg une seule carinà entière et un seul scutum mutilé. Comme dans la carina de notre Duché, les intraparietes n'ont pas été formées, 'j’ai cru devoir ajouter à mes dessins, des figures d'un échantillon incomplet de cette

1) Je commence à croire que cette écaille a plutôt appartenue à la variété du Scalp. maximum, qui est representée sur ma pl. II, fig. 16.

$\left.{ }^{2}\right)$ Mr. CIr. DaRwis de Farnborough ayant eu l'extrème obligeance de me prèter son unique échantillon du Scalp. fossula, je puis affirmer que l'espèce qui nous accupe ici est bien distincte de l'espèce Anglaise, avec laquelle je l'avais confondue au premier abord. 
même valve, mais montrant les intraparietes. Cet échantillon m’a été communiqué par Mr. le Prof. DE Konnck de Liège et provient du terrain crétacé de Ciply près Mons.

Carina (pl. III, fig. 18 et 19) très-allongée; assez régulièrement arquée; ne s'élargissant que lentement depuis le sommet jusqu'à la base; celle-ci est obtusement pointue, et ses deux hords latéraux se raccordent sous un angle de $60-65^{\circ}$; le tectum est légèrement convexe et obscurement subanguleux au milieu; il est séparé des parietes par une côte déprimée, peu saillante mais assez large; cette côte et les parietes sont très-rapidement infléchies; ces dernières ont à peu près la même largeur que les tecta, ou plutôt que chacune des deux parties du tectum; les intra-parietes (d'après l'échantillon figuré pl. III, fig. 19) sont beaucoup plus larges que les parietes; elles sont séparées de ces dernières par des côtes arrondies et fort étroites. Toute la surface extérieure est couverte de nombreuses lignes d'accroissement très bien marquées et réunies en faisceaux vers l'extrémité inférieure; la surface est creusée en ontre de nombreuses lignes longitudinales, que l'on n'aperçoit qu'à l'aide d'un instrument grossissant. Dans l'échantillon figuré pl. III, fig. 18, plus que le tiers supérieur a été projeté librement; cette libre projection me semble cependant n'être qu' accidentelle, et poưrait bien avoir été causée dans cet échantillon par le défaut d'intra-parietes; la partie librement projetée est couverte de lignes d'accroissement très-obliques; elle est creusée au milieu d'un sillon longitudinal assez profond; la partie inférieure semble devoir être profondement concave dans les échantillons ordinaires, c'est-à-dire non dépourvus d'intra-parietes.

La carina figurée pl. III, fig. 18, provenant du Système Hervien de Slenaken, a 37 millimètres de longueur.

Le scutum (pl. III, fig. 20) est allongé et d'une forme trapézoïdale; il est passablement convexe et, d'après la courbure de mon unique échantillon incomplet, son sommet doit être recourbé en dehors; le bord occludent est légèrement excavé et sa moitié inférieure se raccorde au bord basilaire sous un angle de $60^{\circ}$; le bord latéral est presque droit, légèrement arqué; l'angle basilaire aigu, correspond à un arête aiguë qui vient du sommet, et qui est séparée de la partie tergo-latérale déprimée par un sillon très-étroit, mais assez profond. Toute la surface extérieure, est couverte de lignes d'accroissement semblables à celles de la carina; elle est creusée en outre, comme cette dernière, de nombreuses lignes longitudinales inégales. Le côté interne de mon échantillon unique n'est pas à découvert.

L'échantillon mutilé a 12 millimètres de longueur.

IRapports et Différences. - La carina de ce Scalpellum se distingue aisement de celle des trois espèces précédentes, par sa forme très-élancée; par ses stries d'accroissement nombreuses, serrées et comme fasciculées; par ses lignes longitudinales très-rapprochées les unes 
des autres et surtout par la grosse côte aplatie et inclinée en dehors, qui sépare le tectum des parietes.

Gisement et Localités. - Jusqu' ici ce Scalpellum n’a été trouvé dans le Duché de Limbourg, que dans le psammite glauconifère du Système Hervien de Mr. Dunont, à Slenaken. En Belgique, il se trouve dans le Système Sénonien à Ciply près Mons.

5. SCAlpellum pulchellum nov. spec. 1853.

Pl. IV, fig. 1-6.

remarques Générales. - J'ai trouvé de ce Scalpellum un assez grand nombre de latera carénaux, dont plusieurs entiers; un très-petit nombre de latera rostraux; deux latera supérieurs et plusieurs fragments de ces latera; un seul rostrum; un tergum entier et un assez grand nombre de fragments de cette valve. Parmi mes fragments des scuta et de la carina, il n'y a pas un seul qui mérite d'être figuré. Le sommet des scuta est terminal, comme dans la valve homologue des espèces précédentes.

Les terga ( $\mathrm{pl}$. IV, fig. $1 a-c$ ) sont rhomboïlaux, aplatis, assez larges; leur angle basilaire est terminé en pointe. Les lignes d'accroissement de leur surface sont bien apparentes; elles convergent vers un sillon longitudinal peu profond, à peu près droit, qui part du sommet et qui se termine sur l'angle basilaire. Entre ce sillon et le bord occludent on remarque ordinairement $6-10$ côtes longitudinales minces, assez rapprochées les unes des autres et allant se terminer sur le bord scutal; entre le bord carénal et le sillon on n'observe constamment qu'une seule côte semblable. Les terga sont entièrement lisses à l'intérieur et la partie librement projetée est passablement large.

Le tergum figuré de ma collection a une longueur de 1 centimètre.

Le latus supérieur (pl. IV, fig. $2 a, b$ ) que j’ai cru devoir rapporter à l'espèce qui nous occupe, présente un contour irrégulièrement pentagonal; il est aplati, et son sommet faiblement proéminent est depassé par le rebord marginal assez large, qui entoure toute la partie supérieure de la valve. La surface est couverte de lignes d'accroissement bien marquées; et de plusieurs côtes légèrement arquées et s'épaississant assez sensiblement dans leur trajet d’une extrémité à l'autre. Parmi ces côtes, celles qui se terminent aux angles inférieurs sont plus grosses que les autres.

Le latus supérieur figuré à $6 \frac{1}{2}$ z millimètres de longueur.

Les latera carénaux (pl. III, fig. 21 et pl. IV, fig. 3 et 4), sont très-larges et d'une forme difficile à décrire; leur sommet est arrondi, proémiment, fortement incliné vers le centre de la valve et situé à peu près sur la ligne longitudinale médiane. Ils ont deux côtés assez 
fortement inclinés, et l'un de ces côtés est terminé par un angle plus ou moins saillant. Leur surface est couverte de côtes arquées, dont le nombre est assez variable. Parmi ces côtes, il y en a constamment deux qui sont plus grosses que les autres; dans quelques échantillons (qui semblent provenir de vieux individus) ces deux côtes sont aplaties et très-larges (pl. IV, fig. 4); elles forment à peu près les arêtes des deux parties inclinées.

Le latus carénal qui a été representé sur la pl. III, fig. 21 , à $3 \frac{1}{2}$ millimètres de longueur. Des deux autres, l'un a trois et l'autre cinq millimètres de longueur.

Les latera rostraux pl. III, fig. 22) sont très-étroits; à peu près $2^{1} \frac{1}{2}$ fois aussi longs que larges; ils sont assez fortement arqués et s'étendraient sans doute parallèlement au bord basilaire des scuta, comme ceux du Scalp. quadratum; ils s'élargissent graduellement depuis le sommet jusqu' au bord opposé, qui est arrondi subnnguleux; leur surface est ornée de quatre côtes longitudinales, arrondies et assez épaisses; le côté interne est arqué. Pendant l'accroissement la moitié rostrale de la valve devient épaisse, et en même temps cette dernière s'élargit le long du bord supérieur, en produisant ainsi un rebord large et incliné. Le sommet est pointu et le bord rostral est obliquement tronqué.

Le latus rostral figuré a près de 6 millimètres de longueur.

Le rostrum (pl. IV, fig. 5) est triangulaire, ou plutôt il a la forme de la moitié d'un cône; son bord basilaire est très-faiblemeut excavé et son sommet est pointu; sa surface est couverte de quelques côtes rayonnantes du sommet vers les bords; la médiane de ces côtes, qui est aplatie et beaucoup plus large que les autres, représente le tectum. Au côté interne, le rostrum est profondement concave et sa partie librement projetée est assez étroite.

L'échantillon figuré n'a que 2 millim. de hauteur sur une largeur de près de 3 millim.

rapports et Diférences. - Les terga de cette belle espèce ont quelques rapports avec ceux des Scalp. arcuatum ${ }^{1}$ ) DARwin du Gault de Folkstone en Angleterre, et Solidulum ${ }^{2}$ ) Steenstrup de Kjüge en Suède. Ils se distinguent des valves homologues de ces deux espèces par leur forme rhomboïdale, par leur angle basilaire beaucoup plus pointu, et surtout par les côtes longitudinales de la surface beaucoup moins nombreuses.

Gisement et Localités. - Cette espèce semble être propre aux couches à Fissurirostra pectiniformis du Système Maestrichtien. Elle n’a été trouvée jusqu'ici qu’à St. Pierre.

1) Danwin, 1851. A Monograph of the fossil Lepadidae or Pedunculated Cimipedes of Great Britain, pp. 40-42, tab. I, fig. 7.

2) Steenstrup, 1839. KRoYer's Naturhistoris/c Tidskrift. pl. V, fig. 14 et $14 \%$ 
SECTION B. Valves au nombre de quatorze ou quinze; crochet de la carina subcentral. Scalpellum et Smillium, LEacir; Calanthica et Thaliella, Gray.

6. SCALPEllum DaRWinianum nov. spec. 1853.

PI. III, fig. 6-12.

Carina (pl. IV, fig. $6 a-e$ ) assez fortement infléchie, géniculée vers le milieu; à sommet central, situé exactement au dessus de la courbure et un peu plus éloigné de l'extrémité supérieure que de l'inférieure. Les deux branches forment un angle d'environ $115^{\circ}$. La valve a une forme linéaire ou plutôt elle ressemble à deux carènes linéaires-lancéolées et soudées par le sommet; chacune des deux branches a son tectum particulier. Les tecta sont très-étroits, lisses, aplatis; ils sont seulement couverts de zones d'accroissement transversales, faiblement arquées. Le tectum de la branche supérieure offre deux faibles côtes marginales. Les parietes sont confondues avec les intra-parietes, très-larges et très-rapidement infléchies; leur sưface est ornée de nombreuses côtes filiformes, extrèmement minces et rayonnantes. Ces côtes, qui deviennent plus nombreuses sur la partie antérieure, sont faiblement arquées vers la partie médiane et presque droites sur les parties restantes de la surface. A l'intérieur, la valve est assez profondement. concave; elle est un peu plus étroite sous le crochet que dans le reste de son étendue, absolument comme la valve homologue du Scalpellum magnum du Coralline crag de l'Angleterre.

La carina figurée de ma collection a 17 millimètres de longueur.

Les terga (pl. IV, fig. $8 a, b, c$ ) sont allongés; leur sommet est pointu et fortement incliné vers la carina, et leur angle basilaire est obliquement tronqué; le bord occludent est arqué et le scutal est partagé par un angle en deux parties distinctes: une supérieure plus longue et légèrement sinueuse et une inférieure presque droite et un peu plus courte. C'est sans doute cette dernière partie du bord, qui a été en contact avec le bord du latus supérieur. Le bord carénal est droit; il offre seulement vers le tiers supérieur de sa longueur une proéminence bien distincte, qui marque sans doute la limite jusqu'à laquelle s'étend la carina. Ce dernier bord se raccorde à la partie inférieure du bord scutal sous un angle de $40^{\circ}$. La surface est elégamment ornée de $10-14$ côtes filiformes, extrêmement minces et de deux autres beancoup plus grosses. Toutes ces côtes sont croisées par des zones d'accroissement bien marquées et disparaissent sur l'angle médian formé par la convergence de ces dernières. Les deux grosses côtes sont à peu près parallèles au bord occludent; elle ne s'épaississent que lentement très-regulièrement dans leur trajet, et se terminent sur la partie supérieure du bord scutal. Le long du bord occludent, on remarque une partie déprimée assez large, obliquement tronquée ì son extrémité inférieure et parfois marginée (comme dans l'échantillon figuré) d'un bourrelet 
saillant. A la surface de cette partie déprimée et le long du bord carénal supérieur, qui est constamment infléchi, les zones d'accroissement, plus serrées que sur la partie restante de la surface, sont courbées en dehors. Au côté interne, les terga offrent, le long de tout le bord occludent, un espace couvert de tubercules arrondis. Ces tubercules deviennent plus nombreux vers le sommet. La partie interne librement projetée est faiblement concave et de forme sémilunaire.

Le tergum figuré de ma collection a $12 \frac{1}{2}$ millimètres de longueur.

Les scuta (pl. IV, fig. $7 a, b, c$ ) que je regarde comme provenant de la même espèce, ont le sommet placé sur le bord occludent, vers le quart supérieur de la longueur, en dessous de l'extrémité supérieure. Ils sont passablement convexes, irrégulièrement tétragones. Les bords se composent: d'un bord latéral, qui est un peu plus long que le basilaire et qui rejoint ce demier sous un angle de près de $100^{\circ}$; et d'un bord occludent supérieur et inférieur qui se raccordent sous un angle de 50-55\%. La partie supérieure du bord occludent est très-courte et obliquement tronquée au sommet. Cette partie correspond ici homologiquement au bord tergal de la plupart des autres espèces et à la partie supérieure presque droite des Scalpellum magnum et vulgare. Du crochet part une carène obtuse, qui va se terminer sur l'angle baso-lateral: cette carène partage la surface de la valve en deux parties triangulaires très-inégales: une supérieure, couverte de 5-6 côtes rayonnantes du sommet vers le bord latéral et une inférieure plus grande, dont la surface offre un canal bien distinct, au fond duquel les stries d'accroissement sont bien évidemment courbées en bas, et à côté de celui-ci, trois côteș filiformes droites, assez rapprochées les unes des autres, et ne s'épaississant que fort peu dans leur trajet. Ces côtes et ce canal partent de dessous le crochet et se terminent vers la partie médiane du bord basilaire. A l'intérieur, le scutum présente des caractères assez importants. L'impression ovalare du muscle adducteur est située à peu près sur la ligne longitudinale médiane, au fond d'une partie déprimée; cntre cette demière et le bord occludent les parties proéminentes sont convertes de tubercules arrondis épars ou disposés par rangées longitudinales. Ces tubercules sont semblables à ceux des terga. Le long du bord occludent enfin, on remarque une partie marginale étroite et inclinée en dehors; et à côté du crochet on voit une partie concave, assez large et marquée de stries d'accroissement. Cette partie concave est analogue à celle que présentent les scuta du Scalp. vulgare au même endroit. Elle a sans doute eu la même destination, c'est-à-dire celle de loger le mâle ou les mâles, ou bien le mâle ou les mâles complémentaires.

Le scutum figuré de ma collection n'a que 7 millimètres de longueur. Je possède des fragments, qui moutrent que cette valve atteint des dimensions plus grandes.

Les plaques figurées sur la pl. IV, fig. 9,10 et 11, sont des latera qui me paraissent. provenir de l'espèce qui nous occupe. 
Celle qui est representée par la figure 9, et que Mr. Darwin (à qui je l'ai communiquée) croit être un latus supérieur, a une forme obscurement tétragonale; ses bords latéraux sont assez fortement excavés. La surface offre deux parties bien distinctes: une inférieure, presque en forme de triangle équilatéral, et une supérieure, projetée en dessous, autour et au delâ du sommet. La première de ces deux parties est couverte de lignes d'accroissement transversales et n'est ornée que de deux côtes; la partie supérieure projetée au delâ du sommet est couverte de stries d'accroissement cencentriques et présente trois arêtes, qui rayonnent du sommet vers les angles du bord.

Ce latus a $2^{1 / 3}$ de millimètre de longueur.

La singulière valve, figure 10, pourraît bien être un latus rostral. Il a une forme difficile à déterminer. Sa partie projetée au delà et autour du sommet est obliquement tétragonale; les deux bords supérieurs de cette partie se rejoignent sous un angle de $80^{\circ}$; sa surface, qui est couverte de lignes d'accroissement concentriques au sommet et parallèles aux bords, offre trois arêtes, dont l'une va se terminer sur l'angle supérieur et dont les deux autres aboutissent aux deux angles latéraux. La partie inférieure, couverte de lignes d'accroissement transversales, est triangulaire; elle est ornée de cinq côtes longitudinales: deux marginales et trois intermédiaires.

Cette plaque a $3 \frac{1}{2}$ millimètres de longueur.

La valve representée par la figure 11, me semble être un latus carénal. Son sommet est proémiment et situé vers le tiers supérieur de la longueur; sa partie inférieure, converte de S côtes rayonnantes; est obliquement triangulaire, tandis que la partie projetée au delà et autour du crochet est obtusement anguleuse et marquée de stries d'accroissement concentriques.

Ce latus n'a que $2^{1 / 2}$ millimètres de longueur.

Le rostrum (pl. IV, fig. 12a-f) que j'ai cru devoir être rapporté à l'espèce qui nous occupe, est ovale-lancéolé. Son sommet est plus ou moins pointu; sa partie supérieure est triangulaire et son extrémité inférieure est arrondie. Toutes les lignes d'accroissement de la surface sont parallèles aux bords latéraux de la partie inférieure. Le rostrum est caréné et sa carène est limitée par deux sillons marginaux; chacune des deux faces latérales est ornée de L- ( ) côtes minces, filiformes et exactement semblables à celles des parietes de la carina. Au côté interne, la valve est assez profondement concave, et est creusée dans toute sa longueur, d'un canal médian assez large, mais peu profond; sa partie supérieure librement projetée est triangulaire; elle est inclinée vers le sommet et occupe plus des deux tiers de la longueur totale de la valve.

Le rostrum figuré a 7 millimètres de longueur. D’après les échantillons presque tous plus ou moins incomplets que j'ai trouvés, il me semble que la longueur de 7 millimètres doit être ì peu près la plus grande taille de cette valve. 
IRapports et Différences. - Parmi les espèces crétacées connues cette espèce et les deux suivantes (la dernière par analogie) sont les seules qui aient le sommet de la carina non terminal. Parmi les espèces tertiaires, il n'y a que le Scalp. magnum du coralline crag de l'Angleterre, dont la carina nous offre un exemple de cette même conformation. Par l'ensemble des caractères de la plupart des valves, le Scalp. Darwinianum se rapproche presque autant du Scalp. rostratum, qui vît actuellement sur les côtes des Iles Philippines, que le Scalp. magnum se rapproche du Scalp. vulgare des mers de l'Europe.

Gisement et Localités. - Ce Scalpellum n'a été observé jusqu’à present que dans les couches à Fissurirostra pectiniformis du Système Maestrichtien et dans le calcaire grossier à silex gris du Système Sénonien, à St. Pierre dans le Duché de Limbourg. En Belgique, il se trouve dans le Système Maestrichtien à Sichen, et dans le Système Sénonien à Frère près Tongres. Les scutum et tergum de cette belle espèce étant très-fragiles, ne s'obtiennant que difficilement entiers.

7. SGALPELlUm hagenowianum nov, spec. 1853.

Pl. IV, fig. 1:3, 1.4, 15 et 16

Remarques Générales. - De cette espèce je n’ai trouvé jusqu'à présent que deux carinae incomplètes, un seul tergum entier et plusieurs fragments de terga; un latus qui semble y appartenir et un seul rostrum que j'ai cru devoir y rapporter également.

Carina (pl. IV, fig. $13 a-c$ ) faiblement infléchic; à crochet situé vers le sixième de la longueur totale en dessous de l'extrémité supérieure; à tectum aplati, lisse, nettement séparé des parietes et offrant le long de chacun de ses deux bords latéraux un sillon marginal peu profond. D'après les lignes d'accroissement de la surface, l'extrémité inférieure doit être beaucoup) plus large et moins pointue que la supérieure. La valve a une forme lancéolée; elle est sensiblement comprimée près de son extrémité supérieure, qui se termine en une pointe émoussće. Les parietes sont confondues avec les intra-parietes; elles sont étroites dans la moitié inférieure de la valve et leur plus grande largeur est immédiadement en dessous de la courbure; toute la partie antérieure plus large est ornée de sillons rayonnants, qui partent de dessous le crochet et de la partie antérieure du tectum. Le bord marginal de l'espace garni de sillons, repond sans doute au point de contact des terga. Au côté interne, la plus grande profondeur de la carina est à peu près vers le quart supérieur de la longueur.

Le fragment figuré n'a que 7 millimètres de longueur. La longueur totale de la valve, lorsqu'elle était complète, semble avoir été d'environ 1 centimètre.

Les terga (pl. IV, fig. $14 a, b, c$ ) ont une forme triangulaire-subsémilunaire; ils sont 
aplatis et d'une épaisseur médiocre. Leur bord carénal est droit, ou très-faiblement excavé. L'angle basilaire est obtusement pointu. La ligne longitudinale, formée par la convergence des yones d'accroissement de la surface, est parallèle an bord carénal. Le sommet est assez large, arrondi, ct non terminé en pointe, comme dans les terga de toutes les autres espèces connues du genre Sealpellum. Le bord scutal est ì peu près aussi long que le bord occludent; ces deux bords se raccordent sous un angle d'environ 120\%; le bord occludent est marginé d'une partic déprimée fort étroite, obliquement tronquée ì son extrémité inférieure, et nettement séparée de la partie restante de la surface par une arête aiguë. Du sommet part une seconde arête ou plutôt une crête qui va s'effacer près de la moitié de la longueur du bord scutal; cette crête sépare la surface en deux parties inégales: une partic étroite, dont la surface plane est ornée de $6-S$ côtes longitudinales déprimées, très-minces et le plus souvent très-peu ap. parentes, et une partic plus large, dont la surface concave n'offre que $4-5$ de ces petites côtes. A l'intéricur, les terga sont tout-ì-fait lisses; leur partie supérieure librement projetée est asscz large et concave.

Le tergum figuré se trouve dans ma collection. Il a $9 \frac{1}{2}$ 'millimètres de longueur.

Lá plaque figuréc pl. IV, fig. 15 , pourriit bien être un latus rostral de l'espèce qui nous occupe. Elle est allongée, et sa surface est divisée en deux parties bien distinctes: une inférieure allongée, obliquement triangulaire et marquée sculement de quelques lignes transversales d'accroisscment, et une supéricure, arrondie, obscurement angulense et projetée en dessous, autour et au delì du sommet; cette dernière est couverte de stries d'accroissement concentriques au crochet et parallèles aux bords.

Ce latus a $2^{1 / \frac{2}{2}}$ millimètres de hauteur, sur 1 millimètre de largeur.

Rostrum (pl. IV, fig. $16 a, b$ ) très petit, ayant à peu près la forme de la moitié d'un cône raccourci. Il offre au milien une partie saillante, qui représente le tectum de la carina et qui est séparée des deux partics latérales (parietes) par une pente abrupte. Le sommet est acuminé et assez fortement infléchi. Le bord inférieur est très-sensiblement excavé au milieu et les deux moitiés de ce bord sont arquées. Le tectum est caréné et sa surface est creusée de phusieurs lignes longitudinales, très-fines et très-rapprochées; à la surface de chacume des deux parties latérales, on remarque $4-5$ sillons rayomunts, dont deux plus profonds que les nutres; ces sillons ont beaucoup de ressemblance asce cenx de la partic antérieure des parietes de la carina. La portion interne librement projetée est concave, et sa largeur égale à peu près le tiers de la hanteur totale de la valve.

Le rostrum n'a que $1 \frac{11}{3}$ millimètre de hauteur, sur près de 2 millimètres de largeur.

Bapporis et Difrérences. - La carina de cette espèce se distingue nettement de celle 
de toutes les autres qui ont été décrites: elle se reconnait facilement à ses sillons rayonnants du sommet vers les bords supérieurs et latéraux des parictes. Les terga se distinguent de ceux des autres espèces, (outre les autres caractères de leur surface) par leur sommet obtusement arrondi et non pointu.

Gisement et Localités. - Cette interessante espèce semble être assez rare. Elle n'a été trouvée jusqu'à présent, que dans les couches ì Fissurirostra pectiniformis, du Système Maestrichtien de la montagne de St. Pierre. J'ai rencontré un fragment d'un tergum du même Scalpellum, dans la même couche crétacée de Wonck en Belgique.

8. SGALPELLUM RADIATUM nov. spec. 1853.

Pl. IV, fig. 17-18.

Remarques Générales. - De cette intéressante espèce je n’ai trouvé jusqu'ici qu'un scutum un peu mutilé, qu'un seul latus supérieur, et que des fragments d'une autre valve, qui semble être un latus carénal. Les parties trouvées de cette dernière valve sont trop incomplètes pour mériter d'être figurées.

Scuta (pl. IV, fig. $17 a, b, c$ ) assez épais, fortement convexes et présentant un contour irrégulièrement tétragonal. Leur bord occludent est droit; il est excavé immédiatement au dessus du crochet, et se raccorde au bord basilnire (d'après la disposition des lignes d'accroissement et d'après un fragment de ma collection) sous un angle de $45^{\circ}$; lo bord tergal ou tergo-latéral est séparé par un angle en deux parties distinctes: une supérieure courte, et une inférieure beaucoup plus longue et faiblement arquée; cette dernière partie rejoint le bord basilaue sous un angle d'environ $115^{\circ}$, tandis que la partie supérieure plus courte se raccorde is la partie supérieure du bord occludent, sous un angle un peu plus grand qu'un angle droit. Le crochet est situé en dessous de la moitié de la longueur du bord occludent, et les lignes d'accroissement de la surface montrent que la valve s'est agrandie au moins aussi rapidement vers le haut que vers le bas. Du crochet part un canal assez profond, qui va se terminer sur le bord basilaire à une petite distance de l'angle rostral; au fond de ce canal les stries d'accroissement sont très-sensiblement courbées en bas. 'Toute la partie restante de la surface est couverte d'un grand nombre de côtes rayonnantes de l'umbo vers les bords. Ces côtes, qui s'élargissent assez sensiblement dans leur trajet et qui sont séparées par des interstices assez profondement concaves, sont plus étroites et plus rapprochées les unes des autres sur la moitié inférieure de le valve. A l'intérieur, l'impression pour le muscle adducteur des scuta est ovalaire et placée au fond d'une excavation assez profonde; et immédiatement en dessous de cette exeavation, la valve est assez fortement épaissie. Le long du bord occludent on remarque, à 
côté et au dessus de l'umbo, une partie allongée et concave, qui a probablement servi à loger le mâle ou les mâles, ou bien le mâle ou les mâles complémentaires.

L'échantillon figuré n'a que $7 \frac{1}{2}$ millimètres de longueur; il semble avoir eu une longueur de $5^{1 / 2}$ millimètres lorsqu'il était complet.

La valve representée sur la pl. IV, fig. $18 a, b$, appartient presque indubitablement à lá même espèce et semble être un latus supérieur. Elle est très-convexe; ses bords scutal et tergal sont droits et se rejoignent sous un angle nn peu plus grand qu'un angle droit; son sommet est obtus et le bord opposé (inférieur) est arqué aux deux extrémités. La surface, qui est garnie de huit côtes rayonnantes du sommet vers le bord arqué, offre un très-grand nombre de stries d'accroissement très-fines et quelques plis d'accroissement assez profonds et exactement semblables à ceux des scuta.

Le plus grand diamètre de l'échantillon est de $4 \frac{1}{2}$ millimètres.

Dbservation. - J'ai cru devoir regarder cette valve comme un latus supérieur, à cause de l'analogie que j'ai cru voir entre elle et la valve homologue du Scalp. ornatum ${ }^{1}$ ) GraY, spec, actucllement vivant sur la côte d'Algoa Bay, au Sud de l'Afrique. L'absence d'un sillon médian dans mon écbantillon fossile, ne me semble pas être d'une grande importance. Je pense cependant qu'on ne pourrait décider cette question d'une manière positive, qu'en comparant les carnctères de la face interne de ces valves. La surface du côté interne de mon échantillon fossile est malheureusement dans un très-mauvais état de conservation.

Mr. Pictet, dans son traité élémentaire cie Paléontologie ${ }^{2}$ ) a rapporté au genre 'Trigonellites (Aptychus H. von MEIJER), une valve du terrain crétacé de la Westphalie, antérieurement décrite par Mr. Fr. AD. Roener ${ }^{3}$ ) comme un scutum d'un Lepas, sous le nom d'Anatifera convexa. Cette valve, qui a à peu près la même forme que celle que je viens de décrire, pourrait bien êtro pareillement un latus supérieur d'une espèce du genre Scalpellum.

Itapports et Miférenees. - Le Scalpellum radiaŁum est la seule espèce connue jusqu’ì présent, dont l'umbo des scuta est aussi éloigné ou même plus éloigné de l'extrćmité supérieure que de l'inférieure.

Gisement et Localités. - Ce beau Scalpellum parait être plus rare que le précédent; il n'a été rencontré que dans la partie inférieure des couches à Fissurirostra pectiniformis du Système Maestrichtien entre St. Pierre et Petit-Lanaye.

1) Darwin, 1851. A MIonograph of the sub-class Cirripedia, with figures of all the recent species (Ray Society) pag. 244 , pl. VI, fig. 1 et $l^{\prime} a$.

2) Aptychus cretaceus Picter. Traité élémentaire de Paléontologie. Tome III, pag. 438.

3) Fr. AD. Roemen, 1840. Versteinerungen des Nord-deutschen Kreide-gebirges. pag. 103, tab. XVI, fig. 7. 


\section{B. ENTOMOSTRACA, von Munster.}

\section{LOPHYROPODA, LATREILLE.}

( O OSTRACOda, LatreiLie.

(Cyproïdes, Ostropodes, Milne Edwards; Ostracopodes, Eichwald; Cytherinidae, Burmeister).

Gen. I. CrTwerelda, Bosa. 1851.

(Sub-Genus, Jones, 1849).

Cytheres spec. von Munster, 1830. Jahrbuch für Mineralogie und Geologie von LEonhard und BRoNN, pp. 62 et suivantes.

Cytherina Liem and Lonsdate, 1838. Elements of Geology.

Cytherinae spec. Fr. Ad. Rommen, 1838. Jahrbuch fïr Inineralogie etc. von Lennard und Bromn. pp. 216, 517 .

spec. Fr. AD. Romien, 1840. Versteinerungen des Nord-deutschen Kreide-gebirges. pp. 104, 105.

spec. Reuss, 1845, 1846. Die Versteinerungen der Böhmischen Kreide-formation,

$1^{\text {ste }}$ Abtheilung, parg. 16, und $2^{\text {te }}$ Atheilung, pp. 104, 105.

Cytheres? spec. Connuer, 1846. Description des Entomostracés fossiles du Département de la Haute-

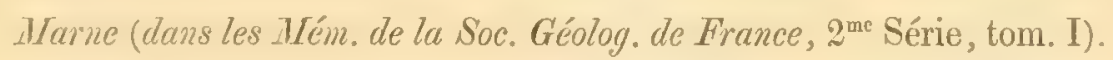

Cytherinae spec. Williamson, 1847. Transactions of the Manchester Literary and Philosophical Socicty. vol. VIII.

Cytheres spec. Bosquer 1847. Description des Entomostracés fossiles de la craie de Maestricht. pp. 6 -8.

spec. Bronn, 1848. Index Palaeontologicus. - Uebersicht der bis jetat bekannten fossilen Organismen. pp. 395, 396.

" ? spec. Connued, 1849. Description de nouveaux fossiles microscopiques du terrain crétacé infér. du Departement de la Haute-Narne (Mém. de la Soc. Géolog. de France, $2^{\text {me }}$ Série, tom. III, pp. 241-246).

Cythere (Sub-genus Cytherella Jones) 1849. A Monograph of the Entomostraca of the Cretaceous formation of England. pp. 28-33 (in the Memoirs of the Palaeontographical Society). 
Cytherinae spec. Reuss, 1849. Die fossilen Entomostraceen des Oesterreichischen tertiärbeckens, pag. 7 et suivantes. (Aus den Naturwissenschäftlichen Abhandlungen von Wilhem Hatdinger, III Band, $1^{\text {ste }}$ Abtheilung).

II spec. GeIniTz, 1850. Das Quadersandstein-gebirge oder Kreide-gebirge in Deutschland, Zweite Hälfte, Erste Lieferung, pag. 98.

Cytherella Bosquet, 1850. Description des Entomostracés fossiles des terrains tertiaires de la France et de la Belgique. pp. 9 et suivantes. (Académie Royale de Belgique. Mémoires des savants étrangers. tom. XXIV).

Cytherinae et Cypridinae spec. Reuss, 1851. Naturwissensch. Abhandl. von Wili. Haidinger. Vierter Band, $1^{\text {ste }}$ Abth. pag. 47-50.

Caractères Génériques. - Carapace formée de deux valves de consistence cornée ou corneo-crétacée, mobiles, inégales, réniformes, oblongues, ovales ou ellipsoïdales, et plus ou moins déprimées.

Ces valves sont le plus souvent lisses à l'extérieur, ou creusées de points, ou granuleuses, ou tuberculeuses; elles sont assez souvent ornées de bourrelets, et leur surface n'offre jamais des côtes concentriques ou des épines.

La valve droite des Cytherella est constamment plus grande que la valve gauche, ${ }^{1}$ ) et embrasse un tant soit peu toute la circonférence du bord de celle-ci, quand la carapace est fermée. Le bord interne de la valve droite, qui est toujours plus large que celui de la valve gauche, offre le long de son côté interne un sillon abaissé ou rabat assez profond. Sur la valve gauche, on observe la même chose en sens inverse, mais cependant avec cette différence, que la partie externe abaissée n'est sensible que le long des bords postérieur, supérieur et inférieur. La partie interne plus haute du bord de cette dernière valve, est aussi plus large que la partie abaissée externe, le long des bords supérieur et inférieur; tandis qu'elle est d'une largeur ì peu près égale à cette partie, le long du bord postérieur.

Sur cette même valve, la partie interne plus haute du bord est plus large que la partie interne abaissée (sillon) du bord de la grande valve, et ne peut par conséquent, lors de la r'éunion des deux valves, s'insérer dans ce sillon que partiellement, et seulement par son côté aigu ${ }^{2}$ )

1) Cette disposition des valves des Cytherella est l'inverse de celle de ces mêmes parties dans tous les autres genres de la famille des Ostracodes.

2) Voyez mon Mémoire sur les Entomostracés fossiles des terrains tertiaires de la France et de la Belgique. pl. I, fig. $1 b, c$. 
Les valves des Cytherella présentent à l'intérieur, entre le centre et le bord supérieur, un tubercule arrondi-oblong et dont la direction est constamment oblique à leur axe longitudinal. Ce petit tubercule, quoique assez nettement limité, est très-peu proéminent; il serait le plus souvent presque imperceptible et échapperait par conséquent facilement à l’observateur, si sa coloration blanchâtre et sa texture matte ne contribuaient pas à le faire distinguer de la partie restante de la paroi interne.

Ce petit tubercule interne répond à une très-petite fossette externe, peu marquée chez la plupart des espèces, mais devenant très-apparente chez quelques-unes, comme par exemple chez ma Cytherella hieroglyphica et chez la Cyth. Williamsoniana JoNes, qui présentent, au fond de cette fossette, une petite protubérance oblongue très-apparente

On connait actuellement onze à douze espèces se rapportant au genre Cytherella dans la formation crétacée, cinq à six dans la formation tertiaire (deux de ces dernières ont leurs identiques dans le terrain crétacé) et une espèce vivante, encore inédite, dans la Méditerranée. De sorte que jusqu'ici on ne peut signaler que quinze à seize espèces de ce genre.

1. Gythenella ovata Roemer. spec. 1840.

PI. VIII, fig. $1 u, b, c, d, e, f$.

Cytherina Lyeut and Lonsdale, 1848. Elements of Geology. pag. 55, fig. 19.

ovata Fr. AD. Roemer, 1840. Die Versteinerungen des Nord-deutschen Kreidegebirges. pag. 104, pl. XVI, fig. 21.

complanata Reuss, 1844. Geognostische Skizzen aus Bölmen. Band II, pag. $21 \%$ elongata Reuss, 1844. Ibidem. Band II, pag. 217.

ovata Reuss, 1845. Versteinerungen der Bölmischen Kreide-formation. $1^{\text {ste }}$ Abtheil.

pag. 16 , tab. V, fig. 35 .

complanata Reuss, 1845. Ibidem. pag. 16, tab. V, fig. 34 .

elongata Reuss, 1844. Ibidem. pag. 16, tab. V, fig. 36.

Cythere reniformis Bosquet, 1847. Description des Entomostracés fossiles de la craie de Naestricht. pp. $6,7, \mathrm{pl}$. I, fig. $a-f$.

Bosquet, 1847. Mémoires de la Société Royale des Sciences de Liége. Iom. IV, pp. 356,357 , pl. I, fig. $1 a-f$.

Cytherina laevis Wibliamson, 1847. Transactions of the Manchester Philosophical Society. pl. IV, fig. 80 ,

Cytherella ovata Jones, 1849. A NTonograph of the Entomostraca of the cretaceous formation of Eingland. pp. 28- 30, tab. VII, fig. 24 $a-i$. 
Cytherina ovata Reuss apud Geinitz, 1850. Das Quadersandstein-gebirge oder Kreide-gebirge in Deutschland. pag. 98, No. 5 .

" complanata Reuss apud Geinitz. Ibidem. pag. 98, N0. 2.

" elongata Reuss apud Geinitz. Ibidem. pag. 98. No. 8.

" ovata Reuss, 1851. Foraminif. und Entomostr. des Kreidemergels von Lemberg. (Naturw. Abhandl. von Wilfr. Haidinger. IV Band, ${ }^{\text {ste }}$ Abth. pag. 48 , tab. VI, fig. $2 a, b, c$.

Leopolitana Reuss 185̃1. Ibidem. pag. 48, tab. VI, fig. $3 a, b, c$.

Les valves des individus adultes de cette espèce sont ovales-oblongues ou sub-réniformes; leur surface est en géneral lisse et lưsante; elles sont arrondies aux deux extrémités; leur bord supérieur est arqué, ou bien il affecte la forme de la moitié d'une ellipse; le bord inférieur est presque droit, plus ou moins infléchi vers le milieu, d'oú vient que la carapace fermée acquiert assez souvent la forme d'une fève. La valve gauche est constamment plus étroite que la valve droite, et son bord dorsal est toujours moins arqué que celui de cette dernière. Les valves des jeuncs individus sont ovales, plus larges dans leur moitié antérieure que dans la postérieure. ${ }^{1}$ ) Mr. R. Joves en a trouvé de plus jeunes encore, qui offraient à la surface de petites épines. Parmi mes mombreux échantillons j'en remarque quelques-uns aussi, qui paraissent provenir d'individus subadultes, et qui présentent un contour ovale-allongé. Les bords supérieur et inférieur de ces derniers sont faiblement arquués; ils ont donc exactement la forme de la valve qui a été figurée dans le Mémoire de Mr. Jones, sur la pl. VII, fig. 24 l.

La carapace est plus ou moins convexe suivant l'âge. Dans les jeunes individus elle est plus déprinée, dans les adultes sa plus grande convexité est vers le tiers postérieur de la longneur. Elle offre une section transversale à contour ovalaire, et une section longitudinale à contour ovalc-allongé.

Quelques-uns de mes échantillons sont demi-transparents et d'une couleur rouge-purpurine. Cette couleur est, je pense, um reste de celle qu'ils avaient pendant la vie des animaux.

Dimensions. - Longueur 1 millim., hauteur 0,60 de millim. et épaisseur 0,45 de millim.

ouservation. - Ayant reçu de Mr. Reuss des échantillons de ses Cytherina complanata et clongata, je puis affirmer que ces deux espèces n’ont été établies que sur de légères variations de forme (sur des différences d'âge ou des caractères d'échantillon) de la Cytherella ovata de Mrr. Fr. AD. Roesier. - Si je n'eus pr en juger que d'après les figures qui ont

1) C'est sur un pareil échantillon jeune que Mr. FR. AD. RoEmer a établi l'espèce. 
été données par l'auteur de Bilin, je n'aurais bien certainement pas pu faire ce rapprochement.

Rapports et Différences. - La Cytherella compressa von Münster spec. ${ }^{1}$ ) des depôts tertiaires Miocène et Pliocène, est bien voisine de celle-ci. Il n’est cependant pas difficile de la distinguer au premier abord de l'espèce crétacée: ses valves sont constamment plus larges aux extrémités et le dos de ces valves est toujours le plus bombé dans le tiers postérieur de sa longueur, d'oú vient que la carapace fermée offie une section Inngitudinale à contour cunéiforme.

Gisement et Localités. - La Cytherella ovata est une des espèces les plus abondantes parmi les Entomostracés des couches crétacées. Je l’ai trouvée dans le Duché de Limbourg dans le Système Maestrichtien à St. Pierre, à Nedercanne, à Gronsveld, à Bemelen, à Geulhem, à Fauquemont, à Vieux-Fauquemont, à Kunraede, à Bulkomsdal et dans une conche qui paraît appartenir également au Système Naestrichtien à Schin-op-Geul; dans le Système Sénonien (calcaire grossier ì silex gris) entre St. Pierre et petit Lanaye, dans le même Système, craie blanche svec silex, au Nord c'e Wahlwiller près Wittem et dans la craie blanche à Slenaken, à Pesaken, ì Galoppe et entre Vaals et Gimenich, è dans une couche qui paraît dépendre du Système Hervien entre Benzenrathhof $e_{s}^{\ddagger}$ Keverberghof près Heerlen. Entre Vaals et Gimenich, à Pesaken, ì Slenaken et ì Simpelveid, jo l'ai trouvée également dans le psammite glauconifère (appartenant au Système Hervien de Mir. Dusiont). Elle se trouve en Belgique dans le Système Maestrichtien à Sichen, à Jandrain et à Folx-les-Caves; dans ce même Système et dans le calcaire grossier à silex gris (Sysíčme Śnnonien Duxont) à Ciply près Mons et dans la craie blanche (du même Système crétacé) à Jandrain et à Heure-le-Romain. D'après Mr. FR. AD. Roener eile se rencontre dans la craie marnense inférieure de Lemförde dans le Nord de l'Allemagne. Selon Mr. AuG. Exr. Reuss elle est abondante dans lo pläuerkalk de Kosstiť et de Kutschlin, rare dans le plänermergel de Priesen et d'Aannay en Bohème et assez abondante dans le Kreidemergel de Lemberg, en Gallicie; suivant Mr. Ropert Jones elle se trouve en Angleterre dans le greensand de Warminster, dans le gault de Folkstone et de Leacon-Hill, dans la craie marneuse de Douvres, dans le Detritus de Charing, dans le RedChalk de Flanborough et dans la craie blanche du Sud-Est de l'Angleterre. D'après le même Paléontologiste elle existerait aussi dans le terrain tertiaire Eocène de Braklcsham. Je viens de la rencontrer en oûtre dans le terrain crétacé supérieur de Teufelsmauer ${ }^{2}$ ) près Quedlinbourg

1) Von Münster, 1830. Jahrbuch für Mineral. etc. von Leoniard und Broxx. pag. 64.

2) Je dois à l'obligeance de Mons. le sénateur Hermaxn Roemer de Hildesheim la communication d'un ćchantillon du terrain crétacé supérieur de cette localité. 
en Westphalie; dans la craie blanche avec silex de l'Ile de Rügen ${ }^{3}$ ) et dans le calcaire Pisolitique (Etage Danien Alc. D’Orb.) du Mont-aimé (Haute-Marne) en France.

2. Cytherella munsteri Roemer spec. 1838.

PI. VIII, fig. 2, $a, b, c, d$.

Cytherina Munsteri Roemen, 1838. Neues Jahrbuch für Nineralogie etc: von Leonhard und Brons. pag. 516, pl. VI, fig. 13.

" parallela Reuss, 1844. Geognostische Skizzen aus Böhmen. Band II, pag. 217.

" " Reuss, 1845. Die Versteinerungen der Böhmischen Kreide-formation. Erste Abtheilung, pag. 16, pl. V, fig. 33.

" asperula? Reuss, 1845. Ibidem. pag. 16, tab. V, fig. 37.

"I solenoïdes? Reuss, 1846. Tbidem. Zweite Abtheilung, pag. 104, tab. XXIV, fig. 14. Cythere truncata Bosquet, 1847. Mémoires de la Saciété royale des Sciences de Lićge. tom. IV, pag. 357, pl. I, fig. $2 a-c$.

" "Bosevet, 1847. Descript. des Entom. foss. de la Craie de Maestricht. pag. 7 , pl. I, fig. $2 a-c$.

Cytherella truncata Jones, 1849. A Monograph of the Entomostraca of the Cretaceous formation of England. pag. 30 , pl. VII, fig. $35 a, b, c$.

Cytherina parallela Reuss apud Geinitz, 1850. Das Quadersandstein-gebirge oder Kreidegetirge in Deutschland. pag. 98, No. 1.

Cytherella Munsteri Bosquet, 1851. Description des Eintomostr. fossiles des terrains tertiaires de la France et de la Belgique. pag. 13, pl. I, fig. $2 a, b, c, d$.

Cytherina parallela Reuss, 1851. Foraminiferen und Entomostraceen des Kreidemergels von Lemberg (Naturwissenschäftlichen Abhandl, von Wilir. Haidingeir. Vierter Band, $1^{\text {ste }}$ Abth. pag. 48, tab. VI, fig. $1 a, b, c$.

La carapace de cette Cytherclla offre une grande ressemblance de forme avec la semence de l'Helianthus annuus, comme Mr. Rupert Jones l'a déja fait observer. Elle a des valves oblongues, arrondies en avant, obliquement tronquées en arrière; à bord inférieur droit ou presque droit dans la valve gauche et plus ou moins arqué dans la valve droite. Le dos des

1) C'est à Mr. le Dr. FrIedr. von Hagenow de Greifswald que je suis redevable de la communication de la craie de Rügen.

2) IIr. le Baron DE Ponsort de Chalons-sur-Marne m'a communiqué un échantillon de ce calcaire. 
valves est le plus bombé dans la moitié postérieure de la longueur; il se rattache aux bords supérieur et inférieur par une pente assez rapide, rejoint le bord antérieur par une pente assez douce et retombe presque perpendiculairement sur le bord postérieur. 'Toute la surface est lisse, parfois même luisante, et n'offre que 8-10 séries longitudinales de points creux excessivement petits.

Le bouclier présente une section longitudinale à contour cunéiforme obscurement pentagonal, et une section transversale à contour ovale.

Il n'est sujet qu'à de légères variations de forme, consistant dans la courbure plus ou moins forte des bords supérieur et inférieur des valves. Il arrive aussi, mais rarement, que celles-ci sont un peu étranglées vers la partie médiane de la longueur.

Parmi les échantillons des couches Eocènes des environs de Paris, il y en a qui présentent à la loupe un grand nombre de petites taches blanchâtres. Ces taches, vues au microscope par transparence, apparaissent sous forme de cercles opaques. A l'endroit qui repond au tubercule interne, les valves offrent à l'extérieur une fossette arrondie, an fond de laquelle on observe, chez certains individus, $7-8$ taches transparentes (lucid spots de Mr. Jones), dont les 4-5 supérieures sont très-rapprochées les unes des autres.

Observation. - Les Cytherina asperula et Solenoïdes Reuss, paraissent n'être que des variétés ou peut-être que des déviations individuelles de forme de la Cytherella Munsteri.

Dimensions. - Longueur 0,75 de millim., hauteur 0,50 de millim., et épaisseur 0,30 de millim.

Rapports et miférenees. - La Cytherella Munsteri, quoique se rapprochant de la précédente, et de la Cytherella compressa, se distingue très-nettement de toutes les deux.

Gisement et Localités. - Cette espèce, quoique assez repandue, est beaucoup moins abondante que la précédente. Dans le Duché de Limbourg elle se rencontre dans le Système Maestrichtien à St. Pierre, à Gronsveld, à Bemelen, à Geulhem, à Fauquemont et à VieuxFauquemont; dans le Système Sénonien (Dumont) (calcaire grossier à silex gris) à St. Pierre, et dans une couche qui paraît appartenir au Système Hervien entre Benzenrathhof et Keverberghof près Heerlen. En Belgique on la trouve dans la craie blanche avec silex à Jandrain et dans la craie blanche sans silex à Heure-le-Romain, dans le calcaire grossier à silex gris, à Ciply près Mons (Système Sénonien DuMont) et dans le Système Maestrichtien, à Sichen et à Folx-lesCaves. En Allemagne elle se trouve dans la craie supérieure de Teufelsmauer près Quedlinbourg (Westphalie). Suivant le Dr. Reuss elle existe dans le plänerkalk de Kutsehlin et de Kosstitz, et dans le plänermergel de Priesen en Bohème, ainsi que dans le Kreidemergel de Lemberg en Gallicie. D'après Mr. Jones on la rencontre dans la craie blanche du Sud-Est de l'Angleterre, dans le Detritus de Charing, dans la craie marneuse de Douvres et dans le Gault do Folkstone et de Leacon-Hill. Selon le même Paléontologiste elle existe aussi dans le terrain 
Eocène de Barton (Hants) et de Colwell-Bay (Ile de Wight) en Angleterre, et dans celui de l'Alabama (Amérique Septentrionale). Elle a été trouvée par moi dans les couches Eocènes des environs de Paris, dans un grand nombre de localités. Mr. Jones l'indique en outre dans le terrain crétacé supérieur de Balsberg, et je viens encore d'en trouver moi-même deux échantillons dans le même terrain d'Ifö en Suède. ${ }^{1}$ )

3. cytherella auricularis Bosquet spec. 1847.

Pl. IV, fig. $19 a, b, c, d$.

Cypridina auricularis Bosquet, 1847. Mlémoires de la Sociéte royale des Sciences de Liège. Tom IV, pag. 366 , pl. III, fig. $2 a-d$.

" " Bosquet, 1847. Description des Entomostracés fossiles de la craie de Maestricht. pag. 16, pl. III, fig. $2 a-d$.

Cytherella auricularis Joves, 1849. A llonograph of the Entomostraca of the cretaceons formations of England. pp. 31 et 36.

" " Bosquet, 1851. Description des Entomostracés des terrains tertiaires de la France et de la Belgique. pag. 16.

Valves à contour oblong-subtétragone, à bord antérieur obliquement sémicirculaire et marginé d'un limbe comprimé assez large; obtuses et obliquement subtronquées en arrière; leurs bords inférieur et supérieur sont presque droits, le premier est régulièrement et très-faiblement excavé dans toute sa longueur, tandis que le dernier est sinué en avant, et va rejoindre en arrière la troncature postérieure par une courbure assez brusque. La partie voûtée des valves est fortement déprimée, elle est garnie de bourrelets longitudinaux réunis aux extrémités, et dont l'ensemble offre un peu de ressemblance avec la forme d'une oreille humaine; le plus gros de ces bourrelets forme au côté pectoral la limite marginale inférieure de la partie voûtée; celle-ci est rattachée à la partie comprimée, qui borde les valves le long des côtés pectoral, postérieur et supérieur, par une pente très-rapide, et rejoint le limbe comprimé antérieur par une pente assez douce. La fossette, qui correspond au tubercule interne, est assez profonde; elle est placée au fond d'une partie concave, entre les bourrelets du dos des valves.

La carapace offre une section longitudinale à contour sub-cunéiforme, et une section transversale à contour hexagonal.

1) Je dois à l'obligeance le Mr. le Dr. Friedr. von Hagenow de Greifswald, des échantillons du terrain crétacé de cette localité, ainsi que de beaucoup d'autres. 
Dimensions. - Longueur 0,7 de millim., hauteur 0,4 de millim. et épaisseur 0,25 de millim.

IBapports et Difrérences. - Cette espèce est très-bien caractérisée et facile à distinguer de toutes ses congénères.

Gisement et Localités. - Cette Cytherella se trouve dans le Système Maestrichtien du Duché de Limbourg à St. Pierre, à Gronsveld, à Bemelen, ̀̀ Geulhem et à Fauquemont et dans le Système Sénonien (calcaire grossier à silex gris) entre St. Pierre et Petit-Lanaye. Elle se rencontre en oûtre en Belgique, dans le Système Maestrichtien à Sichen, et dans le Système Sénonien (calcaire grossier à silex gris), à Ciply près Mons. Elle est rare dans toutes ces loca ités.

4. GYTHERELla DENTiGulata nov, spec. 1853.

Pl. V, fig. $1 a, b, c, d$.

Les valves de cette Cytherella sont allongées-subtétragonales, tronquées en arrière, arrondies en avant et marginées d'un limbe assez large, et garni d'un grand-nombre de petites dentelures marginales. Leurs bords supérieur et inférieur sont presque droits et parallèles, le premier offre une faible saillie vers sa partie moyenne; une saillie plus forte est produite à l'extrémité antérieure du bord inférieur, par le prolongement du limbe comprimé antérieur. La partie voûtée des valves se rattache au limbe antérieur par une pente assez douce, aux parties comprimées "margimales des bords supérieur et inférieur par une pente très-rapide, et retombe perpendiculairement sur le bord postérieur. Sur la partie voûtée s'élèvent 3-4 bourrelets longitudinaux, dont les deux inférieurs seuls sont nettement limités; ces deux derniers sont parallèles, et se touchent dans leur moitié antérieure, tandis que dans leur moitié posterieure ils sont divergents; vers la partie moyenne des deux autres on voit la fossette, passablement profonde, qui repond au tubercule interne.

Le bouclier offre une section longitudinale à contour cunéiforme, et une section transversale à coutour hexagonal.

Dimensions. - Longueur 0,75 de millimètre, hauteur 0,4 de millimètre et épaisseur 0,25 de millimètre.

IRapports et Difrérences. - Elle se rapproche beaucoup de ma Cytherella hierogly. phica; ${ }^{1}$ ) mais s'en distingue par ses dentelures antérieures plus petites et plus nombreuses; par la direction et la forme des bourrelets de la surface, et par l'absence de la protubérance au fond de la fossette subcentrale de ses valves.

1) Bosevet, 1851. Descript. des Entonostr. foss. des terrains tertiaires de la France et de la Belgique. pp. 15, 16, pl. I, fig. $3 a-d$. 
Gisement et Localités. - Elle se trouve dans le Système Maestrichtien à St. Pierre, à Gronsveld et à Bemelen; et dans le calcaire grossier à silex gris (Système Sénonien DumonT) entre St. Pierre et Petit-Lanaye.

5. Cytherella williamsoniana Jones, 1849.

Pl. V, fig. 2 $a, b, c, d$.

Cytherina pedata? Geinitz, 1843. Versteinerungen von Kieslingswalda, und Nachtray zur Charakteristik des Sachsich Böhmischen Kreidegeb. pp.6, Taf. 5, fig. 13.

Cytherella Williamsoniana Jones, 1849. A Nonograph of the Entomostraca of the Cretaceous formation of England. pag. 31, Tab. VII, fig. $26 a-i$.

Cypridina leioptycha Reuss, 1851. Naturwissenschäftl. Abhandl. von WilH. Haidinger. Vierter Band, $1^{\text {ste }}$ Abth. pag. 49, 50, tab. VI, fig. $11 a-c$.

Cette belle Cytherella a des valves oblongues, arrondies aux deux extrémités, et munies en arrière de quelques dents espacées très-petites et très-courtes. Leurs bords supérieur et inférieur sont presque droits et faiblement divergents en avant; le premier est légèrement sinué à ses deux éxtrémités. Antérieurement elles sont marginées d'une partie comprimée. Immédiatement en arrière de celle-ci, on voit une partie relevée en forme de bourrelet. Cette partie est lisse, parallèle au bord, et disparait vers les extrémités antérieures des bords supérieur et inférieur; une élevation pareille, mais plus forte et plus haute, se remarque le long des bords postérieur et inférieur; celle qui se trouve le long du côté postérieur, prend naissance à côté de l'extrémité postérieure du bord inférieur, et va s'effacer vers la partie médiane des valves; celle que l'on remarque le long du bord inférieur est faiblement arquée; elle a son origine vers le tiers antérieur et médian des valves; et se termine un peu en avant de l'extrémité inférieure de celle qui borde le côté postérieur. Les dépressions, qui se trouvent entre ces trois bourrelets, sont couvertes de petits tubercules. La cavité interne des valves est très-peu profonde, et le tubercule interne peu proéminent. Au fond de la fossette externe qui repond à ce dernier, on remarque une protubérance analogue à celle que présentent, au même endroit, les valves de la Cytherella hieroglyphica.

Les tubercules qui oment les dépressions de la surface de cette espèce, chez les individus parfaitement adultes, s'effacent plus ou moins dans les vieux individus. Chez ces derniers on observe en oûtre, qu'aux angles supérieur et inférieur de l'extrémité postérieure de chaque valve, le bourrelet marginal est fortement renflé, de manière qu'en ces endroits, ce bourrelet offre deux grosses protubérances. Mr. Jones a observé de très-jeunes individus, ayant les valves presque ovales. 
Fapports et Différences. - Cette espèce présente des caractères très-remarquables et est facile à distinguer de toutes ses congénères connues.

Dimensions. - Longueur 0,8 de millim., hauteur 0,5 de millim. et épaisseur 0,3 de millim.

Gisement et Localités. - J'ai trouvé cette interessante Cytherella dans le Duché de Limbourg (dans le Système Hervien?) entre Benzenrathhof et Keverberghof près Heerlen; dans la craie blanche sans silex (Système Sénonien Dowont) à Slenaken, à Pesaken et à Beutenaken; en Belgique elle se trouve dans le même Système crétacé de Jandrain et de Heure-le-Romain. Elle n'est pas rare dans cette dernière localité. - Elle a été trouvée en Angleterre par Mr. Jones, dans le Gault de Folkstone et de Leacon-Hill, dans la craie marneuse de Douvres, dans le detritus de Charing et dans la craie blanche de Thorpe près Norwich et dans celle de Gravesend, dans le Sud-Est de l'Angleterre. Le Dr. Reuss a signalé cette espèce comme étant rare dans le Kreidemergel de Lemberg en Gallicie.

Gen. II. Bambia M'Cor, 1844 .

Cytheres spec. von MUNSTER, 1830. Jahrbuch für Mineralogie und Geologie von LEONHARD und BRoN, , pp. 62 et suivantes.

Cytherinae spec. Fr. Ad. Rowyer, 1838. Ibidem. pp. 514-519.

" spec. Fr. AD. Roemer, 1840. Versteinerungen des Nord-deutschen Kreide-gebirges. pp. 104, 105.

Bairdia M'Cor, 1844. Synopsis of the Charact. of the Carboniferous limestone fossits of Ireland. Cytherinae spec. Reuss, 1845, 1846. Die Versteinerungen der Böhmischen Kreide-formation, $1^{\text {ste }}$ Abtheilung, pag. 16, und $2^{\text {te }}$ Atheilung, pp. 104, 105.

Cytheres spec. Bosquet 1847. Mémoires de la Société Royale des sciences de Liége. tom. IV, pp. $356-358$.

spec. Bosquit, 1847. Description des Entomostracés fossiles de la craie de Maestricht. pp. 6-8.

spec. Brons, 1848. Index Palaeontologicus. - Oder Uebersicht der bis jetzt bekannten fossilen Organismen. pp. 395, 396.

spec. Connueu, 1849. Description de nouveaux fossiles microscopiques du terrain crétacé infér. du Departement de la Haute-Marne (Mëm. de la Soc. Géolog. de France, 2 $2^{\text {me }}$ Série, tom. III, pp. 241-246).

Cytherinae spec. Reuss, 1849. Die fossilen Entomostraceen des Oesterreichischen tertiërbeckens, pag. 7 et suivantes. (Aus den Naturwissenschüftlichen Abhandlungen von Wilheld Haidinger, III Band, $1^{\text {ste }}$ Abtheilung). 
Cythere (Sub-genus Bairdia Jones) 1849. A Monograph of the Entomostraca of the Cretaceous formation of England, pp. 22-27. (Mémoirs of the Palacontograplical Society).

Cytherinae spec. Geinimz, 1850. Das Quadersandstein-gebirge oder Kreide-gebirge in Deutschland, Zweite Hälfte, Erste Lieferung, pag. 98.

Bairdia Bosquet, 1850. Description des Entomostr. fossiles des terrains tertiaires de la France et de la Belgique. pp. 18-37.

Cytherinae spec. Rzuss, 1851. Die Foraminiferen und Entomostraceen des Kreidemergels von Lemberg (in den Naturwissenschäftlichen Abhandlungen von Winw. Haidinger. Vierter Band, $1^{\text {ste }}$ Abth. pp. 47-49).

Caractères Génériques. - Carapace de consistance cornée ou corneo-calcaire, formée de deux valves inégales, trigones, ovales ellipsoïdales, réniformes ou mytiliformes, et plus ou moins convexes. La surface extérieure de ces valves est lisse, ou creusée de points plus ou moins nombreux, ou garnie d'épines, le plus souvent extrémement minces, piliformes ou aciculaires. Les bords sont fréquemment transparents et montrent alors dans leur épaisseur des stries blanchâtres dirigées du centre vers la circonférence.

La valve gauche des Bairdia est constamment plus grande que la valve droite et embrasse fortement les bords supérieur et inférieur de cette dernière. La charnière dorsale est formée sur la valve gauche d'un sillon longitudinal qui, vers le milieu du bord cardinal, devient ordinairement si étroit, qu'il paraît presque totalement effacé. Le bord dorsal de la valve droite est plus étroit que celui de la valve gauche, et s'insére nettement dans le sillon du bord correspondant de cette dernière.

Les bords internes antérieur, inférieur et postérieur de la valve droite des Bairdia sont convexes, tandis que les bords correspondants de la valve gauche sont concaves, ou obliquement inclinés vers le centre. Lors de la rémion des deux valves, les bords convexes de la première viennent se placer contre les bords concaves de la dernière.

L'arête interne du bord valvaire est garnie le long des côtés antérieur, inférieur et postérieur, d'une lame qui est toujours très-mince et le plus souvent très-étroite, mais qui, chez certaines espèces, par exemple chez les Bairdia linearis, arcuata, siliqua etc. acquiert un tel développement, et fait une saillie si forte vers l'intérieur aux deux extrémités des valves, que des cavités profondes se produisent entre elle et la surface interne.

Ces deux cloisons internes aux extrémités des valves existent aussi chez les Candona.

Le bord inférieur des deux valves des Bairdia est ordinairement infléchi un peu en avant 
du milieu comme chez les Cythere et les Cytheridea; il est en même temps un peu plus étroit que partout ailleurs; de sorte que la partie interne concave ou bien devient très-étroite en cet endroit, ou bien s'efface totalement. Il est souvent un peu saillant en ce même endroit il resulte de cette disposition que, quand les deux valves sont réunies, leur ligne de jonction n'est pas droite, mais offre un petit prolongement ou lobe saillant sur la valve droite. Cette lamelle aiguë, la lame pectorale de Mr. Connoer, est plus ou moins développée suivant les espèces. Elle sert à fermer plus complètement les deux valves, parceque celle de la valve droite s'engage sous celle de la valve gauche.

La paroi intérieure de chaque valve des Bairdia présente constamment une petite fossette arrondie, très-peu profonde et située, non pas sur la ligne longitudinale médiane, mais entre cette ligne et le côté pectoral, vers le tiers antérieur de la longueur totale des valves.

Cette petite fossette interne, qui est assez souvent presque imperceptible, ne correspond que très-rarement à une partie saillante externe chez les Bairdia; mais dans les espèces vivantes et dans les échantillons fossiles qui ont conservé leur transparence, on remarque au microsope, dans l'épaisseur même des valves, ì l'endroit oû se trouve cette petite fossette interne, un assemblage de taches arrondies ou anguleuses, plus transparentes que le reste. Le nombre de ces taches transparentes, leur forme et leur disposition, paraissent varier dans les diverses espèces; elles ont une analogie parfaite avec l'assemblage de taches que l'on. observe constamment sur les deux valves des Cypris et des Candona. Cet assemblage de taches, dont on ne connait pas encore la nature et l'usage, est remplacé par un tubcrcule interne chez les Cytherella, et par une fossette interne, ordinairement assez profonde, et repondant très-souvent à un tubercule externe, chez les Cythere.

Le genre Bairdia offre un assez grand nombre de répresentants fossiles. Les terrains Paléozoïques n'en renferment cependant qu'un nombre tres-limité d'espéces; on ne peut en signaler que sept à huit de la formation crétacée; il atteint un assez grand développement spécifique dans les divers Systèmes du terrein tertiaire, et parnît avoir encore actuellement un bon nombre de representants vivants. Je n'en connais jusqu’à présent que trois des couches crétacées du Duché de Limbourg.

1. bairdia subglobosa Bosquet, 1850 .

Pl. VIII, fig. $3 a, b, c, d$.

Bairdia Subglobosa Bosquet, 1850. Description des Entomostracés fossiles des terrains tertiaires de la France et de la Belgique. pag. 23, pl. I, fig. 7 a-d.

Valves lisses, très-enflées, offrant un contour ovale oblique. Elles sont arrondies aux 
extrémités et leur bord dorsal est assez fortement arqué. Elles sont tellement enflées au côté pectoral, que leur voûte dorsale dépasse le bord, et que ce côté semble très-fortement arqué. Leur surface offre un grand nombre de points creux très-petits, dans lesquels ont probablement été insérés des poils ou plutôt de petites épines aciculaires.

La carapace presente une section longitudinale à contour ovale, et une section transversale à contour subcirculaire.

Dimensions. - Longueur 0,6 de millim., hauteur 0,4 de millim. et épaisseur 0,45 millim.

Rapports et Bifférences. - Cette Bairdia se distingue nettement de toutes les autres; par ses valves raccourcies et excessivement enflées.

Gisement et cocalités. - Elle n'est pas rare dans le Système Maestrichtien du Duché de Limbourg à Nedercanne, à St. Pierre, à Bemelen et à Fauquemont. Elle se rencontre dans le même Système Crétacé à Ciply près Mons en Belgique. Elle se trouve en oûtre en France dans le terrain tertiaire (étage Suessonien D'Orb.) de Ménilmontant; dans l'étage Parisien A ALc. D’Orb. de Grignon, de la Ferme de l'Orme, de Chamery, de St. Felix, du Vivray, de Chaumont, de Chateaurouge, de Parnes et de Courtagnon; dans l'étage Parisien B, de Guépesle et de Pisseloup et dans l'étage subapennin ALC. D’Orb. de Perpignan (Pyrénées orientales).

2. Bairdia subdeltoidea von Munster spec, 1840.

Pl. VIII, fig. $4 a, b, c, d, e, f$.

Cythere subdeltoïdea von Münsten, 1830. Jahrbucle für Mineralogie wnd Geologie von LeONHARD und Brons. pag. 64.

" " von MüNster, 1835. Tbidem. pag. 446.

Cytherina subdeltoïdea Fr. AD. Roemer, 1838. Ibidem. pag. 517, pl. VI, fg. 16. voN HAUER, 1839. Ibidem. pag. 429.

" Vor Haven, 1839. Ibidem. pag. 420.

" Fr. Ad. Roemer, 1840. Die Versteinerungen des Nord-deutschen Kreidegebirges. pag. 105, pl. XVI, fig. 22.

" Geinitz, 1842. Charakteristik der Schichten und Petrefakten des Sachsich-Böhmischen Kreidegebirges. $3^{\text {te }}$ Abth. pag. 64.

" " Reuss, 1845. Die Versteinerungen der Böhmischen Kreide-formation. Erste Abth., pag. 16, pl. V, fig. 38, und $2^{\text {te }}$ Abth. pag. 104.

GeInixz, 1845. Grundriss der Versteinerungkunde. pag. 244, pl. VIII, fig. 21.

" trigona Bosedet, 1847. Memoires de la Société royale des Sciences de Liége. pag. 358, pl. I, fig. $3 a-e$. 
Cythere trigona Bosavet, 1847. Description des Entomostracés fossiles de la craie de Maestricht. pag. 8 , pl. I, fig. $3 a-e$.

" (Barrdis) subdeltoïdea Jones, 1849. A Monograph of the Entomostraca of the cretaceous formation of England. pag. 23, pl. V, fig. $15 a-f$.

Cytherina subdeltoïdea Reuss, 1849. Die fossilen Entomostraceen des Oesterreichischen Tertiärbekens. pag. 9, pl. VIII, fig. $1 a, b$.

Bairdia subdeltoidea Bosquet, 1850. Descript. des Entom. fossiles des terrains tertiaires de la France et de la Belgique. pag. 29, pl. I, fig. $13 a-d$.

Cytherina subdeltoidea Reuss apud GeINITz, 1850. Das Quadersandstein-gebirge oder Kreidegebirge in Deutschland. pag. 98, No. 10.

" " Ed. ron EICHWALd, 1852. Lethea Rossica, ou le monde primitif de la Russie. pl. XI, fig. $23 a-d$.

" Naumann, 1852. Lelerbuch der Geognosie. Zweite Hälfte, taf. LX, fig. 24 .

" " Reuss, 1851. Naturwissensch. Abhandl. von Wili. Haidinger. Vierter Band, $1^{\text {ste }}$ Abtheilung, pag. 47.

Valves fortement bombées, ovales-subtrigones, et pointues en forme de bec en arrière. Leur bord supérieur est très-fortement arqué; l'inférieur est arqué-ellipsoidal et infléchi vers le milieu, principalement sur la valve droite, qui offre souvent en cet endroit un sinus très-sensible. Dans cette dernière, qui est toujours plus petite que la valve gauche, le bord dorsal est tronqué de telle manière, qu'il présente un contour semblable à la moitié d'un hexagone régulier. La surface des deux valves est luisante; elle est recouverte, pendant la vie, d'un très-grand nombre de longs poils, qui manquent constamment sur les échantillons fossiles. Ces poils sont insérés dans des points creux excessivement petits et qui s'observent sur les échantillons fossiles à l'aide du microscope. Très-rarement les deux valves offrent, à chacune de leurs extrémités, $4-5$ épines longues et droites, ${ }^{1}$ ) et selon Mr. Joves, certains individus présentent, à la surface des deux valves, plusieurs épines espacées et assez fortes.

Les valves sont tellement bombées au milieu, que la carapace offre en cet endroit une section transversale à contour suborbiculaire.

1) Ces épines ont été observées par Mr. JoNes, sur des individus fossiles de sa collection, et sur des individus vivants recueillis à Manille et à Tenedos, et se trouvant dans la collection de Mr. Wriliamson. _ J'ai rencontré quelques échantillons pareils dans le terrain crétacé Maestrichtien, et dans le terrain sub-apennin de Perpignan en France. 
Sur les échantillons récents, qui ont ordinairement une couleur rose-purpurine, on remarque vers la partie médiane du dos des valves un espace opaque, de forme et de grandeur irrégulières. Dans la partie inférieure de cette région opaque, et un peu en dessous du centre de chaque valve, on voit distinctement, à l'aide du microscope, la rosette transparente qui a été observée en premier lien par Mr. R. Jones. Cette rosette se compose de 5-6 taches transparentes et arrondies-oblongues, placées autour d'une tache centrale, plus petite et arrondie. La place occupée par chacune de ces taches transparentes est concave à l'extérieur, et faiblement proéminente à l'intérieur des valves, et les petites proéminences internes sont situées au fond d'un espace concave plus grand que la rosette elle-même. Cet espace concave s'observe en général plus facilement sur les échantillons fossiles, que sur la plupart des échantillons récents.

Bimensions. - Longueur 1,4 de millimètre, hauteur 0,9 de millimètre et épaisseur 0,7 de millimètre.

Tiapports et Minérences. - Elle présente une forme si bien caractérisée, qu'elle ne peut être confondue avec aucune de ses congénères connues.

Gisement é Localités. - Cette Bairdia est, parmi tous les Ostracodes vivants et fossiles connus, l'espèce qui est la plus répandue dans presque toutes les couches crétacées et tertiaires. Elle existe en oûtre encore actuellement à l'état vivant.

Je l'ai trouvée dans le Duché de Limbourg, dans le psammite glauconifère (Système Hervien Duront) à Simpelveld, à Slenaken et à Pesaken; dans le même? Système, entre Benzenrathhof et Keverberghof près Heerlen; dans la craie blanche avec silex au Nord de Wahlwiller, dans la craie blanche sans silex à Slenaken, à Beutenaken et entre Vaals et Gimenich; dans le calcaire grossier à silex gris (Système Sénonien Domont) entre St. Pierre et Petit-Lanaye; et dans le Système Maestrichtien à Nedercanne, à St. Pierre, à Gronsveld, à Keer, à Bemelen, à Geulhem, à Fauquemont, à Kunraede et à Bulkomsdal. Je l'ai rencontrée en Belgique, dans le psammite glauconifère (Système Hervien Dumoñ) près Gimenich et à Visé; dans la craie blanche de Jaudrain et de Heure-le-Romain, et dans le calcaire grossier à silex gris (Système Sénonien Dumont) de Frère près 'Tongres; ainsi que dans le Système Maestrichtien de Sichen, de Sussen, de Jandrain, de Folx-les-Caves et de Ciply près Mons.

Elle se trouve encore dans le calcaire Pisolithique (ètage Danien d'Orbigny) du Mont-aimé (Haute-Marne) en France; dans le terrain Danien de Faxoë en Danemarck, et dans le terrain Maestrichtien d'Ifö (Schoonen) en Suède. D'après Mr. Jones elle se trouve en Anggleterre, dans le Greensand de Warminster, dans le Chalk-marl de Douvres, dans le Detritus de Charing et dans la craie blanche du Sud-Est de l'Angleterre. D'après Mr. Fr. Ad. Roemer elle se rencontre dans la craie marneuse inférieure de Lemförde; suivant le Dr. Gernitz dans le pläner- 
kalk de Strelen près Dresde et selon le Dr. Reuss dans le plänerkalk et plänermergel de la Bohème et dans le Kreidemergel de Lemberg en Gallicie, enfin, je viens d'en trouver quelques échantillons dans la craic supérieure de Teufelsmauer près Quedlinbourg (Westphalie) en Allemagne, et dans celle de la Nouvelle-Jersey (Etats-Unis).

Dans les terrains tertiaires de la France elle a été trouvée en oûtre par moi:

dans l' Etage Suessonien d'Orbignt, d'Epernay, de Soissons, de Ménilmontant, de Cuise-la-Mothe; dans l'Etage Parisien A; D’Orв. de Chamery, de Grignon, de la ferme de l'Orme, de Houdan, du Vivray, de Chambord; de St. Felix, de Chaumont, du mont Ganelon, de Châteaurouge, de Parnes et de Montmirail;

dans l'Etage Parisien B; D’Orb., de Guépesle, de Ver, de Pisseloup;

dans l'Etage Tongrien (Falunien) D'OrB., de Jeurre et d'Etrechy;

dans l'Etage Falunien D’Orb., de Léognan. de Dax, de Mérignac, et

dans l'Etage subapennin n’Orв., de Perpignan.

Stuivant le Dr. Reuss elle se trouve en Autriche:

à Nussdorf, à Steinabrunn, à St. Nicolaï, à Wurzing, à Freibuhl (Styrie) à Kostel (Moravie), à Rust (Hongrie) et à Rudelsdorf (Bohème); et

en Italie: à Castell' Arquato.

Selon Mr. R. Jones, elle existe en Angleterre:

dans le terrain Pliocène de Sutton et de Walton, et dans le terrain Eocène de l'Ile de Wight.

D'après le même Naturaliste elle se rencontre aux Etats Unis:

dans le terrain Miocène de la Virginie et de Valparaiso;

Mr. Frid. Sandeerger vient de la découvrir dans le meeressand de Weinheim, près Mayence.

Mr. Reuss l'indique encore dans le Nord-Ouest de l'Allemagne. A l'état vivant elle existe dans la Méditerranée, et selon Mr. Jones près Sydney (Nouvelle Hollande); à Providence et à Turk's Island (Bahama) à l'Ile Maurice, à Manille et au Nord de l'Angleterre.

2. Bairdia arguata von MüNster spec. 1830.

Pl. V, fig. $3, a, b, c, d$.

Cythere arcuata von Müssten, 1830. Jahrbuch für Ifineralogie und Geologie von Leonhard und Brows. pag. 63.

" " von MüNster, 1835. Ibidem. pag. 446.

Cytherina arcuata Fr. AD. RokMer, 1838. Ibidem. pag. 517, pl. VI, fig. 17.

" " Philippi, 1844. Beitr. zür kenntn. der Tertiär-Verstein. des Nord-Westl. Deutschlands. pag. 63. 
" faba? Reuss, 1846. Versteinerungen der Böhmischen Kreide-formation. Abtheil. II, pag. 104, tab. XXIV, fig. 13.

Cytherina arcuata Reuss, 1849. Die fossilen Entomostraceen des Oesterreichischen Tertiärbeckens. pag. 11, pl. VIII, fig. $7 a, 8$.

Bairdia siliqua var. a. Jones, 1849. A Monograph of the Entomostraca of the Cretaceous formation of England. pag. 25, pl. VI, fig. $16 e, f, g$.

" triquetra? Reuss, 1849. Ibidem. pag. 27, pl. VI, fig. $19 a-c$.

" arcuata Bosquer, 1550. Description des Entomostracés fossiles des terrains tertiares de la France et de la Belgique. pag. 32, pl. I, fig. 14a-d.

Cytherina acuminata A七th. 185̃1. Naturw. Abhandl. von WilH. Haidinger. IV Band, $1^{\text {ste }}$ Abth. pag. 49, tab. VI, fig. 7,8.

VAR. B. GRAculs. - Valvis multo angustioribus, longitudinis altitudine fere quadruplo majoris. BosQUET. Pl. V, fig. $4 a, b, c, d$.

Cytherina modesta Reuss, 1851. Naturwissensch. Abhandl, von WiLH. Haidinger. Vierter Band, $1^{\text {ste }}$ Abtheil. pag. 49, tab. VI, fig. $9 a, b, c$.

VAR. $G$. LUNATA. - Valvis multo angustioribus, in dimidia parte anticâ curvatis; unde formae sub-lunatae Boseuet.

Cytherina lunata Fr. AD. Roener, 1838. Neues Jahrbuch für Mineralogie etc: von LEoNhaRD und BRonn. pag. 517, pl. VI, fig. 18.

Les valves de cette Bairdia sont allongées-subtriangulaires, presque $2^{1 / 2}-3$ fois aussi longues que larges, convexes, arrondies en avant et plus ou moins pointues en arrière. Leur bord supérieur est assez fortement arqué, subanguleux vers le milieu; l'inférieur est faiblement arqué, presque droit et légèrement infléchi au milieu. Leur surface luisante offre au microsope plusieurs points creux très-petits, qui paraissent être les points d'insertion des très-petites épines aciculaires ou des poils que Mr. Jones a observés sur les échantillons récents. Chez certains individus de l'Etage Falunien de Bordeaux et de l'Etage Parisien, chacun des points creux qui se trouvent vers le centre des valves, est entouré d'une petite tache brune.

La carapace présente une section longitudinale à contour ovale-allongé, et une section transversale à contour ovale-arrondi.

Les échantillons des dépôts crétacés sont un peu moins convexes aux extrémités, un peu plus étroits dans leur moitié postérieure, et sont terminés en arrière en une pointe un peu plus aiguë que ceux qui proviennent des couches tertiaires.

La variété B n’a été trouvée jusqu'ịci que dans le terrain crétacé de Kunraede (Duché de 
Limbourg) et parait être fort rare. La variété G se trouve, d'après Mr. Fr. AD. Roemer, dans le terrain sub-apennin de Castell' Arquato en Italie.

Dinansions. - Longneur 1,10 de millimètre, hauteur 0,6 de millimètre et épaisseur 0,45 de millim. La variété $B$ a une longueur de 1,05 de millim. et une hauteur de 0,35 de millim.

Observation. - J'ai reçu dernièrement de Mr. R. Jones des échantillons de sa Bairdia siliqua. $\left.{ }^{1}\right)$ Après les avoir comparés soigneusement avec ceux de la Bairdia arcuata, je suis d'avis qu'ils constituent une espèce bien distincte, dont les dimensions sont constamment plus grandes que celles de cette dernière et qui devra conserver la nom que Mr. Jones lui a donné. Je pense au contraire, que les échantillons figurés et décrits par le même Naturaliste comme variété \& de sa B. Siliqua, devront être rapportés à la B. arcuata, et qu'ils sont exactement les mêmes que ceux qui se trouvent dans les couches crétacées des environs de Maestricht.

Gisement et Localités. - Elle se rencontre rarement dans le Système Maestrichtien à St. Pierre, à Gronsveld, à Bemelen, à Geulhem, à Fauquemont et à Kunraede; dans le calcaire grossier à silex gris (Système Sénonien Dumont) à St. Pierre et dans le Système Hervien? entre Benzenrathhof et Keverberghof près Heerlen. Je l'ai trouvée en Belgique dans le Système Maestrichtien de Sichen, de Wonck et de Folx-les-Caves.

J'en ai observé aussi quelques valves dans le terrain Maestrichtien d'Ifö (Schoonen) en Suède, et dans la craie supérieure de la Nouvelle Jersey (Etats Unis).

D'après Mr. Jones la Bairdia areuata se trouve dans le Detritus de Charing, et à l'état vivant près Ténedos.

Elle a été trouvée en oûtre dans l'Etage Falunien ALc. D'OrB. à Dax, à Léognan, et à Mérignac; dans l'Etage Tongrien à Jeurre, et à Etréchy, près d'Etampes; dans l'Etage Parisien, à Chaumont, à Grignon et à la Ferme de l'Orme, et dans l'Etage Suessonien à Cuise-la-Mothe. En Belgique je l'ai trouvée dans le Système Bruxellien Domont, de St. Josse-ten-Noode. Le Dr. Reuss l'a trouvée en Autriche, dans le leithakalk de Nussdorf et de Kostel en Moravie, dans le tegel de Rudelsdorf en Bohème et de Grinzing près Vienne, dans le sel gemme des Salines de Wieliczka en Gallicie, et rarement dans le tegel de Möllersdorf près Baden. Suivant le même Naturaliste elle se trouve dans le terrain subapennin de Castell' Arquato près Parmes en Italie. D'après Mr. Fr. Ad. Roemer dans le terrain tertiaire d'Osnabruck en Westphalie, et suivant Mr. Phimipri près Freden, dans le Nord-Ouest de l'Allemagne. Mr. Frid. SAndberger enfin, vient de la trouver dans le meeressand de Weinheim, dans le bassin tertiaire de Mayence.

1) Jones, 1849. A MTonograph of the Entom. of the Cretac. form. of England. pag. 25, pl. V, fig. 16 a, $b, c, d$. (exclusis alteris). 


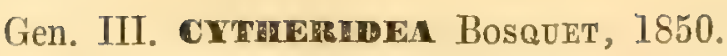

Cytheres spec. von Münster, I830. Jahrbuch für Mineralogie und Geologie von Leoninad und Brons. pag. 62 et suivantes.

Cytherinae spec. Bronn, 1838. Index Palaeontologicus. Uebersicht der bis jetzt bekannten fossilen Organismen. pag. 396.

Cytherinae spec. Reuss, 1849. Die fossilen Entomostraceen des Oesterreiclischen Tertiürbeckens. pag. 7 et suivantes. (Aus den Naturwissenschäftlichen Abhandlungen von Vilmedir Haidinger. III Band, $1^{\text {ste }}$ Abtheilung).

Cytheres et Bairdiae snec. Jones, 1849. A Monograph of the Entomostraca of the cretaceous formation of England. pp. 1-13 et pp. 25, 26. (Mémoirs of the Palaeontographical Society).

Caractères Gémériques. - J'ai établi en 1850 le genre Cytheridea, pour y grouper des Ostracodes, qui ont une grande ressemblance avec les Bairdia, mais dont l'articulation dorsale est parfaitement distincte.

La charnière des Cytheridea (qui se rapproche un peu de celle des Nucules et des Pétoncles) est formée, sur la valve droite, de deux séries de $6-8$ petites dents, égales en grandeur, insérées sur deux parties tant soit peu saillantes des extrémités de l'étroit bord dorsal, ou plutôt de la barre cardinale de cette valve, et correspondant à deux séries de petites fossettes, placées sur une partie abaissée du côté interne du bord cardinal de la valve opposée. (Voyez mon Mémoire sur les Entomostracés fossiles des terrains tertiaires de la France et de la Belgique. pl. II, fig. $4 b$ et $c$, et la Monographie de Mr. Jones des Entomostracés des terrains crétacés de l'Angleterre. pl. I, fig. 1, $c$ et $d$ ).

Chez la plupart des espèces de ce Genre, on remarque sur chaque valve, près de l'extrémité antérieure du bord supérieur, un petit tubercule circulaire, luisant comme du verre, et analogue à celui qu'on observe à peu près au même endroit, sur chaque valve des Cythere.

Je connais actuellement treize espèces qui se rapportent au Genre Cytheridea. Trois de ces espèces proviennent de la formation crétacée; toutes les trois ont été trouvées dans les terrains du Duché de Limbourg, et deux d'entre elles avaient déja été décrites par Mr. R. Jones, ct rencontrées par lui dans les dépôts crétacés de l'Angleterre. Les dix autres proviennent des terrains tertiaires. Parmi ces dernières, la Cytheridea Mulleri nous offre encore son représentant vivant dans nos mers actuelles, et suivant Mr. Jones la Cytheridea (BAIRDIA) Harrisiana de la période crétacée, aurait également son représentant vivant sur les côtes de Manille. 
1. CYTHERIdeA harkisiana Bosquet, 1853.

Pl. $V$, fig. $5 a, b, c, d$.

Bairdia Harrisiana JonEs, 1849. A Monograph of the Entomostraca of the cretaceons formation of England. pp. 25, 26, pl. VI, fig. $17 a-f$.

Valves allongées, arrondies en avant, sub-aiguës, un peu obtuses et obliques en arrière. Leurs bords supérieur et inférieur sont faiblement arqués. Leur voûte dorsale est fortement convexe; sa plus grande convexité est un peu en arrière de la moitié de la longueur totale. De cet endroit, elle se rattache aux deux extrémités par une pente assez douce. La surface est lisse, mais, selon Mr. Jones, il y a des échantillons dans lesquels elle est creusée de points et couverte d'épines.

La carapace offre une section longitudinale à contour ovale-comprimé, et une section transversale à contour ovale-arrondi.

Observation. - J'ai cru devoir ranger cette espèce dans mon genre Cytheridea, parceque Mr. Jones (qui l'a trouvée assez abondamment dans le detritus de Charing) a observé, sur le bord dorsal de la valve droite, une série de fines crénelures (ou dentelures).

Dinensions. - Longueur 0,6 de millim., hauteur 0,25 de millim. et épaisseur 0,2 de millin.

Gisement et Localités. - Je n’ai trouvé jusqu'ici que deux échantillons bivalves de cette espèce; l'un dans le terrain crétacé Maestrichtien de Kunraede près Heerlen, et l'autre dans celui de Gronsveld. Elle a été observée par Mr. Jones dans le Speeton-clay de Yorkshire, dans le gault de Leacon-Hill, dans le Detritus de Charing, et dans la craie blanche de Gravesend et de Charlton. Suivant le même Paléontologiste, elle existerait aussi dans le calcaire carbonifère de l'Est de Kilbride près Glasgow, et l'on en rencontrerait, à l'état vivant, une variété finement velue à Manille, Philippines). ${ }^{1}$ )

2. CYTHERIDEA OVATA nov. spec. 1853.

Pl. V, fig. $6 a, b, c, d$.

Valves lisses, obliquement ovales, arrondies aux deux extrémités, rétrecies en avant. Leur voûte dorsale est le plus convexe un peu en arrière de la moitié de la longueur totale; de ce point, elle se rattache aux deux extrémités par une pente assez douce, et aux bords dorsal et pectoral par une pente rapide.

1) Collection de Mr. Williamson. 
La carapace présente une section transversale à contour ovale-arrondi, et une section longitudinale ì contour ovale-subrhomboïdal.

Dinensions. - Longueur 1 millim., hauteur 0,6 de millim. et épaisseur 0,4 de millim.

Tapports et Dirférences. - Elle se distingue au premier abord de la suivante par ses valves lisses et toujours plus étroites en avant qu'en arrière.

Gisement et Localités. - Cẹte Cytheridea est assez rare dans le Système Maestrichtien à Gronsveld, à Heer, à Bemelen, à Geulhem, à Fauquemont et à Kunraede.

3. GyTHERIdeA JONESIANA Bosquet, 1850 .

Pl. VIII, fig. $5 u, b, c, d$.

Cythere Hilseana Jones, 1849 (non RoEmer). A Monograph of the Entomostraca of the cretaceous formation of England. pp. 10, 11, pl. I, fig. $1 a-g$.

Cytheridea Jonesiana Bosovet, 1850. Description des Entomostracés fossiles des terrains tertiaires de la France et de la Belgique. pag. 38.

Les valves de cette Cytheridea sont ovales-subtrigones; leur bord antérieur est obliquement sémicirculaire, le postérieur est subanguleux et tourné vers le côté pectoral. Leur voûte dorsale est convexe et à surface creusée d'un très-grand nombre de points, dans lesquels sont insérés des poils extrémement fins et courts. Certains individus présentent, sur la moitié inférieure des bords antérieur et postérieur, quelques dents courtes.

La carapace offre une section longitudinale à contour ovale, aigu aux extrémités, et une section transversale à contour ovale-arrondi.

Dimensions. - Longueur 0,9 de millimètre, hauteur 0,6 de millimètre et épaisseur 0,5 de millimètre.

Rapports et Différences. - Ayant eu l'occasion de pouvoir comparer les échantillons du Hilsthon du Hils, décrits sous le nom de Cytherina Hilseana par Mr. Fr. Ad. Roemer ${ }^{1}$ ) avec ceux du gault et de la craie marneuse d'Angleterre, qui ont été décrits sous ce même nom spécifique par Mr. Jones (et que je dois à l'obligeance de l'Auteur de la belle Monographie des Entomostracés du terrain crétacé de l'Angleterre), je puis affirmer que ce sont deux espèces différentes, comme je l'avais déja avancé en 1850 .

Gisement et Localités. - Cette espèce est assez rare dans le calcaire Maestrichtien de Kunraede. D'après MIr. Jones elle se trouve en Angleterre dans le greensand de Blackdown,

1) FR. AD. RoEMER, 1S40. Versteinerungen des Nord-deutschen Kreide-gebirges. pag. 104. 
en abondance dans le gault de Folkstone et de Leacon-Hill et dans le Chalk-marl de Douvres. Suivant le même Paléontologiste elle se trouverait aussi dans le terrain tertiaire Eocène de Colwell-Bay, Ile de Wight, dans la formation dite d'eau douce inférieure de la même Ile, et dans l'argile de Barton (Hants); ainsi que dans la craie supérieure de Balsberg en Suède.

Gen. IV. CYTHEItE MüLten, 1785.

Cythere MüLter, 1785. Entomostraca seu insecta testacea, ctc. pp. 63-65.

Monoculus Girenin, 1791. Systema Naturae. Editio 13.

Manuei, 1792. Encyclop. Méthodique. tom. VII.

Cytherina Lamarck, 1818. Histoire naturelle des animaux sans vertèbres. $1^{\text {re }}$ edit. tom. V, p. 125. Cytherinae spec. RoEmen, 1841. Die Versteinerungen des Nord-deutschen Kreide-gebirges. pp. $104,105$.

Cypridinae? spec. De Koninck, 1841. Crustacés fossiles de Belgique. pp. I7, 19.

1842-1844. Description des animaux fossiles du terrain carbonifère de Belgique. pp. 586-588.

Cytherinae spec. Phumpri, 1844. Beitrïge zur Kenntniss der Tertiür-versteinerungen des NordWestlichen Deulschlands. pp. 62, 63.

Cytheres spec. M'Cor, 1844. Synopsis of the Charact. of the Carboniferons limestone fossils of Ireland.

" "Conver , 1846. Description des Entomostracés fossites du Departement de la Ilaute-Marne (Mém. de la Soc. Géolog. de France, $2^{\text {me }}$ Série, tom. I, $2^{\text {me }}$ partie, pp. 195-205).

Cytherinae spec. Reuss, 1846. Die Versteinemungen der Böhmischen Kreide-formation, 2 $2^{\text {te }}$ Abth. pp. 104-106.

"Willianson, 1847. Transact. Manchest. Literary and Philosoplical Sociely. Vol. VIII.

Cypridinae spec. Bosquet, 1847. Mémoires de la Société royale des Sciences de Liége. tom. IV, pp. 295 et suivantes.

" " Bosquet, 1847. Descript. des Entomostracés foss. de la craie de Maestricht. pp. 9 et suivantes.

" et Cytheres spec. Brons, 184.S. Index Palaeontologicus. - Oder Uebersich der bis jetzt bekannten fossilen Organismen. pp. 357, 395, 396.

Cypridina Reuss, 1849. Die foss. Entomostraceen des Oesterreichischen Tertiärbeckens. pp. 21-47. Cytheres spec. Connuel, 1849. Description de nouvenux fossiles microscopiques du termin 
crétacé inférieur du Départ. de la Haute-Marne (Mem. de la Soc. Géolog. de France. $2^{\text {me }}$ série, tom. III, pp. 241-246).

Cythere et Sub-Genus Cythereis Jones, 1849. A Monograph of the Entomostraca of the cretaceous formations of Eingland. pp. 8-22.

Cypridina Reuss apud GeINITz, 1850. Das Quadersandstein-gebirge oder Kreide-gebirge in Deutschland. Zweite Hälfte, Erste Lieferung, pag. 98, 99.

Cythere Bosquet, 1850. Description des Entomostracés fossiles des terrains tertiaires de la France et de la Belgique. pag. 49 et suivantes.

Cypridinae spec. Reuss, 1851. Naturwissenschäftl. Abhandl. von WuH. Haidinger. Vierter Band, $1^{\text {ste }}$ Abth. pag. 49, 50.

Caractères Génériques. - La carapace des Cythere est formée de deux valves inégales à contour oblong, ellipsoïdal, ovale, rhomboïdal ou sub-tétragonal, de consistance corneocalcaire, réunies sur leur bord dorsal ou supérieur par une charnière garnie de dents plus ou moins fortes suivant les espèces, mais constamment en nombre défini. Ces valves peuvent s'entrouvrir librement du côté pectoral, et présentent vers le milieu, et un peu en avant de la moitié de leur longueur, un tubercule plus ou moins apparent selon les espèces, et dont la place est accusée à l'intérieur de chaque valve par une fossette ovale ou arrondie. La valve gauche est constamment un peu plus grande que la valve droite et embrasse tant soit peu toute la circonférence du bord de celle-ci.

Le bord dorsal interne de chaque valve présente une partie élevée ou barre longitudinale, à côté de laquelle on remarque un sillon ou partie déprimée. Sur la valve droite, la partie externe du bord est plus haute que l'interne; tandis que sur la valve gauche c'est tout le contraire qu'on observe. La barre longitudinale, surtout dans la valve gauche, est étroite, arrondie et polie. Lors de la réunion des deux valves, la partie externe plus haute du bord dorsal de la valve droite, vient se placer au dessus de la partie externe plus déprimée du,bord de la valve gauche, tandis que la partie interne plus basse du bord de la valve droite, reçoit la partie interne plus haute, ou plutôt la barre cardinale de la valve gauche.

Sur la valve droite, la charnière est formée de deux dents, l'une antérieure et l'autre postérieure, qui sont insérées sur la partie interne déprimée du bord, et qui sont reçues dans deux fossettes de la valve opposée. Sur la valve gauche il y a constamment deux dents antérieures et quelquefois une très-petite dent postérieure rudimentaire et le plus souvent presque nulle. Des deux dents antérieures de cette valve, l'une est placée en avant de la grande fossette et l'autre immédiatement en arrière de cette fossette sur l'extrémité antérieure de la barre 
cardinale; tandis que la petite dent cardinale postérieure, quand elle existe, est située sur l'extrémité postérieure de la barre, immédiatement en avant de la fossette postérieure. La dent cardinale antérieure de la valve droite est plus ou moins comprimée, ou plus ou moins conoïdale suivant les espèces, et toujours plus grande que la dent cardinale postérieure; elle est passablement épaisse à la base et plus ou moins pointue à son extrémité libre. Les deux dents cardinales de la valve droite sont constamment inclinées en dehors, tandis que celles de la valve gauche sont droites, ou faiblement inclinées vers le centre des valves.

Les deux fossettes cardinales de la valve gauche sont plus ou moins profondes, suivant que les dents qu'elles servent à recevoir sont plus ou moins longues. Sur la valve droite les fossettes sont très-peu sensibles; celle qui est située immédiatement en arrière de la dent cardinale antérieure, est en général la seule qui soit passablement bien prononcée.

Le bord pectoral de chaque valve offre ordinairement, vers le milieu, une partie infléchie plis ou moins prononcée selon les espèces, et qui, sur les carapaces fermées, se fait déja remarquer au dehors par une sorte de petit sinus, ou de petite Lunule. Ché les espèces qui présentent un rebord marginal externe, cette Lunule devient le plus souvent très-apparente, parceque le rebord forme alors une saillie sémilunaire mince, assez sensiblement projetée en dehors et produite par un accroissement local de sa partie externe ou libre. C'est à la partie infléchie que je viens de mentionner, à côté de laquelle le bord valvaire est le plus mince et le plus aigu, que Mr. Convues a donné le nom de lame pectorale. Lors de la réunion des deux valves, la lame pectorale de la valve droite vient se placer en dedans sur celle de la valve gauche, dans une cavité peu profonde, qui est destinée à sa réception.

Sur le bord interne de la valve droite, on remarque deux sillons étroits, qui ont leur origine à chaque extrémité de la lame pectorale; l'un de ces sillons se dirige en avant, devient de plus en plus étroit sur le large bord antérieur, et va se terminer à côté de la dent cardinale antérieure; l'autre sillon au contraire, se dirige en arrière jusqu'à l'extrémité postérieure, oû il disparait après être devenu plus étroit et moins profond. Ces deux sillons correspondent à une partie saillante du bord interne de la valve gauche.

Quand on examine les valves des Cythere en dehors, on remarque qu'elles sont ordinairement arrondies en avant, et plus larges dans leur moitié antérieure; tandis qu'en arrière, où elles sont habituellement plus étroites, elles se terminent assez souvent par une partie comprimée, ou par une pointe plus ou moins aiguë, qui s'écarte ordinairement de leur axe longitudinal, en se portant généralement vers le côté pectoral, ou, ce qui n’arrive que très-rarement, vers le côté dorsal.

Les bords extérieurs des valres de la plupart des Cythere sont épaissis, principalement 
le long du côté antérieur. Il arrive assez souvent aussi, que le bord supérieur et même tout le bord des valves est épaissi, ou plutôt marginé d'un rebord, comme par exemple, chez mes Cythere formosa, lichenophora, cristata et horridula. Les deux extrémités, principalement la postérieure, sont le plus souvent assez fortement comprimées, et c'est ordinairement près de l'extrémité postérieure, ou immédiatement en avant de la partie comprimée postérieure, que se trouve la partie la plus haute de la voûte dorsale des deux valves. De ce point le dos des valves descend vers le bord antérieur, ou la partie comprimée antérieure, par une pente plus ou moins douce; il se rattache le plus souvent également au bord supérieur par une pente plus ou moins lente, tandis qu'il rejoint le bord pectoral par une pente très-rapide, ou même qu'il y retombe perpendiculairement. Dans ce dernier cas, la région pectorale du bouclier entier présente assez souvent une surface passablement large, plane, ou même un peu excavée, dont le contour est fréquemment cordiforme, triangulaire, hasté ou sagittiforme. Cette surface est divisée longitudinalement en deux parties égales par le rebord plus ou moins saillant du bord valvaire pectoral, et est séparée de la voûte dorsale des valves par une arête ou une carène plus ou moins aiguë.

Aux angles antérieur et postérieur du côté dorsal, le bord valvaire est plus ou moins fortement projété en dehors suivant les espèces, et le plus souvent en raison directe du développement des dents cardinales. La projection de l'extrémité antérieure dn bord dorsal est toujours la plus forte; elle forme assez souvent une proéminence sémicirculaire ou un appendice en forme d'oreillette, sur lequel on observe constamment un très-petit tubercule arrondi, le plus souvent luisant comme du verre, et dont la place correspond exactement à celle qu'occupe à l'intérieur la dent cardinale antérieure. Autour de ce petit tubercule, le bord valvaire est plus ou moins épaissi, sans doûte pour donner à la pression des dents, proportionnellement grandes et fortes, un point d'appui aussi solide que cela était possible, eu égard au peu d'épaisseur des valves. J'ai désigné cette partie des valves sous le nom d'oreillette cardinale antérieure. L'angle cardinal postérieur produit aussi, chez la plupart des espèces, une saillie marginale sémicirculaire en forme d'oreillette, constamment plus petite que l'antérieure, mais très-sensible en cet endroit, parceque immédiatement en arrière de la place qu'elle occupe, il y a une partie du bord valvaire qui est fort mince.

Ces deux oreillettes cardinales manquent totalement à toutes les espèces des genres Cytherella, Bairdia, Candona et Cypris, et fournissent par conséquent un caractère certain pour reconnaître les Cythere, même dans les cas oú il n'est pas possible d'examiner l'intérieur et la charnière. J'ai désigné sous le nom de région dorsale, l'espace compris, quand on examine le bouclier du côté superieur, entre les deux oreillettes cardinales. 
Un peu en avant de la partie moyenne de chaque valve des Cythere, et constamment dans leur axe longitudinal, on observe un autre tubercule, de forme et de grandeur très-variées. Ce tubercule, qui est bien prononcé et très-proéminent chez quelques espèces, est peu apparent, presque confondu avec le reste de la surface, et alors presque imperceptible dans d'autres. A l'intérieur des valves au contraire, la place de ce tubercule est toujours indiquée par un enfoncement ovale ou arrondi. J'ai donné à ce tubercule le nom de tubercule sub-central, afin de le distinguer du tubercule oculaire des Cypridina.

L'animal des Cythere n'a qu'un seul oeil conique; il a deux antennes cylindriques, composées de cinq articles sétifères, et deux antennes pédiformes qui, au lieu d'être pourvues d'un paquet dé soies, comme celles des Cypris, possèdent un filament articulé assez fort; il a trois paires de pattes grèles et cylindriques, qui paraissent toutes au dehors de la carapace et dont la paire postérieure est plus longue que les deux autres. Les Cythere sont marines, rampent au fond de la mer, ou grimpent sur les Fucus ou les Flustres et ne sont probablement que littorales.

Le genre Cythere, dont les Naturalistes n'ont fait connoître jusqu'à présent qu'un nombre fort restreint d'espèces vivantes, a de nombreux représentants fossiles. Les terrains Paléozoiques paraissent n'en renfermer que très-peu d'espèces; d'après Mr. Jones, il n'en existe que quatre à cinq dans le lias et dans les terrains Oölitiques; j'en connais environ cent-quatorze dans les divers étages du terrain tertiaire; vingt-trois autres ont été signalées par divers Paléontologistes dans les Systèmes moyen et supérieur de la formation crétacée. Parmi ces vingt-deux espèces crétacées il y en a treize qui proviennent des couches crayeuses des environs de Maestricht, et qui ont déja été décrites par moi en 1847. A ces treize espèces, viennent se joindre trentequatre nouvelles, découvertes après des recherches assidues dans plusieurs Systèmes du terrain crétacé, qui a un si grand développement dans le Duché de Limbourg.

1. GYTHERE FUSIFORMIS BOSQUET, 1853.

Pl. VIII, fig. $6 a, b, c, d$.

Cypridina fusiformis Bosauet, 1847. Description des Entomostracés fossiles de la craie de Maestricht. pag. 11, pl. I, fig. $4 a-f$.

"

Bosquet, 1847. Mémoires de la Sociéte royale des Sciences de Liége. pag. 361 , pl. I, fig. 4 a $a$.

Cythereis "J Jones, 1849. A Monograph of the Entomostraca of the Cretaceous formation of England. Appendix pag. 36. 
Valves lisses, allongées-fusiformes, plus de deux fois aussi longues que larges, arrondies en avant et terminées en arrière en une pointe émoussée. Leur voûte dorsale est le plus convexe au milieu, et de ce point elle se rattache aux deux extrémités par une pente assez douce. $\mathrm{La}$ carapace présente une section transversale à contour ovale, et une section longitudinale à contour rhomboidal.

Dimensions. - Longueur 0,8 de millim., hauteur 0,4 de millim. et épaisseur 0,35 de millim.

Rapports et Différences. - Cette espèce se reconnait aisement à ses valves lisses et allongées en forme de fuseau.

Gisement et Localités. - Elle est assez rare et se trouve dans le Système Maestrichtien à St. Pierre, à Keer, à Nedercanne, à Bemelen, à Geulhem et à Fauquemont, dans le Duché de Limbourg. En Belgique, elle a été trouvée à Sichen.

2. CYTHERE FAVROdXANA BosQUeT, 1853.

PI. VIII, fig. $7 a, b, c, a$.

Cypridina Favrodiana Bosodet, 1847. Descript. des Entomostr. foss. de la craie de MIaestricht. pag. 11, pl. I, fig. $5 a-d$.

" "Bosquet, 1847. Mémoires de la Société Royale des Sciences de Liége. pag. 361 , pl. I, fig. $5 a-d$.

Cythereis "J Jones, 1849. A Wonograph of the Entomostr. of the cretaceous form. of England. Appendix pag. 36.

Carapace formée de deux valves épaisses, très-fortement bombées, à surface lisse. Ces valves sont oblongues-sub-tétragonales, obliquement arrondies en avant et terminées en arrière par une partie comprimée obtuse, en forme de bec et dirigée vers le côté inférieur. Le bord pectoral est légèrement sinué vers le tiers antérieur de sa longueur; le dorsal offre une oreillette cardinale antérieure peu prononcée. La dent cardinale antérieure de la valve droite est conoidale, la postérieure sub-quadrangulaire.

Le bouclier présente, vers le tiers postérieur de sa longueur, une section transversale à contour ovale-cordiforme.

Dimensions. - Longueur 1,1 de millim., hauteur 0,7 de millim. et épaisseur 0,6 de millim.

Iapports et Difrérences. - Cette Cythere avoisine la précédente; mais elle en diffère tellement par la trille, par l'épaisseur et la forme de ses valves, qui sont fortement bombées dans toute leur longueur, qu'on peut facilement la distinguer au premier abord.

Gisement et Localités. - Elle est peu commune dans le Système Maestrichtien à Neder- 
canne, à St. Pierre, à Bemelen, à Geulhem et à Fauquemont; ainsi que dans le calcaire grossier à silex gris (Systéme Sénonien Dumont) à St. Pierre.

3. Cythere CONCEntrica Reuss, spec. 1846.

PI. VIII, fig. $8 a, b, c, d$.

Cytherina concentrica Reuss, 1846. Versteinerungen der Bölmischen Kreide-formation. Zweite Abth. pag. 105, pl. XXIV, fig. $22 a, b, c$.

Cythere sculpta Connoer, 1846. Mémoires de la Soc. Géologique de France. $2^{\text {me }}$ série, tome $1^{\text {ier }}, 2^{\text {me }}$ partie, pag. 201, pl. VIII, fig. 20-23.

Cytherina concentrica WiLLianson, 1847. Transactions of the Manchester Literary and Plilosophical Society. Vol. VIII. - Mémoir on some, etc. pag. 82, pl. IV, fig. 77.

Cypridina Roemeriana Bosouet, 1847. Descr. des Entom. foss. de la craie de Maestricht. Pl. 12, 13, pl. II, fig. $2 a-f$.

Bosquet, 1847. Ménoires de la Soc. royale des Sciences de Liege. tome IV, pp. 262, 263, pl. II, fig. $2 a-f$.

Cythere punctatula Jones, 1849. A MTonograph of the Entomostraca of the cretaceous formation of England. pag. 11, pl. I, fig. $2 a-n$ (non RoEMER).

" "Bosquet, 1850. Descript. des Entomostr. fossiles du terrain tertiaire de la France et de la Belgique. pp. 73-75 (non Roeuer).

Cypridina concentrica Reuss apud Geinitz, 1850. Das Quadersandstein-gebirge oder Kreide-gebirge in Deutschland. pag. 98.

Var. A. vingineA Jones. Valvis sub-laevibus, vel plicis, seu foveolis obsoletis.

Cypridina Althi Reuss, 1851. Naturwissenschäfliche Abhandlungen von WilH. Haidivger, Vierter Band, $1^{\text {ste }}$ Abth., pag. 49, tab. VI, fig. $10 a-c$.

Var. B. SUBCARINATA Bose. 1853. Valvis carinatis vel sub-carinatis, plicis concentricis plus minusve obsoletis, dente postico unico. Pl. VIII, fig. $8^{\prime} a, b, c, d$.

Valves ovales. sub-Ihomboïdales, arrondies aux deux extrémités et marginées d'un rebord comprimé très-étroit. Leur bord supérieur est fortement arqué et l'inférieur est comprimé et presque droit. Ces valves sont tellement enflées au côté pectoral, que leur voûte dorsale dépasse de beaucoup le bord, et que ce côté parait très-fortement arqué. La surface, qui est le plus souvent recouverte de bourrelets ou de plis concentriques et, vers le milieu, de granulations, est parfois réticulée dans les jeunes individus, et les réticulations sont alors arrangées concentrique- 
ment. Selon MIr. Jones, les plis ou les parties élevées du réseau sont armées de petites épines et en s'épaississant recouvrent le réseau, jusqu'à ce que apparaissent plus tard les points creux très-petits et plus ou moins concentriques. Dans les individus adultes on ne voit plus que les côtes ou gros plis, qui sont fortement marqués et réguliers sur la partie pectorale des valves, qui deviennent plus fins vers la partie supérieure, et qui, vers la partie centrale des valves, sont divisés en des corrugations ou granulations irrégulières. La partie comprimée postérieure est garnie, dans les échantillons parfaiteinent adultes, de trois petites dents, qui paraissent toujours manquer dans la variété A (Virginea de Mr. Jones), et dont il ne reste le plus souvent qu'une seule, dans ma variété $B$ (subcarinata).

Quelquefois les valves ont conservé des restes de leurs couleurs, et sont alors d'un brunrougeàtre, excepté vers la partie centrale, qui est blanche.

Dimensions. - Longueur 0,5 de millim., hauteur 0,45 de millim. et épaisseur 0,55 de millim.

Observations. - Après avoir examiné avec soin les deux carapaces, provenant du Hilsthon, qui ont servi à Mr. Fr. Ad. Roemer pour l'établissement de sa Cy there puuctatula, je crois pouvoir affirmer que cette espèce n'est pas la même que la Cythere concentrica Reuss, comme Mr. R. Joves l'avait cru. Je ne doute presque plus, que les deux échantillons de la collection de Mr. Roemer, ne soient de jeunes individus de sa Cythere Hilseana, trouvée dans la même localité.

Je dois à l'obligeance de Mr. Connuel de Wassy (Haute-Marne) plusieurs échantillons de l'esjèce qui a été décrite par cet auteur sous le nom de Cythere amygdaloïdes. Cette dernière ne me parait différer aucunement de la Cythere Hilsean'a Roemer. - A côté de ces échantillons du terrain Néocomien de Wassy et sur les mêmes plaquettes (comme les appelle Mr. Connuru), je remarque une infinité de valves et de carapaces de toute grandeur, qui me paraissent presque toutes être de jemmes ages de la Cythere Hilseana, et parmi lesquelles j'en vois un assez grand nombre, qui sont parfaitement identiques à celles de la Cytherina punctatula RoEuer.

Gisement et Localités. - Cette Cythere se rencontre fréquemment dans le Système Maestrichtien à Nedercanne, à St. Pierre, à Gronsveld, à Bemelen, à Geulhem, à Fauquemont et à Kunraede et dans une couche qui parait appartenir aux parties inférieures du même Système à Schin-op-Geul; dans le calcaire grossier à silex gris (Système Sénonien Duxont) entre St. Pierre et Petit-Lanaye, et dans le psammite glauconifère (Système Hervien Dumont) à Simpelveld et entre Vaels et Gimenich. En Belgique elle se trouve dans le Système Maestrichtien de Sichen, de Fol $\mathrm{\text {-les}}$-Caves et de Ciply près Mons, ${ }^{1}$ ) dans cette dernière localité

1) Des échantillons du terrain Maestrichtien et Sénonien de cette localité de Belgique m’ont été envoyés par Mr. De Koninck, professeur de Chimie et de Paléontologie à l'Université de Liège. 
elle se trouve aussi dans le Système Sénonien (calcaire grossier à silex gris). En Suède, elle se rencontre dans des couches contemporaines de celles du Système Maestrichtien, près d'Ifö (Schoonen). D'après Mr. Jones, elle se trouve dans le Greensand de Warminster, dans le gault de Folkstone et de Leacon-Hill, dans le Chalk-marl de Douvres, dans le detritus de Charing et dans la craie blanche du Sud-Est de l'Angleterre. Suivant Mr. Reuss, elle est rare dans le plänermergel de Luschitz et de Rannay en Bohème, et de Lemberg en Gallicie, et selon Mr. Connuer, elle est abondante dans l'étage Neocomien de Wassy, département de la Haute-Narne, en France.

4. CYTHERE FURGIFERA Bosquet, 1853.

Pl. VIII, fig. $9 a, b, c, d$.

Cypridina furcifera Bosquet, 1847. Description des Entomostracés fossiles de la craie de Maestricht. pp. 13, 14, pl. II, fig. $3 a-d$.

Bosquet, 1847. Mémoires de la Société royale des Sciences de Liége.

Tom. IV, pp. 363, 364, pl. II, fig. $3 a-d$.

Cythereis "Jones, 1849. A Monograph of the Entomostraca of the cretaceous formation of England. Appendix pag. 36.

Cette espèce a des valves oblongues-sub-rhomboïdales, tellement enflées au côté pectoral, que leur voûte dorsale dépasse de beaucoup le bord, et que ce côté parait fortement arqué Leur bord supérieur est arqué et les deux oreillettes cardinales font, aux extrémités de ce bord, une saillie assez forte. Le bord antérieur et le lobe comprimé postérieur, sont obliquement arrondis. Le premier est marginé d'un rebord comprimé lisse et le dernier est gami de quatre à cinq dents, inégales et acuminées. La surface est lisse et luisante, et offre trois côtes inégales, dont la médiane se bifurque vers la partie moyenne de chaque valve.

Le bouclier vu par l'extrémité antérieure a un aspect cordiforme.

Quelquefois les valves ont conservé des traces de leurs couleurs, ct les parties comprimées des extrémités sont alors d'un jaune-foncé ou d'un jaune-orangé, tandis que le reste de la surface est d'un blanc de lait.

Dimensions. - Elle a 0,95 de millimètre de longueur et 0,6 de millimétre d'épaisseux.

Rapports et Dimérences. - Cette espèce se distingue aisement de la précédente, par une taille plus grande, par son lobe comprimé postérieur assez large, par les ornements de sa surface et par ses oreillettes cardinales.

Gisement et Localités. - Elle est peu commune dans le Système Maestrichtien à St. 
Pierre, à Nedercanne, à Gronsveld, à Bemelen, à Geulhem, à Fauquemont et à Kunraede. En Belgique elle se trouve dans le Système Naestrichtien à Petit-Lanaye, à Wonck et à Sichen.

う. GYTHERE EUGLYPHA nov. spec.

Pl. $V$, fig. $7 a, b, c, d$.

Valves épaisses, assez solides, fortement enflées, obliquement arrondies aux deux extrémités, et ayant les bords pectoral et dorsal arqués. Le dernier offre à clacune de ses extrémités une oreillette cardinale très-saillante. La surface présente sur son tiers médian plusieurs sillons longitudinaux très-étroits, qui séparent des côtes déprimées, inégales en longueur, et parmi lesquelles une des médianes se bifurque vers la moitié de sa longueur. Les parties antérieure et postérieure de la surface sont ornées d'un grand nombre de tubercules arrondis et peu saillants. Oûtre ces petits tubercules on en remarque quelques-uns, qui sont à peu près deux fois aussi gros, le long de la moitié inférieure du bord antérieur.

La carapace présente une section transversale à contour arrondi-hexagonal, et une section longitudinale (à l'endroit où les valves sont le plus enflées) à contour ovale-piriforme.

EBimensions. - Longueur 1 millim., hauteur 0,6 de millim. et épaisseur 0,55 de millim.

IRapports et idiférences. - La Cythere euglypha se rapproche des deux espèces précédentes. Elle se distingue cependant de la concentrica, non seulement par les omements de sa surface, mais aussi par ses dimensions, par ses valves épaisses, par sa région dorsale large et par ses oreillettes cardinales saillantes; elle s'eloigne de la Cythere furcifera, par ses valves épaisses, plus larges, sans dentelures au lobe comprimé postérieur et par les ornements de la surface.

Gisement et Localités. - Cette espèce se rencontre dans les couches à Bryozoaires du Système Maestrichtien à Nedercanne, à St. Pierre, à Gronsveld, à Bemelen, à Geulhem et à Fauquemont.

6. cythere internupta Bosquet, 1853 (non Cythereis interrupta Jones).

PI. VIII, fig. $10 a, b, c, d$.

Cypridina interrupta Bosavet, 1847. Description des Entomostracés fossiles de la craie de Naestricht. pag. 12, pl. II, fig. $4 a-d$.

Bosquet, 1847. Mémoires de la Société royale des Sciences de Liége. tom. IV, pag. 362, pl. II, fig. $1 a-d$.

Cythereis "Jones, 1849. A Monoyraph of the Entomostraca of the cretaceous form. of England. Appendix pag. 36 (non pag. 16). 
Les valves de cette Cythere sont allongées-ellipsoidales, un peu plus larges en avant qu'en arrière, elles sont obliquement arrondies aux deux extrémités; leurs bords supérieur et inférieur sont presque parallèles et faiblement sinués au milieu; leur voûte dorsale, qui est déprimée au milieu, se rattache aux deux extrémités et au bord supérieur par une pente rapide, et retombe perpendiculairement sur le bord inférieur; elle est divisée en trois grandes côtes, par deux sillons larges, mais peu profonds; toute sa surface est creusée en outre d'un grand nombre de sillons concentriques tres peu profonds, au fond desquels on remarque des lignes creuses assez régulièrement interrompues. Le tubercule sub-central est situé sur la partie antérieure de la grande côte médiane. Ce tubercule est ordinairement lisse et peu apparent; mais dans les individus qui ont conservé des restes de leur couleur, sa place est indiquée par une tache blanche et luisante, contrastant avec le reste de la surface, qui est d'un jaune foncé ou d'un brun-rougeâtre.

Le bouclier entier, vu pạr l'extrémité antérieure, présente un aspect trigonal sub-sexlobé, vu par le côté supérieur ou inférieur, il offre un aspect allongé et irrégulièrement hexagonal.

Bimensions. - Longueur 0,9 de millimètre, hauteur 0,5 de millimètre et épaisseur 0,45 de millimètre.

IRapports et Différences. - Mr. Jones a décrit et m’a communiqué une espèce de la formation crétacée de l'Angleterre, qu’il a cru être la même que celle qui nous occupe ici. L'espèce Anglaise est tout-à-fait différente de la nôtre; elle se distingue par une taille toujours plus petite, par la voûte dorsale de ses valves, offront vers le quart postérieur de sa longueur, au côté pectoral, une grosse pointe obtuse, par les points creux de sa surface, beaucoup plus petits et non allongés, ${ }^{1}$ ) et surtout par le manque des deux larges sillons longitudinaux.

Gisement et Localités. - Cette Cythere se trouve en assez grande abondance dans le Système Maestrichtien à St. Pierre, à Kunrade et à Schin-op-Geul, et dans le calcaire grossier à silex gris, entre St. Pierre et Petit Lanaye, dans le Duché de Limbourg. Elle est assez fréquente aussi en Belgique, dans le Système Maestrichtien à Sichen et à Wonck et dans le calcaire grossier à silex gris, à Ciply près Mons. Je viens de la découvrir également dans le terrain crétacé supérieur d'Ifö $\left.{ }^{2}\right)$ (Schoonen) en Suède.

1) Ces points, dans ma Cythere interrupta, sont semblables à des piqûres, qu'on produirait au moyen de la pointe d'un canif.

2) Je dois la communication de plusieurs fossiles, ainsi que d'un échantillon de terrain erétacé de cette localité, à l'obligennce de Mr. le Dr. Fr. von Hagexow de Greifstrald. 
7. cythere pulchella Bosquet, 1853.

Pl. IX, fig. $1, a, b, c, d$.

Cypridina pulchella Bosquet, 1847. Description des Entomostraces fossiles de la craie de Maestricht. pp. 14, 15, pl. II, fig. $5 a-d$.

Bosodet, 1846. Mémoires de la Société royale des Sciences de Liége. tom. IV, pp. 364,365, pl. II, fig. $5 a-d$.

Reuss apud GeInitz, 1850. Das Quadersandstein-gebirge oder Kreidegebirge in Deutschland. Erste Lieferung pag. 98.

Cythereis "Jones, 1849. A Monograph of the Entomostraca of the cretaceous form. of England. Appendix. pag. 36.

Var. B. Superficie nitida, sulcis longitudinalibus non punctatis, extremitatibus edentulis. Bosqur T. pl. IX, fig. $2 a-d$. Cypridina Foersteriana Bosquet, 1847. Description des Entomostracés fossiles de la craie de Maestricht. pag. 14, pl. II, fig. $4 a-d$.

Bosquet 1847. Mémoires de la Société royale des Sciences de Liége. tom. IV, pag. 364 , pl. II, fig. $4 a-d$.

Cythereis "Jones, 1849. A Monograph of the Entomostraca of the cretaceous formation of England. Appendix pag. 36.

Carapace formée de deux valves oblongues, rhomboïdales; marginées en avant d'un rebord comprimé, dont la surface est ornée de quelques points enfoncés, oblongs et rayonnants, et dont le bord 'est garni de 4-5 dentelures courtes; ces valves se terminent en arrière par un lobe comprimé sub-trigone, muni de 3-1 dents aiguës. La partie voûtée de ces valves se rattache par une pente assez douce aux bords supérieur et antéricur, et retombe perpendiculairement sur le bord inférieur et sur le lobe comprimé postérieur; la surface est divisée longitudinalement en trois côtes inégales, par deux sillons assez profonds et ponctués sur leurs bords; de ces trois côtes, la plus saillante et la plus large est celle qui se trouve le long du côté pectoral; elle porte postérieurement une dent aiguë, et sa surface est creusée de deux lignes flexueuses. Les deux oreillettes cardinales forment des saillies assez fortes. Le bouclier présente une section transversale à contour sub-trigone et une section longitudinale à contour piriforme.

Les échantillons qui ont conservé des restes de leur couleur, ont les parties comprimées des deux extrémités d'un jaune-orangé ou d'un brun-foncé.

Les valves de la variété $B$, différent de celles du type de l'espèce par le manque de dentelures aux extrémités et de points creux dans les deux sillons longitudinaux et sur la surface 
de la partie comprimée antérieure. Ce manque d'ornements parait dépendre uniquement de l'âge des individus qui ont produit ces valves. Il me semble que ce ne sont que des valves de vieux individus.

Dimensions. - Longueur 0,8 de millim., hauteur 0,45 de millim. et épaisseur 0,5 de millim.

Rapports et Biférences. - Elle se rapproche des Cythere elegans Bosquer et triplicata Roemer. ${ }^{1}$ ) Elle se distingue néanmoins au premier abord de la première, par ses valves plus larges, à oreillette cardinale antérieure beaucoup plus grande, par les deux excavations longitudinales de sa surface non creusées de sillons transversaux, et par son lobe comprimé postérieur sub-trigone. Elle diffère de la Cythere triplicata, par sa taille constamment plus petite, par ses valves beancoup moins larges en avant, à limbe marginal antérieur plus étroit et à oreillette cardinale antérieure beaucoup moins saillante. Les trois côtes longitudinales de sa partie voûtée sont arrondies, tandis que celles de l'échantillon qui a été figuré par Mr. Fr. AD. Roexer, et que j'ai eu occasion d'examiner avec soin, étaient anguleuses au milieu. Je doute que la Cythere auriculata, dont je dois des échantillons à l'obligeance de l'auteur qui l'a décrite, soit bien la même que la Cythere triplicata, comme l'a cru Mr. Jones.

Gisement et Localités. - Cette Cythere se rencontre dans le Système Maestrichtien à St. Pierre, Bemelen, Gronsveld et Fauquemont, dans le calcaire grossier à silex gris entre St. Pierre et Petit Lanaye, dans le Système Hervien? entre Benzenrathof et Keverberghof près Heerlen, et dans le psammite glauconifère (Système Hervien) à Slenaken.

8. CYTHERE STRIATO-Costata nov. spec. 1853.

Pl. V, fig. $8 a, b, c, d$.

Valves allongées-ellipsoïdales; à bords pectoral et dorsal faiblement arqués; à bord antérieur obliquement sémi-circulaire et marginé d'un limbe comprimé, qui se termine brusquement à l'extrémité antérieure du bord dorsal, en donnant naissance à une oreillette assez saillante; terminées en arrière par un lobe comprimé arrondi et bidenté. Leur partie voûtée est fortement enflée au côté pectoral; elle est divisée en trois côtes longitudinales, de largeur inégale, par deux sillons larges, mais peu profonds, et faiblement coudés au milieu. La surface des trois côtes longitudinales, ainsi que celle du rebord compriné antérieur, est creusée de quelques lignes très-fines. La région dorsale est aplatie et sillonnée transversalement.

Le , bouclier vu par l'extrémité antérieure, présente un aspect cordiforme sub-trigone; vu en dessus ou en dessous il offre un aspect ovale-sub-piriforme.

Dimensions. - Longueur 0,7 de millim., hauteur 0,4 de millim. et épaisseur 0,3 de millim.

1) Fr. Ad. Rosmek, 1840. Versteinerungen des Nord-deutschen Kreide-gebirges. pag. 104, pl. XVI, fig. 16. 
Rapports et Difrérences. - Cette espèce est constamment plus large aux extrémités, et a la voûte dorsale toujours plus bombée que la précédente; les deux sillons longitudinaux qui séparent les côtes de sa surface ne sont jamais creusés de points.

Gisement et Localités. - Elle est assez rare et n’a encore été rencontrée que dans les couches à Bryozoaires du Système Maestrichtien à Bemelen, à Geulhem et à Fauquemont.

9. CYThere propingua nov, spec, 1853.

Pl. V, fig. $9 a, b, c, d$.

Cette espèce a des valves déprimées, allongées sub-pentagonales, obliquement arrondies aux deux extrémités; marginées en avant d'un rebord comprimé bi-ou tri-sillonné; terminées en arrière par un lobe comprimé assez étroit et quadridenté; leur partie voûtée se rattache au bord dorsal et à la partie comprimée antérieure par une pente assez douce; elle rejoint le lobe comprimé postérieur et le bord pectoral par une pente très-rapide. La partie voûtée offre deux dépressions longitudinales flexueuses assez larges, dont l'une sépare deux côtes presque droites et lisses. La surface de chacune de ces côtes est creusée de deux sillons. La région dorsale est équilatérale dans toute sa longueur, elle est creusée de dix sillons obliques, dont cinq à chaque côté de la ligne de jonction des valves, et dont les quatre antérieurs sont opposés en croix.

La carapace, vue par l'extrémité antérieure, présente un aspect sub-deltoïde; vue en dessus ou en dessous elle offre un aspect sub-piriforme-allongé.

mimensions. - Longueur 0,8 de millim., hauteur 0,4 de millim. et épaisseur 0,32 de millim.

Rapports et mifrérences. - Elle se rapproche un peu de la précédente, mais s'en distingue facilement par son oreillette cardinale antérieure non saillante, par son lobe comprimé postérieur plus étroit et garni de quatre dents, et surtout par la côte inférieure de sa voûte dorsale, réunie en avant à la partie comprimée antérieure.

Gisement et Localités. - Elle est très-rare et n’a été trouvée qu’à Bemelen et à Fauquemont.

10. Cythere elegans Bosquet, 1853.

Pl. IX, fig. $3 a, b, c, d$.

Cypridina elegans Boseuet, 1847. Description des Entomostracés fossiles de la craie de Maestricht. pp. 15, 16, pl. III, fig. $1 a-d$.

" "Bosquet, 1847. Mémoires de la Société royale des Sciences de Liége. tom. IV, pp. 365, 366, pl. III, fig. $1 a-d$.

Cythereis "J Jones, 1849. A Monograph of the Entomostraca of the cretaceous formation of England. Appendix pag. 36. 
Les valves de cette Cythere présentent un contour sub-ovale-allongé; elles sont arrondies en avant et marginées d'un rebord comprimé, obscurement ponctué, et garni de 8-9 dentelures courtes et aiguës; elles sont terminées en arrière par un lobe comprimé d'une forme tétragonale et garni de quatre dents obtuses, un peu plus grandes que celles du bord antérieur. La partie voûtée est ¿très-enflée vers le côté pectoral, et sa surface est divisée en trois côtes longitudinales lisses, par deux sillons assez profonds, dont l'un est droit et creusé de points nombreux, tandis que l'autre est faiblement arqué et orné, dans sa moitié postérieure, de rides transversales. Deux de ces côtes longitudinales portent en arrière une épine aiguë.

Le bouclier offre vers le milieu de sa longueur une section transversale à contour deltoidequinquelobé; vu du côté pectoral, il présente un aspect sub-sagitté piriforme.

Quand la carapace a conservé des restes de ses couleurs, la partie voûtée est blanche et les deux parties comprimées des extrémités sont d'un orangé foncé.

minensions. - Longueur 0,9 de millimètre, hauteur et épaisseur 0,5 de millimètre.

Rapports et Différences. - Elle se rapproche de la Cythere pulchella par ses valves tricostées, mais s'en distingue nettement par sa forme, par son lobe comprimé postérieur, et par les sillons transversaux dans les dépressions qui séparent les côtes de sa surface.

Giscment et Localités. - Cette espèce est peu commune dans le Système Maestrichtien à Gronsveld, à St. Pierre, à Keer, à Bemelen, à Geulhem, et à Fauquemont; elle est rare dans ce même Système à Kunraede, ainsi qu’à Schin-op-Geul et à Bulkomsdal, dans le Duché de Limbourg. Elle n'est pas rare dans le Système Maestrichtien à Sichen, et très-rare à Jandrain, en Belgique.

\section{GYThere radiosa nov. spec. 1853.}

Pl. V, fig. $10 a, b, c, d$.

Valves allongées sub-tétragonales, arrondies en avant et marginées d'un limbe comprimé lisse; terminées en arrière par un lobe comprimé assez large, muni de quatre dents courtes et obtuses; à bords supérieur et inférieur droits et faiblement divergents en avant. Leur partie voûtée se rattache aux bords antérieur et supérieur par une pente assez douce, elle rejoint le bord inférieur par une pente très-rapide et retombe presque perpendiculairement sur le lobe comprimé postérieur; sa surface lisse présente un tubercule sub-central obliquement arrondi, et en arrière de celui-ci, quatre sillons rayonnants vers le lobe comprimé postérieur et vers le bord supérieur.

La carapace offre une section transversale à contour arrondi-sub-deltoide et une section longitudinale à contour cunéiforme. 
Dimensions. - Longueur 0,75 de millim., hauteur 0,35 de millim. et épaisseur 0,3 de millim.

Gisement et Localités. - Cette Cythere est extrémement rare et n’a encore été trouvée jusqu’ici que dans les couches ì Bryozoaires du Système Maestrichtien ì Bemelen et près Nedercamne.

12. CYTHERE SUBTETRAgona nov. spec. 1853.

Pl. V, fig. $11 a, b, c, d$.

Cette Cythere a des valves allongées, dont le contour est tétragonal; leurs bords inférieur et supérieur sont presque droits et parallèles, le dernier est faiblement sinueux par la saillie qu'y forment les petites oreillettes cardinales postérieure et antérieure. En avant de cette dernière on remarque une petite échancrure triangulaire; le bord antérieur est sub-sémicirculaire et marginé d'un limbe comprimé, dont la surface est creusée de plusieurs lignes rapprocliées et parallèles au bord; la partic comprimée postérieure est plus étroite que l'antérieure et obliquement tronquée. La partie voûtée se rattache aux bords antérieur et supérieur par une pente assez rapide et retombe perpendiculairement sur le bord pectoral et sur le lobe comprimé postérieur. La surface du dos des valves est divisée en trois côtes de largeur inégale, par deux sillons longitudinaux, dont l'un est creusé d'un grand nombre de sillons transversaux. Des trois côtes longitudinales, celle qui longe le côté inférieur est la plus large et la plus haute, et sa surface est marquée de plusieurs lignes longitudinales.

La région dorsale est presque linéaire et lisse.

Le bouclier, vu par l'éxtrémité antérieure, présente un aspect deltoide; vu du côté pectoral, il offre un aspect cunéiforme sub-cordé.

Dimensions. - Longueur 0,75 de millimètre, hauteur et épaisseur 0,35 de millimètre.

Rapports et Différences. - Cette espèce est bien distincte et ne peut être confonduc avec aucune de ses congénères connues.

Gisement et Localités. - Elle est très-rare dans les couches à Bryozoaires du terrain Maestrichtien, et n'a été trouvée qu'à Bemelen.

13. GYTHere MULtilamella nov. spec. 1853.

Pl. V, fig. $12 a, b, c, d$.

Valves ovales-oblongues, sub-tétragonales; à bord antérieur sub-sémicirculaire et marginé d'une partie comprimée très-étroite; terminées en arrière par un lobe comprimé, obliquement tronqué, quadridenté et assez fortement tourné vers le côté pectoral. Leurs bords inférieur et supérieur sont droits et divergents en avant. La partie voûtée est convexe, obliquement déprimée et obscurement bilobée en arrière, Elle se rattache au bord supérieur et à la partie comprimée 
antérieure, par une pente assez donce, tandis qu'elle se raccorde à la partie comprimée postérieure et à celle du bord inférieur par une pente très-rapide. La surface du dos des valves offre un tubercule sub-central oblong, oblique et très-bien marqué; elle est couverte de plusieurs lames flexueuses, séparées par des sillons assez profonds. Ceux-ci sont ornés de nombreux points creux anguleux et inégaux. Les lames paraissent être originairement au nombre de trois: elles partent d'un point, situé presque immédiatement en avant du lobe comprimé postérieur, vers le quart postéricur de la longueur totale des valves; de ce point elles se dirigent en avant, en longeant le bord inférieur, jusqu’à ce que ayant dépassé le tubercule sub-central, elles so recourbent assez brusquement en haut et en arrière, après s'être bifurquées en plusieurs endroits. Trois de ces lames, c'est-à-dire toutes celles qui se dirigent en arrière, se bifurquent de nouveau, immédiatement au delà du tubercule sub-central, tandis que toutes les antres vont se terminer sur le tiers antérieur du bord supérieur.

La carapace offre vers le tiers postérieur de sa longueur une section transversale à contour ovale-ellipsoidal; en dessons elle présente un aspect ellipsoide-subpentagonal.

Dimensions. - Longueur $1 \frac{1}{20}$ de millimètre, hauteur et épaisseur 0,5 de millimètre.

Fapports et Diférences. - La Cythere Haimeanal) de l'Etage Parisien de Grignon, se rapproche de celle-ci. Elle s'en distingue néanmoins facilement par le dos de ses valves presque également haut dans toute sa longueur et rattaché à la partie comprimée antérieuro par une pente beaucoup plus douce, par les tubercules de la surface de cette dernière partie et par la forme du lobe comprimé postérieur.

Gisement et Localités. - Dans le Système Sénonien à Bulkomsdal et dans le calcaire grossier à silex gris à St. Pierre, Duché de Limbourg; en Belgique elle se trouve dans le Système Maestrichtien à Folx-les-Caves et dans le Système Sénonien à Ciply près Mons.

14. Cythere PUncturata nov. spec. 1853.

PI. VI, fig. $1 a, b, c, d$.

Les valves de cette Cythere sont allongées sub-tétragonales; leur bord antérieur est obliquement sémi-circulaire, et marginé d'une partie comprimée assez large, dont la surface est bisillonnée; elles sont terminées en arrière par un lobe comprimé obliquement tronqué, tourné vers le côté pectoral, et d'une largeur presque égale à celle de la partie comprimée antérieure; les bords supérieur et inférieur sont presque droits, peu divergents en avant, et le dernier est

1) Bosquet, 1850. Description des Entonostracés fossiles des terrains tertiaires de la France et de la Belyique. pp. 61, 62, pl. II, fig. $14 a-d$. 
faiblement excavé. La voûte dorsale des valves est obscurement bilobée en arrière; elle se rattache au bord supérieur et à la partie comprimée antérieure par une pente assez douce et se raccorde au bord inférieur et au lobe comprimé postérieur par une pente très-rapide. 'Toute sa surfacc est creusée de points allongés et disposés par séries flexueuses. Les tubercules subcentraux sont peu proéminents. Le long de la partie comprimée antérieure on remarque quelques points creux arrondis ou oblongs, plus grands que ceux du dos des valves. Les régions pectorale et dorsale sont creusées de points, pareils à ceux de toute la partie voûtée.

Le bouclier vu par l'extrémité antérieure offre un aspect deltoïde: vu en dessus ou en dessous, il offre un aspect sub-cunéiforme.

Dimensions. - Longueur 0,8 de millim., hauteur 0,4 de millim. et épaisseur 0,3 de millim.

Bapports et Différences. - Elle se rapproche de ma Cythere interrupta, mais s'en distingue nettement par la disposition des lignes ponctuées de sa surface, par la voûte dorsale de ses valves moins haute, par le manque des deux sillons longitudinaux et par son lobe comprimé postérieur.

Gisement et cocalités. - Elle est peu commune dans le Système Maestrichtien à St. Pierre, à Gronsveld, à Bemelen, à Geulhem et à Fauquemont. Elle est très-rare dans le Système Sénonien (calcaire grossier ì silex gris) entre St. Pierre et petit Lanaye. Dans cettc dernière couche crétacée, elle se rencontre aussi en Belgique à Frère pres 'Tongres.

15. GYTHERE VESiculosa nov, spec. 1853.

Pl. VI, fig. $2 a, b, c, d$.

Cette espèce a des valves oblongues, sensiblement pentagonales, à bord antérieur obliquement arrondi et marginé d'un limbe comprimé lisse; terminées en arrière par un lobe fortement comprimé, assez large, d'une forme tétragonale et tourné vers le côté pectoral. La partie voûtée est très-enflée; elle se rattache au bord antérieur par une pente assez douce, rejoint le lobe comprimé postérieur par une pente rapide, et retombe perpendiculairement sur le bord pectoral. Sa surface est creusée de plusieurs sillons flexueux ponctués, qui dessinent 10-14 bourrelets peu saillants. La plupart de ces bourrelets sont simples, quelques-uns bifurqués, tous s'effacent sur la région pectorale. Les tubercules sub-centraux sont bien marqués et arrondis en arrière. A côté des deux tubercules cardinaux, le bord valvaire fait une légère saillie.

La carapace offre, vers le quart postérieur de sa longueur, une section transversale à contour trigone. La région pectorale présente un contour piriforme.

Dimensions. - Longueur 0,7 de millim., hauteur 0,35 de millim. et épaisseur 0,42 de millim.

Ikapports et Différences. - Cette espèce se distingue au premier obord de la précédente, 
par son lobe comprimé postérieur plus large, par la disposition des sillons de sa surface, et surtout par le renflement de sa partie voûtée.

Gisement et Localités. - Cette Cythere est rare et n'a été rencontrée jusqu’à présent qu’à Gronsveld, à St. Pierre et à Nedercanne, dans les couches à Bryozoaires du Système Maestrichtien.

16. GYTHERE GEREBRALIS nov, epec. 1853.

Pl. VI, fig. $3 a, b, c, d$.

Les valves de cette très-petite espèce sont oblongues; leur contour et sub-tétragonal; leur bord dorsal est faiblement sinué au milieu, le pectoral parait crénelé par la saillie des bourrelets de la surface; le postérieur est obliquement arrondi, et marginé d'une partie comprimée très-étroite, munie de quatre petites dents, qui sont fortement tournées vers le côté pectoral; l'antérieur est aussi obliquement arrondi, mais marginé d'un limbe comprimé assez large, se terminant brusquement, un peu en avant de la moitié du côté pectoral, par un renflement assez saillant. De ce renflement pectoral part un sillon transversal profond, qui va se terminer en avant $d u$ tubercule sub-central. Celui-ci est lisse, peu proéminent et arrondi. La partie voûtée est le plus hante vers le tiers postérieur de sa longueur; de ce point, elle se rattache au bord supérieur et au limbe comprimé antérieur, par une pente assez donce, (qui cependant devient très-rapide à proximité de cette dernière partie); elle rejoint le lobe comprimé postérieur par une pente très-rapide et retombe perpendiculairement sur le bord inférieur. Toute la surface ie la voûte dorsale des valves est creusée de sillons flexueux, qui dessinent des bourrelets peu visibles (même sous une forte loupe) vers la partie supérieure et médiane, mais bien prononcés vers les côtés postérieur et inférieur.

La carapace présente, vers le quart postérieur de sa longueur, une section transversale à contour arrondi-subtrigone. Le côté pectoral présente un aspect ovale sub-cunéiforme, et montre l'étranglement produit par le sillon mentionné plus haut.

Dimensions. - Longueur 0,5 de millimètre, hauteur et épaisseur 0,3 de millimètre.

Itapports et Différences. - Cette Cythere offre des caractères tellement tranchés, qu'elle ne saurait être confondue avec aucune autre.

Gisement et Localités. - Elle semble être extrémement rare. Je n’en ai trouvé qu'un seul individu bivalve, dans les couches à Bryozoaires du Système Maestrichtien, à Bemelen, Duché de Limbourg. 
17. GYTHERE GIBBERULA nov. spec. 1853.

PI. VI, fig. $4 a, b, c, d$.

Valves très-allongées, à bord antérieur plus que sémi-circulaire; elles sont obliquement bitronquées en arrière, et terminées par un lobe comprimé très-petit et très-étroit; leurs bords supérieur et inférieur sont presque droits, le dernier est faiblement sinueux. Leur voûte dorsale est le plus haute vers le quart postérieur de sa longueur totale; de ce point elle se rattache au bord antérieur par une pente assez douce, elle se raccorde au bord supérieur par une pente rapide, rejoint le lobe comprimé postérieur par une pente très-rapide, et retombe presque perpendiculairement sur le bord inférieur. Un peu en arrière du point le plus elevé de la partie voûtée, on remarque un étranglement qui, après avoir parcouru obliquement la surface de cette partie, va se terminer à côté de l'oreillette cardinale antérieure. Entre cet étranglement et le bord supérieur, on distingue quelques sillons transversaux obsolètes.

Le bouclier présente une section transversale à contour deltoïdal et une section longitudinale à contoưr tétragono-cunéiforme.

Dimensions. - Longueur 0,65 de millimètre, hauteur 0,25 de millimètre et épaisseur 0,3 de millimètre,

Gisement et Localités. - A Bemelen, dans le terrain Maestrichtien. Elle est très-rare.

18. Gythere STraingulata nov. spec. 1853.

PI. VI, fig. $5 a, b, c, d$.

Cette singulière Cythere a des valves oblongues-subpentagonales, obliquement arrondies aux extrémités, terminées en arrière par un lobe comprimé étroit et obtus; à bords inférieur et supérieur presque droits et parallèles. Leur voûte dorsale et très-haute et se rattache aux bords antérieur et supérieur, ainsi qu’au lobe comprimé postérieur, par une pente très-rapide; elle retombe perpendiculairement sur le bord inférieur. Vers le milieu du bord supérieur on remarque une petite échancrure, d'où part un sillon transversal, qui va se terminer sur la région pectorale. Ce sillon sépare le dos des valves, qui est assez fortement étranglé en cet endroit, en deux parties distinctes. La partie postérieure est divisée à son tour en deux parties par un sillon longitudinal, qui se termine en arrière du sillon transversal. Entre le sillon longitudinal et le bord supérieur, on aperçoit encore quelques sillons transversaux obsolètes.

Le bouclier offre une section transversale à contour deltoïdal. Quand on l'examine du côté supérieur ou inférieur on observe le mieux le fort étranglement médian, qui m’a engagé à lui donner le nom de strangulata. 
Dimensions. - Longueur 0,5 de millimètre, hauteur et épaisseur 0,3 de millimètre.

Rapports et Différences. - Cette espèce est très-remarquable et facile à distinguer des autres Cythere.

Gisement et Locallités. - Elle est fort rare; je n'en ai trouvé qu'un seul échantillon bivalve, dans les assises supérieures du Système Maestrichtien à Fauquemont.

19. CYTHERE UMBONELLA nov. spec. 1853.

Pl. VI, fig. $6 a, b, c, d$.

Les valves de cette espèce sont allongées, obscurement pentagonales; leur bord antérieur est obliquement sub-sémicirculaire et marginé d'un limbe comprimé assez large, dont la surface est creusée de points anguleux; elles sont terminées en arrière par une partie comprimée trèslarge, obliquement sub-trigone et dont la surface offre un grand nombre de points creux, irrégulièrement anguleux et très-rapprochés; leur voûte dorsale est sub-carénée le long du côté pectoral, et brusquement terminée en arrière par un renflement obtus. De ce point elle est rattachée au bord-antérieur par une pente douce, et retombe perpendiculairement sur le lobe comprimé postérieur. Vers le tiers postérieur de la longueur totale du bord supérieur, on remarque une petite échancrure, d'oú part un sillon large et profond, qui sépare en cet endroit la partie voûtée de la partie comprimée postérieure, en donnant naissance à une étranglement fort remarquable. La surface de la parte voûtée est creusée de points peu profonds.

La carapace présente une section transversale à contour deltoide, dont deux côtés sont concaves; la région pectorale affecte une forme sagittato-hastée.

Dimensions. - Longueur 0,7 de millimètre, hauteur 0,4 de millimètre et épaisseur 0,3 de millimètre.

Fapports et mifrérences. - La Cythere umbonata Wiluiamson ${ }^{1}$ ) des couches crétacées de l'Angleterre, présente vers le tiers postérieur de la longueur de chaque valve, une protubérance ou plutôt une épine conique, beaucoup plus haute et plus pointue; cette épine est en oûtre placée sur la ligne longitudinale médiane des valves, et la surface de celles-ci offre des points creux non anguleux et disposés concentriquement.

Gisement et Localités. - Dưns le Système Maestrichtien à Bemelen, et dans le Système Sénonien (calcaire grossier à silex gris) à St. Pierre, très-rare. Je n'en possède plus que deux valves séparées. Mon meillieur échantillon, celui d'après lequel est fait le dessin, est cassé.

1) WrLlisuson, 1847. Transactions Manchester Literary and Philosoplical Society Vol. VIII, pag. 79, pl. IV, fig. 78 . 
20. GYTHERE LONGISPINA nov. epec. 1853.

Pl. VI, fig. $7 a, b, c, d$.

Valves allongées-sub-ovales; à bord antérieur sub-semicirculaire, et marginé d'une partie comprimée unisillonnée; offrant en arrière une partie comprimée trigone, se terminant en une pointe aiguë. Cette pointe est située en dessus de la ligne longitudinale médiane. Le bord supérieur est droit. La partie voûtée est tellement enflée le long du côté inférieur, qu'elle dépasse de beaucoup le bord, et que ce côté semble fortement arqué; elle présente un étranglement transversal oblique, et porte vers le tiers postérieur de sa longueur, à côté du bord inférieur, une épine très-longue, presque droite, ct faiblement inclinée vers le côté inférieur.

La carapace, vue par l'extrimité antérieure, offre un aspect triangulaire. Vue en dessous, elle présente un aspect hasté-sub-tétragonal. La région pectorale est creusée en avant de six sillons longitudinaux, dont trois sur chaque valve.

Dimensions. - Longueur 0,8 de millim., hauteur 0,4 de millim. et épaisseur 0,6 de millim.

Itapports et Difrérences. - C'est une espèce des plus distinctes. La longue épine du dos de ses valves la rend fort remarquable.

Gisement et Localités. - La Cythere longispina est très-rare dans la craie blanche sans silex (Système Sénonien Duront) à Slenaken et à Pesaken (Duché de Limbourg). Elle se trouve en Belgique dans la même couche crétacée à Heure-le-Romain.

21. CYTHERe Machophthalma BosQdet, 1847 (an JoNes?).

Pl. IX, fig. $4 a, b, c, d$.

Cythere macrophthalma Bosquet, 1847. Descript. des Entomostr. foss. de la craie de Maestriclet. pag. 16, 17, pl. III, Hig. $3 a-d$.

" Bosouet, 1847. Mémoires de la Société Royale des Sciences de Liége. tom. IV, pp. 366, 367, pl. III, fig. 3.

Cythereis " ? Jones, 1849. A Monograph of the Entomostr. of the cretaceous form. of England. pag. 17, tab. II, fig $8 a-b^{\prime \prime \prime}$.

Valves déprimées, allongées-subpentagonales, obliquement arrondies en avant, et terminées en arrière par un lobe comprimé subtrigone; leur côté pectoral est faiblement sinué au milieu; leur oreillette cardinale antérieure est très-developpée et produit une saillie très-forte à l'extrémité antérieure du bord supérieur. Leur voûte dorsale est sub-carénée le long du côté inférieur, et la carène se termine en arrière par une petite épine; c'est immédiatement en avant de cette 
dernière, que la partie voûtée est le plus haute; de ce point elle rejoint la partie déprimée antérieure et le bord supérieur par une pente assez douce; elle se rattache ì la partie comprimée postérieure par une pente très-rapide et retombe presque perpendiculairement sur le bord inférieur. Sa surface est lisse. Le tubercule sub-central, qui est ovale-oblong, y fait une saillie assez forte; immédiatement en arrière, on remarque un bourrelet oblong, moins gros que ce tubercule, et ayant, à peu près comme lui, une direction oblique.

Le bouclier offre vers le tiers postérieur de sa longueur une section transversale à contour subtrigone. Vu par le côté pectoral il présente un aspect ovale-lancéolé.

Quelques valves ont conservé leur aspect corné; elles sont demi-transparentes et présentent près du bord inférieur une grande tache rose. Une tache de même couleur, mais beaucoup plus petite, se remarque près du bord antérieur.

Je doute beaucoup que la Cythere (Cxthereis) macrophthalma de Mr. Jones soit bien la même que celle-ci.

Dimensions. - Longueur 0,85 de millimètre, hauteur et épaisseur 0,55 de millimètre.

Rapports et Différences. - Elle se rapproche un peu de la Cythere (Crthereis) interrupta Jones, qui devra recevoir un nouveau nom, parcequ'elle diffère de celle que j’avais décrite antérieurement sous ce même nom spécifique.

Gisement et Localités. - Cette Cythere se rencontre assez rarement dans le Système Naestrichtien à St. Pierre et à Kunraede; et dans le Système Sénonien (calcaire grossier à silex gris) entre St. Pierre et Petit-Lanaye, dans le Duché de Limbourg. Elle est moins rare dans la craie blanche avec silex à Heure-le-Romain, et se rencontre aussi dans le Système Maestrichtien à Wonck, à Sichen et à Jandrain en Belgique. Je viens de la trouver aussi dans une roche crétacée, ayant une ressemblance parfaite avec le calcaire Maestrichtien et provenant d'ffö, (Schoonen) en Suède.

22. GYThere SAgitTATA nov. spec. 1853.

Pl. VT, fig. $\delta a, b, c, d$.

Cette Cythere a des valves très-allongées, à bord antérieur obliquement sémi-circulaire et marginé d'un bourrelet aplati assez large; terminées en arrière par un lobe comprimé fort grand, d'une forme sub-trigone, arrondi en arrière et tourné vers le côte pectoral; à bords supérieur et inférieur presque droits et faiblement divergents en avant. Leur voûte dorsale, qui se rattache aux bords antérieur et supérieur par une pente assez douce, rejoint le lobe comprimé postérieur par une pente très-rapide et retombe presque perpendiculairement sur le bord inférieur; sa surface est divisée en trois bourrelets longitudinaux inégaux par deux sillons larges; les bords 
de ces sillons sont ornés de points creux assez rapprochés les uns des autres; sur le bourrelet comprimé antérieur on remarque une série de points creux pareils; sur le lobe comprimé postérieur on observe encore quelques points plus grands et anguleux; sur chacun des deux bourrelets longitudinaux supérieurs on aperçoit une ligne creuse; le bourrelet inférieur, qui se confond avec l'antérieur, est creusé de trois à quatre lignes semblables. Le bourrelet médian est épaissi et lisse an milieu. La région dorsale est assez large et creusée de six points. La carapace vue par l'extrémité antérieure, offre un aspect deltoïde. La région pectorale est aplatie, sagittiforme et sa surface est creusée de plusieurs lignes.

Dimensions. - Longueur 0,8 de millim., hauteur 0,35 de millin. et épaisseur 0,4 de millim.

Razports et diférences. - Aucune des autres Cythere connues, dont le dos des valves est tricosté, n'a comme celle-ci la région pectorale sagittiforme. On̂tre cette différence importante, elle présente un second caractère qui l'eloigne des autres espèces à valves tricostées: ce caractère consiste dans la grandeur du lobe comprimé postérieur.

Gisement et Localités. - Elle est extrèmement rare; je n'en ai trouvé que deux échantillons; l'un dans le Système Maestrichtien de Bemelen (Duché de Limbourg), et l'autre dans les assises supérieures du Système Sénonien (calcaire grossier à silex gris) de Ciply près Mons (en Belgique).

23. CYTHERE ORCHIDEA nov, spec, 1853.

Pl. VI, fig. $9 u, b, c, d$.

Cette espèce a des valves allongées, à bord antérieur obliquement sémicirculaire et marginé d'un limbe en forme de bourrelet; terminées en arrière par un lobe comprimé subtrigone, trésgrand, finissant postérieurement en pointe. Cette partie est très-remarquable par une petite gibbosité, qui forme une saillie vers le côté pectoral, et qu'on n'aperçoit parfaitement que qunnd on examine la carapace en dessous (c'est l'ensemble de ces deux gibbosités qui m'a engagé à lui donner le nom qu'elle porte). Les bords supérieur et inférieur des valves sont presque droits et divergents en avant. La partie voûtée est lisse, déprimée et sub-carénée le long du côté pectoral; elle offre vers le milieu un pli longitudinal aigu, droit et peu saillant dans sa moitié inférieure, plus saillant, plus épais et oblique en avant, c'est-à-dire où il porte lo tubercule sub-central.

La carapace vue par l'extrémité antérieure présente un aspect trigone-sub-pyramidal. La région dorsale est aplatie, assez large et d’une forme lancéolée; la région pectorale est aplatie cunéiforme et ornée à sa surface de deux bourrelets, tronqués en arrière, et convergents en arant, d'oú nait un relief en forme de $V$ renversé. 
Dimensions. - Longueur 0,75 de millim., hauteur 0,4 de millim. et épaisseur 0,3 de millim.

IRapports et Difrérences. - La conformation du lobe comprimé postérieur de cette espèce, le rélief de la surface de la région pectorale, et surtout les deux protubérances à l'extrémité postérieure du bord pectoral, suffisent pour la distinguer au premier abord.

Gisement et Localités. - Cette singulière Cythere n’a été trouvée jusqu'ici que dans les couches à Bryozoaires du Système Maestrichticn à Nedercanne, à St. Pierre, à Bemelen, à Geulhem et à Fauquemont.

24. CYTHERE COMPLANATA nov. spec, 1853.

Pl. VI, fig. $10 a, b, c, d$.

Valves allongées, fortement déprimées, ayant le bord antérieur marginé, obliquement subsémicirculaire et muni dans sa moitié inférieure de 8-9 dents tronquées, très-courtes et trèsrapprochées; elles sont terminées en arrière par un lobe comprimé sub-quadrangulaire, tourné vers le côté pectoral et garni de quatre petites dents, semblables à celles du bord antérieur; les bords supérieur et inférieur sont presque droits, très-faiblement sinueux, et très-peu divergents en avant; le premier est terminé en arrière par une pointe saillante et aiguë; tandis que le dernier est marginé d'un bourrelet, qui est brusquement terminé en arrière, vers le quart postérieur de la longueur totale des valves, et qui se confond antérieurement avec le rehord marginal. Toute la surface est lisse, et le tubercule sub-central peu saillant est arrondi en arrière.

La carapace presente une section transversale à contour tétragone-allongé. La région dorsale est étroite, en forme de coin et tronquée en arrière.

Dimensions. - Longueur 0,6 de millim., hauteur 0,25 de millim. et épaisseur 0,15 de millim.

Gisement et Localités. - Cette Cythere est très-rare dans les couches à Bryozoaires du Système Maestrichtien. Je ne l'ai trouvée que dans la seule localité de Bemelen.

25. CYTHERE LEPIDA nov. spec. 1853.

PI. VI, fig. $11 a, b, c, d$.

Valves déprimées, assez épaisses, obovales-sub-hexagonales; fortement élargies dans le quart antérieur de leur longueur; elles sont arrondies en avant et terminées en arrière par un lobe comprimé sub-triangulaire, muni de quatre dents très-courtes, obtuses et tournées vers le côté pectoral; l'oreillette cardinale antérieure est très-grande et offre une très-petite échancrure marginale. Le tubercule sub-central des valves est assez grand, ovalaire et lisse; le rebord marginal antérieur se confond avec un bourrelet épais, arrondi et lisse, qui borde le côté inférieur et se termine brusquement vers le quart postérieur de la longueur totale des valves; un autre bour- 
relet, noduleux dans sa moitié postérieure, borde le côté supérieur, et se termine à peu près ¿ la même hauteur. Ln avant de la grosse protuberance sub-centrale, on remarque cinq petits tubereules arrondis et assez écartés les uns des autres; une série de 8-9 tubercules pareils se remarque le long du bord antérieur; enfin, deux autres séries obliques, formées de tubercules semblables, se montrent en arrière des tubercules subcentraux et se prolongent jusque sur le bourrelet marginal supérieur.

La carapace présente une section transversale à contour tétragone-allongé, à bords latéraux simués. La région dorsale est aplatie; elle offre un contour cunéiforme et est ornée de quelques sillons transversaux obliques, dont les quatre antérieurs sont opposés en croix.

Dimensions. - Longueur 0,85 de millim., hauteur 0,5 de millim. et épaisseur 0,35 de millim.

Gisement et Localités. - Cette élégante Cythere est rare. Elle se rencontre dans les couches à Bryozoaires du terrain Maestrichtien à St. Pierre, à Geulhem et à Bemelen. Je viens de la trouver aussi dans le calcaire Maestrichtien de Kunraede.

26. CYTHERE QUADRIDENTATA: nov. spec, 1853.

Pl. VI, fig. $12 a, b, c, d$.

Les valves de cette Cythere sont allongées, subpentagonales; leurs bords supérieur et inférieur sont presque droits, faiblement divergents en avant; elles sont obliquement arrondies et marginées en avant d'un rebord comprimé assez large et muni dans sa moitié inférieure de 6 dents, dont deux courtes, et quatre plus longues, obtuses et dirigées vers le côté pectoral; elles sont terminées en arrière par un lobe comprimé subtétragone et garni sur son bord de quatre dents obtuses, égales aux quatre grandes dents du bord antérieur, et dirigées comme elles vers le côté pectoral; la voûte dorsale des valves est déprimée et retombe presque perpendiculairement sur le bord inférieur. Le tubercule subcentral est ovale-oblique et orné de deux sillons longitudinaux. La moitié postérieure de la partie voûtée est divisée longitudinaleinent en deux lobes d'une forme tétragone-allongée; l'un de ces lobes présente supérieurement deux sillons obliques, tandis que l'autre est partagé en plusieurs bourrelets de forme et de grandeur très-inégale, par trois à quatre sillons flexuenx. Le tubercule cardinal antérieur est placé sur une oreillette nettement détachée du reste de la surface et faiblement échancrée sur son bord. La région dorsale est aplatie, et offre dans sa moitié postérieure, qui est élargie, six sillons transversaux, dont trois à chaque côté de la ligne de jonction des valves. La région pectorale est allongée, subcordiforme et sa surface est omée de six sillons, dont deux longitudinaux et quatre transversaux obliques.

Dimensions. - I Iongueur 0,6 de millim., hauteur 0,35 de millim. et épaisseur 0,25 de millim. 
Gisement et Localités. - Les couches à Bryozoaires du Syst. Maestrichtien à Bemelen, à St. Pierre et à Nedercanne. En Belgique, dans les assises inférieures du même Système à Folx-les-Caves.

27. CYTHERE ARENOSA nov. spec. 1853.

Pl. VII, fig. $1 a, b, c, d$.

Cette espèce a des valves ovales-elliptiques; à bord antérieur obliquement sub-sémicirculaire et marginé d'un rebord comprimé lisse, assez large, se confondant supérieurement avec l'oreillette cardinale antérieure. Cette dernière fait en cet endroit une saillie bien marquée. Le dos des valves est fortement enflé, et toute sa surface est couverte de grains, ou plus souvent de squames, couchées vers les bords, devenant plus grandes sur la moitié postérieure et disposées par séries concentriques. Les tubercules subcentraux sont arrondis et très-peu proéminents. Quand on examine la carapace en dessus, on remarque qu'à côté de l'oreillette cardinale antérieure, elle offre une partie fort large et quadrongulaire, et que la région dorsale est ornée, i chaque côté de la ligne de jonction des valves, de quatre sillons marginaux et obliques.

La carapace présente une section transversale à contour sub-cordiforme, et une section longitudinale à contour obovale.

Bimensions. - Longueur 0,55 de millim., hauteur 0,35 de millim. et épaisseur 0,36 de millim.

reapports et Difrérences. - Elle se distingue de la Cythere scabra ron Munster, ${ }^{1}$ ) des étages Subapennin et Falunien (D'Orbignr), par ses valves beancoup plus convexes, par ses extrémités non dentelées et par sa surface couverte de petites squames et non de tubercules.

Gisement et Localités. - Dans le Système Maestrichtien à Nedercanne, à St. Pierre, à Gronsveld, à Bemelen, à Geulhem et à Vieux-Fauquemont, et dans le Système Sénonien (calcaire grossier à silex gris) entre St. Pierre et Petit-Lanaye.

28. CYTHERE VARTOLATA nov. spec. 1853.

Pl. VII, fig. $2 a, b, c, d$.

Les valves de cette belle Cythere sont oblongues, elliptico-tétragonales; leur bord supérieur est droit, l'inférieur faiblement sinué; leurs deux extrémités sont largement arrondies. Elles sont marginées en avant d'une partie comprimée, dont le bord est garni d'un grand nombre de très-petites dentelures et dont toute la surface est ornée de plusieurs tubercules assez gros et

1) Von Münster, 1830. Jahrbucher für Míneralogie, Geologie, etc. von Leoniard und Bronv. pag. 63.

Bosever, 1850. Description des Entomostracés fossiles des terrains tertiaires de la France et de la Belgique pp. 105,106 , pl. V, fig. $7 a-d$. 
arrondis; elles sont terminées en arrière par une partie comprimée assez large, dont la surface est tuberculeuse, et dont le bord est muni de 5 dents aiguës, distantes et plus grandes que celles du bord antérieur. La partie voûtée est fortement convexe; elle se rattache aux parties comprinées des extrémités et au bord supérieur par une pente rapide et retombe presque perpendiculairement sur le bord inférieur. Toute sa surface est couverte d'un très-grand nombre de petits tubercules, entre lesquels on en remarque quelques-uns qui sont plus gros. Les deux oreillettes cardinales, quoique peu saillantes, sont cependant fort bien prononcées. Le long du côté inférieur on observe une série moniliforme, composée de petits tubercules très-rapprochés. Les tubercules subcentraux sont peu proéminents et anguleux en arrière.

La région dorsale assez large est ornée de deux séries longitudinales de gros tubercules arrondis et très-rapprochés; la région pectorale offre environ 8 séries longitudinales moniliformes, composées de tubercules plus petits et très-rapprochés. Une seule de ces séries moniliformes s'aperçoit le long du côté pectoral, quand on examine les valves en dessus.

Le bouclier présente une section transversale à contour ovale-arrondi.

Dimensions. - Longueur 0,65 de millimètre, hauteur et épaisseur 0,35 de millimètre.

Tapports et Difrérences. - Quoique les valves de cette espèce ayent quelque ressemblance avec celles de ma Cythere formosa ${ }^{1}$ ) de l'Etage Parisien, elle s'en distingue cependant nettement par les tubercules de la surface inégaux et beaucoup plus nombrenx, et surtout par les ornements des régions dorsale et pectorale.

Gisement et Localités. - Le Système Maestrichtien à Nedercanne, à St. Pierre et à Bemelen.

29. GYTHERE HIEROGLYPHIGA BOSQUET, 1853.

Pl. IX, fig. $5, a, b, c, d$.

Cypridina hieroglyphica Bosquer, 1847. Description des Entomostracés fossiles de la craie de Maestricht. pp. 17, 18, pl. III, fig. $4 a-d$.

Boseuet, 1847. Mémoires de la Sociéte royale des Sciences de Liége. tom. IV, pag. 367, 368, pl. III, fig. $4 a-d$.

Cythereis "Jones, 1849. A Monograph of the Entomostraca of the Cretaceous formation of England. Appendix pag. 36.

Les valves de cette Cythere sont déprimées, leur contour est allongé, obscurement pen-

1) BosQuet, 1850. Description des Entomostracés fossiles des terrains tertiaires de la France et de la Belgique. pp. 108, 109, pl. V, fig. $11 a-d$. 
tagonal; leurs bords supérieur et inférieur sont presque droits et à peu pres parallèles; l'antérieur est obliquement arrondi et marginé d'un bourrelet aplati, qui offre, au côté interne, une série de tubercules, et à son bord externe 12-14 dentelures très-petites, aignës et courtes. Les valves sont terminées en arrière par un lobe comprimé subtrigone, marginé et garni sur la moitié inférieure de son bord de 5-6 dentelures pointues, en égales en grandeur à celles du bord antérieur. La partie voûtée est presque également haute dans ses deux tiers postérieurs; elle rejoint les bords antérieur et supérieur par une pente peu rapide et retombe presque perpendiculairement sur le bord inférieur et sur le lobe comprimé postérieur. Les tubercules subcentraux sont orbiculaires, gros et lisses. Le bord pectoral de chaque valve est marginé d'un bourrelet droit, élargi au milieu, et creusé de lignes ponctuées et flexueuses; quatre bourrelcts analogues, dont deux sont réunis à la base, ornent la partie restante de la moitié postérieure du dos des valves. Les trois bourrelets supérieurs forment par leur réunion un lobe bien prononcé, qui prend naissance à côté et en arrière du tubercule subcentral. Les sillons qui séparent ces bourrelets sont parfois ponctués, et les bourrelets eux mêmes sont en général couverts de nodosités obliques. Entre le tubercule subcentral et le rebord comprimé antérieur on remarque quelques proéminences oblongues.

La carapace présente vers le tiers postérieur de sa longueur une section transversale à contour subtrigone. La région dorsale est ornée de quelques sillons obliques, à chaque côté de la ligne de jonction des bords valvaires. La région pectorale est en forme de poire ou en forme de coeur allongé; toute sa surface est creusée de lignes longitudinales onduleuses.

Dimensions. - Longueur 0,9 de millimètre, hauteur et épaisseur 0,5 de millimètre.

Rapports et Bifrérenes. - La Cythere hieroglyphica est une des espèces les plus remarquables et les plus distinctes. Elle est peu sujette à varier et s'éloigne de toutes les Cythere connues par la conformation des bourrelets de la surface.

Gisement et Localités. - Cette élégante espèce n'est pas rare et se trouve dans les couchès à Bryozoaires du Système Maestrichtien à St. Pierre, à Gronsveld, à Keer, à Bemelen, ¿ Geulhem, à Fauquemont, et à Kunraede et dans le calcaire grossier à silex gris (Système Sénonien) entre St. Pierre et Petit-Lanaye.

30. CYTHERE LABYRINTHICA nov. spec. 1853.

Pl. VII, fig. $3 a, b, c, d$.

Cette espèce, qui est une des plus grandes parmi les Cythere du terrain cretacé, a des valves très-épaisses, ellipsoïdales; leur bord antérieur est obliquement sémicirculaire et marginé d'un limbe comprimé, dont la surface est creusée d'un seul sillon et dont le bord externe est 
garni de $9-10$ dents très-petites. Les valves sont terminées en arrière par une partie comprimée très-étroite et munie, dans sa moitié inférieure, de quatre dents; leur bord inférieur est droit, légèrement sinueux, et le supérieur crénelé. Leur voûte dorsale est fortement bombée; la surface de celle-ci est ornée de sillons et de points creux profonds, de forme et de grandeur si variées, qu'il serait extrêmement difficile d'en donner une idéc exacte par une description; je me bornerai donc ì dire, que les points creux manquent tout-à-fait à l'endroit occupé dans les autres espèces par le tubercule sub-central, et qu' immédiatement en arrière on observe deux séries longitudinales de ces points, dessinant les bords d'une suite d'espaces quadrangulaires, dont les postérieurs sont les plus larges, et qui sont tous étagés de telle manière, qu’ils paraissent être imbriqués; quelques espaces quarrés pareils s'observent encore le long du bord supérieur.

La région dorsale est fort large en avant, sagittée en arrière, ot sa surface est subimbriquée dans la moitié postérieure; le tubercule cardinal postérieur est très-gros. Le côté pectoral est creusé de plusieurs sillons flexueux. La carapace présente, vers le tiers postérieur de sa longueur, une section transversale à contour arrondi-subcordiforme; elle offre une section longitudinale à contour ovalaire.

mensîns. - Longueur 1,2 de millimètre, hauteur et épaisseur 0,6 de millimétre.

Rapports et miférences. - La Cythere labyrinthica est une des espèces les plus distinctes, et offre des caractères tellement tranchés, qu'elle ne peut être confondue avec aucune de ses nombreuses congénères. Dans cette espèce et dans la précédente, je n’ai pas encore trouvé d'individus luisants, d'un aspect corné et demi-transparents, comme on en rencontre fréquemment chez les autres Cythere. Les Cythere labyrinthica et hieroglyphica sont constamment opaques et d'un blanc mat, ou d'un blancojaunâtre lorsqu'elles sont colorées par l'oxide de fer.

Gisement et 耳ocalités. - Cette interressante espèce n'est pas rare dans le Système Maestrichtien à St. Pierre, à Nedercame, à Gronsveld, à Keer, à Geulhem et à Fauquemont; elle est très-rare dans ce même Système à Kunraede.

31. Cythere Elegantula nov. spec. 1853.

Pl. V'II, fig. $1 \cdot a, b, c, d$.

Valves allongées-ellipsoïdales; à bord antérieur plus que sémi-circulaire, et marginé d'un limbe comprimé dont toute la surface est couverte de gros tubercules arrondis; terminées en arrière par une partie comprimée obliquement tronquée et à surface lisse. Leurs bords pectoral et dorsal sont presque droits, le premier est marginé d'un limbe comprimé très-étroit, tandis que le dernier est faiblement sinué en arrière. Leur vôtte dorsale est presque également haute 
dans ses deux tiers postérieurs; elle est rattachée par un pente assez douce au bord supérieur et à la partie comprimée antérieure; elle rejoint la partie comprimée postérieure par une pente rapide et retombe presque perpendiculairement sur la partie marginale du côté inférieur. Le long de cette dernière partie on remarque une série moniliforme, composée de petits tubercules très-rapprochés; plusieurs autres séries de tubercules, également moniliformes, et deux côtes longitudinales inégales et lisses décorent le reste de la surface. De ces deux côtes longitudinales, l'une, plıs courte, borde le côté supérieur, tandis que l'autre, qui est beaucoup plus large dans ses deux tiers antérieurs que dans son tiers postérieur, prend naissance immédiatement en arriére de la partie comprimée antérieure, et va se terminer en avant du lobe comprimé postérieur.

La carapace présente vers le tiers postérieur de sa longueur une section transversale ì contour subtrigone.

Dimensions. - Longueur 0,5 de millim., hauteur 0,4 de millim. et épaisseur 0,32 de millim.

IRapports et Diférences. - Quoique cette belle espèce ait quelques rapports avec ma Cythere moniliferal) de l'Etage Falunien des environs de Bordeaux, elle s'en distingue cependent au premier abord par la disposition des séries tuberculeuses de sa surface, par sa partie comprimée antérieure garnie d'une série de gros tubercules, et surtout par ses deux côtes longitudinales lisses.

Gisement et Localités. - Elle parait être extrèmement rare; je n'en ai trouvé jusqu'à présent qu'un seul échantillon bivalve dans le terrain Maestrichtien de Kunraede.

32. CYTHERE HORRIDULA nov. spec, 1853.

P1. VII, fig. $5\|, b, c$,$\| .$

Valves allongées, à bord antérieur plus que sémicirculaire et marginé d’une partie comprimée, dont la surface est ornée de gros tubercules arrondis et dont tout le bord est garni d'un très-grand nombre de petites dents aiguës, disposées sur deux rangs; elles sont terminées en arrière par un lobe comprimé subtrigone, qui porte trois dents, plus grandes que celles du bord antérieur et dirigées vers le côté pectoral; les côtés inférieur et supérieur sont ároits et divergents en avant; l'oreillette cardinale antérieure est trigone et sa surface est anguleuse. Ia partie voûtée est déprimée; elle retombe presque perpendiculairement sur le bord inférieur. Une carène, surmontée d'une série de 7-8 tubercules aplatis en dessus et réunis entr'eux par des étranglements très-étroits en forme de chapelet, sépare la voûte dorsale des valves de la région

') Boseuer, 1850. Description des Entomostr. fossiles des terrains tertiaires de la France et de la Belrique. pp. 106,107, pl. V, fig. $9 a-\pi$. 
pectorale. Sur toute la surface s'élèvent plusieurs épines assez longues, couchées vers les bords, et quelques tubercules arrondis et épars, entre lesquels on remarque encore de nombreux points creux allongés. Parmi les épines qui hérissent la surface de chaque valve il y en a une, qui est beaucoup plus grande que toutes les autres, et qui est placée près de l'extrémité postérieure du bord supérieur. A côté de cette grande épine, la surface est onduleuse. Immédiatement en arrière du rebord marginal antérieur, on remarque un espace concave, offrant quelques petites côtes rayonnantes vers chacun des tubercules qui s'élèvent sur le limbe marginal. Les tubercules subcentraux sont fort saillants et pointus.

La carapace, vue par l'extrémité antérieure, offre un aspect trigone et la région pectorale un aspect piriforme.

Dimensions. - Longueur 0,8 de millimètre, hauteur et épaisseur 0,4 de millimètre.

Rapports et Différences. - La Cythere aculeata ${ }^{3}$ ) des Litages Parisien et Suessonien de la France est fort rapprochée de la Cythere horridula. Elle s'en distingue néammoins, par les épines aciculaires de la surface de ses valves; par les autres épines très-grosses et recourbées en forme d'aiguillons; par son oreillette cardinale antérieure non triangulaire et pointue, mais arrondie; par l'absence de la grande épine à côté de son oreillette cardinale postérieure, et enfin par ses régions dorsale et pectorale tres-différentes.

Gisement et Localités. - Cette belle espèce est rare, et n'a été trouvée que dans le Système Maestrichtien à St. Pierre et à Bemelen. En Belgique elle existe dans le même Système crétacé, i Ciply près Mons.

33. CYTHERE EXIMia nov. spec. 1853.

Pl. VII, fig. $6 a, b, c, d$.

Les valves de cette belle Cythere présentent un contour allongé-tétragonal. Leur bord antérieur est obliquement arrondi et marginé d'un limbe comprimé, dont la surface est lisse dans sa moitié supérieure et couverte, dans sa moitié inférieure, de 4-5 gros tubercules. Le bord du limbe est garni dans cette dernière moitié de $6-7$ dents courtes et obtuses. Les valves sont terminées en arrière par un lobe comprimé assez large, de forme subtrigone, portant sur sa moitié inférieure 4 dents, plus grandes que celles du bord antérieur. La partie voûtée est déprimée, elle se rattache au lobe comprimé postérieur par une pente très-rapide et retombe presque perpendiculairement sur le bord pectoral. Les tubercules subcentraux sont grands, sail-

1) Bosquet, 1850. Description des Entomostr. fossiles des terrains tertiaires de la France et de la Belgique. pag. 119 , pl. V, fig. $10 a-d$. 
lants et pointus en arrière. La surface de la partie voûtée est ornée en avant des tubercules subcentraux, d'un grand nombre de granulations, et est divisée en arrière de ces tubercules en trois lobes, dont l'inférieur est le plus gros et le plus saillant. Immédiatement en dessus de ce dernier, dont la surface est creusée de 3-4 sillons inégaux, on remarque trois grosses protubérances, placées sur la ligne longitudinale médiane des valves et formant le lobe médian, qui est le plus étroit. Le lobe supérieur offre ì sa surface deux protubérances pareilles aux précédentes, et cinq autres de forme différente, qui paraissent imbriquées et dont les quatre supérieures forment l'arête marginale du bord valvaire supérieur. L'oreillette cardinale antérieure est trèssaillante.

La région pectorale, qui offie un contour trigone, parait formée de plusieurs parties squamiformes imbriquées, ou du moins se recouvrant partiellement les unes les autres.

La carapace vue par l'extrémité antérieure offre un aspect tétragonal.

Dimensions. - Longueur 0,6 de millimètre, hauteur 0,35 de millimètre et épaisseur 0,3 de millimètre.

Itapports et Difrérences. - La Cythere eximia offre des caractères tellement saillants, qu'elle ne peut être confondue avec aucune de ses congénères.

Gisement et Localités. - Cette belle espèce n'est pas rare et se trouve dans le Système Maestrichtien à St. Pierre, à Nedercanne, à Bemelen, à Gronsveld, à Fauquemont, à VieuxFauquemont, à Kunraede et à Schin-op-Geul; et dans le Système Sénonien (calcaire grossier ì silex gris) à St. Pierre. Elle se rencontre en oûtre en Belgique, dans le Système Sénonien (calcaire grossier à silex gris) à Ciply près Mons, et dans la craie blanche sans silex, appartenant au même Système de Mr. Dumont, à Heure-le-Romain.

34. cythere ornatissiva Reuss, spec. 1846.

PI. IX, fig. $6 a, b, c, d$, et Var. A. Pl. VII, fig. $7 a, b, c, d$.

Cytherina quadrilatera? Ad. RoEmer, 1840. Versteinerungen des Nord-dentschen Kreidegebirges. pag. 105, tab. XVI, fig. 19.

ornatissima Reuss, 1846. Versteinerungen der Bölmischen Kreide-formation. pag. 104, tab. XXIV, fig. 18 .

ciliata Reuss, 1846. Ibidem. pag. 104, tab. XXIV, fig. 17.

echinulata WiLlianson, 1847. Transactions of the Manchester Literary and Plilosoplical Society. Vol. VIII. (Memoir on some of the IIicroscopical Objects found in the Mrud of the Levant and other Deposits. pl. IV, fig. 75,76$)$. 
Cythere harpa? Connuel, 1546. Mémoires de la Société Géologique de France. $2^{\text {me }}$ Série, tom. I, $2^{\text {me }}$ partie, pag. 199, pl. VIII, fig. 13.

Cythereis quadrilatera? Jones, 1849. A Monograph of the Entomostraca of the Cretaceous formation of England (in the Memoirs of the Palaeontographical Socicty). pag. 18, tab. III and IV, fig. $10 a-j^{\prime}$.

" ciliata Jones, 1849. Ibidem. pp. 19,20 , tab. IV, fig. $11 a-h^{\prime}$. Cypridina muricata Reuss, 185I. Entomostraceen und Foraminiferen des Kreidemergels von Lemberg (aus den Naturwissenschäftlichen Abhandlungen von WiLri. Hatdinger). Vierter Band, $1^{\text {ste }}$ Abth. pag. 50 , tab. VI, fig $12 a, b, c$.

TAR. A. NOdulosa Bosøuet. Valvis extremitatibus paucidentatis; carinâ, limboque anteriore et margine superiore, non spinis sed tuberculis magnis munitis. Pl. VII, fig. $7 a, b, c, d$.

Cette intéressante Cythere a des valves allongées, obscurement tétragonales; à bord antérieur obliquement sémicirculaire et marginé d'un limbe assez large, qui est orné à sa surface de plusieurs tubercules et qui est garni à son bord d'un grand nombre de dents marginales (les dents qui hérissent le tiers inférieur sont ordinairement plus longues que les autres). Les valves sont terminées en arrière par un lobe comprimé subtrigone, qui est marginé d'un rebord saillant tuberculeux, et qui est souvent hérissé le long de sa moitié supérieure de très-petites dentelures; ce lobe est presque constamment muni de quatre dents assez longues (du moins dans les individus adultes) le long de sa moitié inférieure. Les bords supérieur et inférieur sont ¿̀ peu près droits et faiblement divergents en avant; les oreillettes cardinales sont bien marquées, l'antúieure porte assez souvent trois dents marginales et un tubercule cardinal plus ou moins luisant, suivant l'état de conservation des valves. La voûte dorsale de ces dernières est plus ou moins fortement carénée le long du côté inférieur; elle est hérissée d'un grand nombre de granulations ou d'épines, plus ou moins nombreuses et fortes. A la surface de la moitié postérieure et surtout le long de la carène, ces épines ou ces granulations sont ordinairement disposées en rangées transversales. La carène est souvent armée de $6-7$ épines assez grosses; elle se termine constanment en arrière par une protubérance obliquement dirigée en dehors et couverte d'un assemblage de petits tubercules, ou surmontée (dans les échantillons Anglais) d'une épine assez forte.

Le tubercule subcentral est fortement développé et obtusement pointu. Immédiatement en arrière de ce tubercule, on remarque un bourrelet noduleux, qui court vers le lobe comprimé postérieur. Le long du bord supérieur, on observe une arête marginale ordinairement épineuse et se terminant brusquement à côté de la protubérance cardinale postérieure.

La surface des valves des jeunes individus est assez grossièrement ponctuée; dans les valves 
des individus plus âgés, elle devient grossièrement réticulée ou rugueuse, et se couvre de tubercules ou d'épines. La carène ventrale et l'arête du côté supérieur des jeunes individus sont ordinairement tranchantes et se terminent postérieurement en une pointe aiguë.

Ma variété A diffère du type de l'espèce par son rebord marginal antérieur couvert de tubercules plus gros et surtout par son arête marginale supérieure, sa carène marginale inférieure et son bourrelet postero-médian, qui sont formés de grosses nodosités et qui ne sont pas couverts de tubercules ou d'épines. L'échantillon du type de l'espèce, figuré pl. IX, fig. $6 a-d$, offre exactement tous les caractères de la Cythere ornatissima du plänermergel de la Bohème, il montre dans son bourrelet postero-médian, ainsi que dans les ornements du côté carénal, un passage bien évident vers la variation de forme representée sur la pl. VII, fig. 7. 1)

La carapace vue par l'extrémité antérieure, offre un aspect tétragonal-quinquelobé. Vue en dessus ou en dessous, elle presente un aspect piriforme ou subpanduriforme dans les jeunes individus. La surface de la région pectorale est couverte de plusieurs séries de tubercules longitudinaux ou obliquement transversaux dans ma variété $\mathrm{A}$. Ces tubercules deviennent béaucoup plus petits vers la ligne de jonction des valves.

Dimensions. - Longueur 1,2 de millimètre, hauteur 0,7 de millimètre et épaisseur 0,6 de millimètre. La variété A n'a que 0,9 de millimètre de longueur, sur 0,5 de millimètre de hauteur' et d'épaisseur.

observations. - Le Dr. Reuss m'ayant communiqué des échantillons Bohémiens de ses Cytherina ornatissima et ciliata, j’ai pu me convaincre que les individus décrits sous ce dermier nom ne sont qu'une simple variation de forme de la première. J'ai pu constater en oûtre que les exemplaires que je viens de décrire du Limbourg, se rapportent exactement à cette même espèce. Je n'aurais certainement pas pu faire ce rapprochement, si je n'eus pu juger que d'après les figures données par l'auteur de la Description des fossiles crétacés de la Bchème. Par des échantillons du gault de l'Angleterre, qui m’ont été communiqués par Mr. JoNes et qui ont été décrits par cet auteur distingué sous le nom de Cythereis ciliata, j’ai pu constater également, que ces derniers ne diffèrent des nôtres et de ceux de la Bohème, que par des granulations moins nombreuses vers le centre des valves, et par les épines carénales plus fortes, d'où vient que ces échantillons Anglais adultes, vus par l'extrémité antérieure, offrent un aspect subtriangulaire.

Bien que je sois disposé à croire avec Mr. Jones, que la Cythere quadrilatera de Mr. Fr. Ad. Roemer soit un jeune âge de l'espèce qui nous occupe, j'ai cru devoir adopter le

1) Cette forme est cependant assez constante dins les couches, dans lesquelles on la rencontre. 
nom qui a été donné par le Prof. Reuss, parceque les figures et la description publiées par l'auteur Hannovrien, me paraissent insuffisantes pour démontrer l'identité, et parceque l'échantillon figuré de Gehrden a été perdu.

Gisement et Localités. - Cette belle Cythere se rencontre dans le Duché de Limbourg, assez fréquemment dans le psammite glauconifère (Système Hervien Dum.) à Slenaken et à Pesaken et dans le même? Système entre Benzenrathhof et Keverberghof près Heerlen. En Belgique elle semble être assez abondante dans la craie blanche sans silex à Heure-le-Romain. On la trouve en Angleterre, suivant Mir. Jones, dans le Greensand de Warmington, dans le gauli de Folkstone et de Leacon-Hill, dans le Chalk-marl de Douvres, dans le detritus de Charing et dans la craie blanche du Sud-Est de l'Angleterre. Elle a été observée par le Prof. Reuss dans le plänermergel de Luschitz, de Rannay, de Kystra et de Brozan et dans le plänerkalk de Kostitz. La variété A n’a été trouvée jusqu’à présent que dans le Système Maestrichtien à Nedercanne, à St. Pierre, à Gronsveld, à Bemelen, à Kunraede et à Bulkomsdal.

35. CYTHERE KONINCKIANA BOSQUET, 1553 .

Pl. IX, fig. $7 a, b, c, d$.

Cypridina Koninchiana Bosquet, 1847. Description des Entomostracés fossiles de la craie de Maestricht. pp. 18, 19, pl. III, fig. $5 a-f$.

Bosquet, 1847. Mémoires de la Société royale des Sciences de Liége. tom. IV, pp. 368,369 , pl. III, fig. $5 a-f$.

Cythereis "Jones, 1849. A Monograph of the Entomostraca of the crelaceous formation of England. Appendix pag. 36.

Cette Cythere a des valves oblongues, tétragono-ellipsoïdales, à bord antérieur obliquément arrondi et marginé d'un limbe aplati très-étroil; terminées en arrière par un lobe comprimé assez large, dont le bord est obliquement arrondi et quadridenté dans sa moitié inférieure; leurs bords pectoral et dorsal sont presque droits. La partie voûtée est tellement convexe dans ses deux tiers postérieurs le long du côté pectoral, que de ce côté elle dépasse le bord valvaire. De ce point elle se ratiache au bord antérieur par une pente assez douce, et rejoint le bord supérieur, ainsi que le lobe comprimé postérieur, par une pente rapide; elle est subcarénée le long du côté pectoral et sa carène peu saillante se termine en arrière par une dent aiguë; l'arête qui forme la limite marginale de la région dorsale se termine aussi postérieurement par une dent semblable, à côté de laquelle on en remarque, dans quelques individus, une seconde. 
Toute la surface du dos des valves est creusée de points nombreux et anguleux. Les tubercules subcentraux, quoique peu saillants, sont bien prononcés, parceque en arrière ils sont le plus souvent limités par un sillon. A côté de l'oreillette cardinale antérieure on remarque une dépression triangulaire oblique.

La région pectorale présente un coutour subsagittiforme et toute sa surface est ornée de points creux oblonģs; la région dorsale est étroite et creusée, à chaque côté de la ligne de jonction des bords valvaires, d'une seule série de points obliques.

Le bouclier présente, vers le tiers postérieur de sa longueur, une section transversale ì contour trigone.

Dimensions. - Longueur 1 millim., hauteur 0,6 de millim. et épaisseur 0,65 de millim.

Rapports et Difrérences. - La Cythere approximata ${ }^{1}$ ) de l'étage Parisien de la ferme de l'Orme etc., quoique très-rapprochée de la Cythere Koninckiana, s'en distingue facilement par son bord antérieur beaucoup moins oblique et garni de 5-6 dents, par son bord inférieur excavé, par la voûte dorsale de ses valves beaucoup moins haute, et par sa carène non terminée par une dent.

Gisement et Localités. - Cette Cythere se rencontre assez fréquemment dans les couches à Bryozoaires du Système Maestrichtien à Nedercanne, à St. Pierre, à Gronsveld, à Keer, à Bemelen, à Geulhem et à Fauquemont; rarement dans ce même Système à Kunraede, ainsi que dans ce même Système et dans le Système Sénonien (calcaire grossier à silex gris) entre St. Pierre et Petit-Lanaye.

36. CYTHERE CELleporacea nov. spec. 1853.

Pl. VII, fig. $\delta a, b, c, d$.

Valves assez larges, oblongues-subtétragonales; à bord antérieur obliquement arrondi et marginé d'un limbe très-étroit, offrant le long de ses deux tiers inférieurs une série de dentelures très-petites; elles sont terminées en arrière par un lobe comprimé subtrigone, quadridenté dans sa moitié inférieure; leur bord dorsal est presque droit é̂ le pectoral présente à chacune de ses deux extrémités un sinus assez profond. Le dos des valves est fortement bombé; au dessus des deux tiers postérieurs du côté inférieur il dépasse même le bord valvaire; il est rattaché au bord supérieur et à la partie comprimée des deux extrémités par une pente rapide. Toute la surface est creusée de poinis très-irrégulièrement anguleux, profonds et disposés con-

1) BosQuet, 1850. Description des Entonostracés fossiles des terrains tertiaires de la France et de la Belgique. pag. 8s, pl. IX, fig. $9 a$-d. 
centriquement autour des tubercules subcentraux. Ceux-ci-sont très-peu proéminents et presque confondus avec le reste de la surface.

La carapace offre une section transversale ì contour arrondi-sub-cordiforme et une section longitudinale à contour ovalaire. La région pectorale, quoique non marginée d'une carène, est nettement limitée et creusée de points irrégulièremént anguleux et peu nombreux. Cette région présente un contour subcordiforme.

Dimensions. - Longueur 1,1 de millimètre, hauteur et épaisseur 0,65 de millimètre.

Kapports et Difrérences. - Elle est voisine de la précédente: elle s'en distingue essentiellement par ses valves plus convexes, plus larges, non carénées, par les dentelures du bord antérieur, par les points creux de la surface et surtout par ceux de la région pectorale beancoup plus grands et d'une forme tout-à-fait différente.

Gisement et Localités. - Elle est beaucoup plus rare que la Cythere Koninckiana, et n'a été trouvée jusqu'à présent que dans les couches à Bryozoaires du Système Maestrichtien à Keer, à Gronsveld et à Geulhem.

\section{Gythere SEmicancellata nov. spec. 1853.}

Pl. VII, fig. $9 a, b, c, d$.

Valves oblongues-subpentagonales; à bord antérieur obliquement sémi-circulaire et marginé d'une partie comprimée, nettement limitée en arrière par une gouttière assez profonde, qui prend naissance immédiatement en arrière de l'oreillette cardinale antérieure; la surface de cette partie comprimée est agréablement ornée de sept fossettes allongées-quadrangulaires. Les valves sont terminées en arrière par un lobe comprimé subtétragone et tourné vers le côté inférieur; leurs bords dorsal et pectoral sont presque droits et faiblement divergents en avant. Le tubercule subcentral est assez gros, oblong et offre à sa surface trois sillons, dont un longitudinal au côté suipérieur, et deux autres, beaucoup plus cours et presque transversaux, au côté inférieur. La partie voûtée, qui est élégamment cancellée dans sa moitié postérieure, est nettement limitée par une arête aiguë le long du côté supérieur; elle est séparée de la région pectorale par une carène surmontée d'une crête simple, inclinée vers le côté pectornl. Le long de cette crête on roit une série de $7-8$ points creux. La région dorsale est élargie en avant et tronquée en arrière; la région pectorale est subcordiforme et offre le long de chacune des deux crêtes carénales un. sillon longitudinal, au fond duquel on voit 3-4 points creux.

La carapace présente vers le tiers postérieur de sa longueur une section transversale à contour deltoïde-subtétragone.

Bimensions. - Longueur 0,6 de millimètre, hauteur et épaisseur 0,35 de millimètre. 
Rapports et Difrérences. - Elle diffère de ma Cythere macropora ${ }^{\mathrm{J}}$ ) des étages 'Tongrien et Parisien supérieur (de laquelle elle se rapproche beaucoup) par ses dimensions plus petites, par la voûte dorsale de ses valves plus haute, par son lobe comprimé postérieur sans dents, par ses tubercules subcentraux non creusés de points quadrangulaires et surtout par le canal profond qui se remarque entre ceux-ci et la partie comprimée antérieure.

Gisement et Localités. - Elle est peu commune et a été trouvée dans la couche à Bryozoaires du terrain Maestrichtien à Nedercanne, à St. Pierre, à Bemelen et à Geulhem. Elle est très-rare dans le même Système crétacé à Kunraede.

38. CYTHERe ORNata Bosquet, 1853.

Pl. IX, fig. $8 a, b, c, d$.

Cypridina ornata Bosquet, 1847. Description des Entomostracés fossiles de la craie de Maestricht. pp. 21, 22, pl. IV, fig. $3 a-f$.

Bosquet, 1847. Mémoires de la Société royale des Sciences de Liége. tom. IV, pp. 371,372, pl. IV, fig. $3 a-f$.

Cythereis "Jones, 1849. A Monograph of the Entomostraca of the cretaceous form. of England. Appendix pag. 36.

Les valves de cette belle Cythere sont allongées-subtétragonales; leur bord antérieur est obliquement sémicirculaire et marginé d'un limbe comprimé lisse et garni d'un grand nombre de petites dentelures marginales presque sétacées; elles sont terminées en arrière par un lobe comprimé assez large, portant 8-9 dents très-pointues, divariquées et beaucoup plus grandes que celles du bord antérieur. Les tubercules subcentraux sont assez gros, saillants et pointus en arrière. Une carène presque droite, surmontée d'une crête assez large, serrulée et se terminant postérieurement en pointe, sépare le dos des valves de la région pectorale. Toute la surface de la partie voûtée et du lobe comprimé postérieur est creusée de points arrondis ou parfois anguleux; dans sa moitié postérieure elle est divisée en deux parties presque également larges par un sillon longitudinal; la partie supérieure constitue un lobe, garni d'environ 7 dents marginales aiguës, assez longues et acuminées. L'oreillette cardinale antérieure est échancrée au milieu. Immédiatement en arrière de cette oreillette, on remarque une dépression triangulaire oblique. La région dorsale offre quatre séries longitudinales de points creux; la région pectorale

1) Boseutet, 1550. Description des Entomostracés fossiles des terrains tertiaires de la France et de la Belgitue. pag. 47, pl. V, fig. $2 a-d$. 
est triangulaire-subsagittiforme et creusée, le long de chacune des deux carènes, d'une série de points assez profonds.

Le bouclier présente vers le tiers postérieur de sa longueur un contour deltoïdal.

Les valves ont parfois conservé des restes de la couleur qu'elles avaient pendant la vie de l'animal et sont alors d'un rose-purpurin.

Dimensions. - Longueur 1 millimètre, hauteur et épaisseur 0,6 de millimètre.

uapports et Difrérences. - La Cythere (Crpridina) Hatdingeri Reuss ${ }^{1}$ ) spec。 du leithakalk de Nussdorf près Vienne et des étages Tongrien, Parisien et Suessonien de la France, a quelques rapports avec la Cythere ornata. L'espèce tertiaire s'en distingue néanmoins bien nettement, par sa carène et son arête dorsale non dentelées, par son lobe comprimé postérieur garni seulement de cinq dents rapprochées, et par ses tubercules subcentraux non pointus.

Gisement et Localicés. - Cette espèce n'est pas rare dans le Système Maestrichtien à Nedercanne, à St. Pierre, à Gronsveld, à Bemelen, à Keer, à Geulhem, à Fauquemont et à Kunraede. Elle se rencontre aussi, mais rarement, dans le calcaire grossier à silex gris du Système Sénonien entre St. Pierre et Pelit-Lanaye, et dans une couche qui parait appartenir au Système Hervien entre Benzenrathhof et Keverberghof près Heerlen. En Belgique on la trouve dans le terrain Maestrichtien ì Wonck et à Sichen (Limbourg) et à Ciply piès Mons, ainsi que dans le calcaire grossier à silex gris du Système Sénonien ì Frère près Tongres.

39. cythere serrulata Bosquet, 1853.

Pl. IX, fig. $9 a, b, c, d$.

Cytherina cornuta? Reuss, 1846. Versteinerungen der Böhmischen Kreide-formation. Zweite Abtheil., pag. 105 , pl. XXIV, fig. $20 a, b, c$, (non Fr. AD. Roemer). Cypridina serrulata Boseuet, 1847. Descript. des Entomostr. foss. de la craie de Maesiricht. pag. 20, pl. IV, fig. $2 \alpha-d$.

Bosquet, 184\%. Mémoires de la Société Royale des Sciences de Liége. tom. IV, pag. 370, pl. IV, fig. $2 a-d$.

Cythereis cornuta Jones, 1849. A Monograph of the Entomostr. of the cretaceous formation of England. Appendix pag. 36, (non Cytherina cornuta Fr. AD. ROEMER).

1) Meuss, 1849. Die fossilen Entonostracéen des Oesterreichischen tertiärleckens. pag. 38, pl. X, fig. $13 a, b$. Bosquet, 1850. Description des Entomostracés fossites des terrains tertiaires de la France et de la Belgique. pag. $125, \mathrm{pl}$. VI, fig. $10 a-e$. 
Cypridina cornuta Reuss apud Gernitz, 1850. Das Quadersandstein-gebirge oder Kreidegebirge in Deutschland. pag. 100.

Valves allongées, obscurément tétragonales; à bord antérieur obliquement sémicirculaire, et muni dans sa moitié inférieure de 5-6 dents larges et tronquées au sommet; elles sont terminées en arrière par un lobe comprimé assez large, de forme tétragonale et garni de cinq dents rapprochées et tronquées comme celles du rebord marginal antérieur; leurs bords inférieur et supérieur sont presque droits et très-peu divergents en avant; le dernier offre antérieurement une oreillette peu saillante, et postérieurement une dent aiguë. Ia partie voûtée est carénée, et la carène est surmontée d'une crête serrulée et terminée en arrière en pointe; à côté de cette pointe on voit une dent, pareille à celle qui se trouve au côté supérieur sur l'oreillette cardinale postérieure. Toute la surface est lisse et luisante. Les tubercules subcentraux sont ovalaires et mucronés en arrière. La région pectorale est aplatie, sagittiforme et creusée, le long de chacune des deux carènes, d'une série de points.

La carapace présente, vers le tiers postérieur de sa longueur, un contour deltoïdal, dont les trois côtés sont faiblement excavés.

Cette espèce a parfois conservé des restes de ses couleurs; ses valves sont alors d'un rouge incarnat sur les bords antérieur, postérieur et inférieur.

Dimensions. - Longueur 1,1 de millim., hauteur 0,55 de millim. et épaisseur 0,8 de millim.

observations. - Il serait bien possible que la Cythere (Crtherina) cornuta Reuss, du plänermergel de Luschitz, fût la même que ma Cythere serrulata. La figure citée et la description donnée par l'auteur de Bilin, me paraissent cependant insuffisantes pour que l'on puisse décider cette question. Pour ce qui concerne l'espèce décrite par Mr. R.•Jones, sous le nom de Cythere (Crтhereis) cornuta, je puis affirmer, non seulement d'après les figures et la description qui en ont été données par l'auteur Anglais, mais encore d'après les échantillons même que je dois à son obligeance, que cette espèce en est toute différente, et ne peut être identifiée, ni à ma Cythere serrulata, ni à la Cythere cornuta RoEmer.

Eapports et minérences. - La Cythere (Crtherina) cornuta Roemer ${ }^{1}$ ) de l'étage Parisien est bien certainement voisine de la serrulata. La cornuta cependant n'a jamais la crête de sa carène serrulée dans toute sa longueur, elle n'offre jamais de dent ì côté du tubercule

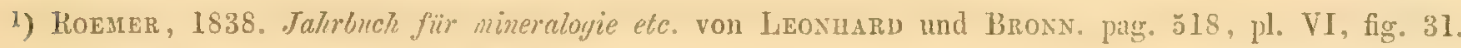
Boseuet, 185u. Description des Enlomostracés fossiles des terrains tertiaires de la France et de la Betyique. pag. 117, pl. VI, fig. $4 a--d$. 
cardinal postérieur, et à côté de la corne ou pointe, qui termine la crête carénale, elle n’a point d'oreillette cardinale; enfin, elle n'offre point, comme la Cythere serrulata, sur sa région pectorale une série de points creux, le long de chacune des deux crêtes carénales.

Gisement et Localités. - Elle est rare et se rencontre dans le Système Maestrichtien à St. Pierre et à Kunraede, et dans le calcaire grossier à silex gris, du Système Sénonien, entre St. Pierre et Petit-Lanaye, et dans le Système Hervien? entre Benzenrathhof et Keverberghof près Heerlen. En Belgique elle se trouve dans le Système Maestrichtien à Sichen, à Folx-les-Caves et à Ciply près Mons. Dans cette dernière localité elle se rencontre aussi dans le calcaire grossier à silex gris du Système Sénonien.

40. Cythere Phylloptera nov. spec. 18503.

Pl. VII, fig. $10 a, b, c, d$.

Cette singulière espèce a des valves allongées-tétragonales; à bord antérieur obliquement sub-sémicirculaire et marginé d'un rebord saillant, garni sur ses deux tiers inférieurs de plusieurs dents tronquées; terminées en arrière par une partie comprimée large, obliquement tétragone, marginée et munie de $6-7$ dents assez longues, rapprochées et tournées vers le côté pectoral; leurs bords supérieur et inférieur sont presque droits et très-faiblement divergents en avant. Le bord supérieur présente près de son extrémité antérieure une épine assez longue, vers le tiers postérieur de sa longueur une lame quadrangulaire redressée, et à son extrémité postérieure une pointe saillante, surmontant la protubérance cardinale. Le bord inférieur est marginé d'une crête lamelleuse, et à côté du tiers postérieur de la longueur de ce bord, la partie voûtée, qui est lisse et luisante dans toute son étendue, s'élève brusquement en une aile très-haute, bidentée en avant et en arriére.

La région pectorale a la forme d'une croix, dont les deux branches latérales offrent l'aspect d'une feuille bidentée à chacun de ses bords. La carapace vue par l'extrémité antérieure, présente un aspect triangulaire, à angles latéraux aigus et à côtés latéraux concaves.

Dimensions. - Longueur 0,8 de millimètre, hauteur 0,45 de millimètre et épaisseur 0,55 de millimètre avec l'aile.

Rapports et Dirférences. - Quoique la Cythere ceratoptera ${ }^{1}$ ) du Système Rupélien de Bazele près Rupelmonde et de Bergh, en Belgique, se rapproche de celle-ci par la forme et par plusieurs autres caractères, elle s'en distingue cependant nettement par sa crête carénale

1) Bosquet, 1850. Description des Entomostracés fossiles des terrains tertiaires de la France et de la Belgique. pp. $114,115, \mathrm{pl}$. VI, fig. $2 a-d$. 
surmontée d'un très-grand nombre d'épines aplaties et bifides au sommet et par son arête marginale supérieure, garnie d'un grand nombre de dentelures aiguës.

Gisement et Localités. - Elle est très-rare; je n’en ai trouvé que deux échantillons. dont une valve gauche dans le calcaire grossier à silex gris à St. Pierre (Duché de Limbourg) et un échantillon bivalve en Belgique dans la même couche crétacée de Ciply près Mons.

41. cythere alata Bosquet 1853.

Pl. IX, fig. $10 a, b, c, d$.

Cypridina alata Bosquet, 1847. Description des Entomostracés fossiles de la craie de Maestricht. pp. 19, 20, pl. IV, fig. $1 a-d$.

" "Bosquet, 1847. Mémoires de la Société royale des Sciences de Liége. tom. IV, pp. 369,370 , pl. IV, fig. $1 a-d$.

Cythereis "Jones, 1849. A MTonograple of the Entomostraca of the cretaceous formation of England. Appendix pag. 36.

" " ? Jones, 1849. Ibidem. tom. V, pp. 21, 22, fig. 14a-d.

Valves ovales-subtrigones; comprimées en avant; à bord antérieur très-obliquement subtronqué; terminées en arrière par un lobe comprimé, dont le bord est arrondi et garni de trois dents très-petites et très-rapprochées, les bords inférieur et supérieur sont presque droits et assez fortement divcrgents en avant; le dernier offre à son extrémité antérieure une oreillette assez grande. La partie voûtée des valves est fortement carénée le long du côté pectoral, et la carène est surmontée d'une aile terminée en arrière par une pointe aiguë. Le long de l'aile carénale on remarque deux à trois points creux allongés. Les tubercules subcentraux sont trèspeu marqués. La région pectorale est aplatie, large, et présente un contour hasté-subsagitté. La carapace offre, vers le tiers postérieur de sa longueur, une section transversale à contour triangulaire, dont deux côtés sont concaves et dont les deux angles latéraux sont très-aigus.

Le bord antérieur seul est parfois d'un beau jaune-orangé, tandis que le reste de la surface est d'un blanc-laiteux.

Dimensions. - Longueur 1,10 de millimètre, hauteur 0,7 de millimètre et épaisseur 1,2 de millimètre avec l'aile carénale.

Rapports et Dimérences. - Mr. Jones a trouvé dans le Detritus de Charing et dans la craie blanche de Norwich, de Gravesend et de Charlton une espèce qu'il a cru être la même que ma Cythere alata des environs de Maestricht, et qu'il a décrite sous le même nom spécifique. Cette espèce me semble différente de la mienne. D’après la figure donnée par Mr. 
JoNEs, les valves de l'espèce Anglaise sont beaucoup plus larges en arrière, moins obliquement arrondies en avant, leur oreillette cardinale antérieure est moins saillante, leur lobe comprimé postérieur est beaucoup plus large et garni de dents beaucouq plus fortes. Les valves offrent en oûtre au bord antérieur de grosses dents, dont je ne puis trouver la moindre trace dans les échantillons de ma Cythere alata.

Gisement et Localités. - - Cette interessante espèce se trouve dans le Système Maestrichtien à Kunraede et dans ce même Système, ainsi que dans le calcaire grossier à silex gris du Système Sénonien, entre St. Pierre et Petit-Lanaye. En Belgique elle se rencontre dans le Système Maestrichtien de Sichen (Limbourg). Elle semble être rare partout.

42. CYTHERE LATicristata nov. spec. 1853.

Pl. VII, fig. $11 a, b, c, d$.

Valves à contour ovale-subtrigone; comprimées aux deux extrémités; à bord antérieur obliquement sémicirculaire, à·bord postérieur arrondi et muni de quatre dents extrémement courtes, presque imperceptibles; les bords inférieur et supérieur sont presque droits et divergents en avant. La voûte dorsale est lisse dans toute son étendue; elle est fortement carénée le long du côté inférieur, et sur sa carène s'élève une crête très-large, tellement inclinée vers le côté pectoral, qu'elle dépasse de beancoup le bord valvaire. La crête carénale est faiblement arquée et son extrémité postérieure obliquement tronquée. Le long de la base de cette crête, on remarque trois points creux obsolètes.

Le bouclier offre, vers le tiers postérieur de sa longueur, une section transversale à contour triangulaire, dont les côtés sont concaves et dont les angles latéraux sont aigus. La région pectorale présente un contour hasté.

Dimensions. - Longueur 0,9 de millimètre, hauteur 0,6 de millimètre et épaisseur 0,75 de millimitre.

Rapports et Bifférences. - Elle diffère essentiellement de la précédente, par ses valves beaucoup plus larges, ̀̀ bord antérieur beaucoup moins obliquement arrondi et sans oreillette cardinale antérieure. La crête carénale n'est pas terminée par une pointe-aiguë; le bord de cette crête forme au contraire avec la troncature postérieure un angle obtus.

Gisement et Localités. - Elle est très-rare dans le Duché de Limbourg et se trouve dans la craie blanche sans silex (Système Sénonien Dumont) à Slenaken; dans le Système Hervien à Slenaken, à Pesaken, à Simpelveld et entre Benzenrathhof et Keverberghof près Heerlen. En Belgique elle parait aussi être assez rare dans le calcaire grossier à silex gris à Ciply près Mons, et au contraire assez fréquente dans la craie blanche sans silex à Heure-le-Romain. 
43. CYTHERE TRIGONOPTERA. nov. spec, 1853.

Pl. VII, fig. $12 a, b, c, d$.

Cette Cythere a des valves dont le contour est sensiblement pentagonal. Ces valves sont comprimées aux deux extrémités; leur bord antérieur est sémicirculaire et le postérieur angnleux, ou plutôt terminé en une pointe courte; leur bord supérieur est droit, tandis que l'inférieur est fortement arqué. La partie voûtée est lisse et luisante, mais offre, vers le tiers postérieur de sa longueur, une carène, surmontée d'une aile trigone et très-haute. La région pectorale présente un contour tétragonal-hasté. Le bouclier offre, vers le tiers postérieur de sa longueur, une section transversale à contour triangulaire, dont les deux côtés latéraux sont faiblement concaves dans leur moitié inférieure, et dont les deux angles latéraux sont aigus.

Dimensions. - Longueur 0,6 de millimètre, hauteur 0,35 de millimètre et épaisseur 0,46 de millimètre.

Hapports et Diférences. - Elle a les plus grands rapports avec la Cythere (Crpridira) vespertilio Reuss, ${ }^{1}$ ) du tegel de Grinzing près Vienne. L'espèce tertiaire Autrichienne diffère cependant de mon espèce crétacée, par son bord supérieur arqué, par son bord inférieur droit, par la pointe de sa partie comprimée postérieure plus rapprochée du côté pectoral, par sa crête carénale plus grande et plus large, ${ }^{2}$ ) et par la dent que présente, sur chaque valve, la troncature postérieure de cette crête.

Gisement et Localités. - Bemelen et Geulhem, dans le Système Maestrichtien. Thès-rare.

44. GYTHERE MiNUTA nov. spec. 1853.

Pl. X, fig. I $a, b, c, d$.

Valves oblongues-tétragonales; à bord antérieur obliquement sémi-circulaire et marginé d'une partie comprimée large, creusée d'une seul sillon et garnie vers le quart inférieur de sa hanteur de deux petites dents rapprochées. Ces valves sont terminées en arrière par une lobe comprimé subtrigone. Leurs bords supérieur et inférieur sont presque droits et parallèles. Le premier offre en avant une oreillette saillante et en arrière un angle, qui termine l'arête marginale de la région dorsale. Leur partie voûtée est le plus haute vers le tiers postérieur de la longueur; elle se rattache au bord supérieur et à la partic comprimée antérieure par une pente assez

1) Ruuss, 1849. Die fossilen Entomostr. des Oesterr. tertiürbeckens. tom. XI, pag. 41, fig. 10 a-c.

2) Le bord de la crête carénale forme avec la troncature postérieure un angle trìs-aigur. Dans ma Cythere trigonoptera l'angle formé de la même manière est presque droit. 
douce, elle rejoint le lobe comprimé postérieur par une pente rapide et retombe perpendiculairement sur le bord inférieur; toute la surface est lisse; le tubercule subcentral est saillant, oblong et arrondi en arrière. La région pectorale est plane et son contour est hasté-subtrigone. La carapace offre une section transversale à contour tétragone.

Dimensions. - Longueur 0,5 de millimètre, hauteur et épaisseur 0,3 de millimètre.

izapports et Différences. - Ma Cythere pusilla ${ }^{1}$ ) de l'étage Falunien des environs de Bordeaux, se rapproche de celle-ci. L’espèce tertiaire cependant en diffère, par le bord antérieur de ses valves largement arrondi et sans dents, par sa crête carénale moins pointue en arrière, par sa voûte dorsale offrant deux petites côtes longitudinales et une série de tubercules oblongs à la base de la crête carénale, et enfin par le sillon profond au côté pectoral de la moitié antérieure de cette dernière.

Gisement et Localités. - Fille est rare dans les couches à Bryozoaires du Système Maestrichtien et n'a été trouvée qu’à Nedercanne et à Bemelen.

45. CYTHERE HaGenowi nov. spec. 1853.

Pl. X, fig. $2 a, b, c, d$.

Les valves de cette Cythere sont oblongues-tétragonales; leur bord antérieur est obliquement subsémicirculaire et marginé d'un limbe comprimé étroit et lisse; elles sont terminées en arrière par un lobe comprimé, fortement tourné vers le côté inférieur et muni de trois petites dents; les bords inférieur et supérieur sont presque droits et parallèles. Leur partie voûtée est très-haute dans ses deux tiers postérieurs; elle se rattache à la partie comprimée antérieure et au bord supérieur par une pente assez rapide, elle rejoint le lobe comprimé postérieur par une pente très-rapicle et retombe perpendiculairement sur le bord inférieur; le long de ce dernier côté elle est fortement carénée, et sa carène est surmontée d'une crête arquée, très-élevée, dont le bord est aigu, faiblement plissé; et qui se termine brusquement en arrière en une pointe émoussée. Le long de cette crête on remarque 8 fossettes, qui s'étendent presque jusqu’à ses bords et qui, dans un assez grand nombre d'individus, sont remplacées par des trous. Alors toute la crête est perforée à sa base. Le long de la partie comprimée antérieure on observe une seule série de points creux, parallèle au bord. Les tubercules subcentraux sont assez grands, mais peu proéminents. Toute la surface de la partie voûtée des valves est creusée d'un trèsgrand nombre de points, superficiels sur la moitié antérieure, très-bien marqués sur la moitié

1) Bosquet, 1850. Description des Entomostracés fossiles des terrains tertiaires de la France et de la Belgique. pp. 85,86, pl. IV, fig. $7 a-d$. 
postérieure et disposés par séries transversales arquées, partant de la base de la crête carénale et allant se terminer sur la région dorsale. Immédiatement en arrière du tubercule cardinal antérieur, on remarque une dépression triangulaire oblique. La région pectorale, qui est plane, et qui offre un contour sub-cordiforme-hasté, est creusée de plusieurs séries de points, pareils à ceux qui recouvrent le dos des valves, et le long de la crête carénale, on voit 8 fossettes profondes, placées au fond de sillons transversaux. Ces 8 fossettes correspondent exactement à celles du dos des valves.

La carapace présente une section transversale à contour deltoïdal.

Dimensions. - Longueur 0,65 de millim., hauteur 0,35 et épaisseur 0,4 de millim.

Hapports es Diférences. - La Cythere Forbesiana 1) des étages Parisien et Suessonien des environs de Paris a de très-grands rapports avec celle-ci. L'espèce tertiaire s'en distingue cependant par ses valves marginées antérieurement d'un limbe plus large et creusé de deux sillons parallèles au bord, par le lobe comprimé postérieur non garni de dents, par les points creux de la surface moins nombreux et non disposés par séries transversales, et par la forme de la région dorsale.

Gisement et Localités. - Cette Cythere est peu commune dans le Système Maestrichtỉen à Nedercanne, à St. Pierre, à Gronsveld, à Bemelen, à Keer, à Geulhem, à Fauquemont et à Vieux-Fauquemont.

46. Cythere machoptera nov. spec, 1853.

Pl. X, fig. $3 a, b, c, d$.

Valves oblongues-subpentagonales; à bord antérieur obliquement sémicirculaire, et nırginé d'un limbe comprimé, dont la surface est creusée de cinq fossettes tétragones assez profondes; elles sont terminées en arrière par un lobe comprimé subtrigone, muni à son côté inférieur d'une dent unique; les bords supérieur et inférieur sont presque droits. Le dos des valves est séparé de la region pectorale par une carène, surmontée d'une crête très large et fortement tournée vers le côté pectoral; cette crête est tronquée en arrière et terminée en pointe; sa surface est creusée de $7-8$ lignes obliques, partant d'un nombre égal de points creux arrondis, qui se trouvent le long de sa base. Les tubercules subcentraux sont grands, arrondis en arrière et peu proéminents. Entre ces tubercules et le lobe comprimé postérieur on remarque deux côtes longitudinales droites et parallèles. Immédiatement en arrière du tubercule cardinal anté-

1) Bosquet, 1850. Description des Entomostracés fossiles des terrains tertiaires de la l'rance et de la Zelgique. pp. 129,130, pl. VI, fig. $13 a-d$. 
rieur, on voit une dépression triangulaire oblique; à coté de l'extrémité postérieure de l'arête marginale supérieure on observe trois points creux, plus ou moins trigones et inégaux. 'Toute la surface de la partie voûtée et creusée de points très-petits.

La région pectorale est très-large; elle présente un contour hasté et offre le long de chacune des deux crêtes carénales, une série de points creux, placés au fond de sillons en forme de V renversé. Près de son extrémité postérieure, à côté de la ligne de jonction des bords valvaires, cette région présente encore trois points creux, semblables à creux qui se trouvent sur le dos de chaque valve, à côté du tubercule cardinal postérieur.

Le bouclier offre, vers le tiers postérieur de sa longueur, une section transversale à contour subtriangulaire.

Dimensions. - Longueur 0,65 de millim., hauteur 0,4 de millim. et épaisseur 0,55 de millim.

mapports et miférences. - Elle se distingue au premier coup-d'oeil de la précédente, par sa partie comprimée antérieure creusée de grands points quadrangulaires, par sa crête carénale plus haute, par son lobe comprimé postérieur large et beaucoup moins fortement tourné vers le côté pectoral et enfin par les deux côtes longitudinales droites, qui s'élèvent sur la moitié postérieure de sa partie voûtée.

Gisement et Localités. - Cette singulière espèce est fort rare et n'a été trouvée qu'à Bemelen dans le Système Maestrichtien.

47. GXTHERE CRISTATA nov. spec. 1853.

PI. $\mathrm{X}$, fig. $4 a, b, c, d$.

Valves allongées, à contour trapézoïdal; à bord antéricur obliquement subsémicirculaire et marginé d'un limbe comprimé, creusé d'un seul sillon parallèle au bord; terminées en arrière par un lobe comprimé tridenté et fortement tourné vers le côté inférieur. Leurs bords supérieur et inférieur sont presque droits et parallèles. Leur partie voûtée est séparée de la région pectorale par une carène, surmontće d'une crête dentelée et faiblement arquée. Le long de la base de cette crête on remarque 8 fossettes, d'oú partent des sillons, qui vont abouter à ses 8 dents marginales. Les tubercules subcentraux sont grands, peu proéminents, mais nettement limités en arrière; de leur partie postérieure partent deux gros plis onduleux, qui se dirigent vers deux des quatre points creux, que l'on remarque en arrière du limbe antérieur. Tout le reste de la voûte dorsale des valves, est orné de rides transversales noduleuses irrégulières. Deux de ces rides, produisent sur l'arête marginale de la région dorsale, des saillies assez fortes. L'oreillette cardinale antérieure est émarginée à son bord, et le tubercule cardinal antérieur très-bien prononcé; en arrière de celui-ci on remarque une dépression triangulaire, et à son côté inférieur, 
trois petits tubercules. La région pectorale présente un contour hasté-subsagitté; sa surface est élégamment ornée de quelques protubérances, et le long de chacune des deux crêtes carénales, 'elle est creusée d'une série de points, d'oú partent des plis qui vont aboutir aux dentelures marginales. La carapace offre, vers le tiers postérieur de sa longueur, une section transversale à contour deltoïdal.

Dimensions. - Longueur 0,7 de millim., hauteur 0,4 de millim. et épaisseur 0,5 de millim.

IRapports et Différences. - Cette Cythere est très-remarquable et ne saurait être confondue avec aucune de ses congénères connues.

Fisement et Localités. - La Cythere cristata est une des plus belles du Genre; elle se rencontre dans le Système Maestrichtien à Nedercanne, à St. Pierre, à Gronsveld, à Bemelen, ì Keer, à Geulhem et à Fauquemont; et dans le calcaire grossier à silex gris du Système Sénonien entre St. Pierre et Petit-Lanaye.

Gen. V. CTPPELI DE Koninck, 1841.

Lynceus? Mü̈LER, 1785. Entomostraca sen insecta teslacea, quae in aquis Daniae et Norvegiae reperit. pp. 67 et suivantes.

Cyprella de Koninck, 1841. Mémoire sur les Crustacés fossiles de Belgique. pag. 19.

DE Koninck, 1844. Description des animaux fossiles du terrain carbonifère de Belgigue. pp. $589,590$.

Daphnia M'cox, 1844. Synops. of the Charact. of the Carboniferous limestone fossits of Ireland. Cyprella Bosquet, 1847. Mémoires de la Société royale des Sciences de Liége. tom. IV, pp. $372,373$.

Boseuet, 1847. Description des Entomostracés fossiles de la craie de Maestricht. pp. 22, 23.

Bronn, 1848. Index Palaeontologicus. Oder Uebersicht der bis jetzt bekannten fossilen Organismen. pag. 385.

Cypridina Jones, 1849. A Monograph of the Entomostraca of the cretaceons formation of England. pag. 5 and pag. 36. (non M. Edwards).

Cyprella Bosouet, 1850. Description des Entomostracés fossiles des terrains tertiaires de la France et de la Belgique. pp. 130-132.

Caractères Génériques. - Carapace bivalve, formée de deux couches distinctes; acuminée en arriére et munie en avant d'un prolongement en forme de bec, présentant une ouverture triangulaire qui est tournée vers le côté pectoral. Deux tubercules subcentraux, ou du moins 
deux grandes fossettes internes arrondies et situées un peu en avant de la partie moyenne de chaque valve. ${ }^{1}$ )

Le bord supérieur interne des valves et élargi et muni d'une charnière dorsale, formée sur la valve droite de deux dents, l'une triangulaire oblique, située au milieu de ce bord, l'autre tout-à-fait postérieure et quadrangulaire. Ces deux dents sont reçues dans deux fossettes de la valve opposée. Le bord supérieur de la valve gauche est atténué et s'engage sous le bord de la valve correspondante; inférieurement, le bord de la valve gauche s'engage au contraire dans un sillon, du bord élargi de l'autre valve.

Contrairement à l'avis de Mr. Jones, je ne pense pas que le genre Cyprella puisse être régardé comme le même que le genre Cypridina. D’après les figures données par Mr. Mrune Enwards de la Cypridina Raynaudii, 2) qui a servi de type, les valves dans ce dernier genre sont dépourvues de rostre et d'une ouverture triangulaire ou sémilunaire au bord antérieur.

Le genre Cyprella est très-voisin du genre Lynceus de MüLLter; on n’en connait jusqu’à présent que cinq espèces, dont une tertiaire du bassin Parisien, deux des couches Palaeozoïques, l'une signalée par Mr. M'Coy dans le calcaire carbonifère de l'Trlande, et l'autre par Mr. le Professeur De Konnck dans celui de Visé en Belgique. Les deux autres proviennent du terrain crétacé et ont déja été signalées par moi en 1847. Ces espèces sont:

1. Cyprella ovulata Bosquet, $184 \%$.

Pl. IX, fig. $11, a, b, c$.

Cyprella ovulata Bosquet, 1847. Descr. des Entomostracés fossiles de la craie de Maestricht. pag. $23, \mathrm{pl}$. IV, fig. $4 a-c$.

" Bosquet, 1847. Memoires de la Soc. royale des Sciences de Liége. tom. IV, pag. $373, \mathrm{pl}$. IV, fig. $4 a-c$.

Cypridina "Jones, 1849. A Monograph of the Entomostraca of the cretaceous formation of England. Appendix pag. 36.

Valves ovalaires, fortement bombées, munies antérieurement d'un rostre assez court et

1) La fossette interne de chaque valve des Cyprella présente une différence notable d'avec celle des Cythere, des Cytheridea et des Bairdia. Cette différence consiste en ce que les points creux disposés par séries flexueuses, que l'on remarque chez elles au fond de cette fossette, traversent en entier la couche interne, (ou même les deux couches dont se composent les valves).

2) Voyez, Mrtne EdWards, 1840. Histoire Naturelle des Crustacés (dans Suites à Burfon) pl. 36, fig. 5. 
terminées en arrière en une pointe très-courte. Leur surface est recouverte d'un grand nombre de petites excavations arrondies, peu profondes, d'autant plus petites qu'elles sont plus éloignées de la partie centrale des valves, et disparaissant entièrement vers les bords.

La place du tubercule subcentral est orbiculaire et creusée d'un grand nombre de points allongés, semblables à des piqûres et disposés par lignes flexueuses et anguleuses.

Dimensions. - Longueur 0,2 millimètres, hauteur 1,4 de millimètre et épaisseur 1,2 de millimètre.

Rapports et Difrérences. - Elle differe de la Cyprella Koninckiana, par sa taille plus grande, par la forme ovalaire de ses valves, par les points creux de sa surface grands et arrondis, et par sa pointe antérieure constamment plus courte.

Gisement et Localités. - Cette belle Cyprella est très-fragile et extrémement rare dans le calcaire grossier à silex gris entre St. Pierre et Petit Lanaye, dans le Duché de Limbourg. Elle se rencontre aussi en Belgique dans le Système Maestrichtien à Sichen.

2. GYPRELLA KONINGKIANA BosQUET, $184 \%$.

Pl. IX, fig. $1: 2, b, c$.

Cyprella Koninckiana Bosovet, 1847. Description des Entomostracés fossiles de la craie de Maestricht. pp. 23, 24, pl. IV, fig. $5 a-c$.

Bosquet, 1847. Mémoires de la Société royale des Sciences de Liége. tom. IV, pp. 373,374 , pl. IV, fig. $5 a-c$.

Cypridina "J Jones, 1849. A Monograph of the Entomostraca of the cretaceous form. of England. Appendix pag. 36.

Les valves de cette Cyprella sont subovales; leur prolongement antérieur est aigu et plus grand que celui de l'espèce précédente, et la pointe, par laquelle elles se terminent en arrière, est acuminée; leur bord supérieur est faiblement sinué vers son tiers postérieur, tandis que le bord inférieur offre un léger sinus vers le tiers antérieur de sa longueur. La place du tubercule central est recouverte, comme dans l'espèce précédente, d'un grand nombre de points creux oblongs, semblables à des piqûres d'une pointe de canif et disposés en lignes flexueuses et anguleuses.

La surface de la moitié postérieure des valves est creusée de points oblongs très-nombreux, disposés par séries flexueuses et rayonnantes vers les bords, sur lesquels ils disparaissent.

Dimensions. - Elle est constamment plus petite que la Cyprella ovulata, et n'a que 1,2 de millimètre de longueur, sur 0,7 de millimètre de hauteur. 
IRapports et Différences. - La Cyprella Edwardsiana 1) de l'étage Parisien de la France, est voisine de celle-ci. L'espèce tertiaire en diffère cependant par les bords supérieur et inférieur de ses valves non sinués, par la voûte dorsale de ses valves plus fortement bombée et recouverte dans toute son étendue de points creux plus espacés et non allongés.

Gisement et Localites. - Elle se rencontre dans les couches à Bryozoaires du Système Maestrichtien à St. Pierre, à Gronsveld, à Keer et à Bemelen. En Belgique, elle se trouve dans le même Système crétacé à Petit-Lanaye et à Sichen.

1) Bosquet, 1850. Description des Entomostr. fossiles des terrains tertiaires de la France et de la Belgique. pp. 132, 133, pl. VI, fig. 14a-d. 


\title{
C. M A L A C O S T R A C A, MürLer.
}

\author{
DECAPODA, Latreili,e.
}

( $*$ MACRURA.

1. ASTACINA.

Gen. I. ovcoparex nov. gen. 1853.

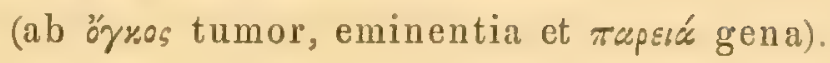

Caractères Géneriques. - Céphalothorax cylindrique, garni de petites granulations et prolongé antérieurement en un rostre trigone, qui est armé sur chacun de ses bords latéraux supérieurs de dents spiniformes assez longues. La hauteur du céphalothorax n'égale pas tout-ì-fait la moitié de la longueur, quand on ne fait pas abstraction du prolongement antérieur. Il est divisé par un sillon assez profond en deux parties principales, une antérieure qui, avec le rostre, occupe presque les deux tiers de la ligne dorsale médiane, et une postérieure, qui est obscurement partagée en trois régions par deux sillons larges, mais peu profonds et n'atteignant pas le rebord marginal postérieur. La partie principale antérieure (la région stomacale) offre de chaque côté un sillon en forme de Y renversé, dont l'axe, qui est tourné en haut, est à peu près parallèle au grand sillon transversal, et dont les deux branches latérales embrassent une petite région proéminente trigone et reposant sur le prolongement inférieur du grand sillon. Celui-ci n'atteint pas le bord inférieur du céphalothorax et va se terminer vers la moitié de la hauteur du bord antérieur; ce dernier bord offre, entre ce même endroit et l'entaille oculaire un prolongement court et obtus, à côté duquel on remarque un gros tubercule. Un peu en arrière de celui-ci et à peu près sur la même ligne longitudinale, on voit un tubercule pareil, mais un peu plus petit. Au dessus du premier de ces tubercules, à côté de la base des bords latéraux du rostre, on observe un troisième tubercule analogue. L'entaille du céphalothorax, servant à la réception de l'abdomen, est assez profonde; elle est bordée d'un limbe marginal qui s'élargit beaucoup, et qui devient anguleux au milieu dans ses deux tiers latéraux.

D'après des échantillons de la collection du Dr. VAN BrEDA, et du Musée Géologique de Harlem, l'abdomen se compose du nombre normal de sept segments, et sa longueur totale est plus grande que celle du céphalothorax avec son rostre. Le premier article est le plus court, le 
second est plus long que les suivants, excepté le pénultième qui le surpasse un tant soit peu en longueur. La longueur du $3^{\text {me }}$ segment est, à très peu de chose près, égale à celles des $4^{\text {me }}$ et $5^{\mathrm{me}}$. Ces trois derniers segments, ainsi que le premier et le second, présentent des prolongements latéraux lamelleux, ou des lobes, qui ont beaucoup de ressemblance avec ceux de la Nephrops Norwegica. La nageoire caudale me semble aussi avoir également beaucoup de rapports avec la même partie dans le genre Nephrops.

Les supports oculaires sont très-gros et semilunaires?

Des pattes je ne connais jusqu'ici que la main, avec des fragments et des empreintes incomplètes des doigts. Ces derniers sont très-longs et déprimés; ils sont garnis, (comme la portion palmaire de la main), sur leur bord interne, d'une rangée de tubercules spiniformes. D'après le petit nombre d'échantillons que j'ai eu occasion de voir, la main droite est plus forte que la main gauche. Dans un échantillon de la collection du Dr. VAN BrEDa, je n’ai pu . observer que quelques fragments des pattes postérieures du côté gauche; dans un échantillon du MLsée Géologique de Harlem on remarque deux articles d'une des pattes postérieures du côté droit; à savoir le second et le $3^{\text {ne }}$ article, qui sont longs et assez grèles.

IRapports et Diférences. - Le genre Oncopareia se rapproche beaucoup du genre Hoplopareia (ia). Mr. M’Cor, qui a créé ce dernier genre, en a fait connaître deux espèces du terrain crétacé de l'Angleterre, et deux autres du London-clay. Le genre Hoplopareia se distingue. bien nettement du nôtre, par les $3^{\text {me }}, 4^{\text {me }}$ et $5^{\text {me }}$ segments de l'abdomen, qui se terminent de chaque côté par une dent pointue, ainsi que par la disposition des sillons profonds du céphalothorax, par le rostre antérieur garni des deux côtés seulement de très-petites dentelures, et surtout par le prolongement antérieur des joues ordinairement caréné et au moins de la moitié de la longueur du rostre.

l. oncopareia bredai nov. spec. 1853.

Pl. X, fig. 5, 6, 6, 7, 7 , et 8 .

Le plus parfait céphalothorax de cette espèce que j'aie vu, est représenté par la figure 5, dans laquelle le rostre a été ajouté hypothétiquement ${ }^{1}$ ) par des lignes ponctuées. Ce dernier offre sur chacun de ses angles latéraux deux dents assez longues, et une seule dent près de l'extrémité antérieure de son arête inférieure. La longueur totale de ce céphalothorax est, dans la ligne dorsale médiane, de 72 millimètres, dont 47 pour la partie principale antérieure, et 25 pour la postérieure. Sa hauteur est de 35 millimètres et sa largeur parait moins grande que

1) D'aprìs l'empreinte qui est. représentée sur la planche $\mathrm{X}$, fig. 8 . 
la hauteur. $\left.{ }^{1}\right)$ La hauteur reste à peu près la même dans toute la longueur. La ligne dorsale médiane, ainsi que le bord inférieur ne sont que très-faiblement arqués. Le bord postérieur est marginé d'un limbe qui est considérablement élargi, et quii est anguleux au milieu dans les deux tiers inférieurs de la hauteur; la surface de ce limbe est creusée de nombreux points très-petits. Immédiatement au dessủs de l'angle inféro-antérieur, le céphalothorax présente un lobe saillant très-court. Les deux côtés latéraux du céphalothorax sont fortement bombés et toute la surface est couverte de très-petites proéminences, plus nombreuses vers les bords inférieurs; ces proéminences deviennent plus: rares, ou même disparaissent dans les sillons et parfois aussi sur une grande étendue de la partie principale postérieure.

D'après l'échantillon figuré sur la planche $\mathrm{X}$, figure 6 , la surface des cinq premiers segments, et d'après celui qui est representé sur la même planche fig. 6', la surface des six premier's segments de l'abdomen est couverte de nombreux points creux. Les prolongements latéraux lamelleux sont également creusés de points; mais ici ces points sont beaucoup plus rapprochés les uns des autres et inégaux; ils sont remplacés sur la partie concave médiane de ces prolongements par de nombreuses lignes courtes, partant de points creux qui semblent avoir servi de points d'insertion à des poils fins.

Le premier segment de l'abdomen présente latéralement des lobes arrondis et très-courts. Les prolongements latéraux du second segment sont beancoup plus larges et sont terminés en une pointe bien moins aiguë, que ceux des trois segments suivants; le pénultième segment n'offre que des prolongements latéraux très-courts et obtusement anguleux ( $\mathrm{pl}, \mathrm{X}$, fig. $\sigma^{\prime}$ ) et en arrière de ceux-ci des excavations latérales assez larges.

Liabdomen avec la nageoire caudale est plus long que le céphalothorax; la longueur de ce demier est à celle du premier à peu près comme $6: 7$. D'après l'échantillon de la collection du Dr. VAN BREDA, figuré pl. $\mathrm{X}$, fig. 6', l'abdomen ne diminue que faiblement et assez régulièrement en largueur d'avant en arrière, et d'après le même échantillon, l'article basilaire des appendices latéraux de la nageoire caudale a une forme obliquement tétragonale, et sa surface tuberculeuse est creusée d'un sillon transversal étroit, mais assez profond. Le premier article de la branche -externe des appendices latéraux est assez grand; il offre le long de son bord externe une partie proéminente assez large, et à sa base, à côté de son large rebord proéminent, un renflement tuberculiforme; le dermier article de cette branche manque à l'échantillon figuré de la collection du Dr. VAN BREDa; mais dans un échantillon de la collection Géologique

1) La largeur ne peut être indiquée arec précision, parceque tous les échantillons qui ont être trouvés, sont plus ou moins comprimés et déformés dans la roche qui les renferme. 
de Harlem, dans lequel il a été conservé, cet article est d'une forme sémilunaire. Les lames mitoyennes de la nageoire caudale sont trés-larges, de forme trigonale, et leur surface tuberculeuse présente vers le côté interne un sillon assez large et assez profond, mais n'atteignant par les deux extrémités de la lame; la surface de cette dernière, ainsi que celle du premier article de la branche externe des appendices latéraux, est couverte de très-petites proéminences, qui offrent à leur côté postérieur des indices de l'insertion de poils fins.

Dans l'échantillon susmentionné du Musée Géologique de Harlem, le septième anneau de l'abdomen ou pièce médiane de la nageoire caudale a été conservée. Cette pièce est à peu près de la longueur des appendices latéraux; elle est assez large, inarticulée, et obtusement arrondie i son extrémité postérieure. Sa surface est creusée d'une multitude de points semblables à ceux des autres segments de l'abdomen; elle offre en oûtre deux sillons latéraux assez larges et assez profonds, qui partent de l'extrémité antérieure et qui n'atteignent pas la moitié de la longueur totale de la pièce.

La portion palmaire de la main droite ( $\mathrm{pl} . \mathrm{X}$, fig. 7) est aplatie en dehors et assez fortement convexe à son côté interne; elle est couverte de nombreuses proéminences, comme dentelées à leur bord antérieur et disposées par séries transversales assez irrégulières. J'ai vu des empreintes des doigts, qui montrent que ces derniers sont longs, droits et garnis sur leurs bords latéraux d'une série de tubercules dentiformes, pareils à ceux qui hérissent le bord interne de la portion palmaire de la main.

Je n’ai.vu jusqu'à présent que trois échantillons incomplets de la mąin gauche: celle-ci est beaucoup moins forte, elle est moins large et est couverte de tubercules beaucoup plus petits que la main droite; quelquefois ces tubercules sont remplacés par des points creux. La portion palmaire offre, dans deux des échantillons que j'ai vus, sur le bord externe une série de 5-6 tubercules proéminents et dans l'autre ( $\mathrm{pl} . \mathrm{X}$, fig. $\gamma^{\prime} a, b$ ) cette série tuberculeuse manque; l'un des trois échantillons offre sur son bord interne deux séries de tubercules spiniformes, tandis que dans les deux autres ce même bord n'offre qu'une seule rangée, formée de 3-4 de ces tubercules. Le côté interne de cette main est à peu près lisse; il est armé ( $\mathrm{pl} . \mathrm{X}$, fig. $\left.\gamma^{\prime} \not\right)$, près de son extrémité antérieure, d'un 'seul tubercule spiniforme, incliné en avant et situé sur la ligne longitudinale médiane. Les doigts de cette main sont grêles et très-longs. D'après un échantillon de ma collection, ils sont armés sur leur bord interne d'une rangée de gros tubercules dentiformes; et dans un échantillon de la collection du Dr. VAN BreDA, figuré pl. $\mathbf{X}$, fig. $6^{\prime} a, b$, ces tubercules sont petits et plus nombreux.

Un seul d'entre les échantillons que j'ai vus, et qui appartient à la collection du Musée Géologique de Harlem, montre deux articles d'une patte d'une des quatre paires postérieures; 
le second article de cette patte ambulatoire a environ 18 millimètres de longueur sur une largeur de 3 millimètres, tandis que le troisième article n'a que $7^{1 \frac{1}{2}}$ millimètres de longueur, sur une largeur à peu près égale à celle du second article. Iues deux articles sont lisses et assez fortement déprimés.

Les supports des yeux, (fig. 8) sont très-grands et de forme sémi-lunaire?

Je me fais un plaisir de dédier cette belle espèce au Président de la commission générale pour la carte et la description géologiques des Pays-Bas. Ce géologue a bien voulu mettre à ma disposition quelques-uns des exemplaires qui ont servi à la description que je viens de donner.

Gisement et Localités. - Cet interessant crustacé semble ne pas être rare dans le terrain Maestrichtien de Kunrnede. On en rencontre fréquemment des parties séparées, et bien que le test est assez souvent conservé, tous les échantillons que je connais sont plus ou moins mutilés. Mr. le Dr. De Ber, Paléontologiste zèlé, a eu l'obligeance de me prêter un moule intérieur et extérieur de cette même espèce. Ce moule a été trouvé dans le silex (hornstein) du bois d'Aix.

2. ONGOPAREIA? Heterodon nov. spec. 1853.

Pl. X, fig. $9 a, b$.

Je rapporte cette espèce avec doute au genre Oncopareia. Je n'en ai trouvé que les doigts, qui sont longs et très-forts. Le doigt immobile est déprimé au milieu; il est arqué et garni sur ses deux bords de plusieurs gros tubercules inégaux. Le doigt mobile scmble beaucoup moins courbé; il est pourvu sur son bord interne de grosses proéminences inégales et rapprochées. Le bord externe de ce doigt manque à tous les échantillons que j'ai vus. Toute la surface des doigts est lisse; on remarque seulement près de la ligne longitudinale médiane du doigt immobile quelques points creux.

observations. - Les deux doigts que je viens de mentionner sont réellement des parties de crustacé bien incompletes; aussi ce n'est qu' après de longues hésitations que je me suis enfin décidé à les figurer. - Comme ces pinces se rencontrent assez fréquemment, et comme celles qui ont été representées sont les plus parfaites que j’aie vus jusqu’ici, malgré plusieurs années de recherches, j'ai cru utile de les faire connaître, afin de les signaler à l'attention des Naturalistes.

Gisement et Localités. - Elle se rencontre dans les assises à Fissurirostra pectiniformis du Système Maestrichtien ì St. Pierre, Duché de Limbourg et à Grand-Lanaye en Belgique. 


\section{THALASSINIDAE.}

Gen. I. mesostyuds $H$. G. Bronn et Ferd. Roemer, 1852.

Paguri spec. Desmarest, Von Schlotheim, De France.

Callianassae spec. Mrune Edwardo, Fr. Ad. Roemer et Geinitz.

Caractères Génériques. - Céphalothorax dépourvu de rostre et ayant beaucoup de rapport avec celui des Callianassa, mais bien plus déprimé, fortement rétréci en arrière et dépourvu (d'après la figure qui en a été domnée par Mr. Geinitz) de sillon transversal. L'abdomen présente également des différences assez importantes. Le premier segment est de beaucoup le plus petit, le second n'est qu'un peu plus large et est le plus long (tandis que dans le genre Callianassa, il est plus large et seulement de la longueur du pénultième et même parfois plus court que celui-ci); ces deux segments réunis constituent un commencement de l'abdomen; en forme de coin; les quatre segments suivants sont très-grands, plus larges que longs et présentent des appendices latéraux en forme de nageoires, dont la destination est uu peu problématique; à la nageoire caudale, l'article moyen (le dernier segment du corps) parait quadrangulaire et présente en dessus un rélief de forme lancéolée. Les deux paires de nageoires latérales (qui sont aussi grandes que chez les Callianassa), ne sont pas plus grandes ici que l'article médian, et leur surface est creusée de quelques sillons rayonnants.

La différence de grandeur des deux pattes de la première paire des Mesostylus ne parait pas être bien notable, car on rencontre fréquemment des pattes gauches presque aussi grandes que les droites. Dans le genre Callianassa la patte antérieure gauche est constamment très-grèle et beaucoup moins forte que l'autre. Les pattes de la seconde paire dans les Callianassa n'atteignent par même le tiers de la largeur de la grosse serre droite, tandis que dans les Mesostylus, leur largeur égale et le plus souvent même dépasse les deux tiers de la largeur des grosses pattes antérieures.

Pendant un grand nombre d'années on n'a connu des Mesostylus que les pattes des deux premières paires antérieures, qui se rencontrent assez fréquemment dans divers étages du terrain crétacé supérieur, et qui avaient d'abord été rapportées au genre Pagurus par Desmarest et ensuite au genre Callianassa par Mr. Milne Edwards.

En 1849, Mr. Geinitz fit comnaître la plupart des autres parties de l'enveloppe tégumentaire (d'une espèce) de ces intéressants crustacés, et continua, à l'exemple du dernier des deux Naturalistes Français, à les ranger dans le genre Callianassa.

En 1852, les deux auteurs de la nouvelle édition de la Lethaea geognostica, ayant remarqué les différences importantes qui existent entre les parties trouvées dans le Grunsand 
de Kieslingswalda et figurées par Geivitz, et les parties correspondantes de la Callianassa subterranea actuellement vivante, créèrent pour les deux espèces fossiles (qu'ils réunissent, je pense à tort, en une seule) le genre Mesostylus qui ne tardera par d'être généralement adopté.

\section{Mesostylus FAUJASII}

Pl. $\mathrm{X}$, fig. $10 a, b$.

Bernard l'Hermite Faujas, 1798. Histoire Naturelle de la montagne de St. Pierre. pag. 179, tab. XXXII, fig. 5, 6 .

Traduction Hollandaise de Faujas par Pasteur, 1802. Natuntijie Historie van den St. Pietersberg. Blz. 241, 242, pl. XXXII, fig. 5, 6.

Pagurus Bernhardus Kruger, 1823. Unveltliche Naturgeschichte. tom. II, pag. 129.

Faujasii Desmarest, 1822. Hist. Nat. des Crustacés fossiles. pag. 127, pl. XI, fig. 2.

Mantell, 1822. The fossits of the south Downs, or illustrations of the Geology of Sussex. tab. XXIX, fig. 3.

von Schlotieim, 1823. Die Petrefaktenkunde auf ilirem jetzigen standpunkte. pag. 55.

DE France, 1825. Dictionnaire des Sciences Naturelles. tom. XXXVII, fig. 232.

Brons, 1838. Lethaea geognostica. pag. 736, tab. XXVII, fig. 23'.

Callianassa Faujasii Milne Edwands, 1837 Histoire Naturelle des Crustacés (dans Suites à Bufron). tom. II, pag. 310.

Minde Edwards in Lamarck, 1839. Histoire Naturelle des animanx sans vertètres. tom. V, pag. 287.

" "Fr. Ad. Roemer, 1840. Versteinerungen des Nord-dentschen Kreidegebirges. pag. 106.

Bronn, 1848. Index Palaeontologicus. - Oder Uebersicht der bis jetzt bekanntnen fossilen Organismen. Erste Abtheilung, pag. 203.

GEINITZ, 1849. Das Quadersandstein-gebirge oder Kreide-getirge in Deutschland. pag. 96.

Mesostylus Faujasii (pro parte) H. G. Rowuen, 1852. Lethaea geognostica, oder Abbitdung und Beschreibung der fur die Gebirgs-formationen bezeichnendsten Versteinerungen. $4^{\text {te }}$ Lieferung, pag. 354, tab. XXVII, fig. 23.

Les seules parties connues du corps de ce Mesostylus sont les pattes antérieures et unc partie des pattes de la seconde paire. 
Le premier article des pattes de la première paire'est court; sa largeur est à sa longueur comme 1: $1 \frac{1}{2}$; le second est assez fortement déprimé; sa largeur est à sa longueur comme 1:2; sa surface est lisse et son bord interne dentelé; la largeur du $3^{\text {me }}$ article est à sa longueur comme 3:5, il est subanguleux au milieu et sa section transversale est subpentagonale; comme l'article précédent il est seulement dentelé à son bord interne; sa partie médiane obtusement anguleuse, est couverte d'un grand nombre de tubercules assez gros, inégaux et très-rapprochés. Parmi ceux-ci il y en a quelques-uns sur la partie antérieure de l'article, qui s'élèvent sous forme de crête; immédiatement en avant de cette crête, on remarque une petite partie, arrondiesubtrigone, mobile et dont la surface est tout-à-fait lisse. La largeur du quatrième article ou carpe, est à sa longueur comme 3:4; il est irrégulièrement tétragonal, et offre en arrière une entaille assez profonde, pour la reception de la petite partie lisse que je viens de mentionner. Cet article est convexe-déprimé, ses angles latéraux sont aigus et finement serrulés, sa partie voûtée offre antérieurement une arête marginale aiguë, en avant de laquelle on remarque une partie comprimée; le bord antérieur de cette dernière, ainsi que l'arête marginale, sont surmontés d'une seule rangée de tubercules. La portion palmaire de la main est plus longue que large; elle est convexe-déprimée, fortement anguleuse, serrulée sur ses bords latéraux et tuberculeuse sur son bord antérieur; le doigt immobile offre constamment, au côté interne, une seule grosse dent obtuse, tandis que le bord correspondant du doigt mobile ou pouce en présente toujours deux; ces deux doigts sont très-gros et assez fortement courbés au sommet; leur longueur est, dans la plupart des individus, un peu moindre que la moitié de celle de la portion palmaire; dans quelques-uns de mes échantillons cependant, elle atteint presque les deux tiers de cette dernière dimension. La surface de la portion palmaire, ainsi que la partie antérieure du carpe, sont couvertes de nombreux petits tubercules. Sur les doigts, ainsi que vers le côté interne de la portion palmaire de la main, on remarque deux séries longitudinales de points enfoncés de forme sémilunaire. Le côté concave de ces enfoncements est tourné vers l'extrémité antérieure.

Les pattes de la seconde paire paraissent être toujours plus courtes que celles de la première. Je n'en connais que la main didactyle et le carpe. Ces deux articles sont déprimés et subtétragones. La main offre à peu près les mêmes ornements que l'article correspondant des pattes antérieures. Ses bords latéraux cependant ne sont point serrulés. Toute la surface du carpe est dépourvue de tubercules. Le bord interne de cet article est crenelé; sa plus grande largeur esî presque égale à sa plus grande longueur. Les doigts sont grêles, trés-faiblement courbés, anguleux et garnis sur chacun des angles d'une rangée de petits tubercules; ils sont constamment plus longs que la portion palmaire de la main. La largeur de cette dernière est à sa longueur comme $7: 8$. 
Rapports et Différences. - Le Mesostylus antiquus, dont j’ai reçu du Dr. Gernirz un échantillon provenant du Grünsand de Kieslingswalda, et que Mrs. Brons et Roemer réunissent, à titre de variété, au Mesostylus Faujasii, diffère de ce dernier par les pattes des deux premières paires plus petites, plus grêles, à main et à carpe tout-à-fait lisses et sans dentelures marginales; à pinces moins fortes et sans dents au côté interne; par le second article de la première paire creusé de deux sillons, et par le troisième article garni de deux séries granuleuses bien distinctes.

La patte de crustacé, provenant du plänersandstein de Leitmeritz, qui a été décrite par le Dr. Reuss ${ }^{1}$ ) sous le nom de Callianassa Faujasii, n'est pas le Pagurus Faujasii de Desirarest; elle me semble ne pas même dépendre du genre Mesostylus.

Gisement et Localités. - Les serres de cette belle espèce se rencontrent en assez grande abondance dans le Système Maestrichtien à Nedercanne, à St. Pierre, à Bemelen, à Geulhem, à Fauquemont et à Ransdal. Mr. VAN Riensdxk et Ladrent en ont trouvé à Wonck en Belgique. D'après le Dr. DE BEY on rencontre cette espèce dans la Lusberger-Breccie (Système Sénonien? Donont) du Louisberg près d'Aix-la-Chapelle. Suivant Mr. Fr. Ad. Roever, elle se trouve en Allemagne dans la craie supérieure près Gehrden, Quedlinbourg, Altenrode, Veckenstedt et Dulmen? Selon Manteli, elle existe dans la craie blanche de Lewes dans le Sussex, en Angleterre. Enfin, Mr. le sénateur Hermans Roemer de Hildesheim, vient de me communiquer des pattes antérieurs (exactement semblables à celles des environs de Maestricht) provenant des couches crétacées supérieures de la Nouvelle-Jersey, aux Etats-Unis d'Amérique.

\section{INCER'TAE FAMILIAE.}

Gen. I. AuLAcopoda nov. gen. 1853.

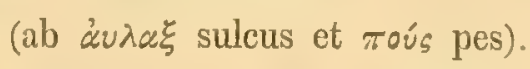

J'ai cru devoir proposer ce nouveau genre, pour y ranger la patte qui a été représentée sur la pl. X, fig. 11, parceque je n'ai pu la rapporter à aucun des genres connus.

1. AUlACopodia RLEMSdyKI nov. spec. 1853.

Pl. X, fig. 11 .

La portion palmaire de la main présente une forme trapézoïdale; elle est $1 \frac{112}{2}$ fois aussi longue à son côté interne, qu'à son côté externe; les pinces sont droites, friblement bourbées

1) Keuss, 1845. Versteinerungen der Böhmischen Kreide-formation. pl. V, fig. ว2. 
à l'extrémité et d'une longueur presque égale à celle de la portion palmaire. La pince immobile est quadridentée et la pince mobile quinquedentée au côté interne; la surface des deux pinces est creusée d'une série de points, placés au fond d'un sillon étroit et peu profond. La portion palmaire est creusée également vers le tiers externe de sa largeur, d'un sillon longitudinal étroit et profond, et n'atteignant pas l'extrémité antérieure. Immédiatement en avant de ce sillon on remarque un petit groupe de points creux. Sur la partie restante de la portion palmaire de la main, on observe encore deux séries longitudinales irrégulières de points peu profonds. Le quatrième article a une forme ovoïdale-oblique; il est assez fortement renflé et creusé de deux sillons, dont l'un au milieu et l'autre vers le quart externe de la largeur; cet article offre, vers sa moitié interne, une seule rangée un peu sinueuse de points creux.

Dimensions. - La main avec le carpe a une longueur de 27 millimètres; la plus grande largeur de la portion palmaire de la main est de 7 millimètres.

Gisement et Localités. - Cette espèce n’a été trouvée jusqu’à présent que dans la couche à Bryozoaires du Système Maestrichtien à St. Pierre. Elle semble être très-rare, je n’en ai vu jusqu’ì présent qu’un seul échantillon.

\section{(3. BRACHYURA.}

Gen. I. STEPHATOWETPPON nov. gen. 1853.

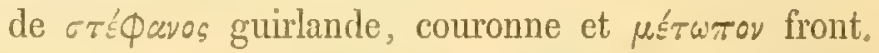

Caractères Géneriques. - Carapace déprimée, à face supérieure presque horizontale au milieu et inclinée aux deux extrémités; à peu près aussi large que longue, subpentagonale; à front assez large sémicirculaire-subtrigone et dirigé verticalement en bas, de façon à former un angle- droit avec l'axe du corps. 'Toute la surface de la carapace est couverte de très-petits tubercules mamillaires et dans les interstices de points creux excessivement petits, qui auront probablement servi de points d'insertion à des poils très-fins. Les bords latero-antérieurs sont droits et dirigés très-obliquement en avant. Ces bords et le front sont marginés d'un limbe saillant assez large et couvert de nombreuses granulations rapprochées. Le bord postérieur qui présente au milieu un prolongement sub-sémicirculaire (analogue à celui du Myctiris longicarpus) est marginé d'un bourrelet saillant. A la surface de celui-ci on ne remarque qu'une seule rangée de tubercules un peu plus gros que ceux qui s'élèvent sur le reste de la surface. Les régions de la curapace sont peu marquées. On y distingue cependant une région stomacale trapézoïdale, profondement canaliculée dans la diagonale de ses deux tiers antérieurs; une région génitale allongée et très-petite; des régions hépatiques et branchiales assez grandes, mais non 
nettement détachées du reste de la surface; enfin, une région cardiaque ovale-subtrigone et assez large. A la surface de cette dernière surtout et sur les régions branchiales, on remarque entre les granulations perlées et à peu près de grosseur égale, un grand nombre d'autres qui sont beaucoup plus petites.

Des membres je ne connais jusqu’ici que la main. La portion palmaire de celle-ci n'est qu'un peu plus longue que large. Elle est renflée et assez grosse; sa face supérieure est couverte de tubercules inégaux et en général plus gros que ceux de la carapace. Les pinces sont courtes et pointues; elles sont garnies d'une seule dent au côté interne. La pince immobile est lisse; la mobile au contraire, qui est fortement inclinée et de forme trigone-oblique, offre au milieu un angle, qui sépare sa surface supérieure en deux parties, dont l'interne est lisse, tandis que l'externe est couverte de tubercules très-rapprochés et inégaux.

Par la disposition du front et par quelques autres caractères de la carapace ce genre semble se rapprocher des Ocypoda, des Uca et des Myctiris. - Dans les Micippe, la carapace a une forme tout-à-fait différente et est armée de fortes épines marginales, le rostre est d'aillieurs proportionnellement beaucoup plus grand, et est terminé par des dents.

1. STEPHANOMEtopon GRANulatum nov. spec, 1853.

Pl. X, fig. $12 a, b, c$.

Cette espèce étant jusqu’ici la scule connue du genre, sera je pense, bien facile à reconnaitre.

Dbservations. - Les deux carapaces de Brachyures, du terrain crétacé de la Bohème, figurées par le Prof. Reuss ${ }^{1}$ ) et décrites par cet auteur sous le nom de Dromilites pustulosus, n'appartiennent certainement par au même Genre que l'espèce de Faxoë, pour laquelle Mr. Mrine Edwands a établi le genre Dromilites. Je pense en oûtre que les deux carapaces, décrites par l'auteur de Bilin, appartiennent bien plutôt à deux genres différents, qu'à des variétés d'une seule et même espèce.

Gisement et Localirés. - Cette espèce semble être rare. Elle n'a été trouvée jusqu'ici que dans la couche à Bryozoaires du Systéme Maestrichtien de la montagne de St. Pierre. Mr. le Professeur De Koninck m'a communiqué une main de la même espèce, provenant du terrain Maestrichtien de Ciply près Mons.

1) Revss, 1845-1846. Versteinerungen der Böhmischen Kreide-formation. taf. VII, fig. 26 und 29. 


\section{TABLE APHABÉTIOUE DES ESPËCES.}

\begin{tabular}{|c|c|c|c|c|c|c|c|}
\hline Aulacopodia Riemsdyki................. & $\begin{array}{l}\text { Page. } \\
125\end{array}$ & \begin{tabular}{|c|} 
Planche. \\
$\mathbf{X}$
\end{tabular} & $\begin{array}{c}\text { Figure. } \\
11 .\end{array}$ & Cythere propinqua......... & $\begin{array}{r}\text { Page. } \\
78\end{array}$ & $\begin{array}{c}\text { Planche. } \\
\text { V }\end{array}$ & $\begin{array}{c}\text { Iigure. } \\
9 .\end{array}$ \\
\hline Bairdia arcuata . . . . . . . . . . . . . . & 59 & V & 3. & " pulchella..................... & 76 & IX & 1. \\
\hline "Var. B. Gracilis.......... & 60 & V & 4. & " Var. B.................... & 76 & $\mathbf{I X}$ & 2. \\
\hline "Var. G. Lunata............. & 60 & & & pancturata.............. & 81 & VI & 1. \\
\hline subdeltoidea.. & 56 & VIII & 4. & quadridenta............... & 90 & VI & 12. \\
\hline subgiobosa.... & 55 & VIII & 3. & radiosa............ & †9 & V & 10. \\
\hline Cyprella Koninckiana. . & 115 & IX & 12. & sagittata............... & 87 & VI & 8. \\
\hline 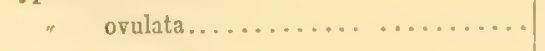 & 114 & IX & 11. & semicancellata............ & 102 & VII & 9. \\
\hline 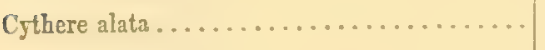 & 107 & IX & 10. & serrulata............... & 104 & IX & 9. \\
\hline " $\quad \operatorname{arenosa} \ldots \ldots \ldots \ldots \ldots \ldots \ldots$. & 91 & VII & 1. & strangulata......... & 84 & VI & 5. \\
\hline celleporacea................. & 101 & VII & 8. & striato-costata... & 77 & V & S. \\
\hline " cerebralis....... & 83 & VI & 3. & subtetragona....... & 80 & V & 11. \\
\hline complanata.... & 89 & VI & 10. & trigonoptera ................... & 109 & VII & 12. \\
\hline " concentrica...... & 71 & VIII & 8. & umbonella.................... & 85 & VI & 6. \\
\hline Var. $B \ldots \ldots \ldots \ldots \ldots$ & 71 & V1II & $8^{\prime}$ & 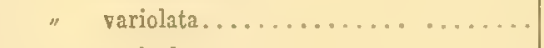 & 91 & VII & 2. \\
\hline$" \quad \operatorname{cristata} \ldots \ldots \ldots \ldots \ldots \ldots \ldots \ldots$ & 112 & $\mathbf{X}$ & 4. & vesiculosa .............. & 82 & V1 & 2. \\
\hline 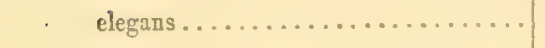 & 78 & IX & 3. & Cytherella auricularis....... . & 50 & IV & 19. \\
\hline " elegantula........ & 94 & VII & 4. & denticulata........ & 51 & V & 1. \\
\hline euglypha......... & 74 & V & 7. & Munsteri........ & 48 & VIII & 2. \\
\hline " eximin......... & 96 & VII & 6. & ovata................................. & 45 & VIII & 1. \\
\hline " Favrodiana.................. & 70 & VIII & 7. & Williamsoniana.............. & 52 & V & 2. \\
\hline 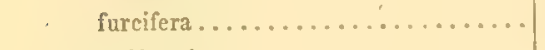 & 73 & VIII & 9. & Cytheridea Harrisiana . . . . . . . . . . . . & 63 & V & 5. \\
\hline 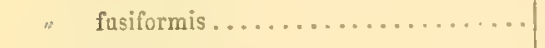 & 69 & VIII & 6. & Jonesiana ............... & 64 & VIII & 5. \\
\hline 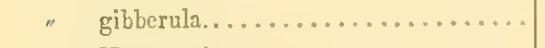 & 84 & VI & 4. & ovata........ & 63 & V & 6. \\
\hline Hagenowi..................... & 110 & $\mathbf{X}$ & 2. & Mesostylus Faujasii....... & 123 & $\mathrm{X}$ & 10. \\
\hline hieroulyphica...... & 92 & IX & 5. & Mitella Darwiniana......... & 10 & I & 8-16. \\
\hline , horridula... & 95 & VII & 5. & " glabra........... & 17 & II & 4-12. \\
\hline interrupta.... & 74 & VIII & 10. & valida...... & 14 & II & $1-3$ \\
\hline " Koninckiana.. & 100 & IX & 7. & Oncopareia Bredai ........... & 118 & $\mathbf{X}$ & $5 \cdot 8$ \\
\hline$" \quad l a b y$ & 93 & VII & 3. & P beterodon............ & 121 & $\mathbf{x}$ & 9. \\
\hline laticristata... & 108 & VII & 11. & Scalpellum Darwinianum.... & 36 & IV & 6-12. \\
\hline Iepida............ & 89 & VI & 11. & elongatam.... & 32 & III & $18-20$. \\
\hline longispina............ & 86 & VI & 7. & gracile & 26 & II & 18. \\
\hline macrophthalme.. & 86 & IX & 4. & & & III & 1.9. \\
\hline 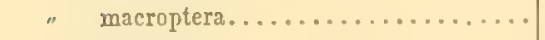 & 111 & $\mathbf{X}$ & 3. & Hagenowianum .. & 39 & IV & $13-16$ \\
\hline minuta....... & 109 & $\mathrm{X}$ & 1. & $\operatorname{maximum} . . .$. & 23 & II & 13-17. \\
\hline multilamella. & 80 & V & 12. & pulchellum. & 34 & IV & 1.5. \\
\hline orchidea.... & 88 & VI & 9. & & & III & 21,22 . \\
\hline 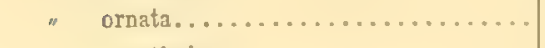 & 103 & IX & 8. & pygmaenm..... & 29 & III & 10.17 . \\
\hline ornatissima............. & 97 & IX & 6. & radiatum........ & 41 & IV & 17,18 . \\
\hline " Var. A. Nodulosa... & 98 & VII & 7. & Stepharometopon granulatura. & 127 & $x$ & 12. \\
\hline " phylloptera. & 106 & VII & 10. & Verruca prisca...... & 4 & I & $1-7 !$ \\
\hline
\end{tabular}






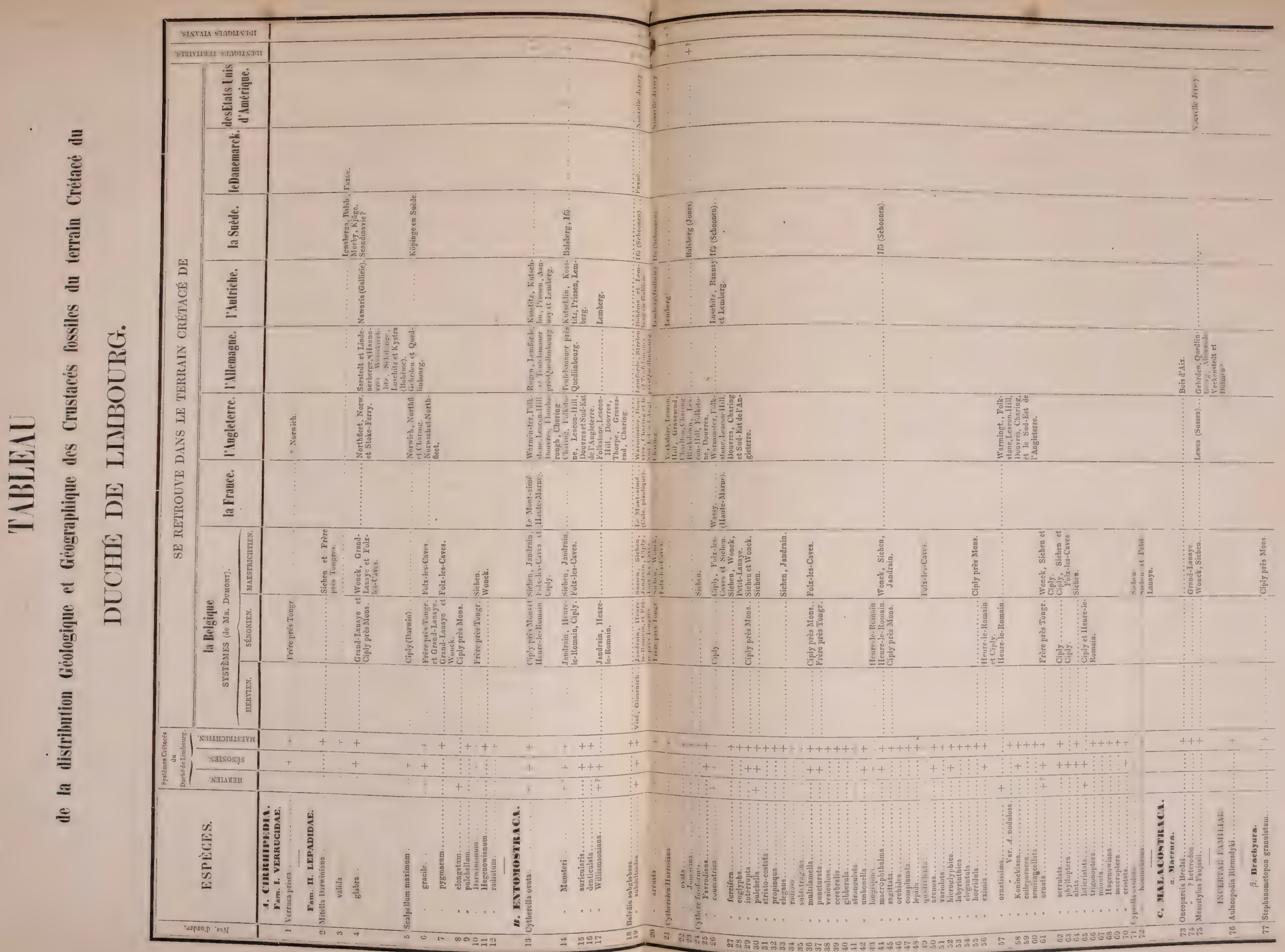



EXPLICATION DES PLANGHES. 


\section{PLANCHE I.}

Figures 1-7. Verruca prisca Darwin in litter. Page 4.

Fig. 1, a. Scutum fixe d'un individu attaché par le côté droit, vu à l'extérieur et grossi douze fois la grandeur naturelle. (Toutes les autres valves de la Verruca prisca figurées sur la même planche sont pareillement grossies douze fois la" grandeur naturelle).

b. Le même, vu du côté extérieur.

" 2,a. Scutum mobile d'un individu fixé par le côté gauche, grossi et vu du côté externe.

๖. Le même scutum, vu du côté interne.

3, a. 'Tergum mobile, sans doute du même individu, grossi et vu du côté externe.

Ђ. Le même tergum, viu du côté interne.

4. Tergum fixe, d'un individu attaché par le côté gauche, 1) grossi et vu du côté interne.

" 5,a. Tergum fixe, d'un individu fixé par le côté droit, vu à l'extérieur et grossi.

b. Le même tergum, vu du côté interne.

"6. Tergum fixe, d'un individu qui a été fixć par le côté droit, grossi et vu du côté carérial.

7. Carina ? 2) vue du côté externe et grossie.

- 7', a. Carina? vue dans une autre position, pour montrer la disposition des stries d'accroissement à la surface des aires articulaires.

b. La même valve, vue du côté interne:

Figures 8-16. Mitella Dartiniana Bose. Page 12.

Fig. 8, a. Scutum, vu ì l'extérieur et grossi au double de sn grandeur naturelle.

b. Le même, vu à l'intérieur et agrandi de même.

c. Trait, indiquant la grandeur naturelle.

. 9, a. Tergum, vu ì l'extérieur et grossi au double de sa grandeur naturelle.

b. Le même, grossi de même et vu du côté interne.

$c$. Trait, indiquant la grandeur naturclle.

" 10, a. Carina, vue à l'extérieur, restaurée par des lignes ponctuées et grossie au double de la grandeur naturelle.

Fig. 10, ъ. La même, vue latéralement et grossie de même.

c. La même restaurée, grossie et vue en dedans.

d. Trait, indiquant la grandeur naturelle.

e. Section transversale de la carina grossie, près de l'extrémité inférieure.

$f$. Section transversale vers le tiers supérieur de la hauteur.

" 11, a. lostrum, vu de son côté dorsal, et grossi au double de sa grandeur naturelle.

b. Le même, grossi de même et vu latéralement.

c. Le même, grossi de même et vu en dedans.

d. Trait, indiquant la grandeur naturelle.

$e$. Section transversale du rostrum, grossi au double, vers le quart inférieur dẻ sa hauteur.

f. Section transversale du même, vers la moitié de sa hauteur, au quadruple de la grandeur naturelle.

"12, a. Latus du verticille inférieur, grossi huit fois la grandeur naturelle et vu du côté externe.

ひ. Le même, grossi de même et vu du côté interne.

c. Trait, indiquant la grandeur naturelle.

13, a. Latus de la paire supérieure, grossi buit fois la grandeur naturelle et vu du côté extéricur.

b. Trait, indiquant la grandeur naturelle.

" 14, a. Autre latus supérieur, grossi de même et vu du même côté.

b. Le même, vu du côté interne.

" 15, a. Epine du pédoncule, grossie huit fois la grandeur naturelle et vue du côté esterne.

๖. La même, vue du côté interne.

c. La même, vue du côté latéral.

c. Trait indiquant la grandeur naturelle.

"16, a. Autre épine du pédoncule, grossie de même et vue du côté externe.

๖. La même, grossie de même et vue du côté interne.

1) Mr. DARWIN pense que très-probablement cette valve puisse provenir da même individa, dont proviennent les scutum et tergum mobiles.

2) J'ai déja fait observer dans la Ulescription de cette espèce, qu'il est impossible d’affirmer, si cette valve est une carine ou un rostrum, sans savoir si elle provient d'un individu fisé par le côté gauche ou d'un individu fizé par le côté droit. 


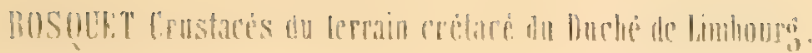
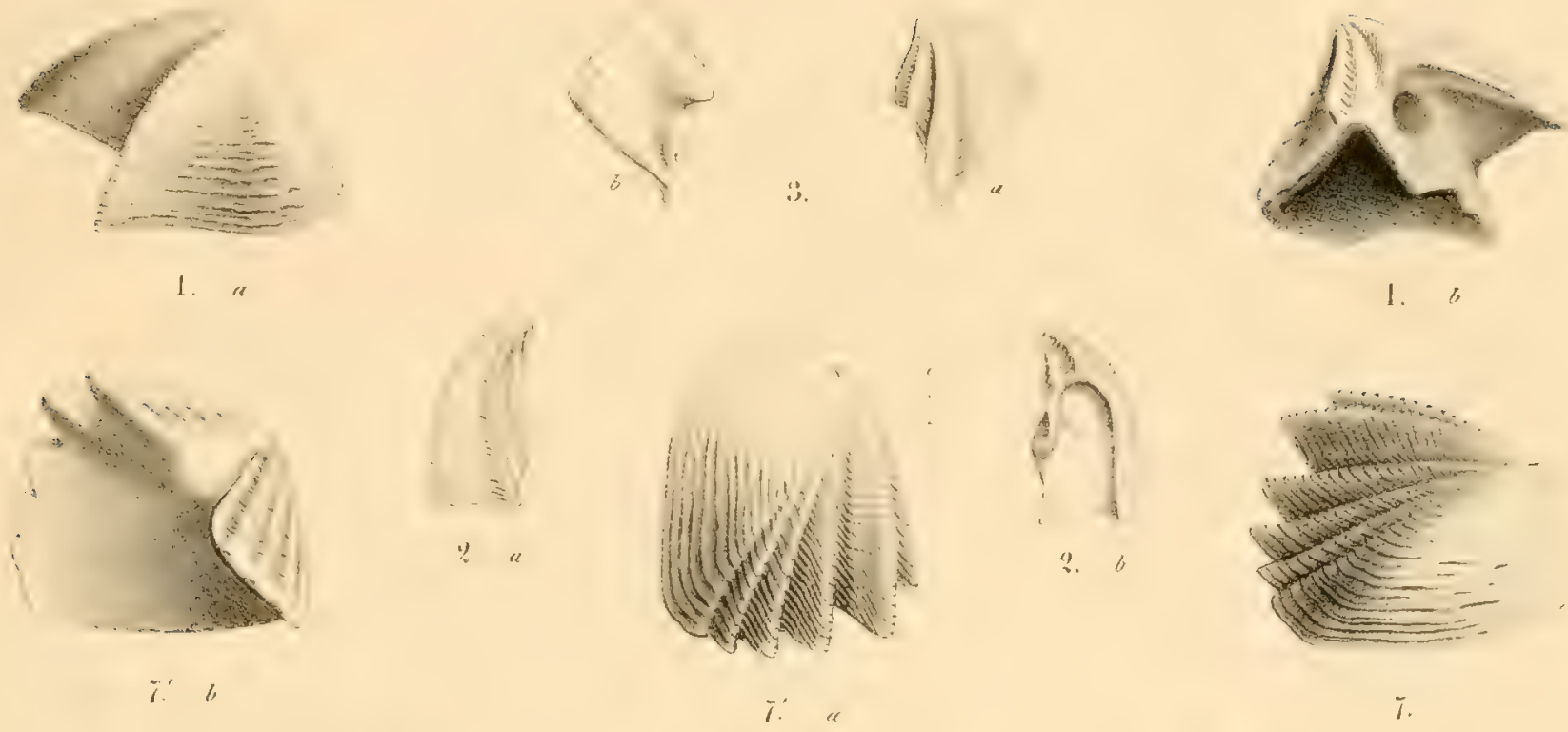

I."
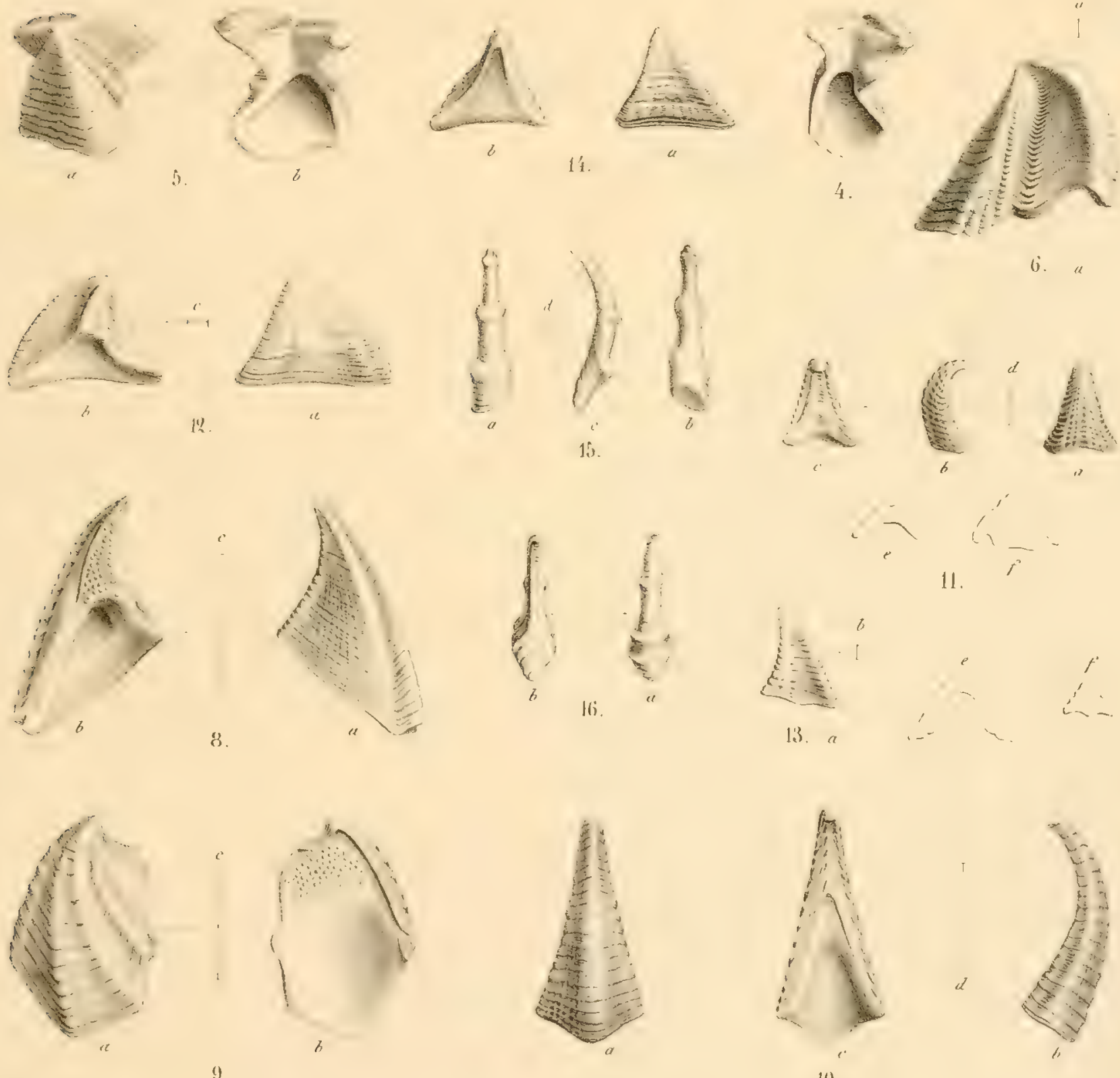

10. 




\section{PLANCHE II.}

Figure 1, 2, 3. Mitella valida Steenstr., spec. Page 14.

Fig. 1, a. Scutum, vu à l'extérieur et grossi au double de sa grandeur naturelle.

๖. Le même, vu à l'intérieur et grossi de même.

c. Trait, indiquant la grandeur naturelle.

2, a. Tergum, agrandi au double et vu du côté externe.

b. Trait, indiquant la grandeur naturelle.

3. a. Carina, vue du côté externe, de grandeur natur.

b. La même, vue latéralement.

c. Section transversale de la carina, près de l'extrémité inférieure.

d. Section transversale de la même, vers la moitié de la hauteur.

Figure 4-12. Mitella glabra Roemer, spec. Page 17.

Fig. 4, a. Scutum d'un jeune individu, vu à l'extérieur et grossi an double de la grandeur naturelle.

b. Trait, indiquant le grandeur naturelle.

5, a. Tergum d'un individu adulte, vu à l'extérieur et agrandi au double.

6. Le même, grossi de même et vu à l'intérieur.

c. Trait; indiquant la grandeur naturelle.

6, a. Carina, vue à l'extérieur, et grossie au double de la grandeur naturelle.

b. La même, vue du côté interne.

c. La même, vue latéralement.

1. Trait, indiquant la grandeur naturelle.",

c. Section transversale de la carina, près de l'extrémité inférieure.

7, $a$. Rostrum, grossi au double de sa grandeur naturelle et vu ì l'extérieur.

b. Le même, grossi de même et vu à l'intérieur.

c. Le même, grossi de même et vu latéralement.

d. Trait, indiquant la grandeur naturelle.

8. Fragment du latus supérieur, grossi au double de sa grandeur naturelle, et provenant sans doûte d'un individu adulte.

$9, a$. Latus de la paire supérieure, entier, agrandi au double et provenant probablement d'un individu sub-adulte.

b. Le même, grossi de même et vu du côté interne.

$10, a$. Latus du verticille inférieur, (probablement du côté du rostrum) vu à l'extérieur et grossi au quadruple de sa grandeur naturelle.

b. Le même latus, grossi de même et vu du côté interne.
Fig. 10, c. Traits, indiquant les dimensions naturelles.

" 11. a. Latus du même verticille (probablement du côté latéral médian) vu á l'extérieur et grossi au double de la grandeur naturelle.

b. Trait, indiquant la grandeur naturelle.

" 11 , $a$. Latus du même verticille (probablement de la même place à peu peu près) vu à l'extérieur et grossi au double de la grandeur naturelle.

b. Traits, indiquant les dimensions naturelles.

" 12, a. Latus du même verticille, (probablement du côté de la carina) vi à l'extérieur et grossi au double de sa grandeur naturelle.

b. Le même latus, vu du côté interne.

Figure 13-17. Scalpellum maximum J. Sowerby, spec. Page 23.

Fỉg. 13, a. Carina, de la collection du Dr. J. MüLLER, vue du côté externe et grossie au double de la grandeur naturelle.

๖. La même, vue du côté latéral.

$c$. Trinit, indiquant la grandeur naturelle.

" 14, a. Fragment d'une carina, également de la collect. du Dr. MüLLER, de la même var., agrandie au triple de la grandeur naturelle et vue du côté dorsal.

b. Le même fragment, grossi de même et vu latéralement.

c. Section transversale du fragment, à l'endroit de sa troncature accidentelle.

d. Trait, indiquant la grandeur du fragment.

" 15, a. Tergum d'une variété de cette espèce, vu à l'extérieur et grossi an double de sa grandeur naturelle.

b. Trait, indiquant la grandeur naturelle.

"16. Tergum incomplet d'un jeune individu, peutêtre de la variété Il de Mr. DARtwin, vu à l'extérieur et grossi au quadruple de'la grandeur naturelle.

"17, a. Latus rostral, vu à l'extérieur et grossi au quadruple de la grandeur naturelle.

๖. Trait, indiquant la grandeur naturelle.

Figure 18. Scalpellum gracile Bosquer, Page 26.

Fig. 18, a. Latus carénal, vu à l'extérieur et grossi au quadruple de la grandeur naturelle.

b. Le même, grossi de même et vu du coté interne.

c. Trait, indiquant Ja grandeur naturelle. 

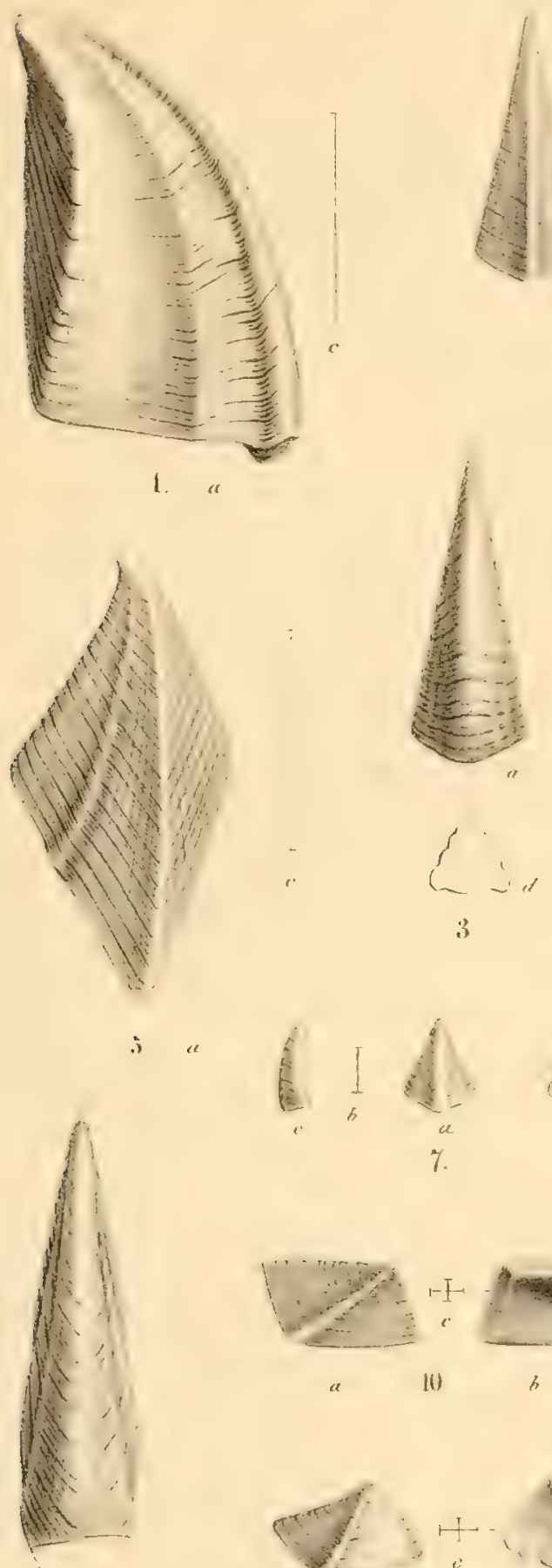

$1+4$
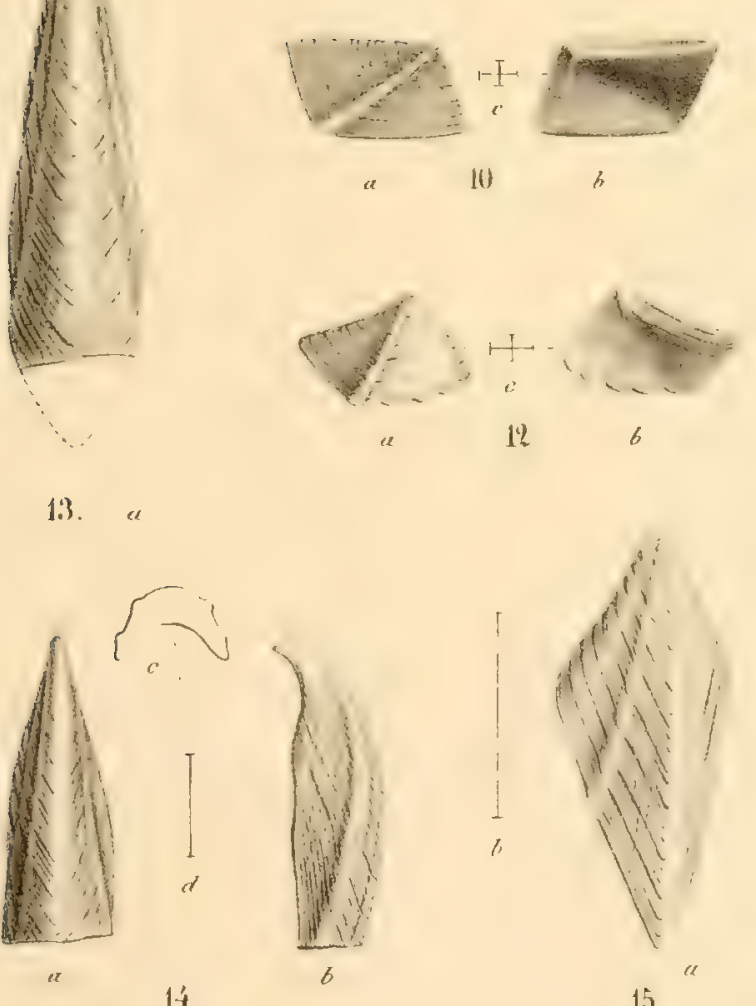

14 .
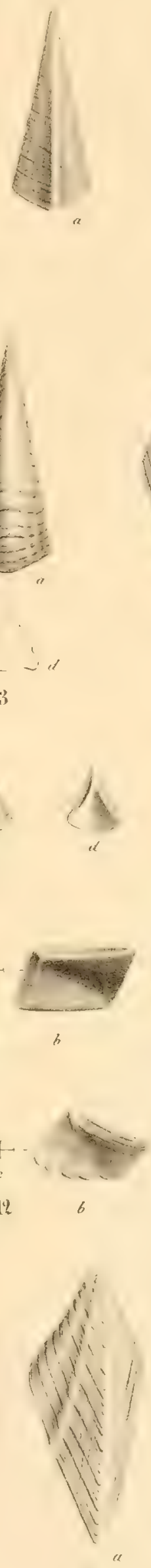

15.
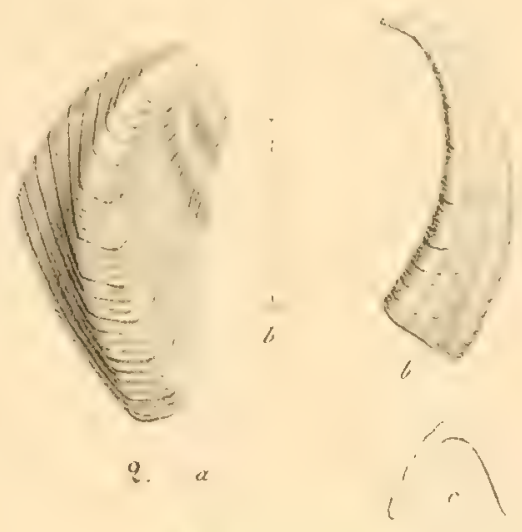

3.
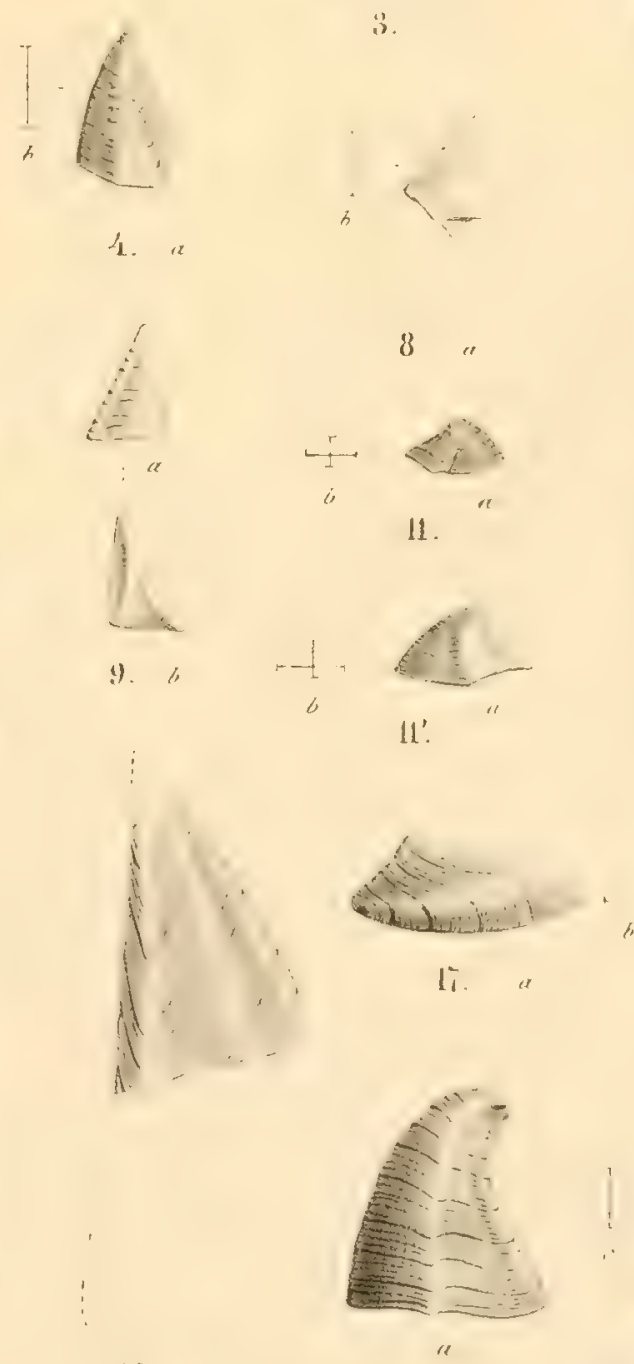

16.

13
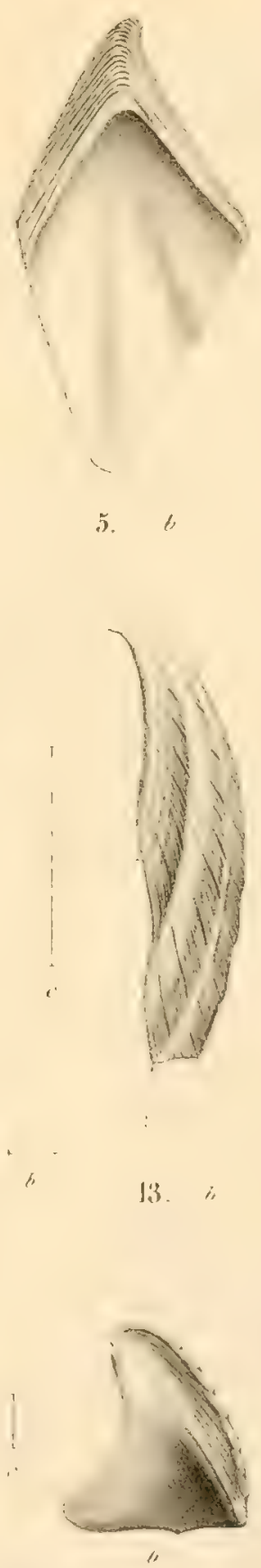




\section{PJANCHE III.}

Figure 1-9. Scalpellum gracile Bosquet. Page 26.

Fig. 1, a. Carina, rue du côté externe, et grossie au double de la grandeur naturelle.

๖. La même, vue latéralernent.

c. Trnit, indiquant la grandeur naturelle.

" 2, a. Carina d'un autre individu, vue à l'extérieur, de grandeur naturelle.

b. Là même, vue du côté latéral.

3. Tergum incomplet, vu à l'extérieur et grossi au double de la grandeur naturelle.

4. Latus supérieur, vu ì l'extérieur et grossi au quadruple de la grandeur naturelle.

5, a. Latusocarénal, vo à l'extérieur dans sa position naturelle (suivant MIr. DixoN) et grossi au quadruple de sa grandeur naturelle.

i. Le même latus, grossi de même et vu du même côté, dans sa position naturelle, d'après Mr. DARTIN.

c. Latus carénal d'un vieil individu, grossi au quadruple, vu du côté interne, dans sa position uaturelle d'aprés Mr. DrxoN.

7. Latus rostral, vu du côté externe et grossi huit fois la grandeur naturelle.

$\therefore$, a. Rostrum, vu du côté externe et grossi au quadruple de la grandeur naturelle.

b. Le même, grossi de même et vu du côté interne.

c. Le même, grossi de même et vu latéralement.

" ؛. a. Plaque du pédoncule, vue à l'extérieur et grossie huit fois la grandeur naturelle.

b. La même, grossie de même et rue du côté interne.

Fig. 10-17. Scalpellum pyğmaeum Bosquet. Page 29.

. 10, a. Carina, vue de son côté dorsal et grossie au donble de la grandeur naturelle.

b. La mêrne grossie de même et vue latéralement.

c. Section transversale vers le tiers supérieur de la hauteur.

7. Section transversale, près de l'extrémité inférieure.
Fig. 11, a. Scutum incomplet, vu.à l'extérieur et grossi au quadruple de la grandeur naturelle.

6. Le même, grossi de mểme et vu du côté interne.

"12, a. Tergum, vu du côté extérieur et grossi au double de la grandeur naturelle.

๖. Trait, indiquant la grandeur naturelle.

13. Latus supérieur vu du côté externe et grossi huit fois la grandeur naturelle.

14, a. Latus rostral, vu du côté externe et grossi huit fois la grandeur naturelle.

し. Le même, grossi de même et vu du côté interue.

" $15, a$. Rostrum, vu à l'extérieur, et grossi au quadruple de la grandeur naturelle.

l. Le même, grossi de même et vı ̀̀ l'intérieur.

c. Le même, grossi de même et vụ du côté latéral.

16, a. Ecaille du pédoncule, vue à l'extérieur et grossie huit fois la grandeur naturelle.

b. La même, grossie de même et rue du côté interne. " 17, a. Plaque d'un autre endroit du pédoncule, vue à l'extérieur et grossie huit fois la grandeur naturelle.

๖. La même plaque, grossie de même et vue du côté interne.

Figure 18-20. Scalpellum elongatum Bose. Page 32.

Fig. 18, a. Carina, rue à l'extérieur, de grandeur naturelle.

乙. La même, vue à l'intérieur.

c. La même, vue latéralement.

" 19, a. Carina incomplète, vue à l'extérieur ct grossie au double de la grandeur naturelle.

b. La même, grossie de même et rue latéralement. " $20, a$. Scutum incomplet, vu à l'extérieur.

6. Trait, indiquant la grandeur de l'échantillon.

Figure 21, 22. Scalpellum pulchellum Bosa. Page 34.

Fig. 21, a. Latus carénal, vu a l'extérieur et grossi huit fois la grandeur naturelle.

๖. Trait, indiquant la grandeur naturelle.

" 22, a. Latus rostral, vu du côté externe et grossi huit fois la grandeur naturelle.

๖. Trait, indiquant la grandeur naturelle. 

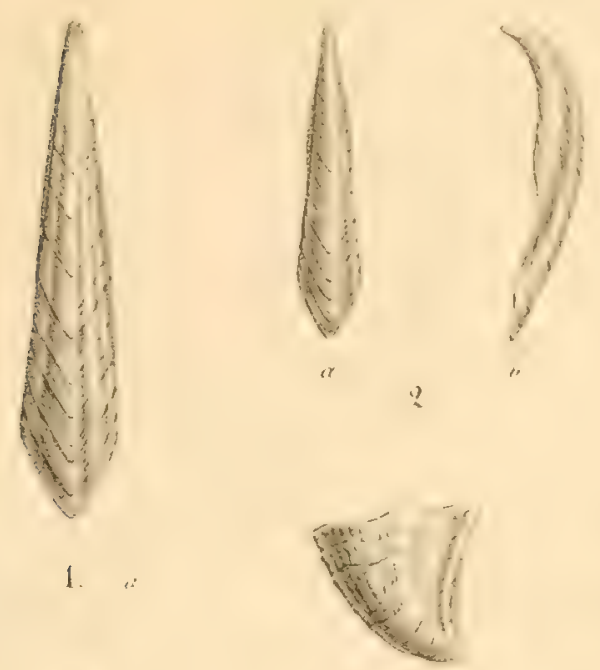

南
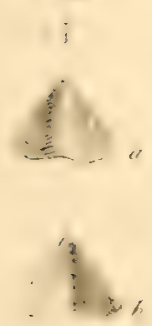

is
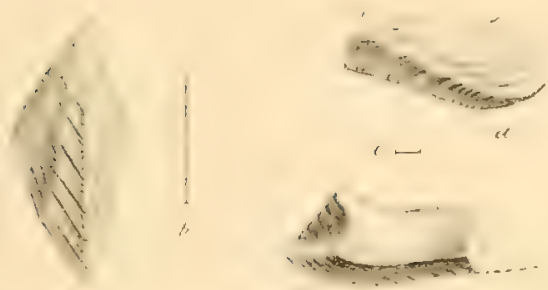

(4)

14.
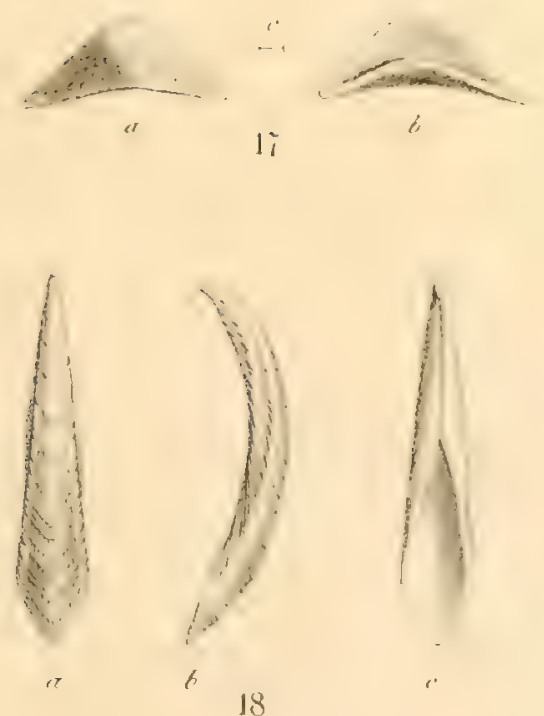

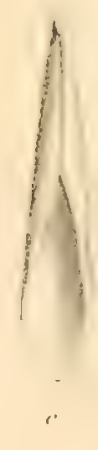

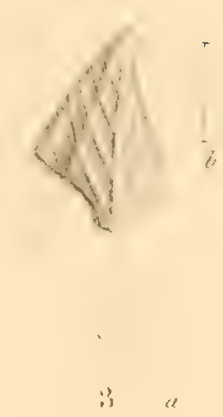
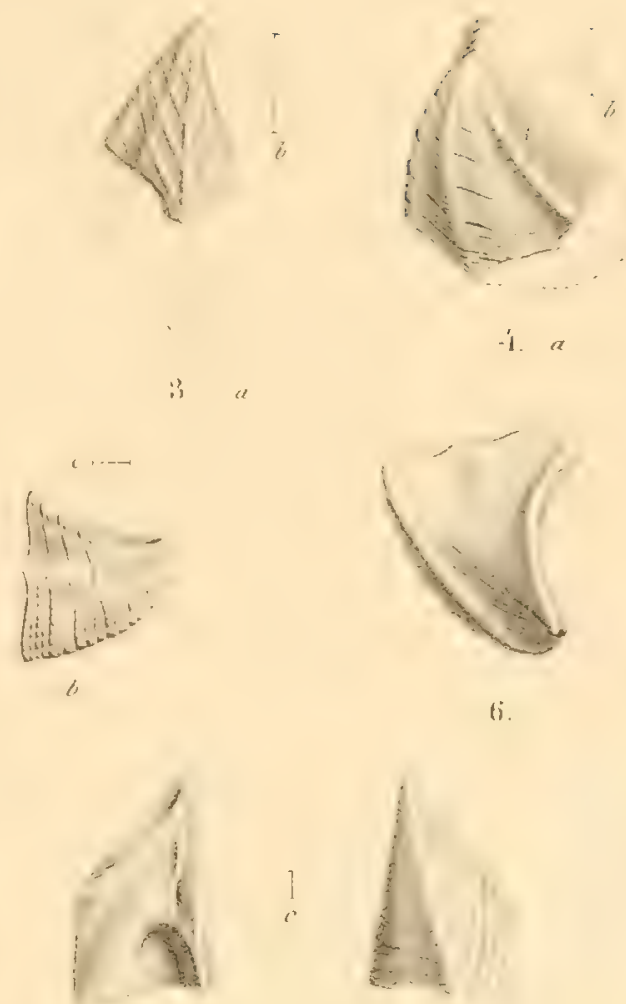

i.
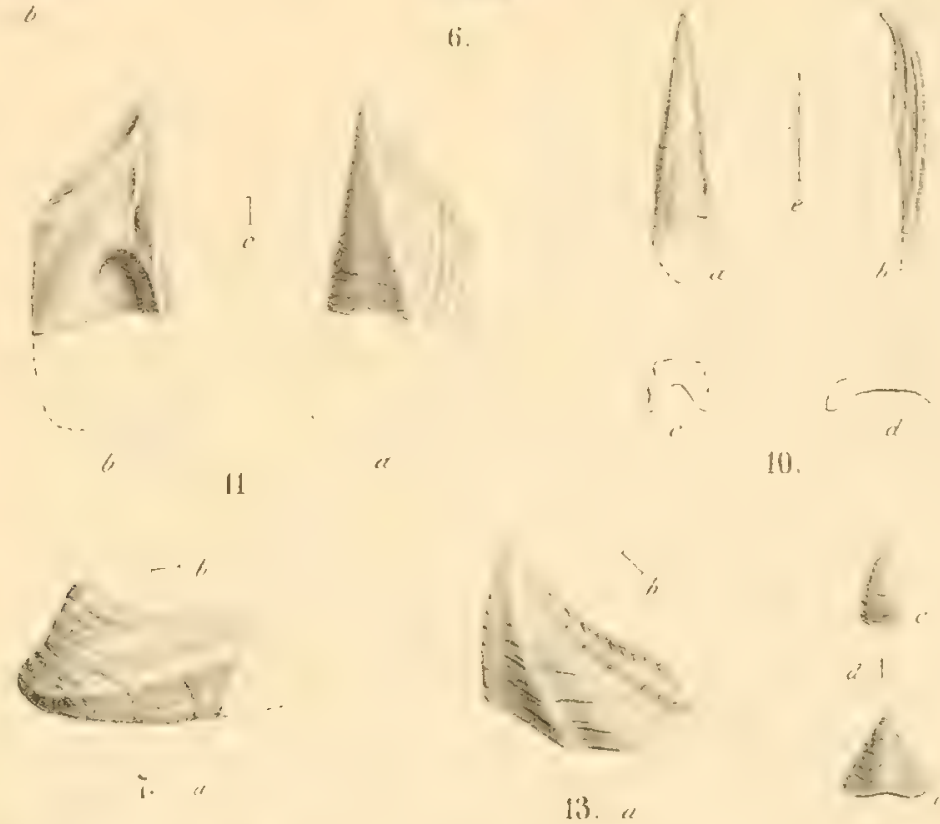

10.
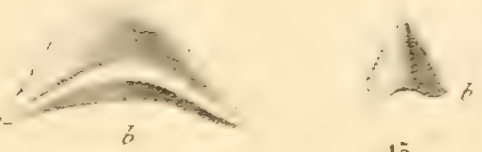

15

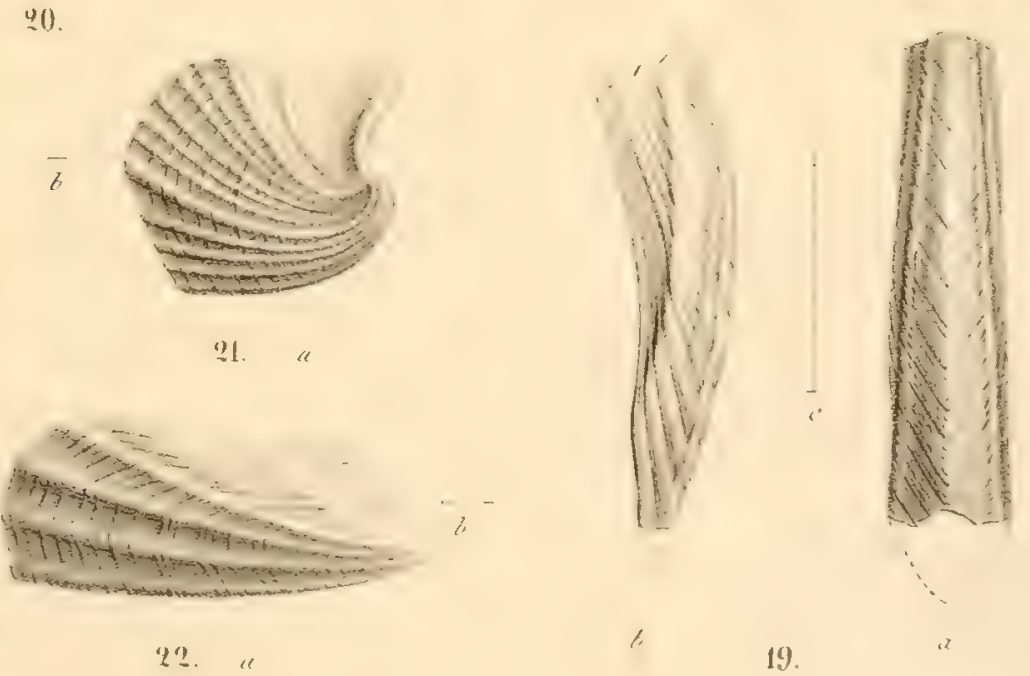






\section{PLANCHE IV.}

Figure 1-5. Scalpellum pulchellura BosQ. Page 34.

Fig. 1, a. Tergum, vu à l'extérieur et grossi au quadruple de la grandeur naturelle.

๖. Le même, grossi de même et vu du côté interne.

$c$. Trait, indiquant la grandeur naturelle.

. 2, a. Latus supérieur, vu du côté externe et grossi huit fois la grandeur naturelle.

b. Trait, indiquant la grandeur naturelle.

3, $a$. Latus carénal, d'un individu adulte, vu à l'extérieur et grossi huit fois la grandeur naturelle.

๖. Trait, indiquant la grandeur naturelle.

4, a. Latus carénal, provenant sans doute d'un vieil individu, vu à l'extérieur et grossi huit fois la grandeur naturelle.

5, a. Rostrum, vu du côté externe et grossi huit fois la grandeur naturelle.

b. Le même, grossi de même et $\mathbf{v u}$ en dedans.

$c$. Trait, indiquant la grandeur naturelle.

I'igure 6-12. Scalpellum Darwinianum Bosa. Page 36.

Fig. 6, $a$. Carina, vue de son côté dorsal (dans une position renversée) grossie au quadruple de la grandeur naturelle.

b. La même, grossie de même et vue latéralement.

c. Trait, indiquant la grandeur naturelle.

$7, a$. Scutum, vu à l'extérieur et grossi au quadruple de la grandeur naturelle.

b. Le même, grossi de même et vu du côté interne.

c. 'Trait, indiquant la grandeur naturelle.

8, $a$. Tergum, vu du côté externe et grossi au quadruple de la grandeur naturelle.

b. Le même, grossi de mềne et viı du côté interne.

c. Trait indiquant la grandeur naturelle.

9. Latus supérieur, nu du côté externe et grosaj huit fois la grandeur naturelle.

* 10, a. Latus rostral, vu du côté extérieur et grrossi huit fois la grandeur naturelle.

b. Trait, indiquant la grandeur naturelle.

"11. Latus carénal, vu du côté́ externe et grossi huit fois la grandeur naturelle.

" İ, a. liostrum, vu de son côté dorsal et grossi au quintuple de la grandeur naturclle.

" 12, b. Le même, grossi de même et vu du côté interne.

c. Le même, grossi de même et vu du côté latéral.
Fig. 12, $d$. Section transversale, vers le tiers supérieur de la hauteur.

e. Section transversale, vers le quart inférieur de la hauteur.

$f$. Trait, indiquant la grandeur naturelle de l'échantillon.

Figure 13-16. Scalpellum Hagenowianum Bosever. Page 39.

Fig. 13, a. Carina incomplète, vue du côté externe et grossie au quadruple de la grandeur naturelle.

6. La même, grossie de même et vue du côté latéral.

$c$. Trait, indiquant la grandeur naturelle.

d. Section transversale de la carina fortement grossie, à l'endroit de sa troncature accidentelle.

"14, a. Tergum, vu à l'extérieur et grossi iau quadruple de la grandeur naturelle.

b. Le même, grossi de même et vu du côté interne.

c. Trait, indiquant la grandeur naturelle.

15. Latus rostral, nı dı côté externe et grossi huit fois la grandeur naturelle.

$16, a$. Rostrum, vu du côté externe et grossi huit fois la grandeur naturelle.

Ђ. Le même, vu latéralement.

Figure 17, 18. Scalpellum radiatum Bose. Page 41.

Fig. 17, a. Scutum restauré par des lignes ponctuées, vu du côté externe et grossi au quadruple de la grandeur naturelle.

๖. Le même, grossi de même et vu du côté interne.

c. Trait, indiquant la grandeur naturelle.

" 18, a. Latus supérieur, vu du côté externe (figuré dans une mauvaise position) et grossi au quadruple de la grandeur naturelle.

6. Section transversale de ce latus, à côté du bord tergal.

c. Trait, indiquant la grandeur naturelle.

Figure 19. Cytherella auricularis Bosquet. Page 50.

Fig. 19, a. Valre gauche, provenant du Syst. Maestrichtien de Geulhem, vue en dessus. De ma collection.

6. Carapace entiére, provenant de la même localité, vue du côté supérieur. De ma collection.

c. La même, vue du côté inférieur.

d. La même, vue par l'extrémité antérieure. 

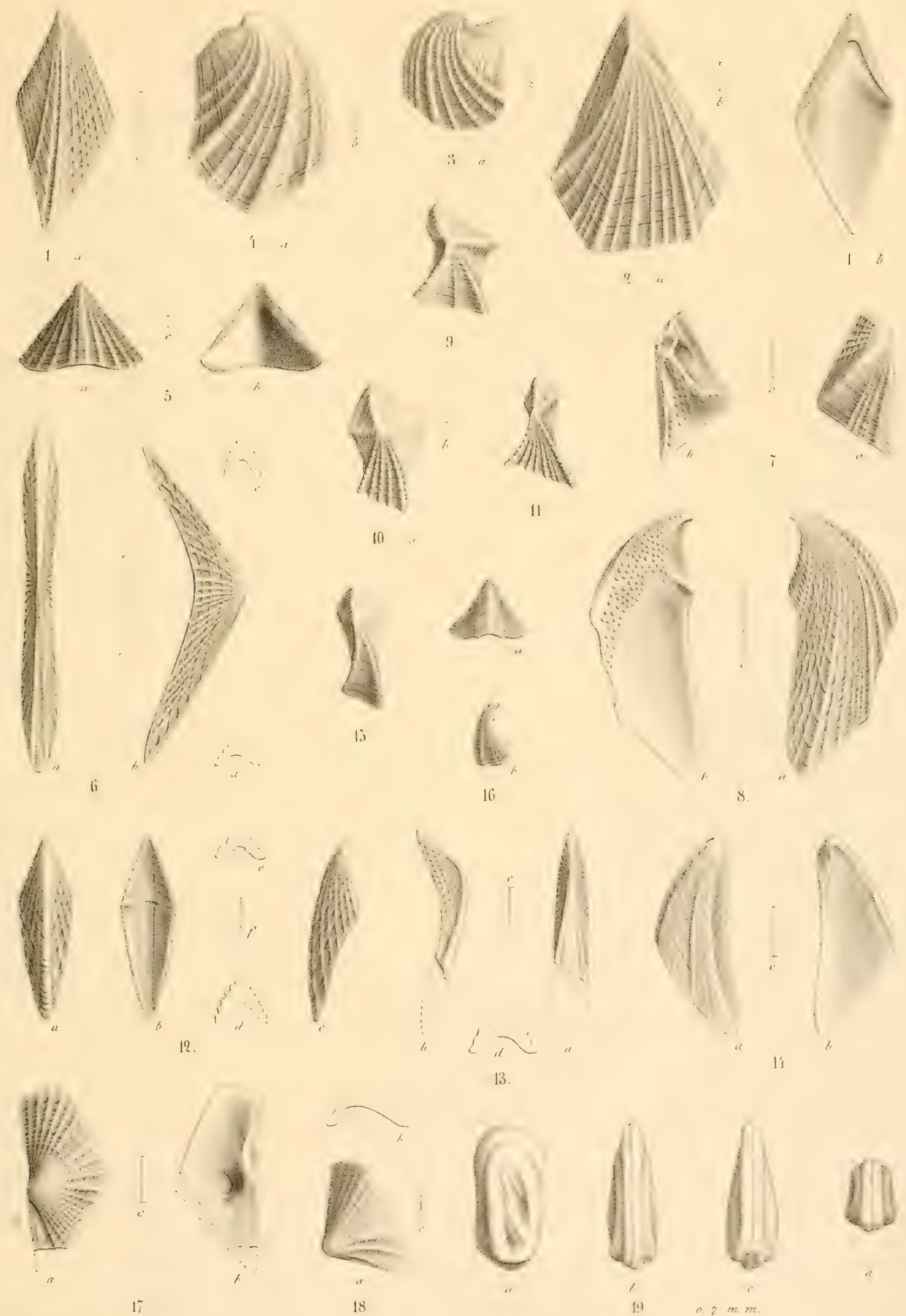




\section{PLANCHE V.}

Figure 1. Cytherella denticulata BosQuet. Page 51.

a. Valve gauche, recueillie dans le Système Maestrichtien de Gronsveld, vue en dessus. De ma collection.

b. Carapace entière, provenant du même terrain de Bemelen, vue du côté supérieur. De ma collection.

c. La même, vue du côté inférieur.

d. La même, vue par l'extrémité antérieure.

Figure 2. Cytherella Williamsonia Jowss. Page 52.

a. Valve gauche, provenant du Système Sénonien de Pesaken, vue en dessus. De ma collection.

b. Carapace entière, de la même couche de Slenaken, vue du côté dorsal. De ma collection.

c. La même, vue du côté pectoral.

d. La même, vue par l'extrémité antérieure.

Figure 3. Bairdia arcuata Von Münster spec. Page 59.

a. Valve gauche, du calcaire grossier à silex gris de St. Pierre, vie en dessus. De ma collection.

b. Carapace entière, de la même localité, vue du côté dorsal. De ma collection.

c. La même, vue du côté pectoral.

d. La même, vue par l'extrémité antérieure.

Figure 4. Bairdia arcuata, Var. $B$. gracilis Boseuet. Page 60.

a. Valve gauche, provenant du Système Maestrichtien de Kunraede, vue en dessus. De ma collection.

b. Carapace entière, provenant de la même localité, vue en dessus. De ma collection.

c. La même, rue en dessous.

đ. La même, vue par l'extrémité antérieure.

Pigure 5. Cytheridea Harrisiana Joves spec, Page 63.

$a$. Carapace, montrant la valve gauche, vue en dessus. De ma collection.

6. La même, vue du côté dorsal.

c. La même, vue du côté pectoral.

d. La même, vue par l'extrémité antérieure.

Figure 6. Cytheridea ovata Boseuet. Page 63.

a. Valve gauche, provenant du terrain Maestrichtien de Fauquemont, vue en dessus. De ma collection.

b. Carapace entière, de la même localité, vue du côté dorsal. De ma collection.

c. La même, vue du côté pectoral.

d. La même, vue par l'extrémité antérieure.
Figure 7. Cythere euglypha Bosquet. Page 74.

a. Valve gauche, provenant du Système Maestrichtien de Nedercanne, vue en dessus. De ma collection.

b. Carapace entière, provenant de la même localité, vue du côté dorsal. De ma collection.

c. La même, vue du côté pectoral.

d. La même, vue par l'extrémité antérieure.

Figure 8. Cythere striato-costata Bosquex. Page 77.

a. Valve gauche, du Système Maestrichtien de Bemelen, vue en dessus. De ma collection.

b. Carapace entière, provenant de la même localité, vue du côté dorsal. De ma collection.

c. La même, vue du côté pectoral.

d. La même, vue par l'extrómité antérieure.

Figure 9. Cythere propinqua Bosever. Page. 78.

a. Valve gauche, provenant du Système Maestrichtien de Bemelen, vue en dessus. De ma collection.

๖. Carapace entière, recueillie dans la même localité, vue du côté supérieur. De ma collection.

c. La même, rue du côté inférieur.

d. La même, vue par l'extrémité antérieure.

Figure 10. Cythere radiosa Boseder. Page 79.

a. Valve gauche, de la craie Maestrichtienne de Bemelen, vue en dessus. De ma collection.

b. Carapace entière, provenant du même terrain de Nedercanne, vue du côté dorsal. De ma collection.

c. La même, vue du coté pectoral.

d. La même, rue par l'extrémité antérieure.

Figure 11. Cythere subtetragona Bosauer. Page 80. a. Valve gauche, vue en dessus. De ma collection.

b. Carapace entière, vue du côté supérieur. De ma collection.

c. La même, vue du côté inférieur.

d.La même, vue par l'extrémité antérieure.

Figure 12. Cythere multilamella Bosever. Page 80.

a. Valve gauche, du Système Sénonien de la montagne de St. Pierre, vue en dessus. De ma collection.

b. Carapace entière, provenant de la même localité, vue du côté supérieur. De ma colleetion.

c. La même, vue du côté inférieur.

d. La même, vue par l'extrémité antérieure. 

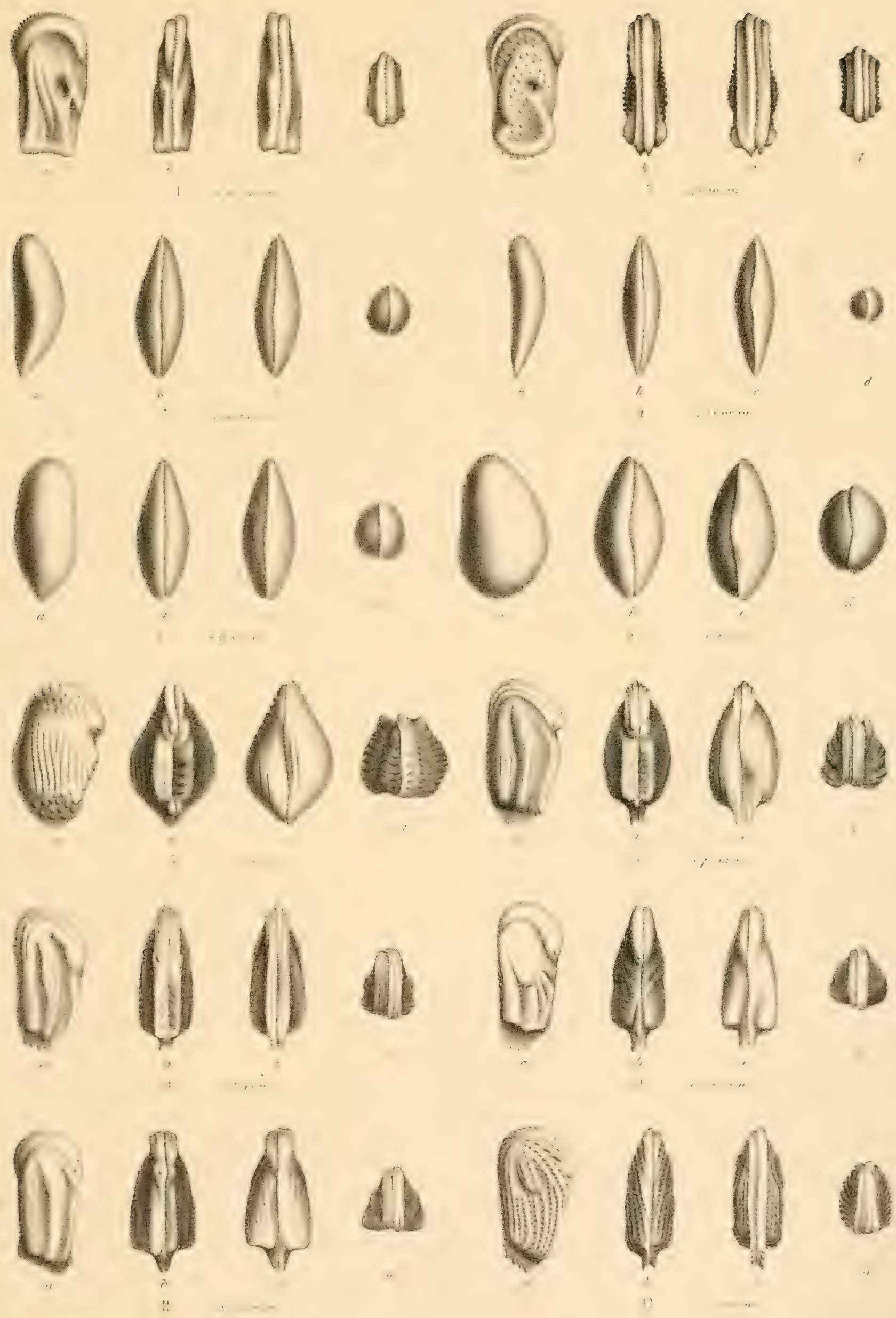




\section{PLANCHE VI.}

Figure 1. Cythere puncturata Bosquet. Page 81.

a. Valve gauche, provenant du Système Maestrichtien de Geulhem, vue en dessus. De ma collection.

๖. Carapace entière, provenant de la même localité, vue du côté dorsal. De ma collection.

c. La même, vue du côté pectoral.

d. La même, vue par l'extrérnité antérieure.

Figure 2. Cythere vesiculosa Bosquet. Page 82.

a. Valve gauche, du terrain Maestrichtien de Nedercanne, vue en dessus. De ma collection.

z. Carapace entic̀re, prosenant du même terrain de Gronsveld, vue da côté dorsal. De ma collection.

c. La même, vue du côté pectoral.

đ. La même, vue par l'extrémité antérieure.

Figure 3. Cythere cerebralis Bosquet. Page 83.

a. Mon échantillon provenant de Bemelen, montrant la valve gauche en dessus.

Ђ. Ie même échantillon, vi du côté supérieur.

c. Le même, vu du côté inférieur.

đ. Le même, vo par l'extrémité antérieure.

Figure 4. Cythere gibberula Bosever. Page 84.

a. Valve gauche, du Systc̀me Maestrichtien de Bemelen, vue en dessus.

๖. Carapace entière, provenant de la même localité, vue du côté du dos. De ma collection.

c. La même, vue du côté pectoral.

đ. La même, vue par l'extrémité antérieure.

Figure 5. Cythere strangulata BosQuet. Page 84.

a. Carapace entière montrant la valve gauche en dessus.

De ma collection.

b. La même, vue du côté supérieur.

c. La même, vue du côté inférieur.

d. La même, vue par l'extrémité antéricure.

Figure 6. Cythere umbonella Bosquet. l'age S5.

a. Valve gauche, du terrain Maestrichtien de Bemelen, vue en dessus. De ma collection.

๖. Carapace entière, provenant de la même localité, vue du côté dorsal. De ma collection.

c. La même, vue du côté pectoral.

d. La même, vue par l'extrémité antérieure.
Figure 7. Cythere longispina Boseuer. Page 86.

a. Valve gauche, provenant du Syst. Sénonien de Slenaken, vue en dessus. De ma collection.

b. Carapace entière, provenant de la même localité, vute du côté supérieur. De ma collection.

c. La même, vue du côté inférieur.

d. La même, vue par l'extrémité antérieure.

Figure 8. Cythere sagittata Bosquer. Page 87.

a. Carapace entière, montrant la valve gauche en dessus, et provenant du terrain Maestrichtien de Bemelen. De ma collection.

b. La même, vue du côté dorsal.

c. La même, vue du côté pectoral.

d. La même, vue par l'extrémité antérieure.

Figure 9. Cythere orchidea Bosquer. Page 88.

a. Valve gauche, du terrain Maestrichtien de Nedercanne, vue en dessus. De ina collection.

b. Carapace entière, du Système Maestrichtien de Fauquemont, vue du côté supérieur. De ma collection.

c. La même, vue du côté inférieur.

d. La même, vue par l'extrémité antérieure.

Figure 10. Cythere complanata Bosquer. Page 89. $a$. Valve gauche, vue en dessus. De ma collection.

$b$. Carapace entière, vue du côté dorsal. De ma collection. c. La même, vue du côté pectoral.

d. La même, vue par l'extrémité antérieure.

Figure 11. Cythere lepida Bosquet. Page 89.

a. Valve gauche provenant du Système Maestrichtien de St. Pierre, vue en dessus. De ma collection.

b. Carapace entière, du même Système de Bemelen, vue du côté supérieur. De ma collection.

c. La même, vue du côté inférieur.

d. La même, vue par l'extrémité antérieure.

Figure 12. Cythere quadridentata Boseuet. Page 90.

a. Valve gauche, du terrain Maestrichtien de Nedercanne, vue en dessus. De ma collection.

6. Carapace entière, provenant de la même localité, vue du côté supérieur. De ma collection.

c. La même, vue du côté inférieur.

d. La même, vue par l'extrémité antérieure. 

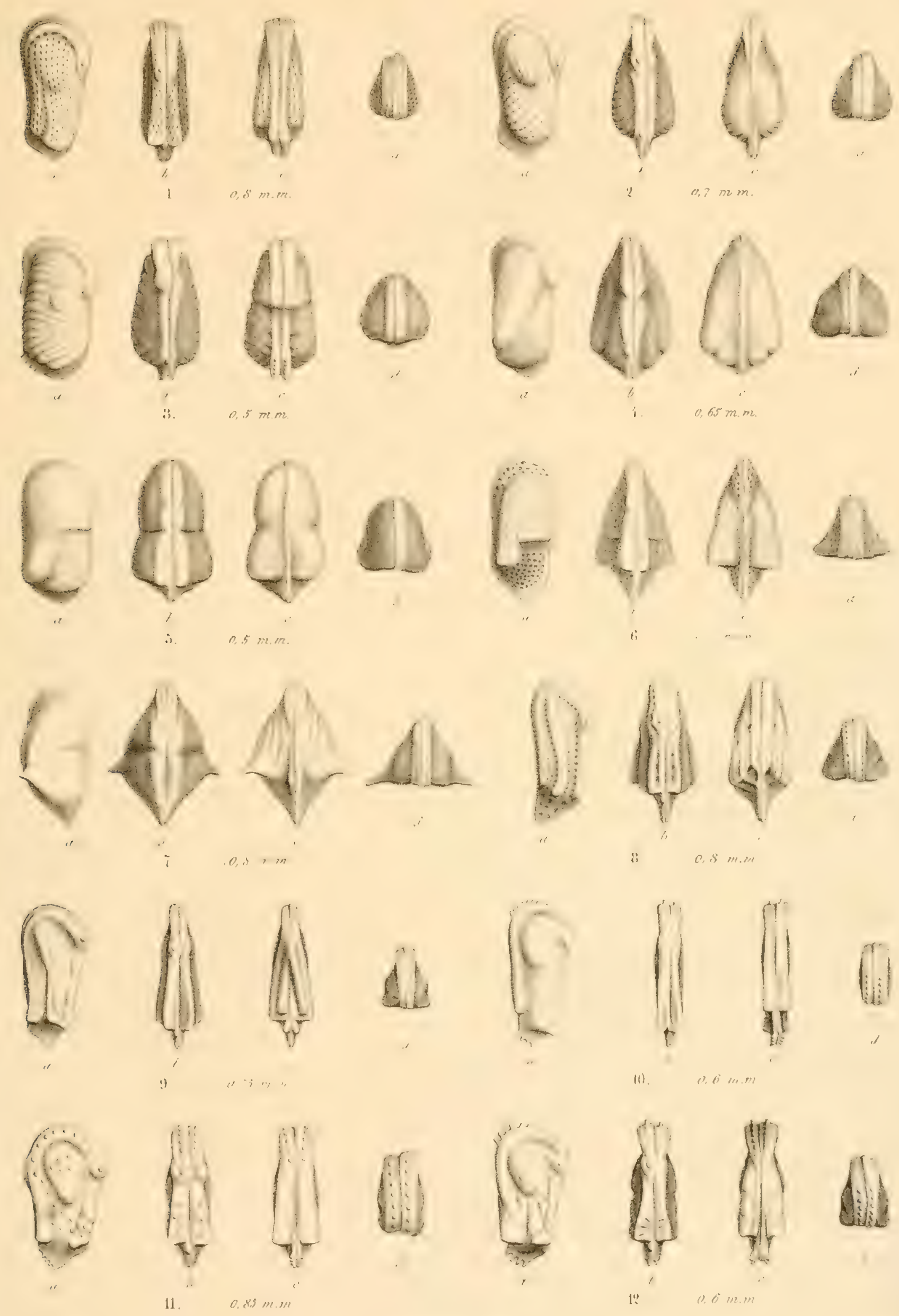




\section{PLANCHE VII.}

Figure 1. Cythere arenosa Bosquer. Page 91.

$\alpha$. Valve gauche, provenant du Système Maestrichtien de Gronsveld, vue en dessus. De ma collection.

b. Carapace entière, de la même localité, vue du côté supérieur, de ma collection.

c. La même, vue du côté inférieur.

d. La même, vue par l'extrémité antérieure.

Figure 2. Cythere variolata Bosever. Page 91,

a. Valve gauche, du Système Maestrichtien de St. Pierre, vue en dessus. De ma collection.

b. Carapace entière, provenant de la même localité, vue du côté dorsal. De ma collection.

c. La même, vu du côté pectoral.

d. La même, vue par l'extrémité antérieure.

Figure 3. Cythere labyrinthica Boseuer. Page 93. a. Valve gauche, provenant du Système Maestrichtien de St. Pierre, rue en dessus. De ma collection.

Ђ. Carapace entière, de la même localité, vue du côté supérieur.

c. La même, vue du côté inférieur.

đ. La même, vue par l'extrémité antérieure.

Figure 4. Cythere elegantula Boseuet. Page 94.

a. Carapace entière, montrant la valve gauche en dessus. De ma collection.

7. La même, vue du côté supérieur.

c. La ınême, vue du côté inférieur.

d. La même, vue par l'extrémité antérieure.

Figure 5. Cythere horridula Bosquet. Page 95.

$a$. Valve gauche, du terrain Maestrichtien de Bemelen, vue en dessus. De ma collection.

b. Carapace entière, provenant de la même localité, vue du côté dorsal.

c. La même, vue du côté pectoral.

d. La même, rue par l'extrémité antérieưre.

Figure 6. Cythere eximia Bosquet. Page 96.

a. Valve gauche, provenant du Système Maestrichtien de St. Pierre, vue en dessus. De ma collection.

8. Carapace entière, provenant de la même localité, vie du côté supérieur. De ma collection.

c. La même, vue du côté inférieur.

¿. La même, vue par l'extrémité antérieure.
Figure 7. Cythere ornatissima Reuss, spec.

Var. A. nodulosa Boseuet. Page 98.

a. Valve gauche, provenant du terrain Maestrichtien de Nedercanne, rue en dessus. De ma collection.

b. Carapace entière, de la même localité, vue du côtédorsal. De ma collection.

c. La même, vue du côté pectoral.

d. La même, vue par l'extrémité antérieure.

Figure 8. Cythere celleporacea Bosquet. Page 101.

$a$. Valve gauche, provenant du système Maestrichtien de Gronsveld, vue en dessus. De ma collection.

b. Carapace entière, recueillie dans la même localité, vue du côté dorsal. De ma collection.

c. La même, vue du côté pectoral.

d. La même, vue par l'extrémité antérieure.

Figure 9. Cythere semicancellata Bosouer. Page 102.

a. Valre gauche, provenant du Système Sénonien e St. Pierre, vie en dessus. De ma collection.

b. Carapace entière, du même Système de Bemelen, vue du côté dorsal. De ma collection.

c. La même, vu du côté pectoral.

d. La même, vue par l'extrémité antérieure.

Figure 10. Cythere phylloptera Boseder. Prge 106.

a. Valve gauche, provenant du Système Sénonieu de St. Pierre, vue en dessus. De ma collection.

๖. Carapace entière, du même Système crétacé de Ciply, en Belgique, vue du côté dorsal. De ma collection.

c. La même, vue du côté pectoral.

d. La même, vue par l'extrémité antérieure.

Figure 11. Cythere laticristata Boseuer. Page 108.

$a$. Valve gauche, provenant du Syst. Sénonien de Slenaken, vue en dessus. De ma collection.

๖. Carapace entière, provenant de la même localité, vue du côté supérieur. De ma collection.

c. La même, vue du côté inférieur.

d. La même, vue par l'extrémité antérieure.

Figure 12. Cythere trigonoptera Bosquet. Page 109.

a. Valve gauche, provenant du Système Maestrichtien de Geulhem, vue en dessus. De ma collection.

b. Carapace entière, du même Système de Bemelen, vue du côté superieur. De ma collection.

c. La même, vue du côté inférieur.

d. La même, vue par l'extrémité antérieure. 

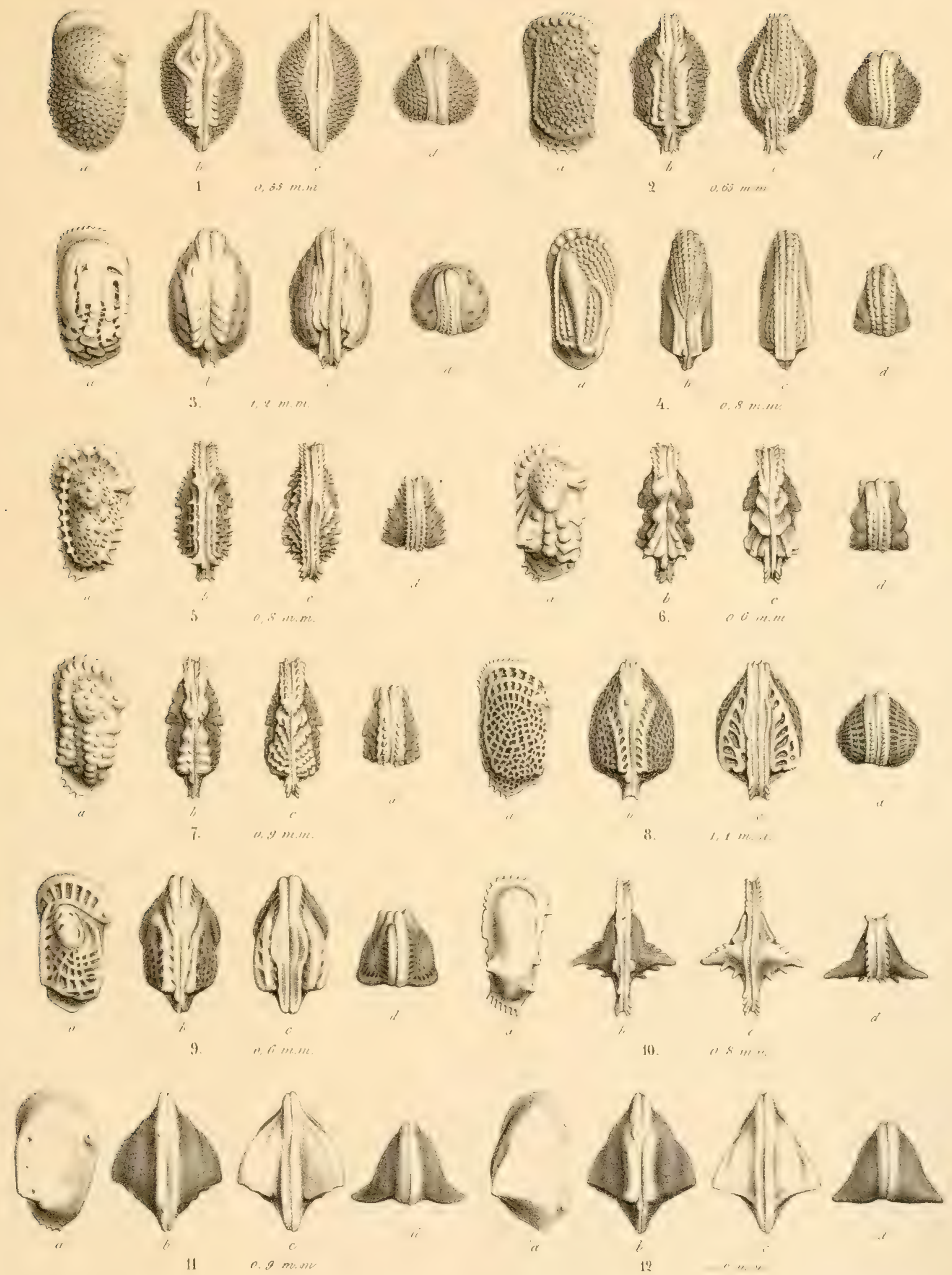




\section{PLANCHE VIII.}

Wigure 1. Cytherella ovata Roemer, spec. Page 45.

a. Valve gauche, provenant du Système Maestrichtien de St. Pierre, vue en dessus. De ma collection.

b. Valve droite, vue du côté interne.

c. Valve gauche, vue du même côté.

d. Carapace entière, receuillie dans le terrain Maestrichtien de St. Pierre, vue du côté dorsal. De ma collection.

e. La même, vue du côté pectoral.

f. La même, vue par l'extrémité antérieure.

Figure 2. Cytherella Munsteri Roemer, spec. Page 48.

a. Valve gauche, provenant du terrain Maestrichtien de Bemelen, vue en dessus. De ma collection.

४. Carapace entière, provenant de la même localité, vue du côté supérieur. De ma collection.

c. La même, vue du côté inférieur.

d. La même, vue par l'extrémité antérieure.

Higure 3. Bairdia subglobosa ${ }^{1}$ ) Bosquet. Page 55.

$a$. Valve gauche, provenant du terrain Maestrichtien de l'auquemont, vue en dessus. De ma collection.

h. Carapace entière, receuillie dans le même terrain, et vue du côté supérieur. De ma collection.

c. La même, vue du côté inférieur

đ. La même, vue par l'extrémité antérieure.

Figure t. Bairdia subdeltoïdea Von Münster, spec. Page 5.

a. Valve gauche, receuillie dans le calcaire grossier à silex gris, à St. Pierze, vue en dessus. De ma collection.

b. Valve droite, vue du côté interne.

c. Valve gauche, vue du même côté.

d. Carapace entière, provenant du Système Maestrichtien de Nedercanne, vue du côté supérieur. De ma collection.

e. La même, vue du côté inférieur.

f. La même, vue par l'extrémité antérieure.

Figure 5 Cytheridea Jonesiana 'Bosevet. Page 64.

a. Valve gauche, provenant du Système Hervien? de Benzenrathhof, et vue en dessus. De ma collection.

b. Carapace entière, provenant du même terrain et vue $d u$ coté dorsal. De ma collection.

c. La même, vue du côté pectoral.

d. La même, vue par l'extrémité antérieure.
Figure 6. Cythere fusiformis Bosquer. Page 69.

a. Valve gauche, receuillie dans le terrain Maestrichtien de Geulhern, vue en dessus. De ma collection.

b. Carapace entière, provenant de la même localité et vue du côté dorsal. De ma collection.

c. La même, vue du côté pectoral.

d. La même, vue par l'extrémité antérieure.

Figure \%. Cythere Favrodiana Boseoet. Page 70.

$a$. Valve gauche, receuillie dans le Système Maestrichtien de Fauquemont, vue en dessus. De ma collection.

6. Carapace entière, receuillie dans la même localité et vue du côté supérieur. De ma collection.

c. La même, vue du côté inférieur.

d. La méme, vue par l'extrémité antérieure.

Figure 8. Cythere concentrica Reuss spec. Page 71.

a. Valve gauche, provenant du calcaire grossier à silex gris de la montagne de St. Pierre et vue en dessus. De ma collection.

b. Carapace entière, vue du côté dorsal. De ma collection.

c. La même, vue du côté pectoral.

d. La mêne, vue par l'extrémité antérieure.

Figure $\mathrm{S}^{\prime}$. Cythere concentrica.

Var. B. Subcarinata Bosquer. Page 71.

a. Valve gauche, provenant du terrain Maestrichtien de Gronsveld, vue en dessus. De ma collection.

b. Carapace entière, vue du côté supérieur. De ma collection.

c. La même, vue du côté inférieur.

d. La même, vue par l'extrémité antérieure.

Figure 9. Cythere furcifera Bosevet. Page 73.

a. Valve gauche, du Systìme Maestrichtien de Gronsveld, vue en dessus. De ma collection.

๖. Carapace entière, vue du côté dorsal. De ma collection.

c. La même, vue du côté pectoral.

c. La même, vue par l'extrémité antérieure.

Figure 10. Cythere interrupta Bosquet. Page 7t.

a. Valve gauche, receuillie dans le Système Maestrichtien de St.Pierre, vue en dessus. De ma collection.

b. Carapace entière, provenant de la même localité et vue du côté dorsal. De ma collection.

c. La même, vue du côté pectoral.

d. La même, vue par l'extrémité antérieure.

1) Après que l'impression des premières fenilles de ce mémoire fût terminée, je me suis aperçu, par la découverte d’individus interméliaires, que les échantillons décrits sous le nom de Bairdia subglobosa ne sont que des jeunes individus de la B. subdeltoïdea. 


$$
\begin{aligned}
& 00000000 \\
& 00000000 \\
& 00000000 \\
& 10001000 \\
& 10000004 \\
& 00400000
\end{aligned}
$$






\section{PLANCHE 1X.}

Figure 1. Cythere pulchella Boseover. Page 76.

a. Valve gauche provenant du calcaire grossier à silex gris entre St. Pierre et Petit-Lanaye, vue en dessus. De ma collection.

6. Carapace entière, provenant de la même localité, vue du côté supérieur. De ma collection.

c. La même, vue du côté inférieur.

d. La inême, vue par l'extrémité antérieure.

Figure 2. Cythere pulchella, Var. B. Bosquet. Pag. 76.

a. Valve gauche, provenant du Système Maestrichtien de Gronsveld, vue en dessus. De ma collection.

8. Carapace entière, provenant de la même localité, vue du côté supérieur. De ma collection.

c. La même, vue du côté inférieur.

d. La même, vue par l'extrémité antérieure.

Figure 3. Cythere elegans Bośuet. Page 78.

$a$. Valve gauche, provenant du Système Maestrichtien de Bemelen, vue en dessus. De ma collection.

も. Carapace entière, receuillie dans la même localité et vue du côté supérieur. De ma collection.

c. La même, vue du côté inférieur.

d. La même, vue par l'extrémité antérieure.

Figure 4. Cythere macropthalma Boseuer. Page 86 .

$a$. Valve ganche, du calcaire grossier à silex gris de la la montagne de St. Pierre. De ma collection.

b. Carapace entière, provenant de la même localité, vue du côté dorsal. De ma collection.

$c$. La même, vue du côté pectoral.

đ. La même, vue par l'extrémité antérieure.

Figure 5. Cythere hieroglyphica Bosquet. Page 92. a. Valve gauche, provenant du terrain Maestrichtien de St. Pierre, vue en dessus. De ma collection.

b. Carapace entière, recueillie dans la même localité, vue du côté dorsal. De ma collection.

c. La même, vuè dư côté pectoral.

d. La même, vue par l'extrémité antérieure.

Figure 6. Cythere ornatissima heuss, spec. Page 97.

a. Valve gauche, provenant du psammite glauconifère de Pesaken vue en dessus. De ma collection.

b. Carapace entiére, provenant de la même localité, vue du côté supérieur. De ma collection.

c. La même, vue du côté inférieur.

d. La même, vue par l'extrémité antérieure.
Figure 7. Cythere Koninckiana Bosquet. Page 100.

$a$. Valve gauche, provenant du Système Maestrichtien de Nedercanne, vue en dessus. De ma collection.

b. Carapace entière, du même terrain de Fauquemont, vue du côté supérieur. De ma collection.

c. La même, vue du côté inférieur.

d. La même, vue par l'extrémité antérieure.

Figure 8. Cythere ornata Bosauet. Page 103.

a. Valve gauche, provenant du terrain Maestrichtien de St. Pierre, vae en dessus. De ma collection.

b. Carapace entière, receuillie dans la même terrain, vue du côté supérieur. De ma collection.

c. La même, rue du côté inférieur.

d. La même, vue par l'extrémité antérieure.

Figure 9. Cythere serrulata Bosever. Page 104.

a. Valve gauche, du calcaire ì silex gris de St. Pierre, vue en dessus. De ma collection.

b. Carapace entière, provenant de la même localité, vue du côté supérieur. De ma collection.

c. La même, vue du côté inférieur.

d. La même, vue par l'extrémité antérieure.

Figure 10. Cythere alata Bosquet. Page 107.

$a$. Valve gauche, provenant du Système Maestrichtien entre St. Pierre et Petit-Lanaye, vue en dessus. De ma collection.

๖. Carapace entière, de la même localité, vue du côté dorsal. De raa collection.

c. La même, vue du côté pectoral.

d. La même, vue par l'extrémité antérieure.

Figure 11. Cyprella ovulata Bosevet. page 114.

a. Valve gauche, provenant du calcaire grossier ì silex gris, entre St. Pierre et Petit-Lanaye, vue en dessus. De ma collection.

b. Carapace entière, provenant de la même localité, vue du côté supérieur. De ma collection. c. La même, vue du côté inférieur.

Figure 12. Cyprella Koninckiana Bosquet. Page 115.

$a$. Valve gauche, receuillic dans le terrain Maestrichtien de Bemelen, vue en dessus. De ma collection.

b. Valve droite, vue en dedans.

c. Valve gauche, vue du même côté. 


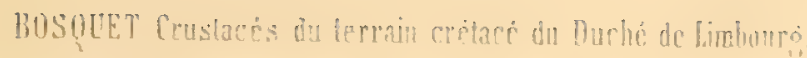
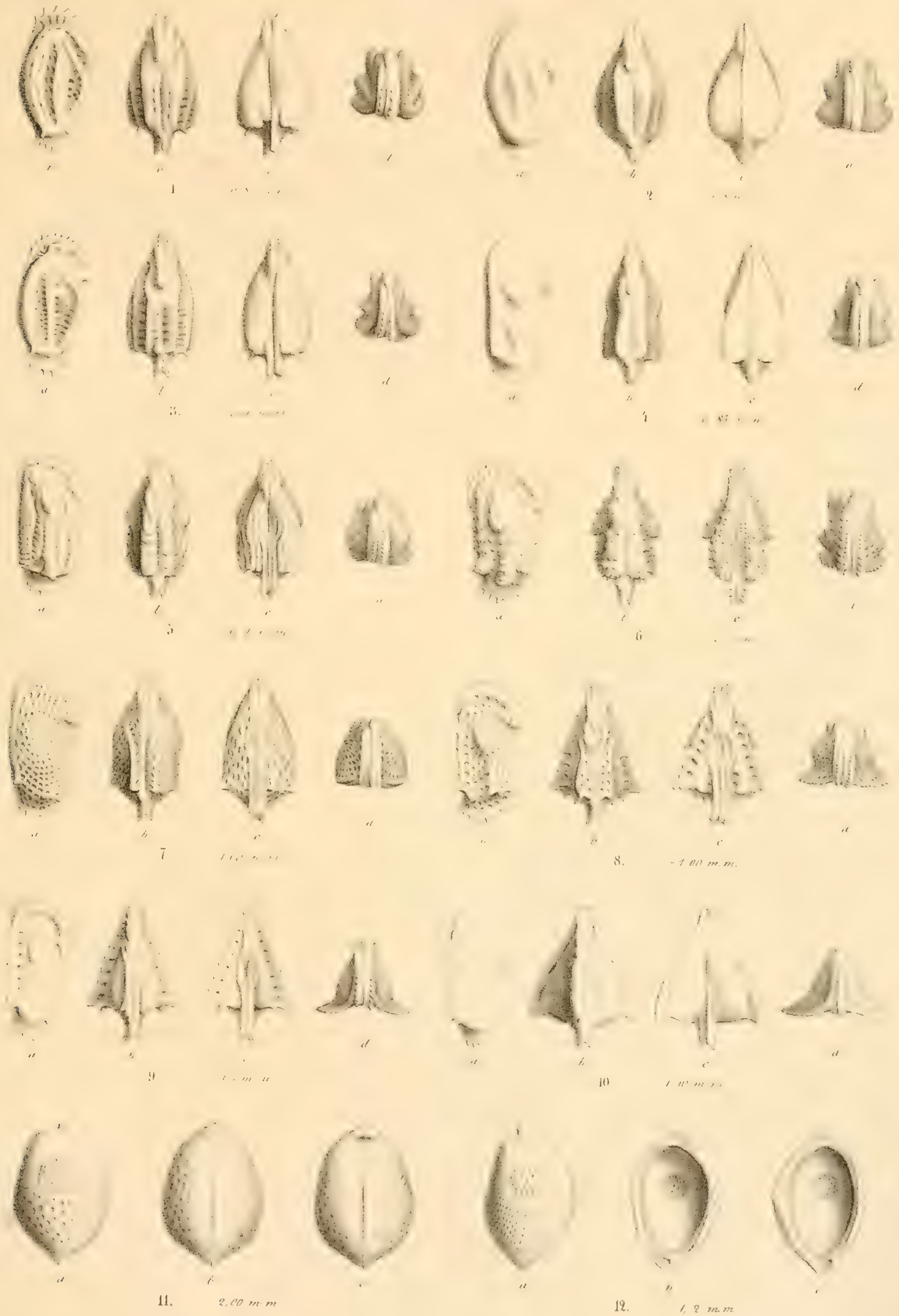




\section{PLANCHE $X$}

Figure 1. Cythere minuta Bosquet. Page 109.

a. Valve gauche, provenant da Système Maestrichtien de Nedercanne, vue en dessus. De ma collection.

b. Carapace entière, provenant du même Système de Bemelen, rue du côté dorsal. De ma collection.

c. La même, vue du côté pectoral.

d. La même, vue par l'extrémité antérieure.

Figure 2. Cythere Hagenowi Boseuer. Page 110.

a. Valve gauche, provenant du Système Maestrichtien de Gronsveld, vue en dessus. De ma collection.

b. Carapace entière, recueillie dans le même localité, vue dù côt: dorsal. De ma collection.

c. La même, vue du côté pectoral.

d. La même, vue par l'extrémité antérieure.

Figure 3. Cythere macroptera Bosquet. Page 111.

a. Valve gauche, provenant du Système Maestrichtien de Bemelen, rue en dessus. De ma collection.

b. Carapace entière, vue du côté supérieur. De ma collection.

c. La même, vue du côté inférieur.

d. La même, vue par l'extrémité antérieure.

Figure 4. Cythere cristata Boseuet. Page 112.

a. Valve gauche, provenant du terrain Maestrichtien de St. Pierre, vue en dessus. De ma collection.

b. Carapace entière, provenant de la même localité, vue du côté dorsal. De ma collection.

c. La même, vue du côté pectoral.

d. La même, vute par l'extrémité antérieure.

Figure 5-8. Oncopareia Bredaï Bosquet. Page 118.

Hig. 5. Céphalothorax (sur lequel les granulations de la surface ont été restaurées d'après celles de plusieurs autres échantillons) de grandeur naturelle, et vu du côté gauche. De ma collection.

"6. Abdomen incomplet, à test parfaitement bien conservé, de grandeur naturelle. De ma collection.

"6'. Echantillon à test conservé, vu du côté gauche et montrant la nageoire caudale un peu mutilée de grandeur taturelle. De la collection du Prés. Dr. VAN BREDA.
Fig. 7. Main droite incomplète, de grandeur naturelle. De ma collection.

" 7', a. Main gauche, apparemment d'un jeune individu, avec une partie du doigt mobile, de grandeur naturelle et vuc du côté externe. De la collection du Prés. Dr. Van Breda.

Ъ. La même main, de grandeur naturelle et vue en dessous.

8. Partie antérieure du céphalothorax d'un jeune individu, avec l'empreinte du rostre, un article de la patte machoire? et avec le support de l'oeil droit, de grandeur naturelle. De ma collection.

Figure 9. Oncoparein? heterodon BosQuet. Page 121.

a. Doigt immobile, provenant de la montagne de St. Pierre, de grandeur naturelle et vue en dessus. De ma collection.

b. Doigt mobile incomplet de la main droite, de grandeur naturelie et vue en dessus. De ma collection.

Figure 10. Mesostylus Faujasii F. Roemer et Brons. pro parte. Page 123.

a. Patte antérieure droite complète, de grandeur naturelle et provenant du Système Maestrichtien de la montagne de St. Pierre. De ma collection.

6. Patte de la seconde paire droite, incomplète et de granclenr naturelle. De ma collection.

Figure 11. Aulncopodia Riemsdyki BosQ. Pnge 125. IIan gauche avec le carpus, provenảnt du Système Maestrichtien de la monlagne de St. Pierre, et vue en dessus. De ma collection.

Figure 12. Stephanometopon granulatum BosedeT. P'age. 127.

a. Carapace incomplète, de grandeur naturelle, vue en dessus. De ma collection.

b. La même, vue par l'extrémité antérieure, pour montrer la forme du front.

c. Main gauche, provenant probablement d'un vieil individu de la même espèce, de grandeur naturelle. De ma collection. 

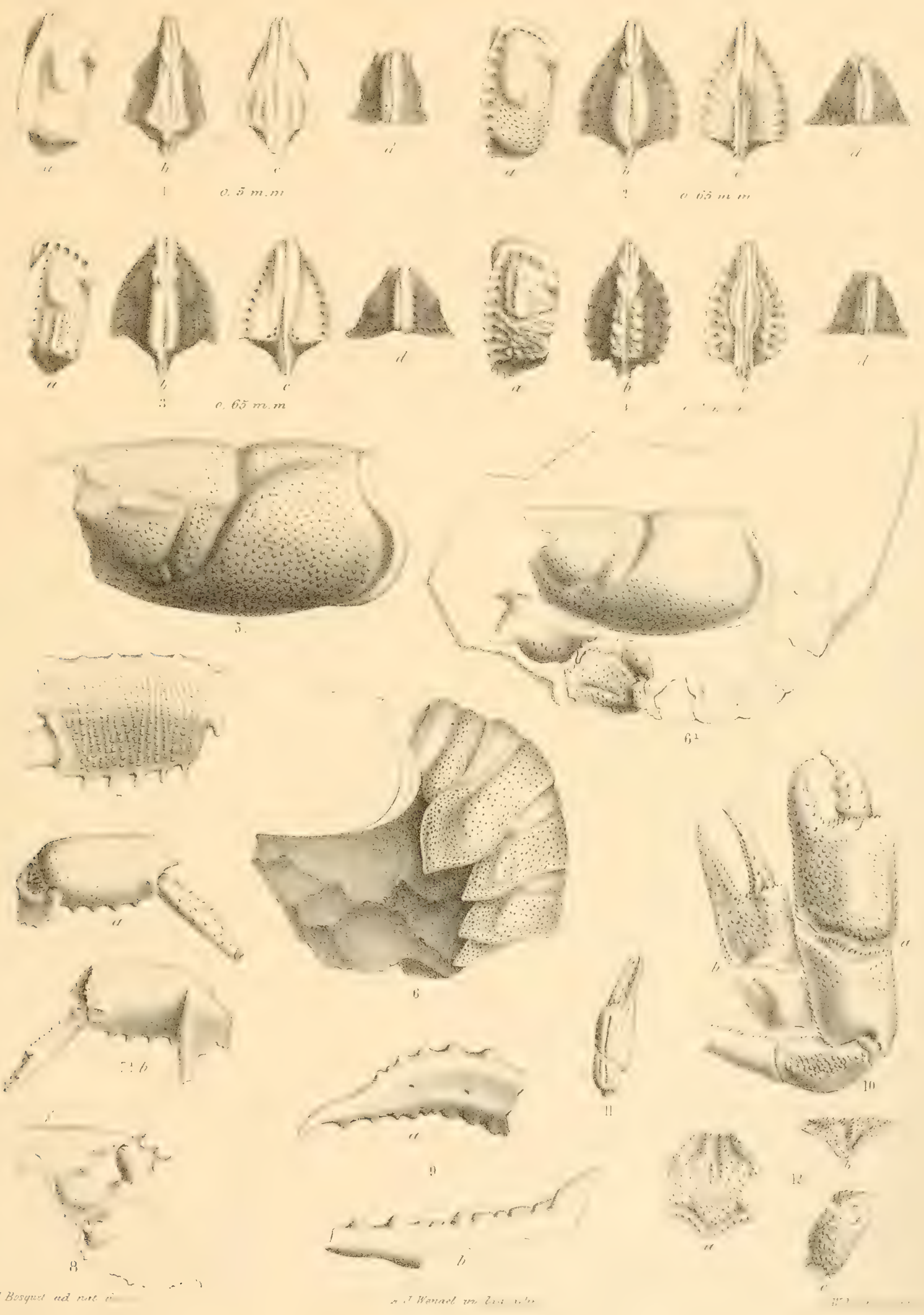
ii 





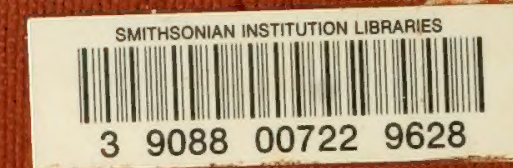

